\title{
DESIGN AND OPTIMIZATION OF A BLOOD VESSEL MIMIC BIOREACTOR SYSTEM FOR THE EVALUATION OF INTRAVASCULAR DEVICES IN SIMPLE AND COMPLEX VESSEL GEOMETRIES
}

\author{
A Thesis \\ Presented to \\ the Faculty of California Polytechnic State University \\ San Luis Obispo \\ In Partial Fulfillment \\ of the Requirements for the Degree \\ Master of Science in Engineering \\ with a Specialization in Biomedical Engineering
}

by

Sara M. Leifer

November 2008 
(C) 2008

Sara M. Leifer

ALL RIGHTS RESERVED 


\section{COMMITTEE MEMBERSHIP}

TITLE:

Design and Optimization of a Blood Vessel Mimic

Bioreactor System for the Evaluation of Intravascular

Devices in Simple and Complex Vessel Geometries
AUTHOR:
Sara M. Leifer
DATE SUBMITTED: $\quad$ November 2008
COMMITTEE CHAIR: $\quad$ Dr. Kristen O'Halloran Cardinal, Assistant Professor

COMMITTEE MEMBER: $\quad$ Dr. Trevor Cardinal, Assistant Professor

COMMITTEE MEMBER: Dr. Lily Laiho, Assistant Professor 


\begin{abstract}
Design and Optimization of a Blood Vessel Mimic Bioreactor System for the Evaluation of Intravascular Devices in Simple and Complex Vessel Geometries

by
\end{abstract}

Sara M. Leifer

Coronary artery disease affects millions of people and the ability to detect and treat the disease is advancing at a rapid rate. As a result, the development of intravascular technologies is the focus of many medical device manufacturers. Specifically, coronary stent implantation is being performed in an increasing number of patients and a number of new stent designs have been introduced to the market, resulting in the need for improved preclinical testing methods. An in vitro tissue engineered "blood vessel mimic" (BVM) system has previously been established and its feasibility for the initial testing of newly emerging intravascular technology has been demonstrated. There are limitations that exist with this original design, however, and the focus of this thesis was to both improve and expand upon the original model. Therefore, research was conducted based on two specific aims. The first aim was to develop a more ideal BVM system to accommodate a wider range of stent lengths and diameters, while allowing for easy graft insertion and seal-ability. The second aim was to develop next generation BVM systems, 
focused on future needs and technology, such as long, angulated and bifurcated geometries.

The work described in this thesis demonstrates that a BVM chamber can be created which has the advantages of easy graft insertion and seal-ability, as well as the ability to accommodate varying sizes of vessel scaffolds, all while maintaining the needs of a tissue engineering bioreactor system. The next generation BVM systems presented demonstrate that the BVM concept can be expanded to meet the needs of long, angulated and bifurcated geometries. Overall, the work in this thesis describes the design and optimization of an in vitro blood vessel mimic bioreactor system for the evaluation of intravascular devices, specifically coronary stents, in simple and complex vessel geometries. 


\section{ACKNOWLEDGEMENTS}

First, I would like to acknowledge and thank the members of my thesis committee: Dr. Kristen O'Halloran Cardinal, Dr. Trevor Cardinal and Dr. Lily Laiho. Thanks for all your guidance and input and for providing assistance and ideas throughout the project.

I would like to acknowledge the students, faculty, and staff of the Biomedical and General Engineering Department at Cal Poly, including Dr. Dan Walsh and Dr. Robert Crockett. Dr. Walsh- Thanks for your constant support and encouragement during my time at Cal Poly. Thank you for believing in me and always encouraging me to reach higher. Dr. Crockett- Thank you for instilling in me the desire to make a difference and for providing me with the skills I needed in the work place.

I would also like to acknowledge the help and support of my fellow graduate students and the 2008 Spring BVM Lab Group: Dalton Chavez, Marc Dawson, Colby James, Dimitri Delagrammaticas, Tiffany Flint, Aubrey Smith and Brian Wong. Dalton- Thanks for madly building biochambers! I couldn't have done it without you. Dimitri- Thanks for using and evaluating the Ideal BVM system. Your input was invaluable to the final design. Colby- Thanks for the awesome SEM images that demonstrate that my system actually works and that cells survive! Aubrey- Thanks for all your help in the lab and for being Dimitri's non-sterile partner. Marc- Thanks for all your input regarding the overall design, especially flow considerations.

To my family- Mom, Eric, Grandma, Grandpa, Debbie, Jonathan, Steven and Dee Dee. Mom- Thanks for being my inspiration. You are the strongest and most courageous woman I know. Without your love and support I would not be who I am today. EricThanks for the adventures and for acknowledging me as Master of the Universe! DebbieThank you for your constant love and support, for listening to my joys and struggles, and for welcoming me with open arms. Jonathan and Steven- Thanks for letting me sleep on the couch and adopting me as your honorary older sister. Grandma and Grandpa- Thanks for loving me so well.

To my Dad- Jeffrey D. Leifer. I love and miss you. I hope I make you proud.

Finally, I would like to thank my thesis advisor, Dr. Kristen O'Halloran Cardinal.

Kristen- Thank you for the opportunity to work on the BVM project and partner in your research. I cannot thank you enough for the time, guidance, support and input you have given me. I look forward to the future of the BVM system. 


\section{DEDICATION}

For my Dad,

Jeffrey D. Leifer

February 29, 1952-July 15, 2001 


\section{TABLE OF CONTENTS}

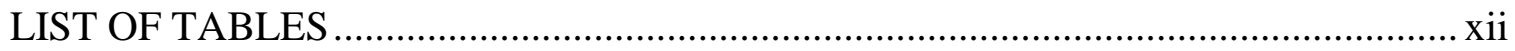

LIST OF FIGURES ................................................................................... xiii

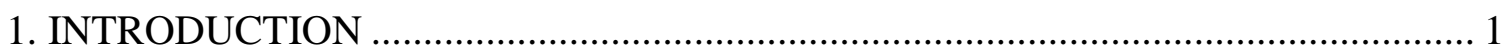

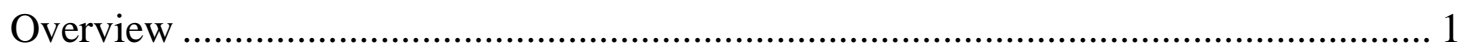

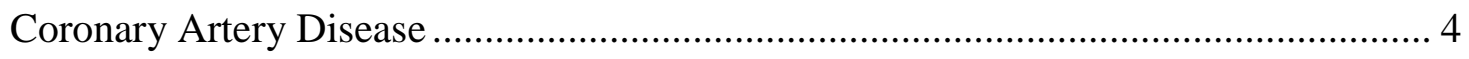

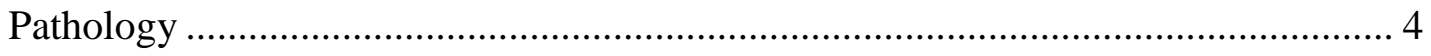

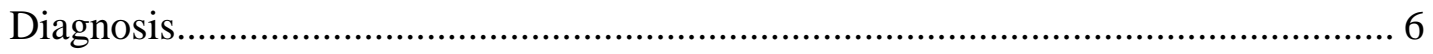

Treatment of Coronary Artery Disease ............................................................... 10

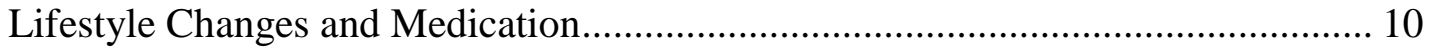

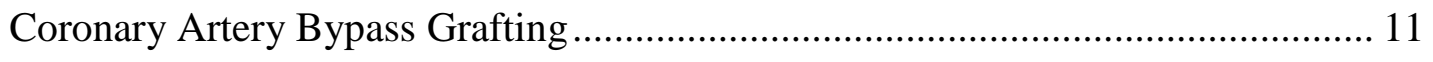

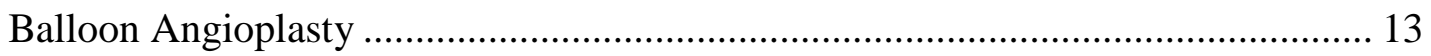

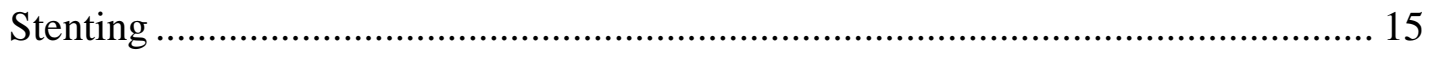

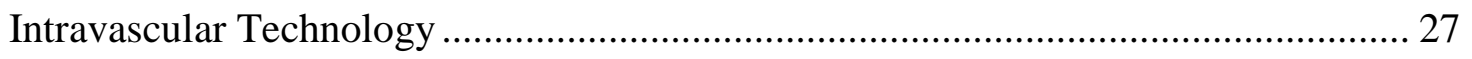

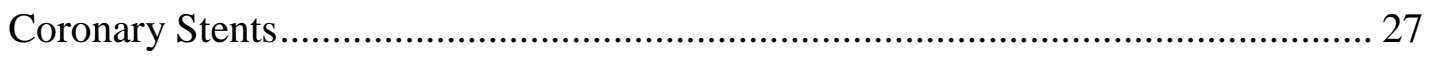

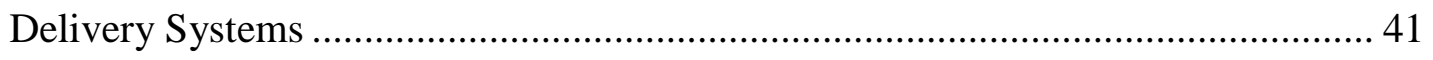

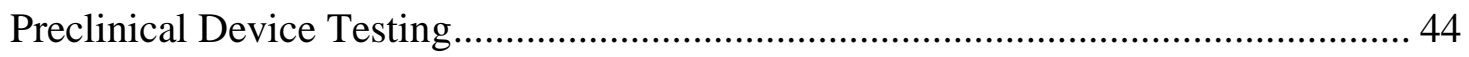

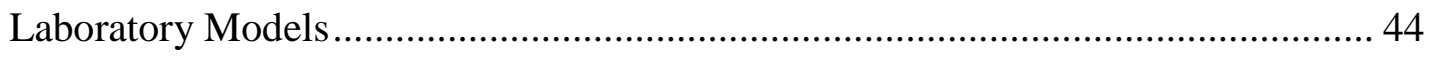

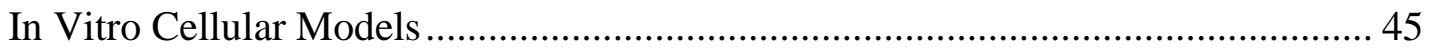

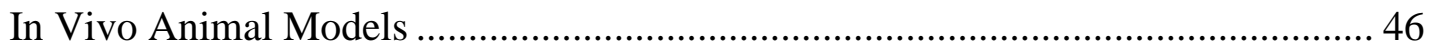

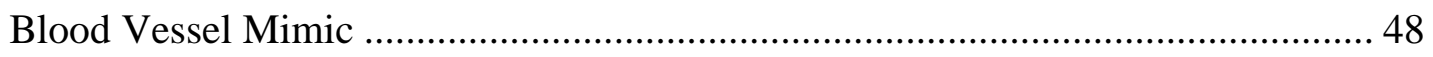

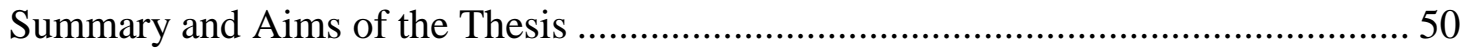


2. INITIAL DESIGN OF AN IDEAL BVM ........................................................ 52

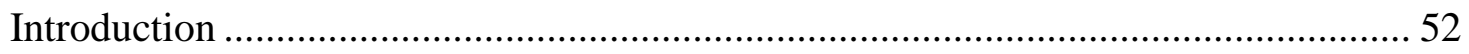

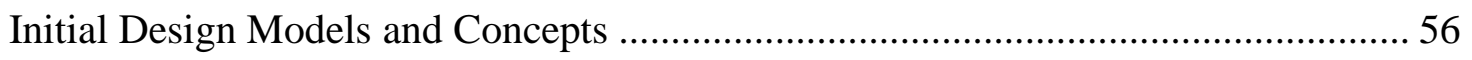

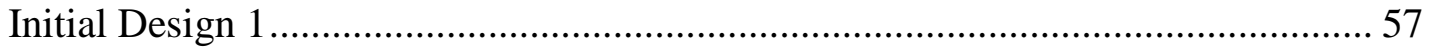

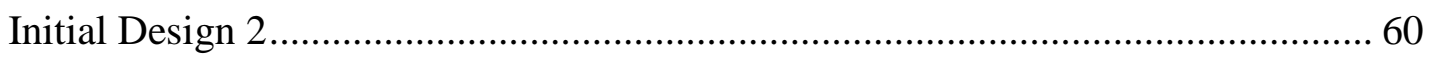

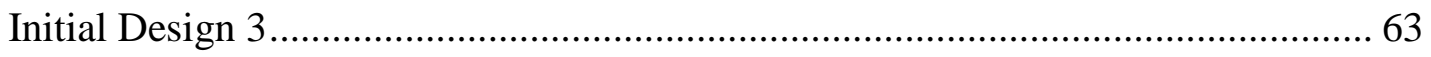

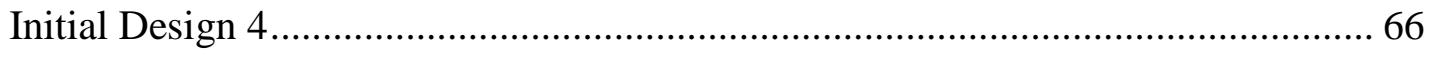

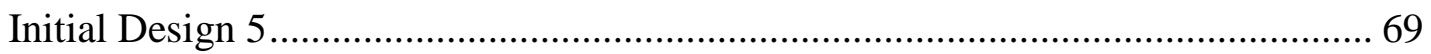

Evaluation of Initial Design Models and Concepts ........................................... 72

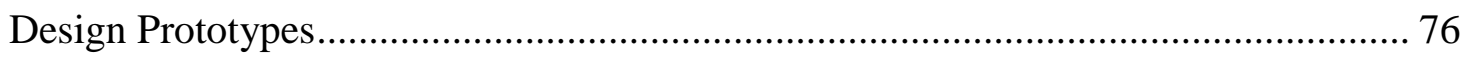

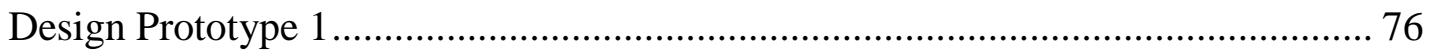

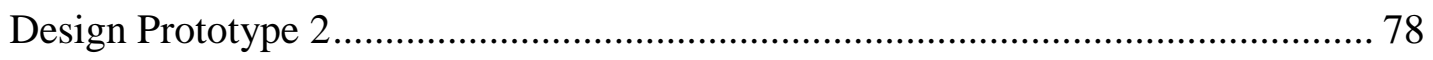

Evaluation of Design Prototypes ............................................................... 79

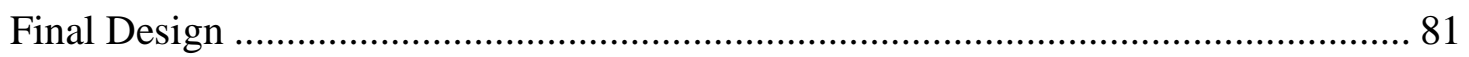

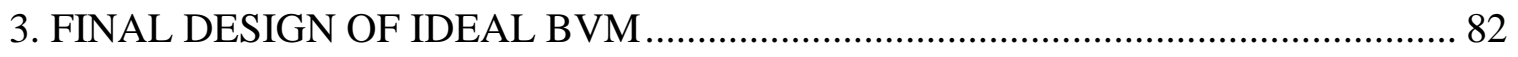

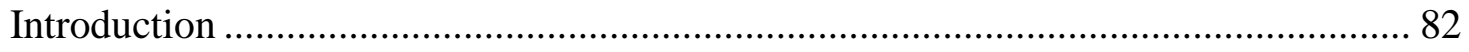

Materials and Methods .................................................................................... 84

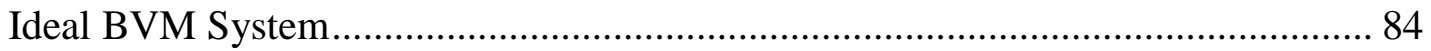

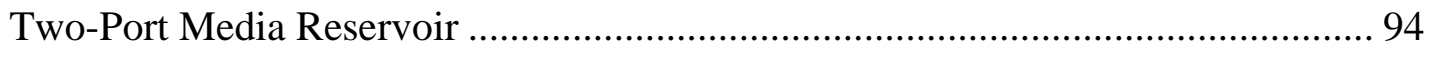

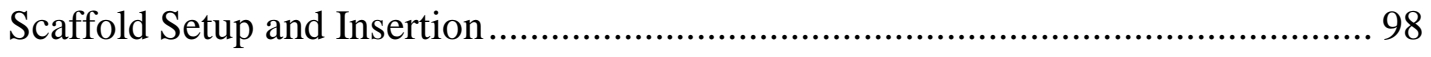

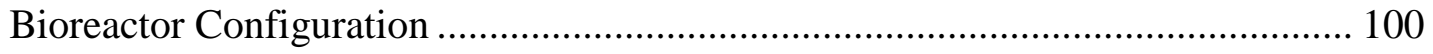

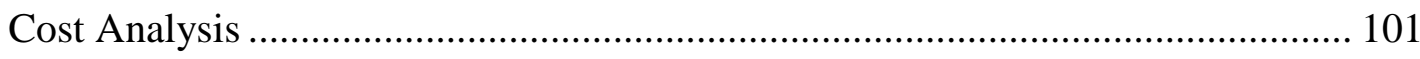

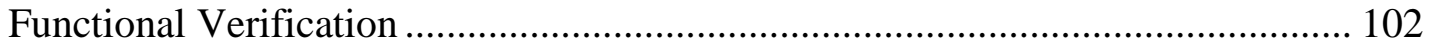


Comparison of Ideal BVM System to Original BVM System ............................ 103

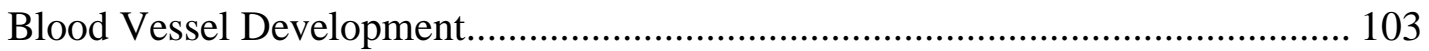

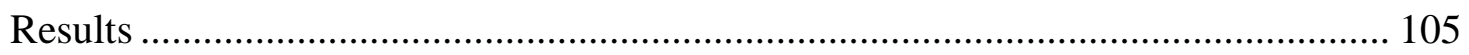

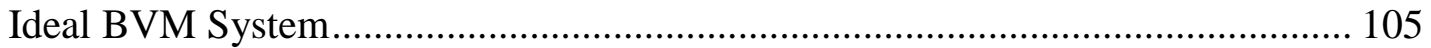

Two-Port Media Reservoir .......................................................................... 107

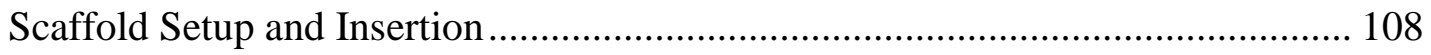

Bioreactor Configuration ....................................................................... 110

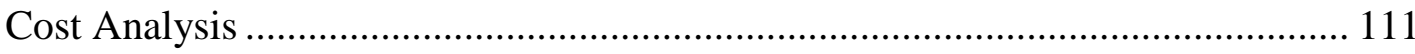

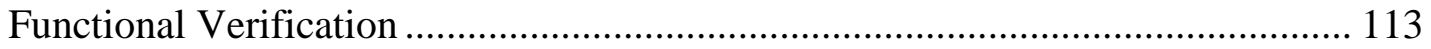

Comparison of Ideal BVM System to Original BVM System ............................ 116

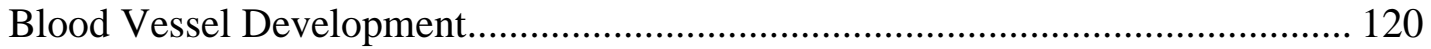

Discussion and Conclusions ........................................................................ 122

4. DESIGN OF NEXT GENERATION BVMs.................................................... 127

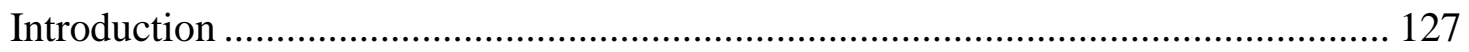

Next Generation BVM System Designs .......................................................... 135

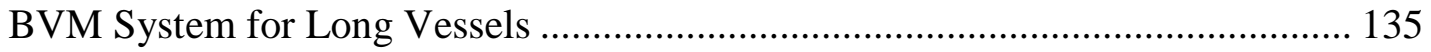

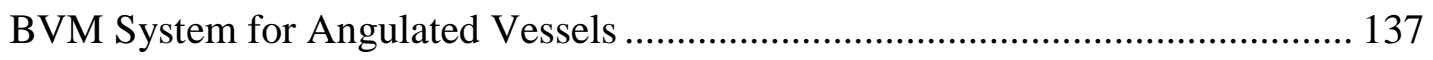

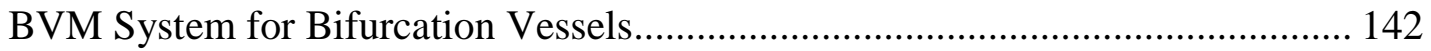

Next Generation BVM System Prototypes ................................................... 149

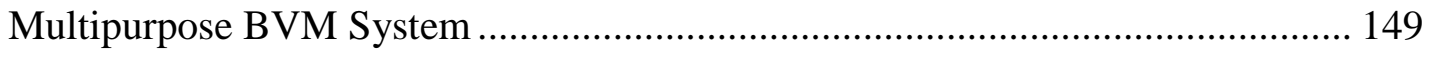

Y-Shaped Bifurcation BVM System ........................................................ 154

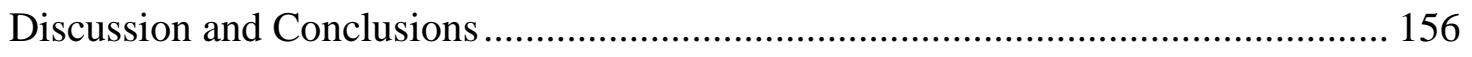




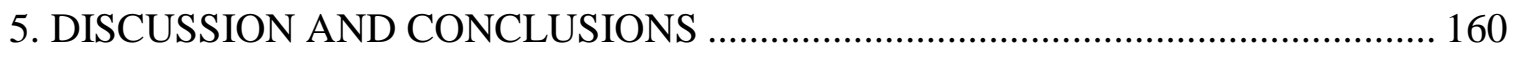

Preclinical Intravascular Device Testing .................................................................. 163

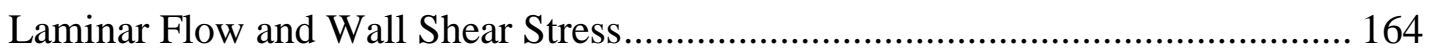

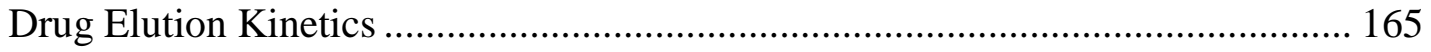

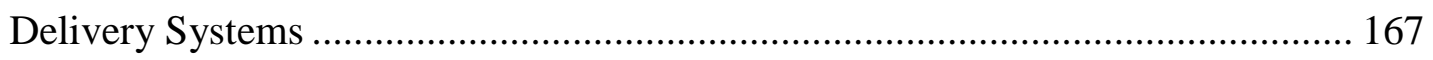

The BVM Concept for Other Tissues.................................................................... 169

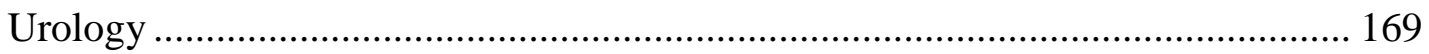

Abdominal Aortic Aneurysm......................................................................... 170

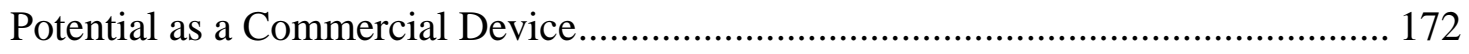

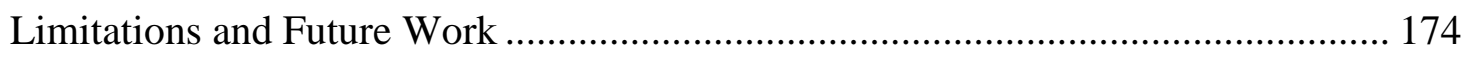

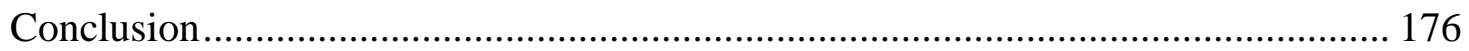

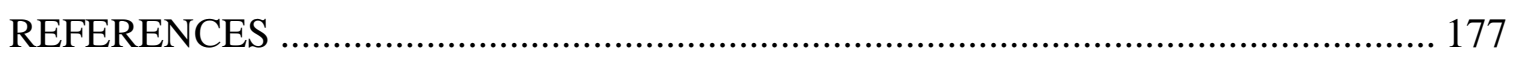

APPENDIX A: LIST OF ABBREVIATIONS ………………................................ 192

APPENDIX B: PART INFORMATION AND DRAWINGS ....................................... 193

APPENDIX C: IDEAL BVM SYSTEM ASSEMBLY ............................................. 212

APPENDIX D: TWO-PORT MEDIA RESERVOIR ASSEMBLY ………………...... 219

APPENDIX E: SCAFFOLD LENGTH VS NUMBER OF COUPLERS USED............ 224

APPENDIX F: BVM SYSTEM USER FEEDBACK SURVEY …………………..... 225

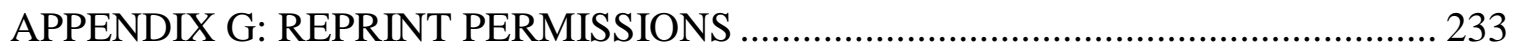




\section{LIST OF TABLES}

Table 1.1- Coronary stent type, material and mechanism of expansion......................... 30

Table 1.2- Coronary stent diameters, lengths, coatings and drugs .............................. 40

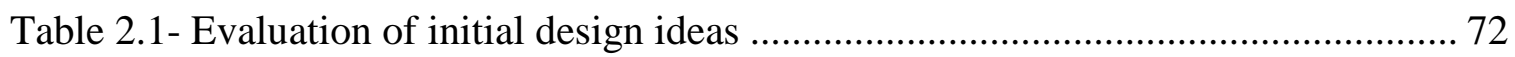

Table 2.2- Evaluation of design prototypes versus original BVM system ..................... 79

Table 3.1- Scaffold length versus number of couplers used ....................................... 99

Table 3.2- Total cost per individual completed Ideal BVM system ............................ 112

Table 3.3- Total cost per individual completed two-port media reservoir .................... 112

Table 3.4- Summary of user feedback survey ....................................................... 118 


\section{LIST OF FIGURES}

Figure 1.1- Atherosclerosis of the coronary arteries ................................................ 5

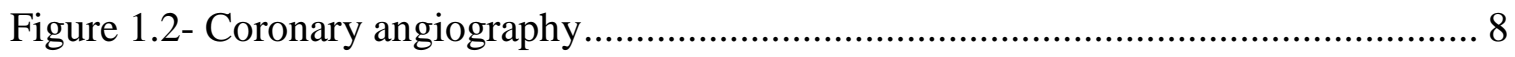

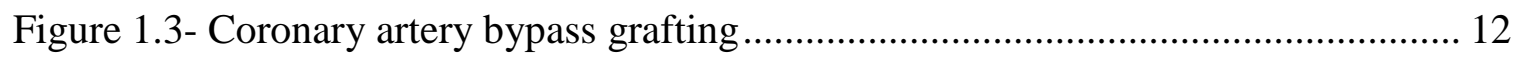

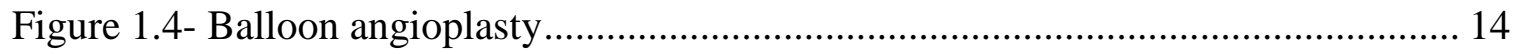

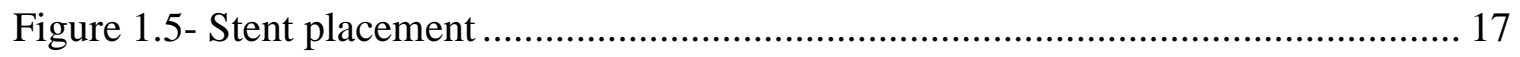

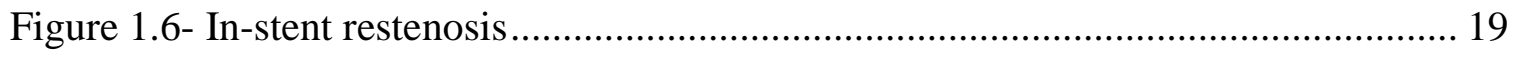

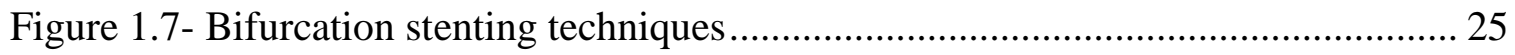

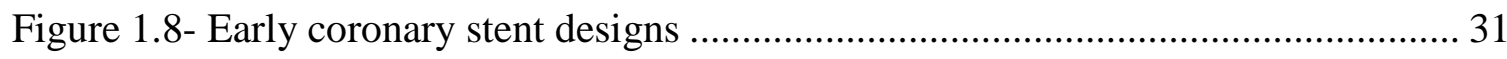

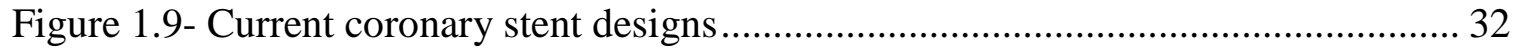

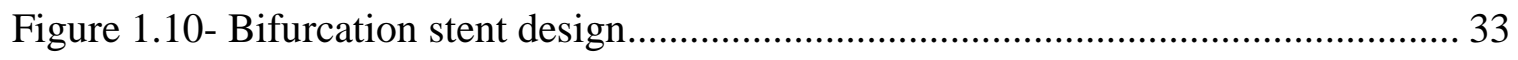

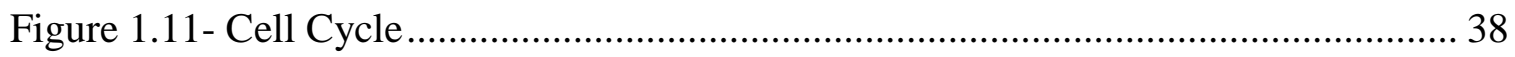

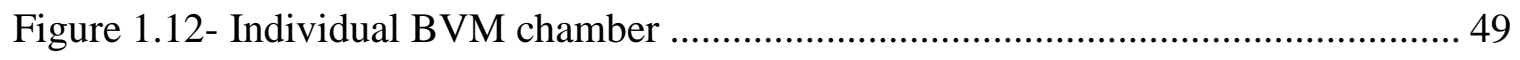

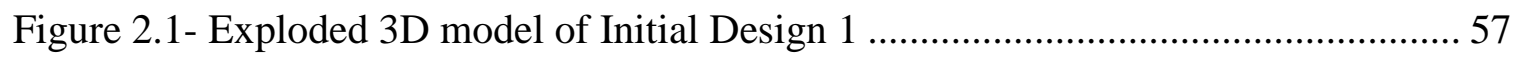

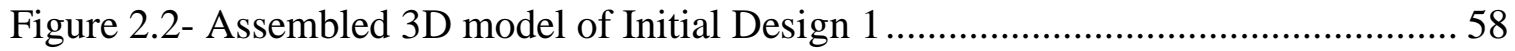

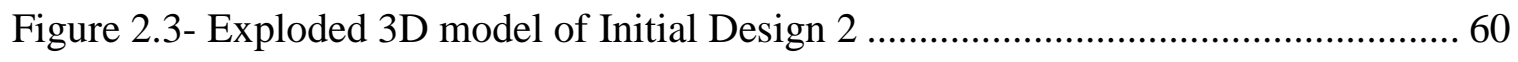

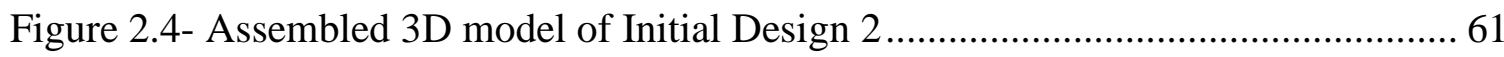

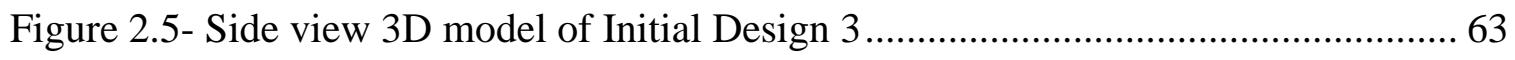

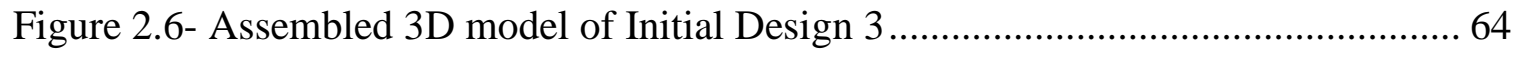

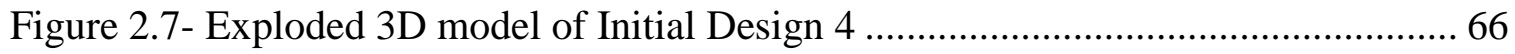




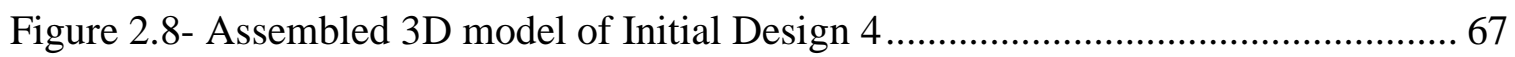

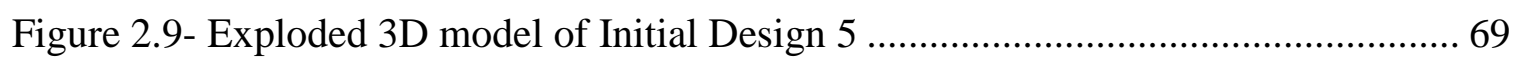

Figure 2.10- Assembled 3D model of Initial Design 5 ........................................ 70

Figure 2.11- Leak-proof plastic food container with snap-on sealing lid ...................... 74

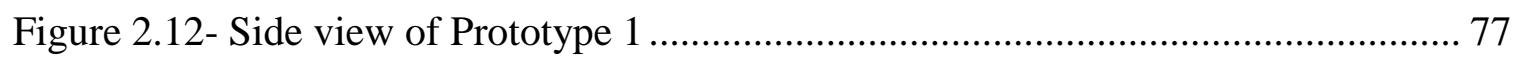

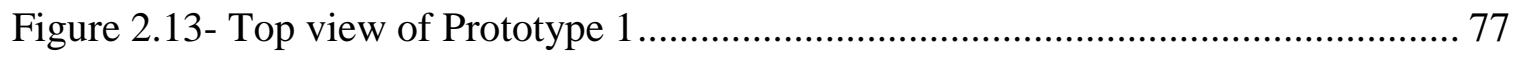

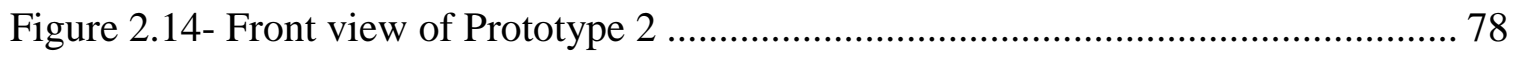

Figure 3.1- Assembly of the luminal inlet and luminal outlets................................... 85

Figure 3.2- Completed assembly of the luminal inlet and luminal outlets ..................... 86

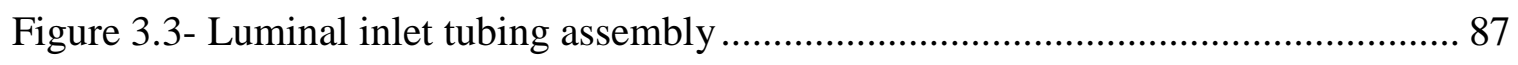

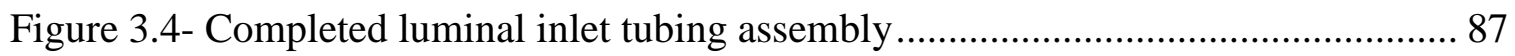

Figure 3.5- Overall view of luminal outlet and extra luminal outlet assembly ............... 89

Figure 3.6- Detailed view of luminal outlet and extra luminal outlet assembly .............. 89

Figure 3.7- Completed luminal outlet and extra luminal outlet assembly ..................... 90

Figure 3.8- Attachment of male-to-male luer lock connectors .................................. 91

Figure 3.9- Completed attachment of male-to-male luer lock connectors ....................... 91

Figure 3.10- Addition of male-to-female luer lock couplers ...................................... 92

Figure 3.11- Attachment of male-to-female luer lock couplers.................................. 92

Figure 3.12- Final complete new Ideal BVM system ............................................. 93

Figure 3.13- Assembly of two-port media reservoir lid .......................................... 95

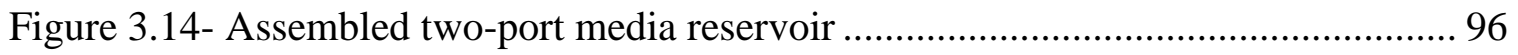


Figure 3.15- Inlet and outlet tubing assembly of two-port media reservoir 97

Figure 3.16- Final completed assembly of two-port media reservoir ........................... 97

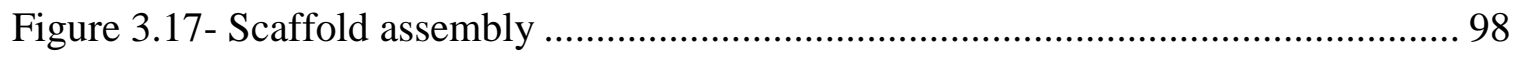

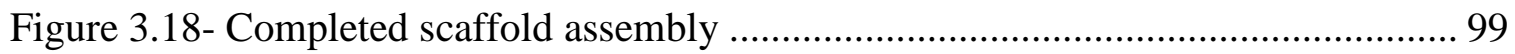

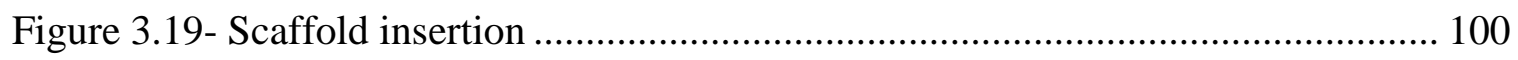

Figure 3.20- Ideal BVM system bioreactor configuration ..................................... 101

Figure 3.21- Ideal BVM functional verification setup.......................................... 102

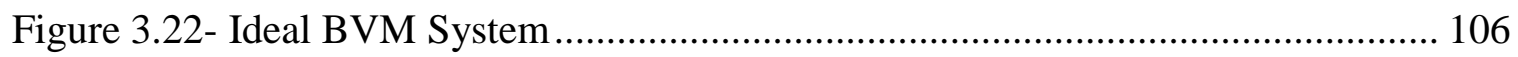

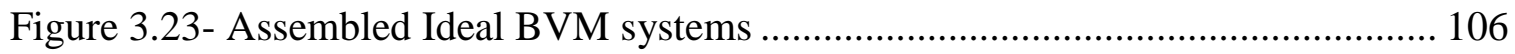

Figure 3.24- Two-port media reservoir............................................................. 107

Figure 3.25- Assembled two-port media reservoirs............................................. 108

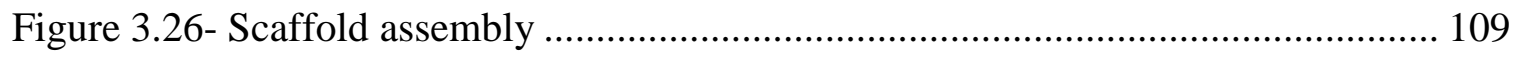

Figure 3.27- Scaffold insertion into Ideal BVM system........................................ 109

Figure 3.28- Complete bioreactor configuration ............................................... 111

Figure 3.29- Setup of the Ideal BVM bioreactor system ....................................... 113

Figure 3.30- Attachment of the Ideal BVM bioreactor system to a peristaltic pump..... 114

Figure 3.31- Two-port media reservoirs in use ................................................... 116

Figure 3.32- Side-by-side setup of original BVM and Ideal BVM systems................. 117

Figure 3.33- Side-by-side setup of original BVM and Ideal BVM bioreactors............. 117

Figure 3.34- SEM image of bare ePTFE scaffold surface ...................................... 120

Figure 3.35- SEM images of ePTFE scaffold surface with cells .............................. 121

Figure 3.36- Vertical bioreactor chambers ...................................................... 124

Figure 3.37- Horizontal bioreactor systems ....................................................... 125 
Figure 4.1- Coronary artery geometries

Figure 4.2- X-ray angiogram image of the coronary arteries 130

Figure 4.3- Bifurcated coronary artery geometries ............................................... 131

Figure 4.4- X-ray angiogram image of bifurcated coronary arteries ........................... 132

Figure 4.5- BVM system for long vessels ........................................................ 135

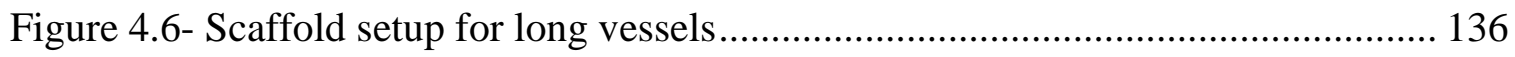

Figure 4.7- BVM system for slightly bent vessels .............................................. 138

Figure 4.8- Scaffold setup for non-angulated vessels ............................................ 138

Figure 4.9- BVM system for moderately angled vessels ....................................... 139

Figure 4.10- Scaffold setup for moderately angulated vessels ................................. 139

Figure 4.11- BVM system for severely angled vessels............................................ 140

Figure 4.12- Scaffold setup for severely angulated vessels ................................... 141

Figure 4.13- BVM system for Y-shaped bifurcation vessels................................... 143

Figure 4.14- Scaffold setup for Y-shaped bifurcation vessels .................................. 144

Figure 4.15- BVM system for T-shaped bifurcation vessels .................................. 145

Figure 4.16- Scaffold setup for T-shaped bifurcation vessels ................................. 145

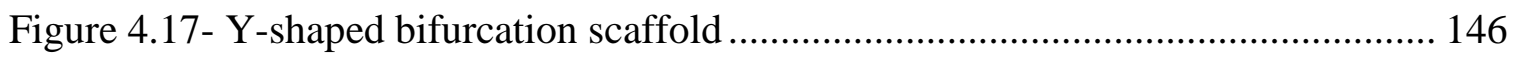

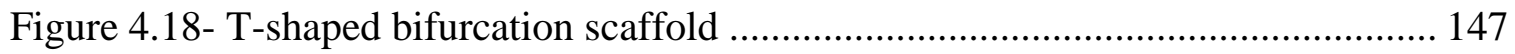

Figure 4.19- Multipurpose BVM System with a slightly bent scaffold...................... 150

Figure 4.20- Multipurpose BVM System with a moderately angulated $90^{\circ}$ scaffold .... 151

Figure 4.21- Multipurpose BVM System with a severely angulated $180^{\circ}$ scaffold ....... 152

Figure 4.22- Multipurpose BVM System with a T-shaped bifurcation scaffold ........... 153

Figure 4.23- Y-Shaped Bifurcation BVM System with scaffold................................ 155 


\section{INTRODUCTION}

Overview

Coronary stent implantation is being performed in a large number of patients with a wide spectrum of clinical and lesion characteristics. From single and multiple vessel disease to bifurcated, angulated and long lesions, the variation and complexity of coronary artery disease and the advanced capabilities to detect and determine the extent and severity has led to an increased interest in intravascular device innovation (Zhu, et al. 2008; Kini 2006). A variety of stent designs currently exist and continuous efforts are being made to improve both stent design and placement procedures (Kandzari, Tcheng and Zidar 2002; Kraitzer, Kloog and Zilberman 2008). Smaller delivery catheters and advanced delivery systems are also being introduced and have allowed the treatment of coronary artery disease and stent placement to become less invasive and easier on both the patient and physician (Yamasaki, et al. 2008; Takeshita, et al. 2008).

With the transition from open heart surgery to balloon angioplasty and stent placement, minimally invasive procedures have become the most often used technique for treating many cardiovascular diseases (American Heart Association 2008), which in turn has lead to a high demand for preclinical assessments of new intravascular devices (Kandzari, Tcheng and Zidar 2002; Cardinal, et al. 2006). The explosive growth of coronary stenting has fueled a surge in the number and types of coronary stents and delivery systems being designed and introduced to the market. Specifically in the area of coronary stents, a wide range of stent materials, coatings, drugs, and configurations currently exist, with future developments accelerating at a rapid pace (Kraitzer, Kloog 
and Zilberman 2008). Some of the most exciting advances can be seen in new stents designed specifically to treat bifurcation lesions, an area that has been very challenging for physicians, and new delivery systems which can deploy long or multiple stents at one time in order to better treat patients with long lesions or lesions in multiple vessels (Ikeno, Buchbinder and Yeung 2007; Stella, et al. 2008). This surge in stent technology has resulted in the need for new and advanced preclinical testing methods that provide medical device manufacturers and researchers with the ability to quickly evaluate tissue response to new and novel stent designs and stenting techniques.

Coronary stents are constantly evolving devices that possess great potential, but each innovation and each new design that is developed must be fully evaluated before clinical applications are possible. Through the creation of an in vitro tissue engineered blood vessel construct, previously developed and called a "blood vessel mimic" (BVM), a model system can be established for the initial testing of newly emerging intravascular technology (Cardinal, et al. 2006). The goal of this thesis is to not only expand upon the original BVM model, but to develop new and innovative models for the evaluation of devices in more complex geometries such as bifurcations, long and angulated vessels.

The following introduction will provide the background and foundation on which this thesis is based. A review of coronary artery disease will be given followed by current diagnosis and treatment procedures. Coronary stents will be discussed in detailed, outlining the materials utilized, shapes, configurations, coatings and drugs used in their design. Other intravascular devices, delivery systems and technologies will also be addressed along with an overview of intravascular device preclinical testing methods and the BVM system specifically. The introduction will conclude with a summary of the 
specific aims for this thesis, which will lead into the individual chapters and the models, experiments and studies that were built and performed. 


\section{Coronary Artery Disease}

Pathology

In order to understand why intravascular technologies, specifically stents, have become the treatment of choice for many cardiovascular diseases, and why the medical community has focused so much attention on creating and testing intravascular devices to treat these diseases, one must appreciate the history and prevalence of the disease itself. Coronary artery disease (CAD), also known as coronary heart disease (CHD), is the number one killer of both men and women in the United States, affecting an estimated 16 million people (American Heart Association 2008). In 2008 alone, the direct and indirect cost of treating CAD is estimated at $\$ 156.4$ billion (American Heart Association 2008), making it the primary focus of many medical device manufacturers.

The coronary arteries are small blood vessels that run along the surface of the heart and carry blood which supplies oxygen and nutrients to the heart muscle. The anatomical characteristics of coronary arteries vary considerably, ranging from straight simple geometries to extremely angulated bends and curves as well as bifurcations and long lesions (Ellis, et al. 1990; Zhu, et al. 2008; Smith, et al. 2001). Depending on the individual, the anatomical structure of the coronary arteries can include all or some of the characteristics identified above.

Coronary artery disease can be described as atherosclerosis of the coronary arteries (Falk 2006; Noll 1998). Atherosclerosis occurs when the coronary arteries become narrowed or blocked by fatty deposits called plaque, restricting blood flow to the heart (Deconinck, et al. 2008). A healthy artery and an artery affected by atherosclerosis 
are illustrated below in Figure 1.1. Without adequate blood, the heart becomes starved of oxygen and vital nutrients it needs to work properly. If left untreated coronary artery disease leads to chest pain, heart attack, heart failure and ultimately death (American Heart Association 2008). Therefore, action must be taken to reopen the narrowed vessels and restore blood flow.

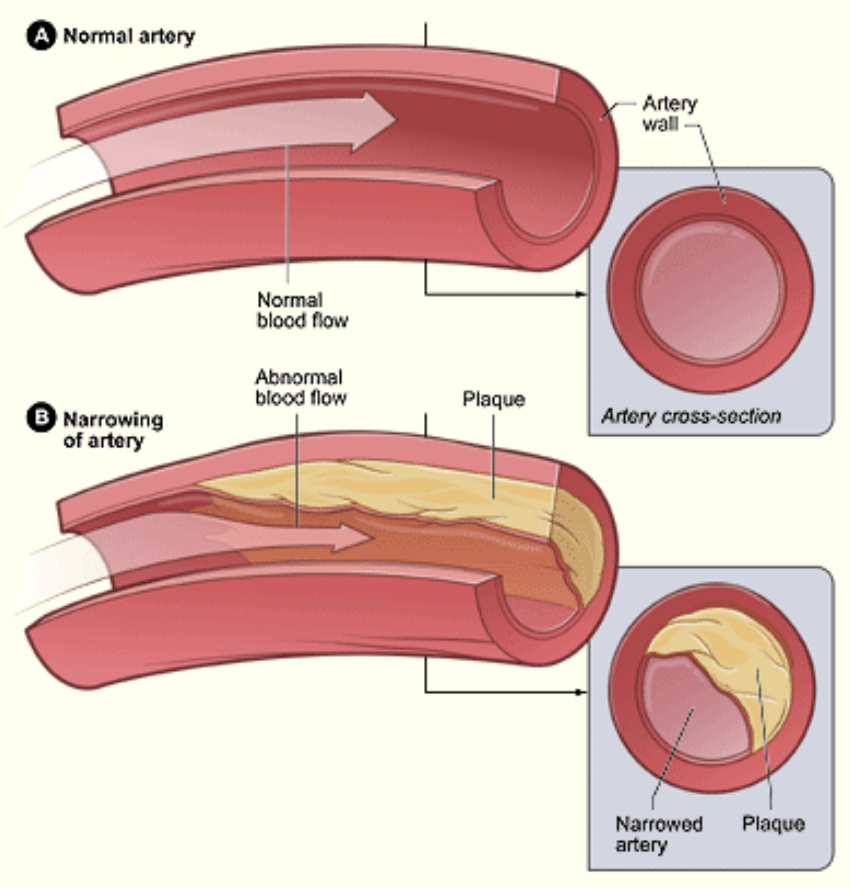

Figure 1.1- Atherosclerosis of the coronary arteries. Plaque accumulation on the artery wall results in restricted blood flow to the heart.

(Permission granted to reprint image from the National Heart, Lung, and Blood Institute as a part of the National Institutes of Health and the U.S. Department of Health and Human Services; see Appendix G) 
For many years, the process of atherosclerosis has been viewed simply as the accumulation of lipids within the artery wall, however it is now understood that atherosclerosis is a complex inflammatory disease (Libby, Ridker and Maseri 2002; Ross 1999; Noll 1998) involving endothelial cells (EC), smooth muscle cells (SMC), Tlymphocytes, B-lymphocytes, monocytes, leukocytes and macrophages (Stoll and Bendszus 2006; Falk 2006). This new insight into the role of inflammation in the development of atherosclerosis has initiated important new areas of research in the treatment of coronary artery disease, most notably in the area of pharmaceuticals (Deconinck, et al. 2008). As will be discussed in detail, the use of pharmaceuticals on the surface of stents in an attempt to prevent or slow the progression of atherosclerosis is a recent technology and an area that is still undergoing research and in need of preclinical testing options.

Diagnosis

Coronary artery disease develops slowly, often without any signs or symptoms prior to a heart attack. However, there are a number of symptoms that can help diagnosis CAD and lead to proper treatment (American Heart Association 2008). The most common symptom of coronary artery disease is chest pain, also known as angina pectoris, or simply angina (Khan 2005; Crawford 2002). Angina results from the heart not getting enough blood and oxygen and is described as chest discomfort, heaviness, tightness, pressure, aching, burning, numbness, fullness, or squeezing. It is most often felt in the chest, but may also be felt in the shoulders, arms, neck, back or jaw. Shortness of 
breath is a second symptom and is most often experienced during physical activity. Due to long-term lack of blood and oxygen, the heart becomes weak and is unable to pump blood throughout the body. Other less common symptoms of CAD include heart palpitations, irregular or rapid heartbeat, weakness, dizziness, nausea, and sweating. Whatever the symptom, diagnosis is the first step to treatment.

Diagnosis of coronary artery disease begins with a complete review of medical and family history and a thorough physical examination (Crawford 2002). Although both these methods can predict the likelihood of an individual's chance of having the disease a diagnostic test must be performed in order to verify the diagnosis. Often the first diagnostic tests chosen are an electrocardiogram (ECG or EKG) or echocardiogram (ECHO), since these tests are relatively low cost, simple, and non-invasive, providing fast information about the function of the heart. Stress tests, nuclear imaging, computerized tomography (CT) scans, positron emission tomography (PET) scans, magnetic resonance imaging (MRI) and other non-invasive diagnostic tests may also be performed, with varying degrees of imaging and informational capabilities. Currently, however, one of the most often used diagnostic tests and the reference against which all other diagnostic imaging tests are compared is the coronary angiogram, providing a direct image of the coronary arteries themselves (Budoff, et al. 2008; Sun and Jiang 2006). Coronary angiography is a minimally invasive imaging procedure in which a catheter is guided into the coronary arteries, a process often referred to as cardiac catheterization, dye is injected, and x-ray images are taken as the contrast material moves through the heart's chambers, valves and major vessels. The resulting images are used to identify sites of narrowing or blockage in the coronary arteries and provide a visual picture of the location 
and severity of coronary artery disease. Coronary angiography is illustrated below in Figure 1.2.

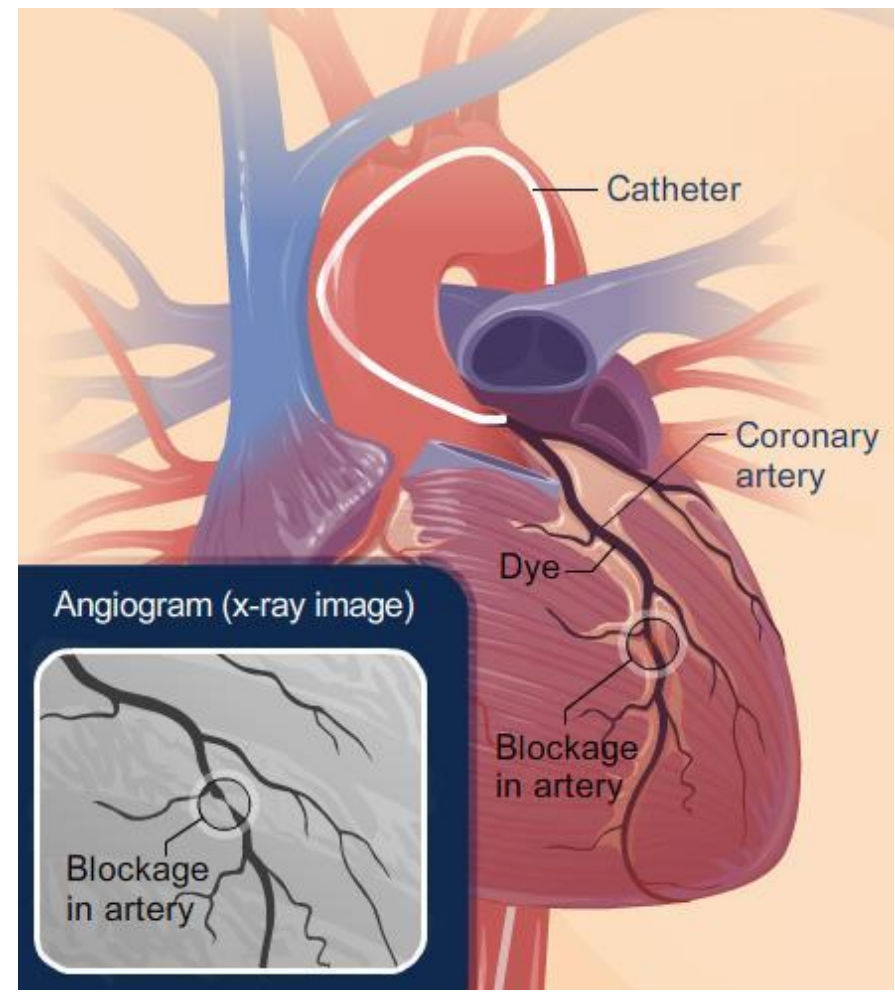

Figure 1.2- Coronary angiography. A catheter is guided into the coronary arteries, dye is injected, and x-ray images are taken as the contrast material moves through the heart.

(Permission granted to reprint image from the National Heart, Lung, and Blood Institute as a part of the National Institutes of Health and the U.S. Department of Health and Human Services; see Appendix G)

Using angiography alone, however, allows only a limited quantitative analysis of the vessel lumen blockage from the silhouette image obtained; and although angiography reveals artery narrowing, it cannot differentiate between the vessel layers or identify the presence of plaque on the artery walls (Kern, et al. 2006; Wahle, et al. 2006). Therefore, 
more advanced imaging techniques are often performed to supply additional information about the coronary arteries that angiography alone cannot provide.

Intravascular ultrasound (IVUS) is an imaging procedure that can be performed simultaneously with coronary angiography in order to obtain detailed images of the walls of the coronary arteries. During IVUS a miniature sound-probe is positioned on the tip of a coronary catheter. The catheter is threaded through the coronary arteries and using high-frequency sound waves detailed images of the inside walls of the arteries are created. These images show cross-sections of the lumen revealing the layered structure of the vascular wall and provide information about the composition of the lesions including plaque shape and size (Cardinal, et al. 2006; Schoenhagen and Nissen 2002).

No matter what technique is used, the goal of coronary imaging is to identify the location, characteristics, number and severity of lesions present in a patient diagnosed with coronary artery disease. Treatment decisions are based on these findings and specific treatment options are available for a number of lesion characteristics. Depending on the number, severity and location of the blockages found during the diagnostic procedure, the physician can recommend the appropriate treatment. 
Treatment of Coronary Artery Disease

Treatment of coronary artery disease can include lifestyle changes, medications, and medical procedures. The goal of each treatment is to relieve symptoms and reverse or slow the progression of the disease. Depending on the severity of the disease, a combination of treatments may be chosen. Although the focus of this thesis is on interventional medical procedures and intravascular devices, it is important to realize that if CAD is caught early enough interventional procedures or surgery may not be needed which is the best scenario for both the patient and physician.

\section{Lifestyle Changes and Medication}

Simple lifestyle changes can often help prevent or control CAD and for some individuals these changes may be the only treatment needed. It is hard to determine which controllable risk factors are most important but, in general, high cholesterol, cigarette smoking, excess weight, sedentary lifestyle, high blood pressure, and diabetes seem to be the most significant (American Heart Association 2008; Crawford 2002). Therefore, diet changes, avoiding tobacco products, exercise, and weight loss can often reduce these risk factors and control the onset and development of CAD.

For some individuals, however, lifestyle changes may not be enough and medication may be prescribed. A number of medications are currently used, each with the goal of either treating the risk factors associated with CAD or treating the effects of the disease. Aspirin, a blood thinner, is recommended for almost everyone who has CAD, to 
help reduce the risk of having a heart attack (Hayden, et al. 2002). Other medications include: anti-platelet agents and anticoagulants, which prevent the formation of blood clots and thin the blood; statins, which help lower cholesterol; nitrates and ACE inhibitors, which dilate the arteries and make it easier for blood to flow; beta-blockers and calcium channel blockers, which slow the heart rate and reduce blood pressure (Zoghbi, Dorfman and Iskandrian 2008). Unfortunately, when medications and lifestyle changes are not able to control symptoms or the narrowing progresses to a point that the heart muscle is at risk for damage, interventional procedures or surgery may be required.

\section{Coronary Artery Bypass Grafting}

A number of surgical procedures and interventional therapies have been developed over the past several decades to treat coronary artery disease, each with the goal of quickly and safely restoring blood flow to the heart. Coronary artery bypass graft surgery $(\mathrm{CABG})$ was developed as a treatment for CAD in the late 1960's and is still a frequently used technique today (American Heart Association 2008). In this procedure a healthy vein or artery is removed from the patient's body and used to bypass the coronary blockage (Khan 2005; Mullany 2003). The saphenous vein, internal mammary artery and radial artery are used most often, however when no healthy segment can be found within the patient's body a synthetic graft material, such as expanded polytetrafluoroethylene (ePTFE), can also be utilized (Weyand, et al. 1999).

During CABG the patient's chest is surgically opened and the harvested artery or vein is connected between the aorta and a healthy area of the heart in order to provide a 
pathway for the blood to flow around the site of the damaged and narrowed tissue. An illustration of CABG is shown below in Figure 1.3.

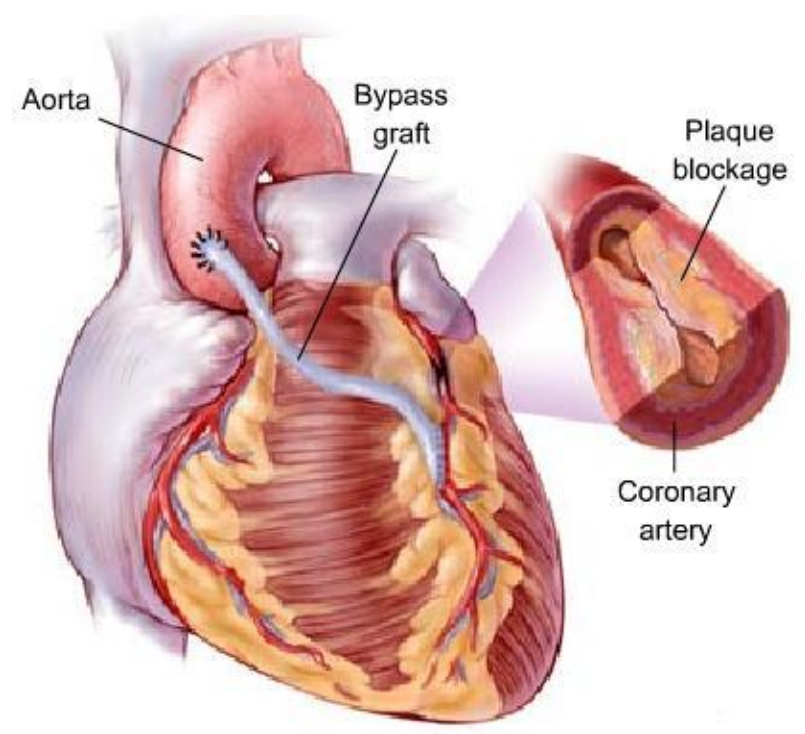

Figure 1.3- Coronary artery bypass grafting. A healthy vein or artery is connected between the aorta and a healthy area of the heart in order to bypass the coronary blockage and provide a pathway for blood to flow.

(Permission granted to reprint image from the American Heart Association/American Stroke Association; see Appendix G)

For many years, CABG had been considered the standard of care for treating CAD in patients at moderate to high risk of a heart attack (Hamm, et al. 1994). In many cases $\mathrm{CABG}$ is still the best choice of treatment. However, CABG can be a highly invasive procedure that is generally associated with long recovery times and hospital stays, as well as high cost (American Heart Association 2008; Mullany 2003). For many patients open heart surgeries, such as $\mathrm{CABG}$, are not well tolerated and lead to 
complications that are amplified by the heart's weakened condition. Thus less invasive methods to treat CAD have been developed.

\section{Balloon Angioplasty}

Percutaneous coronary intervention (PCI) is a minimally invasive procedure used to treat patients with diseased coronary arteries. The initial innovation was balloon angioplasty, originally referred to as percutaneous transluminal coronary angioplasty (PTCA). The first balloon angioplasty was performed in 1977 by the Swiss physician Andreas Gruentzig (King and Meier 2000; Gruentzig 1978) and the basic technique is still used today. During balloon angioplasty a physician inserts a flexible catheter with a balloon tip into the patient's vascular system, most often through the femoral artery at the groin, and maneuvers the catheter through the vascular system into the coronary arteries. At the site of the coronary lesion or blockage the balloon is inflated, compressing the plaque and stretching the artery wall, creating a wider channel and restoring blood flow to the heart. Balloon angioplasty is illustrated in Figure 1.4 below. 


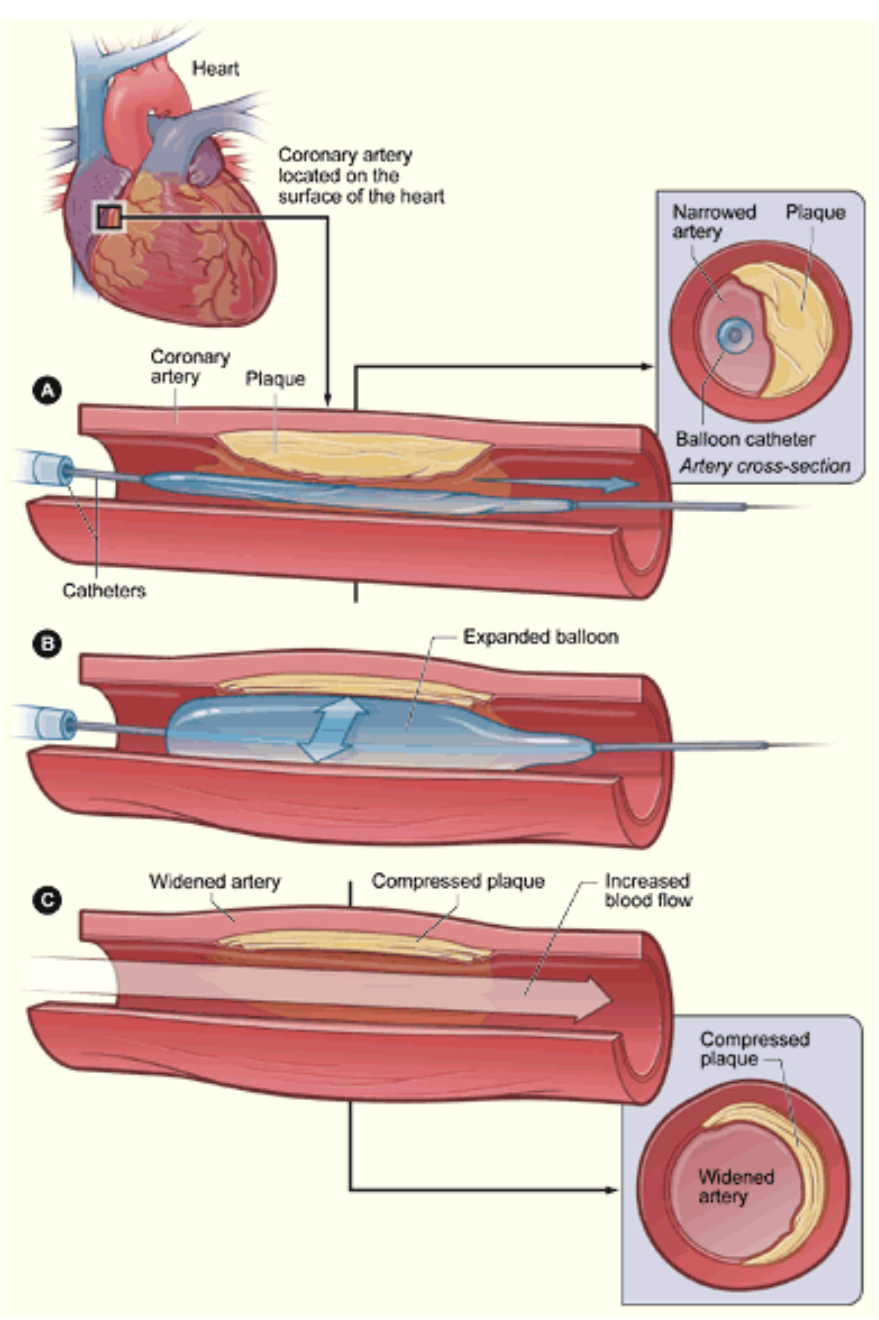

Figure 1.4- Balloon angioplasty. A flexible catheter with a balloon tip is maneuvered into the coronary arteries and inflated, compressing the plaque and stretching the artery wall restoring blood flow to the heart.

(Permission granted to reprint image from the National Heart, Lung, and Blood Institute as a part of the National Institutes of Health and the U.S. Department of Health and Human Services; see Appendix G) 
Coronary angioplasty provides physicians with a simple, minimally invasive method of opening a blocked artery without having to open the patient's chest. This therapy has been widely adopted by physicians because it results in shorter hospital and recovery times compared to $\mathrm{CABG}$ and is well tolerated by most patients (American Heart Association 2008). However, while providing advantages over CABG, the longterm effectiveness of balloon angioplasty is limited by restenosis, or re-narrowing of the artery. Restenosis is observed to occur due to two primary causes: the elastic recoil of the artery wall, its shrinkage back to its original size, and neointimal proliferation, the formation of scar tissue (Kraitzer, Kloog and Zilberman 2008; Deconinck, et al. 2008). This statement is an oversimplification of the process of restenosis, and restenosis will be discussed in detail in the following paragraphs, but for now it is important to note these two key observations as they are part of the motivation for the following advancements in treatment.

Stenting

One of most significant innovations in percutaneous coronary intervention, and that which is still evolving today, was the introduction of the stent in the late 1980's (Waksman 2002; King and Meier 2000). Stents are tube shaped scaffolds, typically metal or polymer, which are used to physically hold the artery wall open and address the first primary cause of restenosis (Kraitzer, Kloog and Zilberman 2008; Kandzari, Tcheng and Zidar 2002). In an attempt to prevent elastic recoil of the artery wall stents were incorporated into the balloon angioplasty procedure in the early 1990's. It is noteworthy 
to mention, however, that the stenting of peripheral arteries had already been experimented with by Charles Dotter in the 1960's who hypothesized that the use of stents in coronary arteries was also possible (King and Meier 2000; Dotter 1969). It should also be noted that in this section only a basic definition and description of stent design is addressed. Coronary stents will be discussed in detail in subsequent sections.

During a percutaneous coronary interventional stenting procedure, a stent mounted on a balloon catheter is delivered to the lesion, or site of narrowing and blockage. The balloon is inflated expanding the stent, compressing the plaque and stretching the artery wall. The balloon catheter is then removed, leaving the stent behind to hold the vessel open, restoring blood flow to the heart. Stent placement is illustrated below in Figure 1.5. 


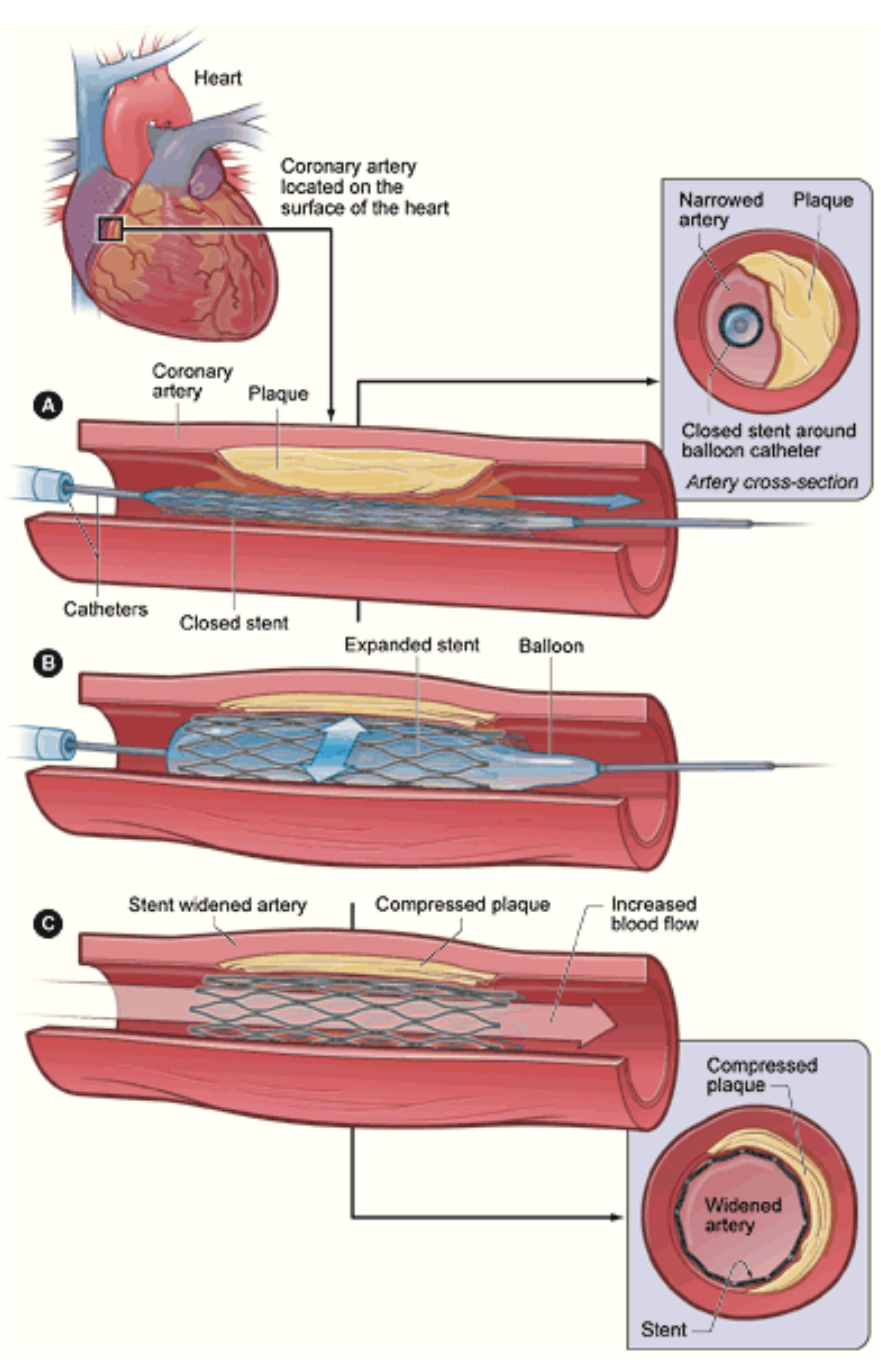

Figure 1.5- Stent placement. A stent is mounted on a balloon catheter and maneuvered into the coronary arteries. The balloon is inflated compressing the plaque, expanding the stent and restoring blood flow to the heart.

(Permission granted to reprint image from the National Heart, Lung, and Blood Institute as a part of the National Institutes of Health and the U.S. Department of Health and Human Services; see Appendix G) 


\section{Bare Metal Stents}

In 1986, Jacques Puel and Ulrich Sigwart inserted the first bare metal stent into a human coronary artery (King and Meier 2000; Sigwart, et al. 1987). Bare metal stents (BMS) are coil, mesh or slotted tubes consisting of interconnected struts that are inserted inside the narrowed artery and expanded to hold it open. Stents significantly lowered the occurrence of restenosis by preventing elastic recoil of the artery wall and quickly replaced the use of balloon angioplasty as the primary interventional therapy for CAD (Waksman 2002; King and Meier 2000).

In 1994 the first bare metal stents, Palmaz-Schatz and Gianturco-Roubin, were approved for use in the United States, and over the next decade, several generations of bare metal stents were developed. By early 2000, stenting was performed in over $80 \%$ of all PCI procedures reducing the restenosis rate to $15-20 \%$ (Holmes, et al. 2002; Waksman 2002; Kandzari, Tcheng and Zidar 2002). Previously, restenosis rates following balloon angioplasty had been seen in 30-40\% of patients (Silverman 2006; Waksman 2002) and often required re-intervention by physicians. Unfortunately, despite their widespread use, bare metal stents are plagued by the occurrence of in-stent restenosis, re-narrowing of the artery around the stent, with patients showing clinical evidence within the first 3-9 months after implantation (Holmes, et al. 2002). In-stent restenosis around an implanted stent is illustrated in Figure 1.6 below. 


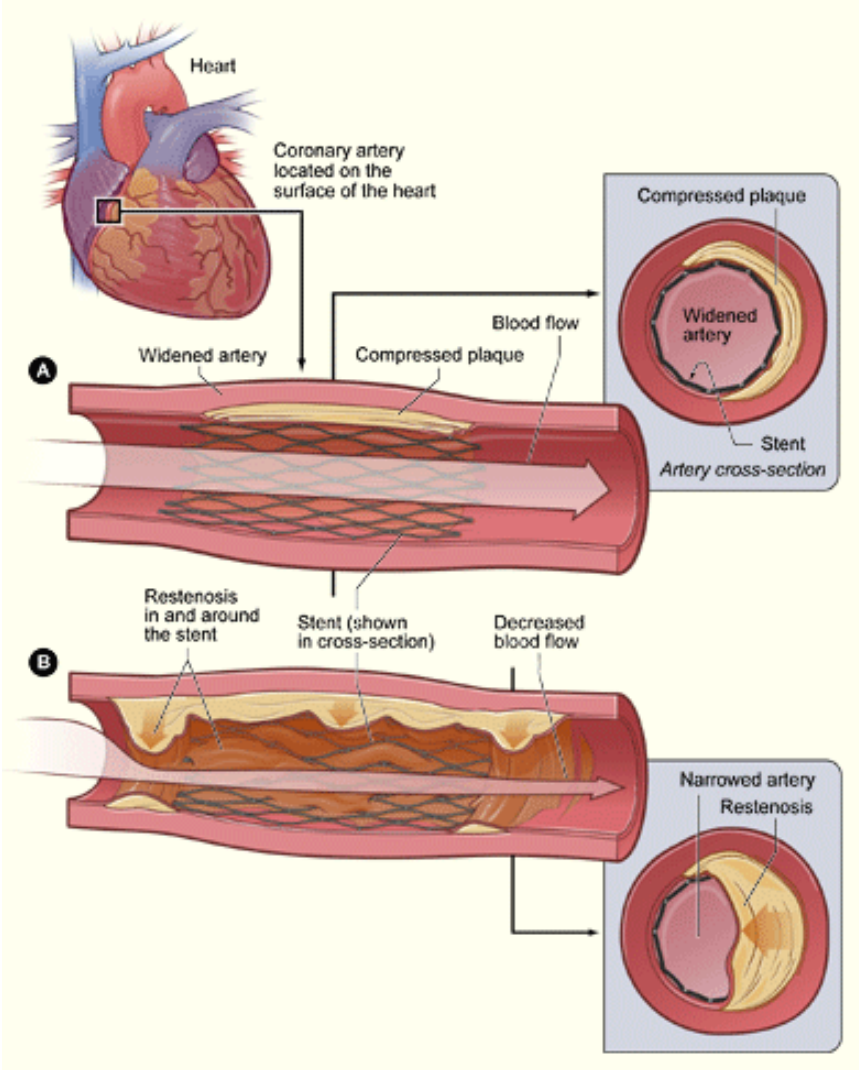

Figure 1.6- In-stent restenosis. Re-narrowing of the artery around the stent.

(Permission granted to reprint image from the National Heart, Lung, and Blood Institute as a part of the National Institutes of Health and the U.S. Department of Health and Human Services; see Appendix G)

Restenosis

Restenosis is defined as the arterial healing response after injury incurred during coronary angioplasty (Lowe, Oesterle and Khachigian 2002; Indolfi, et al. 2003). In-stent restenosis (ISR) is defined as the arterial healing response due to injury incurred during coronary angioplasty and the presence a stent. Both restenosis and in-stent restenosis are 
the result of a complex series of biological events in response to the initial injury of the vessel caused by balloon expansion and the presence of a permanent stent implant (Lewis, Tolhurst and Stratford 2002).

The process of restenosis involves vessel elastic recoil, negative remodeling, thrombus, smooth muscle cell proliferation, and excessive extracellular matrix production (Weintraub 2007). Neointimal formation, also known as neointimal proliferation, is one of the main mechanisms of restenosis and composed mainly of proliferating smooth muscle cells and extracellular matrix formation (Lowe, Oesterle and Khachigian 2002). In addition, inflammatory responses, foreign body reactions, and thrombogenesis occur in the tissue surrounding the struts of the stent and contribute to in-stent restenosis (Karoussos, et al. 2002). In-stent restenosis is a major clinical problem associated with stenting. Therefore, many efforts are being made to reduce or eliminate its occurrence.

In recent years, drug eluting, coated and bioabsorbable stents have been introduced into interventional cardiology as a possible solution to the problem of in-stent restenosis. Researchers and medical device manufacturers are continuously testing a variety of drugs that are known to interrupt the biological processes that cause restenosis (Deconinck, et al. 2008; Kraitzer, Kloog and Zilberman 2008). Stent coatings can optimize the surface properties of metallic stents and serve as a vehicle for local drug delivery (Hara, et al. 2006). Biodegradable materials can be absorbed by the body, allowing a stent to act as a scaffold for a period of time and then slowly disappear once it is no longer needed (Mani, et al. 2007). As a result the evolution of the stent has moved away from being a purely mechanical object placed within the artery. With 
pharmacologic and material advances, stents have become active devices that interact with their environment in complex ways.

\section{Drug Eluting Stents}

A recent innovation in percutaneous coronary intervention is the development of drug eluting stents (DES). Drug eluting stents address the second basic cause of restenosis by attempting to prevent or slow neointimal proliferation and the formation of scar tissue at the lesion site (Kraitzer, Kloog and Zilberman 2008; Waksman 2002; Lowe, Oesterle and Khachigian 2002). A drug eluting stent is a device that releases a single or multiple bioactive agents into the bloodstream that affect the tissue adjacent to the stent. The drugs used inhibit cell growth and proliferation and this advancement has resulted in improved patient outcomes due to reduced in-stent restenosis (Popma, et al. 2007). The specific drugs used to prevent restenosis and their incorporation into stent design will be discussed in detail in the following sections.

In 2001, drug eluting stents (DES) were introduced into percutaneous coronary interventional procedures as a strategy to minimize in-stent restenosis due to neointimal proliferation (Kraitzer, Kloog and Zilberman 2008; Deconinck, et al. 2008). In the data gathered so far drug eluting stents have been extremely successful in reducing in-stent restenosis rates from 15-20\% to single digits (Holmes, et al. 2002; Waksman 2002; Kandzari, Tcheng and Zidar 2002). At present, $90 \%$ of all stents placed in the United States are drug eluting (Popma, et al. 2007). One disadvantage, however, is the development of late in-stent restenosis and thrombosis, which occur after the drug has 
been completely eluted and is no longer present in the coronary stent (Deconinck, et al. 2008; Waksman 2007).

\section{Bioabsorbable Stents}

Despite the development and progression of bare metal and drug eluting stents, many concerns still remain regarding their permanent nature and influence on restenosis (Waksman 2007; Sharkawi, et al. 2007). Thus, bioabsorbable stents have emerged as an alternative to permanent metallic stents. Bioabsorbable stents, also described as biodegradable or bioresorbable, are constructed of metallic or polymer materials that degrade over time into substances that can be slowly absorbed by the body (Ormiston, et al. 2008; Erbel, et al. 2007). The specific materials used to create biodegradable stents will be discussed in the following sections. Bioabsorbable stents perform much like other coronary stents except that they are made of biodegradable materials that can be absorbed by the body, allowing them to initially act as supportive scaffolds while slowly disappearing as the vessel heals. The promise behind their technology lies in the fact that they provide a short term vessel scaffold, which can also be used for local drug delivery, and are then absorbed by the body avoiding the long term negative effects of permanent metallic stents such as in-stent restenosis and thrombosis. Currently biodegradable stents are not approved for clinical use in the United States (Ormiston, et al. 2008), however, clinical trials and research into their development are ongoing and release is expected in the near future. 


\section{Complex Lesions}

Many stents in the market today are designed and indicated for use in simple, straight, short lesions (Fournier, et al. 2005). However, in clinical practice coronary arteries are not straight and uniform. They run along the surface of the heart and are curved, bent, and angulated (Smith, et al. 2001). As a result, coronary stent implantation is being performed in an increasing number of patients with complex lesion characteristics including long, angulated and bifurcated lesions.

Coronary lesions are often extremely long in length and treatment can require multiple overlapping stents (Ruchin, et al. 2008). Therefore, new interlocking stent designs, such as the Custom NX(R) DES System manufactured by XTENT, Inc., are exploring the possibility of deploying stents up to $60 \mathrm{~mm}$ in total length (Stella, et al. 2008). It has also been observed that plaque accumulation is related to vessel curvature, with plaque accumulating more rapidly in angulated vessels where wall shear stress is low (Wahle, et al. 2006; Zhu, et al. 2008). Therefore, stents are most often placed in curved positions within the coronary arteries and remain in a bent position throughout their lifetime (Fournier, et al. 2005; Smith, et al. 2001). Many coronary stents, such as the MULTI-LINK manufactured by Abbott Vascular, CYPHER ${ }^{\circledR}$ manufactured by Cordis, and TAXUS ${ }^{\circledR}$ manufactured by Boston Scientific, claim superior flexibility and conformity which allow the stent to mold to the natural contours of the vessel wall (Serruys and Gershlick 2005; Rieu, et al. 2003). Consequently, accommodation of complex bends and geometries is being taken into greater consideration in overall stent design. 
A coronary bifurcation lesion is defined as a narrowing involving both the main vessel and a significant side branch (Louvard, et al. 2008). Bifurcation lesions account for $15-18 \%$ of all coronary lesions and represent one of the most complex coronary lesions to treat since the lumen of both the main vessel and side branch are affected and require treatment (Sukhija, Mehta and Sachdeva 2008). As described by the American College of Cardiology/American Heart Association (ACC/AHA) Guidelines for Percutaneous Coronary Intervention a coronary bifurcation occurs when one main vessel branches out into two smaller vessels, one being the continuation of the main vessel, and the other referred to as the side branch (Smith, et al. 2001). Recent advances have allowed physicians to treat bifurcation lesions, where both the main vessel and side branch need to be restored.

Many techniques, with the use of single or multiple stents, have been developed to treat bifurcation lesions (Sukhija, Mehta and Sachdeva 2008; Iakovou, Ge and Colombo 2005). The simplest approach is to stent the main vessel and dilate the side branch through the stent struts. A second approach is to use multiple stents and can be accomplished through a number of different techniques. V-stenting involves the delivery and implantation of two stents, one in each bifurcation branch. The simultaneous kissing technique is similar to V-stenting in that two stents are implanted, one in each branch, with the difference being that the two stents touch and extend into the main branch. Crush, T-stenting and modified T-stenting techniques require two stents to be positioned perpendicular to each other, and while the techniques vary, the resulting stent configurations are identical. Y-stenting involves the delivery and implantation of three stents, one in each bifurcation branch and one in the main branch. Finally, the skirt 
technique involves the delivery and implantation of one, two or three stents with the bottom half of the stent dilated to a larger diameter than the top. The bifurcation stenting techniques described are illustrated below in Figure 1.7.

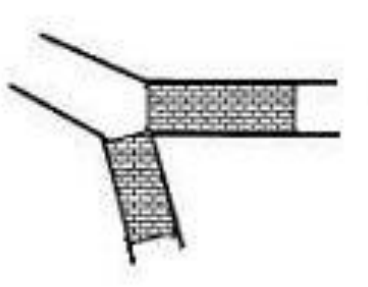

a)

b)
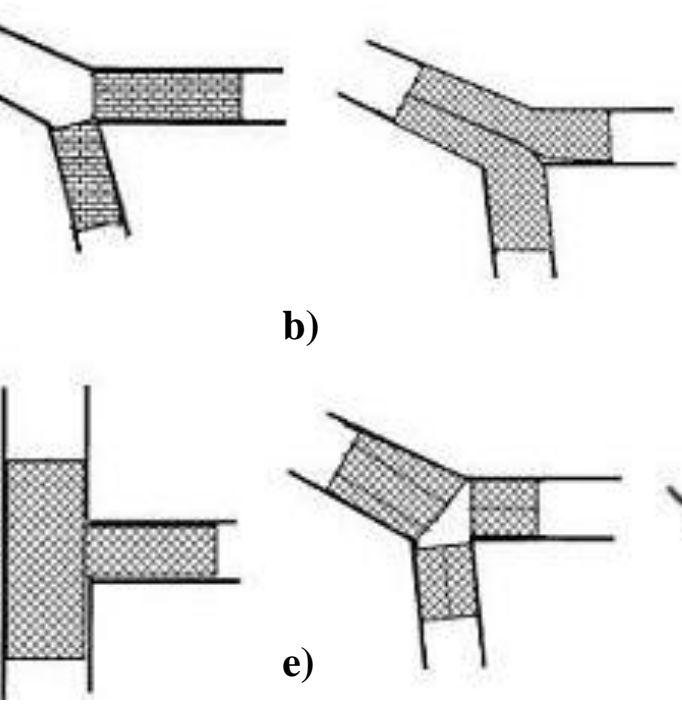

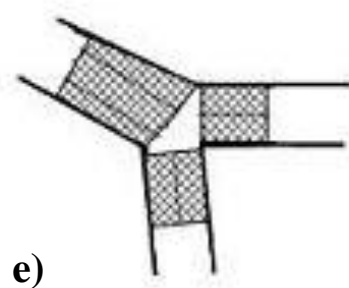

c)
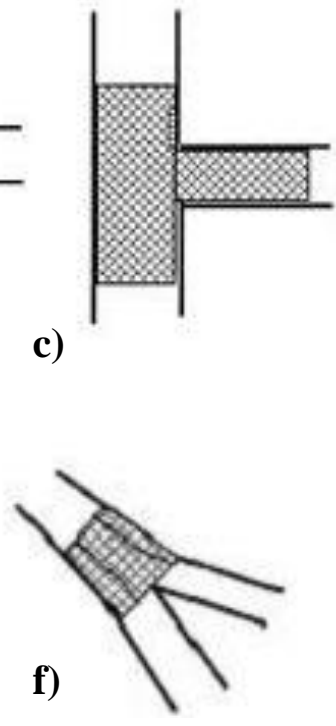

Figure 1.7- Bifurcation stenting techniques. V-stenting (a), simultaneous kissing (b), crush (c), T-stenting and modified T-stenting (d), Y-stenting (e) and skirt (f).

(Permission granted to reprint image from Iakovou, Ge and Colombo 2005 published in Journal of the American College of Cardiology by Elsevier Limited.; see Appendix G)

Most recently, novel stents and delivery systems such as AXXSSTM manufactured by Devax, Inc., PETAL ${ }^{\mathrm{TM}}$ manufactured by Boston Scientific, and MULTI-LINK Frontier manufactured by Abbott Vascular have been developed specifically to treat bifurcation lesions (Grube, et al. 2007; Ikeno, Buchbinder and Yeung 2007; Lefèvre, et al. 2005). These stents have the potential to provide physicians with a method to treat complex bifurcation lesions, resulting in better procedural outcomes and 
overcoming some of the technical difficulties associated with treating these complex lesions.

In summary, the variation and complexity of coronary artery disease and the advanced capabilities to detect and determine the extent and severity of coronary lesions has led to an increased interest in intravascular device innovation (Kini 2006). With the development of drug eluting and bioabsorbable stents, new drugs and materials are being introduced at an extremely rapid pace (Kraitzer, Kloog and Zilberman 2008). Each new drug and material must be evaluated for biocompatibility and tissue response and the competitive nature of biotechnology has the medical community searching for testing methods and procedures that provide rapid and reliable results.

The blood vessel mimic system has the potential to fulfill this preclinical testing need (Cardinal, et al. 2006). Ideally the BVM system will provide a means to rapidly evaluate human tissue response to varying stent configurations, materials, drugs and coatings as they become available. The safety of drug eluting and bioabsorbable stents, as well as stents designed specifically to treat complex lesions, is still an area of active research (Popma, et al. 2007). However, one thing remains clear- as the understanding of stent and tissue interactions continue to increase, the need to quickly evaluate new pharmacologic agents, configurations and materials will grow, and the need for a model system such as a BVM will be critical for preclinical device testing. 
Intravascular Technology

\section{Coronary Stents}

The types and construction of coronary stents is an area of active research and stents are constantly undergoing evolution in terms of the materials utilized, geometrical configurations, sizes, and coatings (Sangiorg, et al. 2007; Hara, et al. 2006; Kandzari, Tcheng and Zidar 2002). Stents have evolved from simple coil and slotted tubes to complex configurations with active coatings and advanced features. The current BVM system is a means of evaluating tissue response to varying stent surfaces, coatings, and

drugs as well as an evaluation of the physical stents themselves (Cardinal, et al. 2006). The BVM system is designed to test a variety of stent lengths and diameters with varying configurations and coatings, and knowledge of stent design is fundamental for understanding the needs of the system in order to physiologically mimic coronary arteries and induce representative cell response. Therefore, it is necessary to understand the general design and features of stents available in the market today.

\section{Mechanism of Expansion}

Stents can be divided according to their mechanism of expansion into two main categories: balloon-expandable and self-expanding (Kraitzer, Kloog and Zilberman 2008; Morton, Crossman and Gunn 2004). Balloon-expandable stents are made from materials that can be plastically deformed through the inflation of a balloon. The stent is mounted on the tip of a balloon catheter and the inflation of the balloon causes the stent to expand. 
Once the balloon is deflated the stent remains in its expanded shape inside the vessel. Self-expanding stents, on the other hand, are manufactured in their expanded shape, then compressed and constrained in a delivery system. Upon release from the delivery system they spring back to their manufactured shape. Both balloon-expandable stents and selfexpanding stents are readily available in the market; however, balloon-expandable stents are more common. A list of coronary stents and their mechanism of expansion is given in Table 1.1.

Materials

In order to provide effective treatment for coronary artery disease, the ideal stent material must be flexible, fatigue resistant, expandable, corrosion resistant, biocompatible and visible under standard medical procedures (Poncin and Proft 2003). Currently the most widely used material for coronary stents is stainless steel, typically 316L (Mani, et al. 2007; Sangiorg, et al. 2007; Hara, et al. 2006). Stainless steel is both biocompatible and corrosion resistant. In its fully annealed condition, stainless steel is easily deformable and the standard material for balloon-expandable stents. In its full-hard condition it can also exhibit enough elasticity for some self-expanding stent designs.

Although stainless steel is the most commonly used stent material, several other materials are currently used, with many more in development. A list of coronary stents and the material utilized for their construction is given in Table 1.1. The materials most commonly used by stent manufacturers today, after stainless steel, include cobalt chromium alloys, tantalum alloys, nitinol, and various polymers (Mani, et al. 2007; 
Morton, Crossman and Gunn 2004). Cobalt chromium is more biocompatible than stainless steel and is being increasingly used by coronary stent manufacturers (Hagemeister, et al. 2005). Cobalt chromium alloys are similar in strength to stainless steel, but have the advantage of being more radiopaque, allowing for the design of thinner struts without compromising visibility (Kandzari, Tcheng and Zidar 2002). Nitinol, an alloy of nickel and titanium, is also a common material for coronary stents (Poncet 2005). With its shape memory technology, nitinol is most often used in self-expanding stents.

Pure iron and magnesium alloys are two metals that are under development for use in bioabsorbable coronary stents (Mani, et al. 2007; Erbel, et al. 2007; Waksman 2007; Waksman, et al. 2008). Bioabsorbable metallic stents look promising but their long term biocompatibility still needs to be studied since the elevated local concentration effects of these elements is unknown. In addition to metallic bioabsorbable stent materials, polymers such as poly-L-lactic acid (PLLA), polyglycolic acid (PGA), polylactic-co-glycolic acid (PLGA), poly-D,L-lactic acid (PDLA) and polycaprolactone (PCL) show potential for use in bioabsorbable stents (Ormiston, et al. 2008; Sharkawi, et al. 2007; Waksman 2007). Further research is required, however, in order to better understand the varying tissue responses to these materials. 


\begin{tabular}{|c|c|c|c|c|c|}
\hline Stent Name & Manufacturer & Type & Material & $\begin{array}{l}\text { Expansion } \\
\text { Mechanism }\end{array}$ & Lesion Type \\
\hline $\begin{array}{c}\text { MULTI-LINK } \\
\text { VISION@ }\end{array}$ & Abbott & Bare Metal & Cobalt Chromium & $\begin{array}{c}\text { Balloon } \\
\text { Expandable }\end{array}$ & Small Vessel \\
\hline $\begin{array}{c}\text { MULTI-LINK } \\
\text { PIXEL®, ZETA } ®, \\
\text { ULTRA }^{\mathrm{TM}}\end{array}$ & Abbott & Bare Metal & Stainless Steel & $\begin{array}{c}\text { Balloon } \\
\text { Expandable }\end{array}$ & $\begin{array}{l}\text { Small Vessel } \\
\text { Large Vessel }\end{array}$ \\
\hline $\begin{array}{l}\text { MULTI-LINK } \\
\text { FRONTIER }\end{array}$ & Abbott & Bare Metal & Stainless Steel & $\begin{array}{c}\text { Balloon } \\
\text { Expandable }\end{array}$ & Bifurcation \\
\hline Dexamet & Abbott & Drug Eluting & Stainless Steel & $\begin{array}{c}\text { Balloon } \\
\text { Expandable }\end{array}$ & Small Vessel \\
\hline FlexMaster & Abbott & Coated & Stainless Steel & $\begin{array}{c}\text { Balloon } \\
\text { Expandable }\end{array}$ & Small Vessel \\
\hline TriMaxx & Abbott & Bare Metal & $\begin{array}{c}\text { Stainless Steel } \\
\text { Tantalum }\end{array}$ & $\begin{array}{c}\text { Balloon } \\
\text { Expandable }\end{array}$ & Small Vessel \\
\hline ZoMaxx & Abbott & Drug Eluting & $\begin{array}{c}\text { Stainless Steel } \\
\text { Tantalum }\end{array}$ & $\begin{array}{c}\text { Balloon } \\
\text { Expandable }\end{array}$ & Small Vessel \\
\hline XIENCE V & Abbott & Drug Eluting & Cobalt Chromium & $\begin{array}{c}\text { Balloon } \\
\text { Expandable }\end{array}$ & Small Vessel \\
\hline BVS & Abbott & $\begin{array}{l}\text { Bioabsorbable } \\
\text { Drug Eluting }\end{array}$ & Polylactic Acid (PLA) & $\begin{array}{c}\text { Balloon } \\
\text { Expandable }\end{array}$ & Small Vessel \\
\hline TAXUS® Express2 & $\begin{array}{c}\text { Boston } \\
\text { Scientific }\end{array}$ & Drug Eluting & Stainless Steel & $\begin{array}{c}\text { Balloon } \\
\text { Expandable }\end{array}$ & Small Vessel \\
\hline TAXUS® Petal ${ }^{\mathrm{TM}}$ & $\begin{array}{c}\text { Boston } \\
\text { Scientific }\end{array}$ & Drug Eluting & Platinum Chromium & $\begin{array}{c}\text { Balloon } \\
\text { Expandable }\end{array}$ & Bifurcation \\
\hline Express2 & $\begin{array}{c}\text { Boston } \\
\text { Scientific }\end{array}$ & Bare Metal & Stainless Steel & $\begin{array}{c}\text { Balloon } \\
\text { Expandable }\end{array}$ & Small Vessel \\
\hline Liberté & $\begin{array}{c}\text { Boston } \\
\text { Scientific }\end{array}$ & Bare Metal & Stainless Steel & $\begin{array}{c}\text { Balloon } \\
\text { Expandable }\end{array}$ & $\begin{array}{l}\text { Small Vessel } \\
\text { Large Vessel }\end{array}$ \\
\hline PROMUS & $\begin{array}{c}\text { Boston } \\
\text { Scientific }\end{array}$ & Drug Eluting & Cobalt Chromium & $\begin{array}{c}\text { Balloon } \\
\text { Expandable }\end{array}$ & Small Vessel \\
\hline BiodivYsio $^{\mathrm{TM}}$ & $\begin{array}{l}\text { Biocompatibles } \\
\text { Cardiovascular }\end{array}$ & Drug Eluting & Stainless Steel & $\begin{array}{c}\text { Balloon } \\
\text { Expandable }\end{array}$ & Small Vessel \\
\hline Whisper $^{\mathrm{TM}}$ & $\begin{array}{c}\text { Bioabsorbable } \\
\text { Therapeutics }\end{array}$ & $\begin{array}{l}\text { Bioabsorbable } \\
\text { Drug Eluting }\end{array}$ & Salicylic Acid & $\begin{array}{c}\text { Balloon } \\
\text { Expandable }\end{array}$ & Small Vessel \\
\hline CYPHER $®$ & Cordis & Drug Eluting & Stainless Steel & $\begin{array}{c}\text { Balloon } \\
\text { Expandable }\end{array}$ & Small Vessel \\
\hline AXXESSTM & Devax, Inc. & Drug Eluting & Nitinol & Self-Expanding & Bifurcation \\
\hline Endeavor & Medtronic & Drug Eluting & Cobalt Chromium & $\begin{array}{c}\text { Balloon } \\
\text { Expandable }\end{array}$ & Small Vessel \\
\hline Blazer & OrbusNeich & Bare Metal & Cobalt Chromium & $\begin{array}{c}\text { Balloon } \\
\text { Expandable }\end{array}$ & Small Vessel \\
\hline R-Stent & OrbusNeich & Bare Metal & Stainless Steel & $\begin{array}{c}\text { Balloon } \\
\text { Expandable }\end{array}$ & Small Vessel \\
\hline Carbostent $^{\mathrm{TM}}$ & $\begin{array}{c}\text { Sorin } \\
\text { Biomedica }\end{array}$ & Coated & Cobalt Chromium & $\begin{array}{c}\text { Balloon } \\
\text { Expandable }\end{array}$ & Small Vessel \\
\hline Custom NX(R) & Xtent & Drug Eluting & Cobalt Chromium & $\begin{array}{c}\text { Balloon } \\
\text { Expandable }\end{array}$ & Small Vessel \\
\hline
\end{tabular}

Table 1.1- Coronary stent type, material and mechanism of expansion.

Data gathered from references cited throughout text. 
Geometry and Configuration

Coronary stents come in a variety of different geometrical configurations. Early stent designs were generally classified as either slotted tube or coil geometries (Morton, Crossman and Gunn 2004). Slotted tube designs were simply metal tubes in which slots or sections were cut out, leaving open patterns. Coiled stents, on the other hand, were made of wire or sheet metal fashioned into tube-like structures. Early coiled and slotted tube stents are shown below in Figure 1.8.

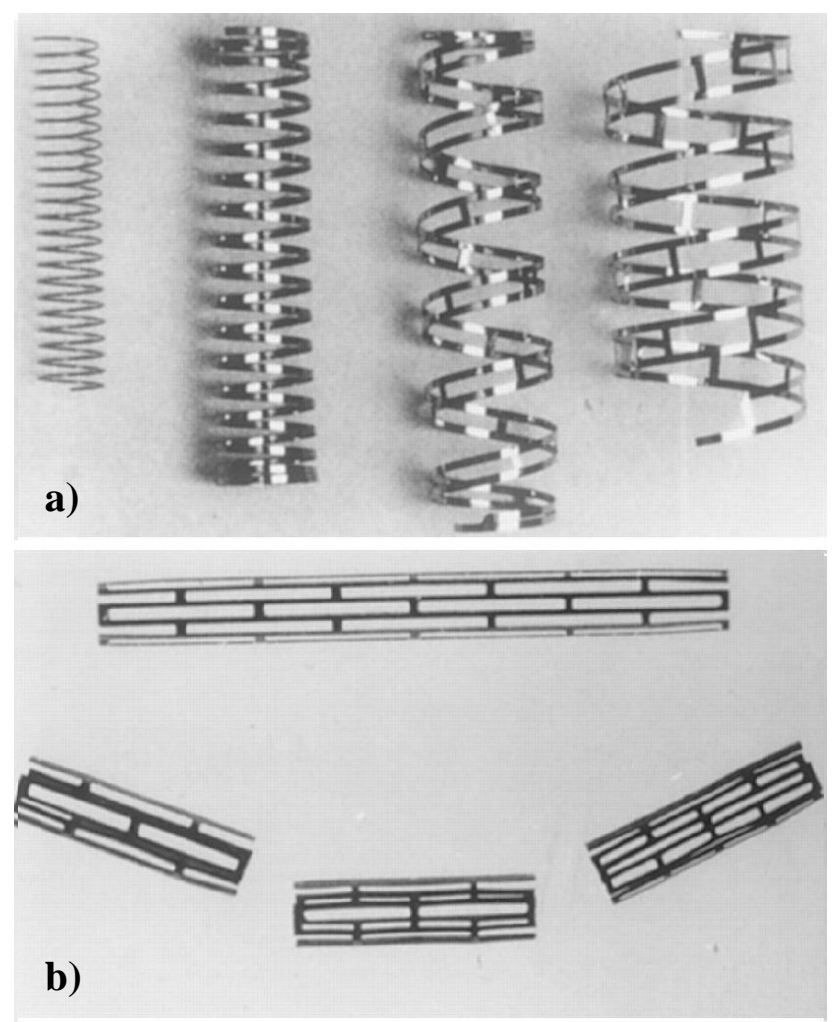

Figure 1.8- Early coronary stent designs. Coiled (a) and slotted tube (b) geometries.

(Permission granted to reprint image from Ruygrok 1997 published in The Asia Pacific Heart Journal by Elsevier Limited.; see Appendix G) 
While slotted tube designs had excellent radial strength, they lacked flexibility; the opposite occurred for coil designs (Sangiorg, et al. 2007; Morton, Crossman and Gunn 2004). The resulting evolution of stent design gave way to the development of a large variety of stent geometries and configurations including coils, wire mesh, slotted tubes, corrugated, and interconnected rings. Currently, the majority of stents in clinical practice are classified as slotted tube or cellular designs which are either laser-cut from a metal tube or composed of interconnected wire rings (Morton, Crossman and Gunn 2004; Lau, et al. 2004). Common stent geometries are shown below in Figure 1.9.

a)

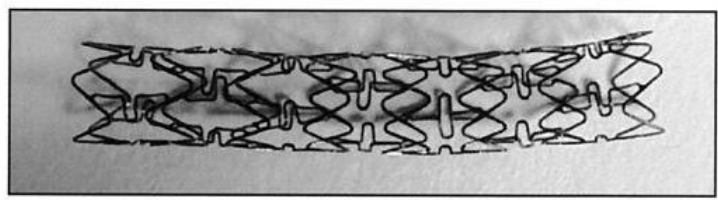

b)

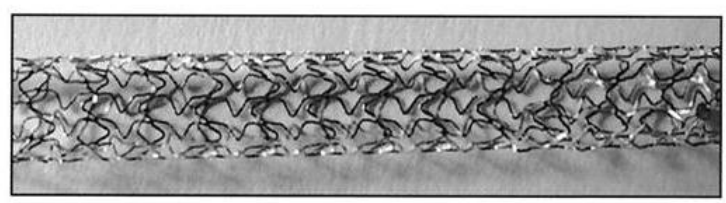

c)

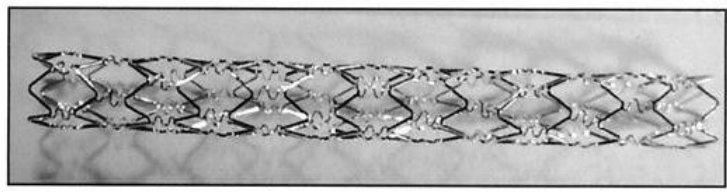

d)

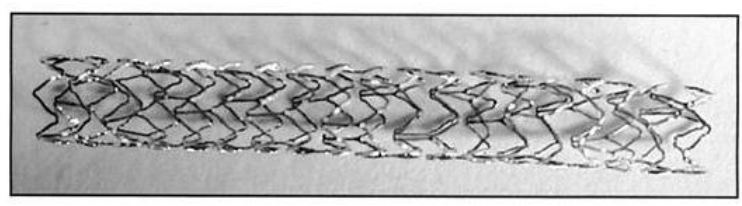

Figure 1.9- Current coronary stent designs. Sorin Carbostent (a), Medtronic beStent 2 (b), Cordis BxVelocity (c), and Boston Scientific Multilink Tetra (d).

(Permission granted to reprint image from Colombo, Stankovic and Moses 2002 published in Journal of the American College of Cardiology by Elsevier Limited.; see Appendix G) 
As stated in the previous section, novel stents have been developed specifically to treat bifurcation lesions (Ikeno, Buchbinder and Yeung 2007). These stents have varying configurations including branches, holes, and other geometrical features that allow for stenting of both the main branch and side branch. Several of the new bifurcation stent designs employ an innovative Y-shaped or T-shaped geometry in order to match the anatomy of lesions that form at the junctions of coronary arteries. The PETALTM bifurcation stent manufactured by Boston Scientific is shown below in Figure 1.10.

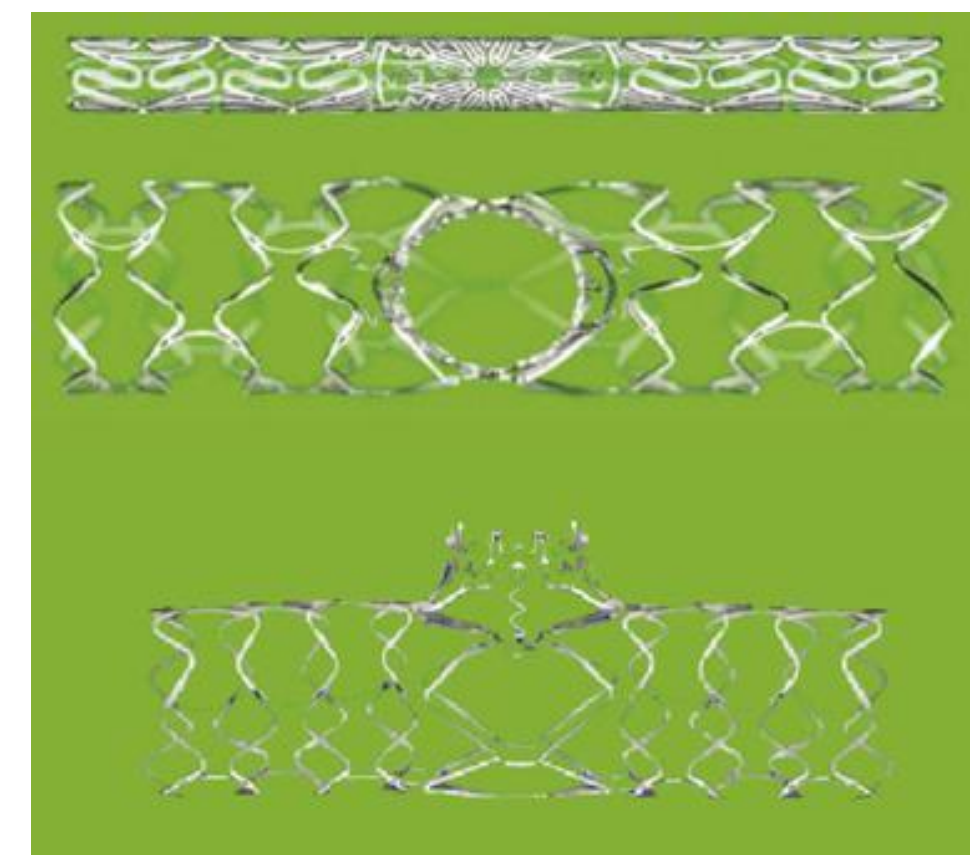

Figure 1.10- Bifurcation stent design. PETAL ${ }^{\text {TM }}$ bifurcation stent manufactured by Boston Scientific.

(Permission granted to reprint image from Ikeno, Buchbinder and Yeung 2007 published in Cardiovascular Revascularization Medicine by Elsevier Limited.; see Appendix G) 
Different stent designs yield varying tissue responses following implantation (Hara, et al. 2006) and reasearch concerning the relation of stent geometry, strut thickness and in-stent restenosis is ongoing. These design variations, as well as the desire to better understand the impact of stent characteristics on tissue response, result in a need for preclinical testing environments that support a range of stent shapes, geometries and configurations.

Diameter and Length

Coronary stents come in varying diameters and lengths (Kraitzer, Kloog and Zilberman 2008). A list of coronary stents along with their diameters and lengths is given in Table 1.2. The most common diameters available include: $2.00 \mathrm{~mm}, 2.25 \mathrm{~mm} 2.50$ $\mathrm{mm}, 2.75 \mathrm{~mm}, 3.00 \mathrm{~mm}, 3.50 \mathrm{~mm}, 4.00 \mathrm{~mm}, 4.50 \mathrm{~mm}$ and $5.00 \mathrm{~mm}$. The most common lengths include: $8 \mathrm{~mm}, 12 \mathrm{~mm}, 13 \mathrm{~mm}, 15 \mathrm{~mm}, 18 \mathrm{~mm}, 23 \mathrm{~mm}, 28 \mathrm{~mm}, 33 \mathrm{~mm}$, and 38 $\mathrm{mm}$. Some of the most recent designs, such as the Custom NX(R) DES System manufactured by XTENT, Inc., allow physicians to choose the appropriate stent length while inside the artery at the site of the lesion (Stella, et al. 2008). This variation results in a need for preclinical testing environments that support a range of stent sizes, ideally 2.0-5.0 mm diameters and 8-38 mm lengths (Kandzari, Tcheng and Zidar 2002; Kraitzer, Kloog and Zilberman 2008), in order to fit the variations of coronary arteries observed in clinical practice and the stents currently available in the market. 


\section{Coatings}

The surface properties of coronary stents significantly influence tissue response (Mani, et al. 2007). For this reason, coatings are often applied to metallic stents in order to change their surface properties and improve biocompatibility. These modifications can also inhibit neointimal proliferation and thrombosis, which can in turn reduce the rate of restenosis (Lau, et al. 2004). A list of coronary stents and the type of coating applied is given in Table 1.2.

The main advantage of a stent coating is the combination of mechanical stability and increased biocompatibility (Mani, et al. 2007). Coatings are a film of material bonded to the metallic surface of the stent, or embedded within the porous structure, and can be either passive or active. Passive coatings are inert and non-reactive in the body, while active coatings induce a biological response (Karoussos, et al. 2002). Many considerations must be taken into account when applying a stent coating including the biocompatibility, long-term effects, and material properties. In addition, it has been shown that stent coatings should not substantially enhance strut thickness because this promotes neointimal proliferation (Wieneke, et al. 2003).

Organic coatings include both biodegradable polymers, including PLLA, PGA, PLGA, PDLA, and PCL as discussed previously, and non-biodegradable polymers. Polymer coatings have been proposed to improve the biocompatibility of metallic stents and to serve as a matrix for drug delivery (Karoussos, et al. 2002). Phosphorylcholine (PC) based polymer coatings, unlike many other polymer coatings, do not provoke excessive inflammatory or adverse reactions and provide a useful drug delivery platform, 
especially for controlled release of drugs (Mani, et al. 2007; Waksman 2002). Other nonbiodegradable polymers for the potential use in controlled drug release include hyaluronic acid (HA), polyethylene-co-vinyl acetate (PEVA) and poly n-butyl methacrylate (PBMA).

There are many inorganic coating materials which are suitable as stents coatings. Gold, silicon carbide, aluminum oxide and diamond-like carbon are some of the most common (Mani, et al. 2007). Gold is highly radiopaque and has been shown to be very biocompatible and significantly reduce neointimal proliferation and thrombosis. However, clinical studies have shown that gold coated stents increase the risk of restenosis (Karoussos, et al. 2002; vom Dahl, et al. 2002). Silicon carbide is a passive stent coating, which has been shown to decrease thrombosis and increase biocompatibility. Even so, silicon carbide coatings do not significantly reduce restenosis and do not have any known clinical advantage over other stent coatings (Hara, et al. 2006). Porous ceramic coatings have become very promising for use as drug delivery platforms. The most recent development is a ceramic aluminum oxide coating with a unique nanoporous surface (Kang, et al. 2007; Wieneke, et al. 2003). Inorganic ceramic nanoporous aluminum oxide coatings have been shown to reduce neointimal proliferation and are specifically designed for incorporation of anti-restenosis drugs, which will be discussed in detail in the following sections. Lastly, carbon coated stents are coated with pure diamond-like carbon which is characterized by a polycrystalline structure and shown to improve biocompatibility (Mani, et al. 2007; Karoussos, et al. 2002). Currently, however, carbon coatings are not common in clinical use. 
Drugs

The ideal agent for incorporation into drug eluting stents should have potent antirestenosis effects while preserving vascular healing (Kraitzer, Kloog and Zilberman 2008). Anti-inflammatory, anti-thrombogenic, anti-proliferative, and immunosuppressive agents which interfere with early stages of the cell cycle are currently used in coronary stent designs to prevent or slow the progress of in-stent restenosis. Each of these agents inhibits one or more of the biological processes involved in restenosis and have revolutionized the treatment of coronary artery disease (Waksman 2002). Table 1.2 provides a list of coronary stents and the drugs incorporated into their designs.

Paclitaxel, synthetically produced as Taxol, is a potent inhibitor of cell proliferation and prevents mitosis of cells during the G2-M phase of the cell cycle (Hara, et al. 2006). The cell cycle consists of five basic steps: mitosis (M), gap phase 1 (G1), dormancy (G0), synthesis (S) and gap phase $2(\mathrm{G} 2) . \mathrm{G} 2-\mathrm{M}$ is the transition in the cell cycle from state $\mathrm{G} 2$, where the cell produces enough proteins to divide, to state $\mathrm{M}$, where the cell divides into two daughter cells. Paclitaxel acts by binding to microtubules during the G2 phase, thus preventing mitosis and cell replication. As a result, it inhibits smooth muscle cell proliferation and smooth muscle cell migration and has been shown to reduce inflammation (Deconinck, et al. 2008). Sirolimus, also known as rapamycin, its analogues zotarolimus and everolimus, and ABT-578 which is a synthetic analogue of rapamycin, block cell cycle progression at the G1-S transition (Kraitzer, Kloog and Zilberman 2008). G1-S is the transition in the cell cycle between phase G1, where the cell increases in size while producing proteins and RNA, to phase S when DNA 
replication occurs. As a result, it inhibits smooth muscle cell proliferation as well as Tcell and B-cell proliferation. Sirolimus and its derivatives are also known to prevent smooth muscle cell migration and reduce inflammation (Deconinck, et al. 2008; Mani, et al. 2007). An illustration of the cell cycle is shown below in Figure 1.11 identifying the cell cycle transition phase inhibited by both paclitaxel and sirolimus.

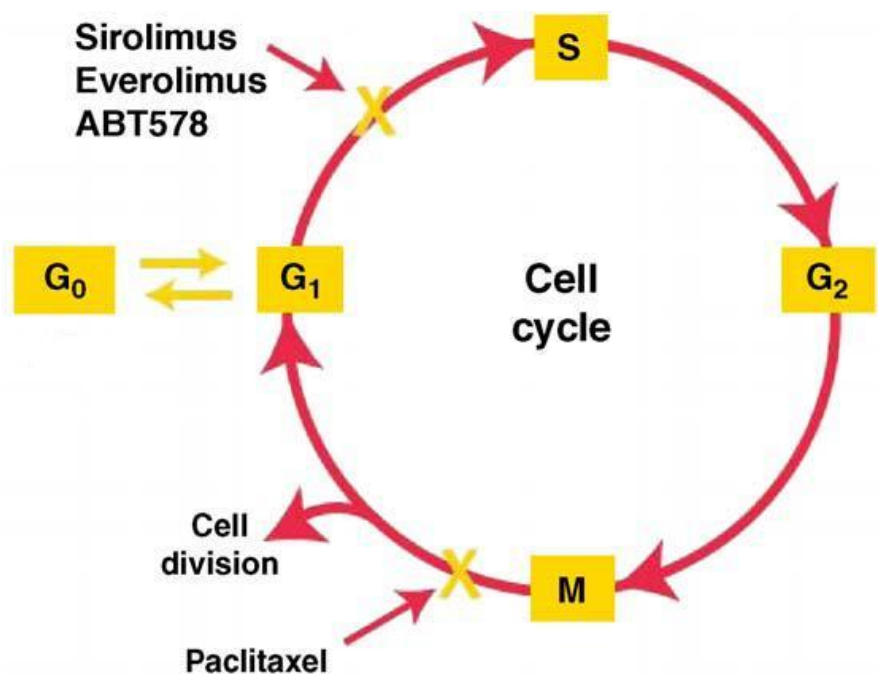

Figure 1.11- Cell Cycle. Sirolimus blocks cell cycle progression at the G1-S transition while paclitaxel prevents mitosis of cells during the G2-M transition.

(Permission granted to reprint image from Hara, et al. 2006 published in Advanced Drug Delivery Reviews by Elsevier Limited.; see Appendix G)

Other common agents for incorporation into drug eluting stents include tacrolimus (FK506), dexamethasone, and heparin (Deconinck, et al. 2008; Kraitzer, Kloog and Zilberman 2008). Tacrolimus and dexamethasone are both cellular proliferation inhibitors. Tacrolimus prevents the proliferation of T-cells, lymphocytes and smooth 
muscle cells. Dexamethasone is a well established anti-inflammatory agent and inhibits the proliferation of fibroblasts, smooth muscle cells and macrophages. Heparin, an anticoagulant, reduces leukocyte and platelet adhesion and protects against stent thrombosis (Mani, et al. 2007; Hara, et al. 2006). It also prohibits smooth muscle cell migration and proliferation.

Localized drug delivery using stents as the drug delivery platform is advantageous since the drug is release at the precise location and at the time of vessel injury (Kraitzer, Kloog and Zilberman 2008). In addition, higher local concentrations are achievable with little risk of system toxicity. Currently there are three different approaches to binding drugs to coronary stents: drug binding by means of a polymer on the stent surface, drug binding aided by an inorganic stent coating, or direct drug binding to the stent surface without coating (Acharya and Park 2006). Drugs are most often either chemically bonded to the surface of the stent or trapped within a polymer film or porous ceramic that allows controlled drug release (Kandzari, Tcheng and Zidar 2002). Drug release in biodegradable coatings is achieved through disintegration of the biodegradable polymer of the stent, while non-biodegradable coatings release drugs by allowing the drugs to diffuse through a porous matrix. 


\begin{tabular}{|c|c|c|c|c|c|}
\hline Stent Name & Manufacturer & Diameter (mm) & Length (mm) & Coating & Drug \\
\hline $\begin{array}{l}\text { MULTI-LINK } \\
\text { VISION® }\end{array}$ & Abbott & $\begin{array}{c}2.0,2.25,2.5 \\
2.75,3.0,3.5,4.0 \\
\end{array}$ & $\begin{array}{c}8,12,15 \\
18,23,28\end{array}$ & None & None \\
\hline $\begin{array}{c}\text { MULTI-LINK } \\
\text { PIXEL®, ZETA®, } \\
\text { ULTRA }^{\mathrm{TM}} \\
\end{array}$ & Abbott & $\begin{array}{l}2.0,2.25,2.5 \\
2.75,3.0,3.5 \\
4.0,4.5,5.0 \\
\end{array}$ & $\begin{array}{c}8,13,15,18 \\
23,28,33,38\end{array}$ & None & None \\
\hline $\begin{array}{l}\text { MULTI-LINK } \\
\text { FRONTIER }\end{array}$ & Abbott & $2.5,3 \cdot 0,3 \cdot 5,4.0$ & 18 & None & None \\
\hline Dexamet & Abbott & $\begin{array}{c}2.0,2.25,2.5 \\
2.75,3 \cdot 0,3.5,4.0 \\
\end{array}$ & $\begin{array}{c}7,8,10,11,15 \\
18,22,28 \\
\end{array}$ & Phosphorylcholine (PC) & Dexamethasone \\
\hline FlexMaster & Abbott & $\begin{array}{c}2.5,2.75 \\
3.0,3.5,4.0 \\
\end{array}$ & $\begin{array}{c}9,12,16 \\
19,23,26\end{array}$ & None & Heparin \\
\hline TriMaxx & Abbott & $\begin{array}{c}2.25,2.5,2.75 \\
3.0,3.5,4.0 \\
\end{array}$ & $\begin{array}{c}8,13,15 \\
18,23,28 \\
\end{array}$ & None & None \\
\hline ZoMaxx & Abbott & NA & NA & Phosphorylcholine (PC) & Zotarolimus \\
\hline XIENCE V & Abbott & $\begin{array}{c}2.5,2.75 \\
3.0,3.5,4.0\end{array}$ & $\begin{array}{c}8,12,15 \\
18,23,28\end{array}$ & $\begin{array}{c}\text { Polyvinylidene Fluoride } \\
\text { (PVDF) } \\
\text { Hexafluoropropylene } \\
\text { (HFP) }\end{array}$ & Everolimus \\
\hline BVS & Abbott & NA & NA & None & Everolimus \\
\hline TAXUS $®$ Express2 & $\begin{array}{c}\text { Boston } \\
\text { Scientific }\end{array}$ & $\begin{array}{c}2.5,2.75 \\
3.0,3.5 \\
\end{array}$ & $\begin{array}{c}8,12,16,20 \\
24,28,32\end{array}$ & $\begin{array}{c}\text { Polystyrene-b- } \\
\text { isobutylene-b-styrene }\end{array}$ & Paclitaxel \\
\hline TAXUS $®$ Petal $^{\mathrm{TM}}$ & $\begin{array}{c}\text { Boston } \\
\text { Scientific }\end{array}$ & NA & NA & $\begin{array}{c}\text { Polystyrene-b- } \\
\text { isobutylene-b-styrene }\end{array}$ & Paclitaxel \\
\hline Express2 & $\begin{array}{c}\text { Boston } \\
\text { Scientific }\end{array}$ & $2.5,2.75$ & $\begin{array}{c}8,12,16 \\
20,24\end{array}$ & None & None \\
\hline Liberté & $\begin{array}{c}\text { Boston } \\
\text { Scientific }\end{array}$ & $\begin{array}{c}2.75,3.0,3.5 \\
4.0,4.5,5.0 \\
\end{array}$ & $\begin{array}{c}8,12,16,20 \\
24,28,32 \\
\end{array}$ & None & None \\
\hline PROMUSTM & $\begin{array}{l}\text { Boston } \\
\text { Scientific }\end{array}$ & $\begin{array}{c}2.5,2.75 \\
3.0,3.5,4.0\end{array}$ & $\begin{array}{c} \\
8,12,15 \\
18,23,28\end{array}$ & $\begin{array}{c}\text { Polyvinylidene Fluoride } \\
\text { (PVDF) } \\
\text { Hexafluoropropylene } \\
\text { (HFP) }\end{array}$ & Everolimus \\
\hline BiodivYsio ${ }^{\mathrm{TM}}$ & $\begin{array}{l}\text { Biocompatibles } \\
\text { Cardiovascular }\end{array}$ & NA & NA & Phosphorylcholine (PC) & Dexamethasone \\
\hline Whisper ${ }^{\mathrm{TM}}$ & $\begin{array}{l}\text { Bioabsorbable } \\
\text { Therapeutics }\end{array}$ & NA & NA & None & $\begin{array}{c}\text { Sirolimus } \\
\text { Salicylic Acid } \\
\end{array}$ \\
\hline Conor Medstent ${ }^{\mathrm{TM}}$ & $\begin{array}{c}\text { Conor } \\
\text { Medsystems }\end{array}$ & NA & NA & $\begin{array}{c}\text { Polylactide-co-glycolic } \\
\text { Acid (PLGA) }\end{array}$ & Paclitaxel \\
\hline CYPHER $®$ & Cordis & $\begin{array}{c}2.5,2.75 \\
3.0,3.5\end{array}$ & $\begin{array}{c}8,13,18 \\
23,28,33\end{array}$ & $\begin{array}{l}\text { Polyethylene-co-vinyl } \\
\text { Acetate (PEVA) } \\
\text { Poly-n-butyl } \\
\text { Methacrylate (PBMA) } \\
\end{array}$ & Sirolimus \\
\hline ACHIEVETM & Cook & NA & NA & None & Paclitaxel \\
\hline AXXESS $^{\mathrm{TM}}$ & Devax, Inc. & NA & NA & Polylactic Acid (PLA) & Biolimus A9® \\
\hline Endeavor & Medtronic & NA & NA & Phosphorylcholine (PC) & Zotarolimus \\
\hline Blazer & OrbusNeich & $\begin{array}{c}2.5,2.75 \\
3.0,3.5,4.0\end{array}$ & $\begin{array}{c}9,13,15 \\
18,23,28,33 \\
\end{array}$ & None & None \\
\hline R-Stent & OrbusNeich & $\begin{array}{c}2.5,2.75 \\
3.0,3.5,4.0 \\
\end{array}$ & $\begin{array}{c}9,13,15 \\
18,23,28,33 \\
\end{array}$ & None & None \\
\hline Janus Carbostent ${ }^{\mathrm{TM}}$ & $\begin{array}{c}\text { Sorin } \\
\text { Biomedica } \\
\end{array}$ & NA & NA & Carbon & Tacrolimus \\
\hline Custom NX(R) & Xtent & NA & 36,60 & Polylactic Acid (PLA) & Biolimus A9® \\
\hline
\end{tabular}

Table 1.2- Coronary stent diameters, lengths, coatings and drugs.

Data gathered from references cited throughout text. 
There are many different stent coatings and drugs being researched today and only the most promising were addressed in this section. Those in clinical use at the present time are being perfected and many new and different coatings and drugs will be developed in the future. Tables 1.1 and 1.2 illustrate the various combinations of geometries, sizes, coatings, and drugs currently available in the market as well as those being designed for future clinical use. Many of these stents are currently undergoing clinical trials and are likely to be released in the near future. Others are still in the initial design phases and in need of a preclinical testing environment such as the BVM system.

Delivery Systems

In addition to the advancements in coronary stent design, the method of delivering these therapies has also improved. These improvements have made percutaneous coronary interventional procedures easier to perform and have reduced the amount of time for a single procedure. Over-the-wire delivery systems represented the first significant innovation in PCI procedures (Abrams, Baum and Pentecost 2006; Simpson, et al. 1982). Over-the-wire delivery systems allow manipulation of the guidewire independently from the balloon catheter. Rapid exchange, or monorail, delivery systems represent the next advancement and were developed to simplify the exchange of catheters by allowing a much shorter length of guidewire to be used. Rapid exchange delivery systems allow a single operator to manage both the catheter and the guidewire during a PCI procedure (Bonnett, et al. 2004). 
Stent delivery and implantation in complex coronary geometries is a challenge for conventional delivery systems. It is often difficult to maneuver 6 French catheters, the current standard for most stent delivery devices, through tortuous vessel geometries (Saeed, Banerjee and Brilakis 2008). The French catheter scale is commonly used to measure the outer diameter of cylindrical medical instruments, with one French equivalent to 0.013 inches. As a result, a novel 4 French balloon catheter was designed for the treatment of a variety of coronary artery lesions and provides a less invasive method of treatment while improving procedural outcomes (Takeshita, et al. 2008). The CardioMind Sparrow ${ }^{\mathrm{TM}}$ coronary stent delivery system manufactured by CardioMind is another low profile delivery device consisting of a self-expanding nitinol stent loaded into a 0.014 inch guidewire platform with the ability to travel through tortuous geometries (Yamasaki, et al. 2008; Abizaid, et al. 2007).

In addition to tortuous vessel geometries and the need for lower profile and more flexible delivery systems, complex coronary lesions often require high-pressure balloon inflation for complete stent and lumen expansion. The use of high-pressure balloon inflation has been associated with an increased incidence of restenosis due to injury caused by balloon inflation (Oesterle, et al. 1998). Therefore, novel low-pressure dilation balloon catheters, such as the FX miniRAIL manufactured by X Technologies, are being explored as a method to reduce the balloon pressure required for stent expansion in complex coronary lesions (Ischinger, Solar and Hitzke 2003).

Percutaneous coronary intervention in complex coronary geometries is difficult and the increased complexity results in reduced success rates (Saeed, Banerjee and 
Brilakis 2008). Therefore, novel delivery systems, smaller catheters, lower profile balloons and other devices have been created to overcome the challenges associated with tortuous anatomies. Stent delivery is an area of active research and a living model system for testing and evaluating these new delivery devices would be ideal for development and progression of the field. 
Preclinical Device Testing

Preclinical device testing is a crucial step in the process of developing and releasing new medical devices to the market. The objective of preclinical testing is to provide preliminary evidence of product safety and efficacy in order to support the initiation of human clinical trials (Wall and Shani 2008). Preclinical testing typically begins using models and methods that are simple and cost-effective in order to identify potential failures as early in the development process as possible, and then progresses to models with increasing complexity. Preclinical intravascular device testing is currently performed using laboratory models, in vitro cellular models, and in vivo animal models and provides critical information about cellular and tissue responses as well as device success.

\section{Laboratory Models}

One of the first steps in preclinical intravascular device testing involves the use of laboratory models. Prior to determining the cellular response to a device research must be performed in order to verify device functionality. Laboratory models are often used to physically mimic the geometry of coronary arteries and allow a large number of device designs to be evaluated quickly and inexpensively. For example, synthetic models in which vessel shaped troughs are bored through plastic blocks are often constructed for preclinical testing of stents in order to simulate device expansion and delivery within a coronary vessel (Ormiston, et al. 1999). Other models use flexible tubing to mimic the vascular structure of the coronary arteries and foam-like material to represent and mimic 
the presence of a lesion (Connolley, et al. 2007). These types of synthetic model approaches allow stents to be deployed and evaluated in coronary geometries; however, no cellular lining exists to provide physiological response evaluations.

It is important to remember that stent implantation is a mechanical intervention and the engineering aspects and mechanical performance of the stent design must be evaluated. Methods for identifying mechanical complications enable preclinical screening of stent designs, which reduces the amount of animal testing required and provides a method for evaluating new stent designs and stenting techniques prior to animal and human clinical trials.

\section{In Vitro Cellular Models}

Following functional and mechanical testing in a laboratory model, in vitro cellular models are the next step in preclinical intravascular device testing. Although a more complex dynamic system exists in vivo, a common preclinical evaluation method involves culturing endothelial cells in vitro in order to study their response to new drugs, materials, and devices (Cenni, et al. 1999). These cells can be maintained as twodimensional monolayers in flasks or dishes, or cultured in three-dimension adhered to scaffolds or suspended in solution (Bokhari, et al. 2007; Cheng, Lai and Kisaalita 2008; Cui, et al. 2007; Sun, et al. 2006). Either of these cellular models can then be exposed to samples of new materials or medical devices in order to assess responses such as cell proliferation, cell migration, and cell death. Although in vitro cell cultures do not reflect the complexity of a living system, they provide important insights into cellular response 
and are critical in the effort to minimize the time and cost associated with in vivo animal and human studies.

\section{In Vivo Animal Models}

Currently most preclinical biocompatibility and tissue response testing is done on animals in outside laboratories, which is both time consuming and expensive. Animal studies, however, are a valuable tool and much of what is known about restenosis and the healing response after stent implantation has been gathered from the study of both healthy and atherosclerotic animal models (Touchard and Schwartz 2006; Schwartz, Chronos and Virmani 2004; Wieneke, et al. 1999).

The ideal animal model for preclinical intravascular device testing should be readily available, inexpensive, and easy to handle. More importantly, it should closely mimic the human pathophysiology of neointimal proliferation and remodeling (Russell and Proctor 2006; Kantor, et al. 1999). Current animal models for preclinical intravascular device testing include mice, rats, rabbits, pigs, dogs, and primates (Russell and Proctor 2006; Touchard and Schwartz 2006; Schwartz, Chronos and Virmani 2004).

The Apo E-deficient mouse model has become the most widely used animal model in atherosclerosis research (Desai, Zhao and Warren 2008; Fazio and Linton 2001). Apo E-deficient mice have been genetically altered so that they naturally develop high cholesterol, leading to atherosclerosis and the development of coronary lesions and blockages much like those found in human coronary arteries (Russell and Proctor 2006). 
The rat carotid artery and the rabbit iliac model are the most commonly used animal models for testing intravascular devices targeted at preventing restenosis. Rats and rabbits are small and relatively inexpensive, providing a fast and reliable animal model for preclinical testing. One disadvantage is that rats and rabbits are highly resistant to the development of atherosclerosis, necessitating the use of either a high cholesterol diet or vessel injury to mimic coronary lesions (Schwartz, Chronos and Virmani 2004; Wieneke, et al. 1999). However, even healthy rat and rabbit models provide important information regarding the vascular healing response that occurs after device implantation.

In addition to rats and rabbits, the pig has become a widely used species for assessing treatments of restenosis because its coronary anatomy and physiology are similar to those of humans and are susceptible to atherosclerosis, developing thick neointimal lesions in response to injury (Russell and Proctor 2006; Schwartz, Chronos and Virmani 2004). Large animas, such as dogs and primates, are also useful models but are not commonly used due to high cost, ethical considerations, and long life spans.

Although preclinical animal studies are accepted as a means to predict the probability of device safety and effectiveness in humans, no animal model can provide complete insight into the human healing response following stent implantation (Wall and Shani 2008; Fazio and Linton 2001; Wieneke, et al. 1999). The need exists for preclinical testing using live human cells and tissue. Preclinical testing using human cells would greatly increase the medical community's understanding of tissue response to a specific device prior to animal and patient testing and prior to device release. Presently, it is difficult to simulate device and tissue interaction and research still needs to be done to 
fully understand tissue behavior and the in vivo interaction that occurs between device and tissue.

\section{Blood Vessel Mimic}

Previous work has demonstrated that a 3-dimensional in vitro tissue engineered blood vessel mimic (BVM) can be created and maintained in a bioreactor system to serve as a potentially ideal environment for coronary stent implantation and testing prior to animal and clinical studies (Cardinal, et al. 2006). The in vitro blood vessel mimic system can allow initial preclinical testing to be performed on a large number of coating combinations and stent configurations in order to direct animal and clinical studies toward the most promising devices. The major advantage of the in vitro blood vessel mimic system is its potential for consistent high-throughput evaluation of cellular response to advancing intravascular technologies.

The in vitro blood vessel mimic design and concept is based upon existing techniques for tissue engineered vascular grafts and bioreactor systems. Human microvascular endothelial cells isolated from adipose tissue are pressure sodded onto tubular expanded polytetrafluoroethylene (ePTFE) scaffolds and cultivated in bioreactor systems under flow to form a "blood vessel mimic". Results demonstrate that a thin, confluent cellular lining on the inner lumen of the ePTFE scaffold can be created in vitro. Stent deployment within the blood vessel mimic is also possible and after being cultivated in the bioreactor system for several days a thin lining of cells forms over the stent surface. 
The BVM system described above (Cardinal, et al. 2006) consists of a custom built horizontal rectangular vessel chamber that houses an ePTFE scaffold. An individual BVM chamber is shown below in Figure 1.12. Following vessel cultivation and growth within the chamber, stents or other intravascular technologies can be evaluated and tested.

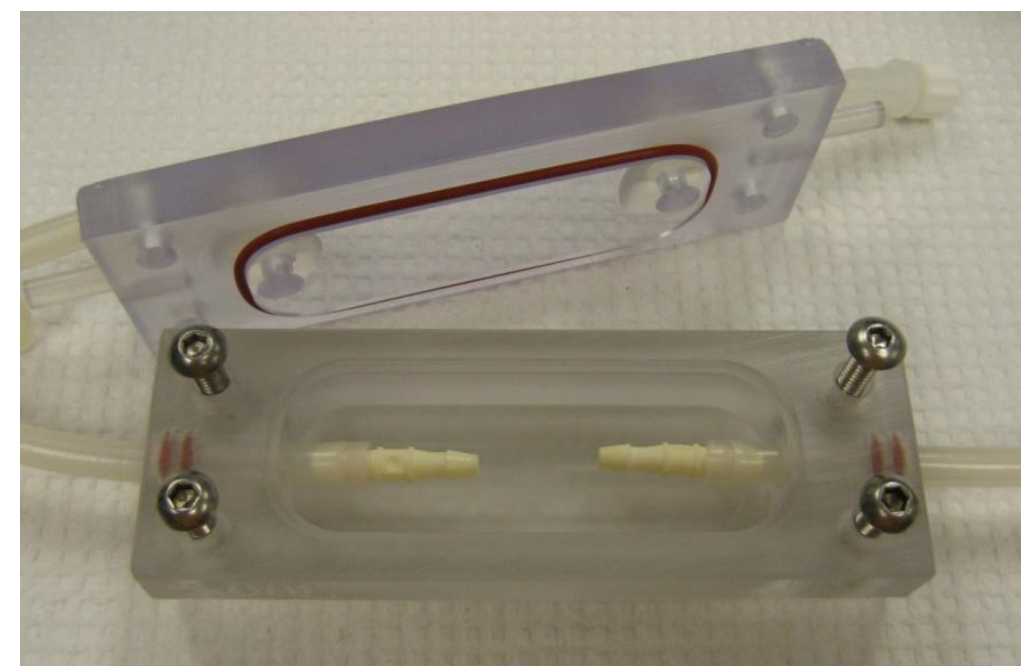

Figure 1.12- Individual BVM chamber.

The BVM system provides an intermediate testing environment for assessing human cellular responses to intravascular stents in a manner that combines the advantages of both in vitro and in vivo approaches. The blood vessel mimic system utilizes human cells, which provide the potential for more predictive preclinical results than animal models alone can provide. It is important to note that the blood vessel mimic system is not a substitute or replacement for animal models. The overall goal of the BVM system is to provide a more relevant in vitro tissue model that can be used for the preclinical evaluation of new intravascular devices. 
Summary and Aims of the Thesis

Coronary artery disease affects millions of people and the ability to detect and treat the disease is advancing at a rapid rate. Intravascular device technology is the focus of many medical device manufacturers and with coronary stent implantation being performed in an increasing number of patients a number of new stent designs have been introduced to the market, resulting in the need for improved preclinical testing methods.

A blood vessel mimic (BVM) system has previously been established and its feasibility for the initial testing of newly emerging intravascular technology has been demonstrated. The current BVM system has been used successfully to support the growth of tissue engineered in vitro blood vessel mimics, and its components and usefulness have been summarized. With that said, there are limitations that exist with the original design and the focus of this thesis is to both improve and expand upon the original model. The goal of this thesis is to develop a BVM system which has the advantages of simplicity and ease of assembly, as well as the ability to accommodate varying sizes of vessel scaffolds and to expand the BVM concept to an area that currently has not been addressed, such as long, angulated and bifurcation lesions, all while maintaining the needs of a tissue engineering bioreactor system.

The general hypothesis driving this thesis project was that design changes could be made and new configurations implemented to the BVM system that would improve function and usability for preclinical device testing. In order to test this hypothesis, research was conducted based on two specific aims. The first aim was to develop a more ideal BVM system. The specific hypothesis in this case was that a BVM chamber could 
be created to accommodate a wider range of stent lengths and diameters, while allowing for easy graft insertion and seal-ability. The second aim was to develop next generation BVM systems, focused on future needs and technology. The hypothesis in this case was that with emerging technology the ability to test stents specifically for bifurcation lesions, as well as in long and angulated vessels, will be needed and is possible within the BVM system. The prototypes developed and the findings from constructed models are presented and discussed in the following chapters. 


\section{INITIAL DESIGN OF AN IDEAL BVM}

Introduction

A bioreactor is a system in which conditions are closely controlled in order to induce a specific behavior in living cells or tissue (Martin, Wendt and Heberer 2004; Korossis, et al. 2005). Design and construction of bioreactors are very important in tissue engineering research, because they directly provide the in vitro environment for tissue growth (Chen and Hu 2006). A bioreactor attempts to mimic physiological conditions in order to maintain and encourage cell growth. Bioreactor technologies intended for tissue engineering can be used to grow functional cells and tissues for transplantation and for controlled in vitro studies. It is the second application on which this thesis is focused: the use of bioreactors for controlled in vitro studies.

The primary objectives of tissue engineering bioreactors are to establish spatially uniform cell distributions on three dimensional scaffolds, to maintain desired concentrations of gases and nutrients in the culture medium, and to expose developing tissue to appropriate physical stimuli (Korossis, et al. 2005). The requirements of a bioreactor vary depending on the dimensions, complexity, and physiological environment of the tissue to be engineered. Most importantly, however, the bioreactor has to be able to operate over sustained periods of time under aseptic conditions so that cells are able to grow and function (Chen and $\mathrm{Hu} 2006$ ). The overall goal is to create a system that reliably and reproducibly supports the growth of functional living tissue (Martin, Wendt and Heberer 2004; Ratcliffe and Niklason 2002). 
The previously developed, original BVM system (Cardinal, et al. 2006) consists of a custom built horizontal rectangular vessel chamber with three ports: a luminal inlet, luminal outlet, and extra luminal outlet. Both outlets lead to a two-port media reservoir, with external side clamps controlling the flow path. The scaffold is attached to two barbed fittings which are inserted into the luminal inlet and luminal outlet ports located within the vessel chamber. Adjustments to length are made by pulling or pushing the tubing in or out of the chamber. The top of the chamber is optically clear allowing for easy visualization of the scaffold, media and stent placement. The chamber itself is sealed using rubber o-rings and clamped together by tightening four screws. Flow is created using a peristaltic pump, with media entering the luminal inlet and exiting through the luminal outlet and extra luminal outlet. The system can be sterilized using ethylene oxide (EO) gas. Although advantages of this original system exist, it would be desirable to improve upon this design in order to create a more ideal BVM system.

One common disadvantage among many bioreactor systems is the amount of difficulty experienced when installing or removing scaffolds (Singh, et al. 2007), and this is true for the original BVM system. This is often a challenge in itself as sterility needs to be maintained at all times. Easy handling and assembly also lowers the risk of contamination (Fisher, Mikos and Bronzino 2007). A more user friendly design is one of the primary needs of the BVM system. Easy scaffold insertion, without the need for bending or twisting, along with a method to quickly and simply seal the vessel chamber without the need for tools, would greatly improve the usability of the BVM system. 
A second need for the BVM system is the ability to accommodate a wider range of stent sizes. As discussed in detail in the introduction to this thesis, stents range in shape and size, and thus a BVM system would ideally accommodate $2.0-5.0 \mathrm{~mm}$ diameters and 8-38 mm lengths (Kandzari, Tcheng and Zidar 2002; Kraitzer, Kloog and Zilberman 2008), in order to fit the variations of coronary arteries observed in clinical practice and the stents currently available in the market.

In addition, ideally the BVM system would be easy to manufacture and utilize materials and components that are readily available and cost-effective so that scale-up is possible (Fisher, Mikos and Bronzino 2007; Martin, Wendt and Heberer 2004). Other considerations in design of the BVM system include sterilization method, incubator configuration, aseptic change of culture media, tissue monitoring, and reproducibility (Fisher, Mikos and Bronzino 2007). The aim of the work in this chapter was to improve upon the original BVM system by developing a new, more ideal design, with the hypothesis being that a simpler, easier to use and more variable design could be created and implemented for preclinical device testing. The specific design criteria to be met are summarized below:

- An overall user friendly design so that assembly is simple and straightforward

- Easy scaffold insertion and removal without the need for bending or twisting

- Easy to seal leak-proof chamber for quick and sterile assembly

- Adjustable scaffold length and diameter for greater testing variability

- Sterilizable by ethylene oxide (EO) gas

- Optically clear chamber for easy visibility of scaffold, media and stent placement 
- Easy scale-up so that multiple chambers can be configured in the incubator

- Low cost

- Easy to manufacture without the need for adhesive or special tooling

- Use of materials and components that are readily available and easy to obtain

- Use of biocompatible materials so that cells are able to grow and function 
Initial Design Models and Concepts

Initial design ideas and concepts were drawn and modeled. These designs were free flowing ideas based only on the known limitations, needs and design criteria to be met for the new BVM system. Ideas were recorded or drawn in a lab notebook over a time period of several days before any evaluations were made. Drawings were then turned into basic 3D models using SolidWorks (SolidWorks Student Design Kit 2008) for visualization and comparison. These designs were recorded without regard to feasibility of design, availability of components, cost or any other idea limiting factors. It should be noted that only the luminal inlet and luminal outlet were modeled, since location of the extra luminal outlet was not critical to the basic design. In addition, tubing attachment, connections and fittings were not addressed in these initial designs as the goal was only to develop a basic model. The five initial design ideas that were developed are modeled and described below.

Prior to the building of any prototypes the initial design ideas and concepts were reviewed and evaluated based on the following criteria: user friendly design, seal-ability, availability of materials and components, adjustability, and overall cost. Each model was examined to first determine if it could actually be built and function, and if so, whether the components were readily available or easy to create. Finally, even if the design could be built and parts were available, consideration was taken into design complexity, manufacturability and overall cost. The evaluation of each initial design is presented below following the design model and description. 


\section{Initial Design 1}

Description: The first initial design idea consisted of clear plastic rectangular top and bottom sections that meet at the center and snap together. The two sections could be separate pieces or possibly held together by a hinge. The entire midsection would contain a rubber o-ring and when snapped together the top and bottom sections would create a leak-proof seal. Two centered holes would be made on opposite ends of the box, resulting in half circle indentations on both the top and bottom sections. The two holes on either end of the box would also contain a rubber seal and when snapped together they would encompass the scaffold and tubing and create a tight leak-proof seal around the tubing. During set-up, the scaffold and tubing would be placed along the center of the bottom section, within the half circle indentations, prior to placement of the top section. Initial Design 1 is modeled in Figures 2.1 and 2.2 below.

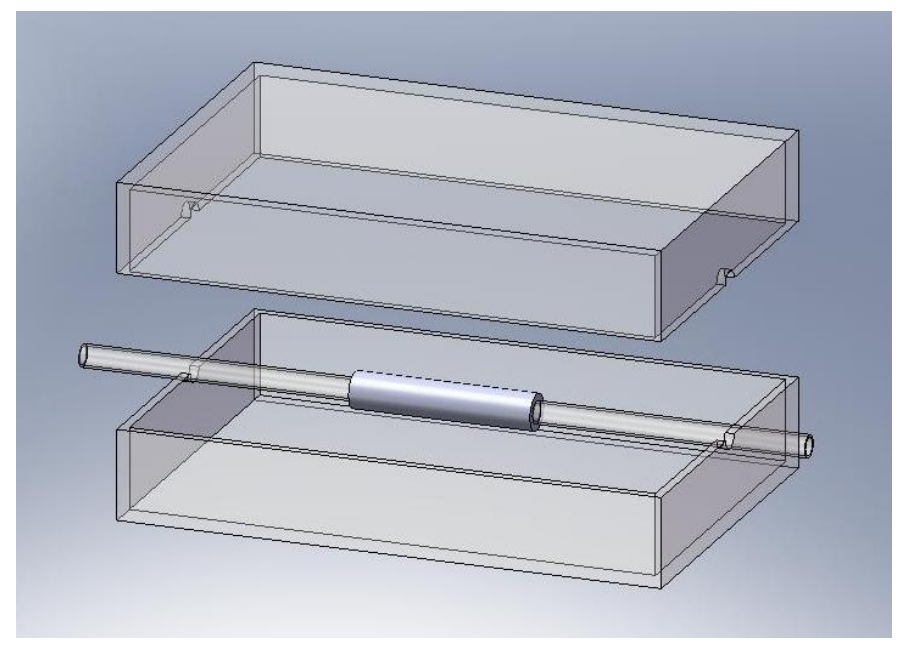

Figure 2.1- Exploded 3D model of Initial Design 1. An exploded view shows the overall design concept with the top and bottom sections separated. The scaffold and tubing is set across the bottom section and placed within the half circle indentations. 


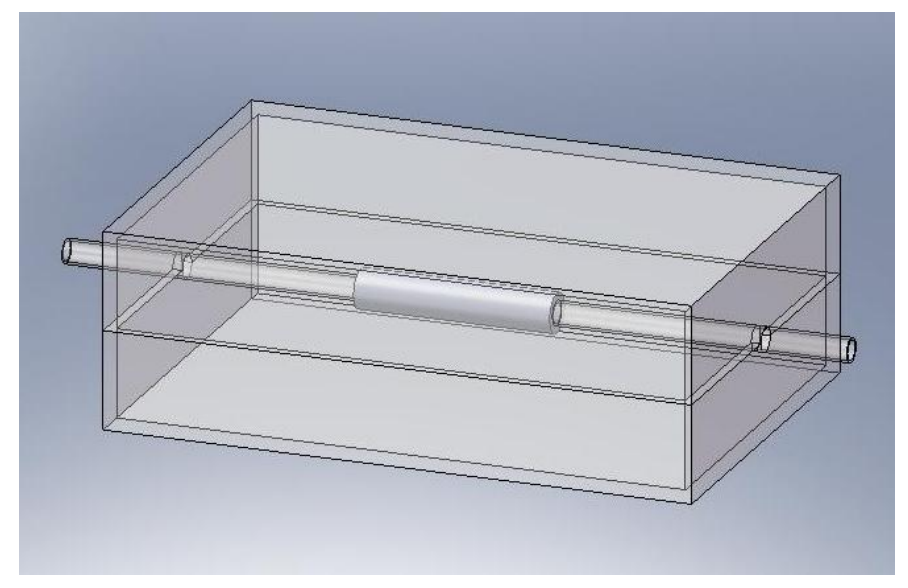

Figure 2.2- Assembled 3D model of Initial Design 1. An assembled view shows the overall design concept with the top and bottom sections snapped and sealed together. The scaffold and tubing is encompassed between the top and bottom sections, with the half circle indentations sealed around the tubing.

Evaluation: The biggest advantage of this design is that the snapping top and bottom sections would be easy to assemble and use. This design would be easy to open, close and put together. The box design would be easy to configure in an incubator and is stackable. Scaffold placement would be simple, as it would lie across the bottom section within the half circle indentations. The scaffold and tubing could be set in place without the need for stretching or bending. Clear plastic would allow for visibility and could be sterilized using ethylene oxide (EO) gas. The scaffold length would be somewhat variable, but limited by the chamber length. The limiting factor of this design is sealability. The entire midsection must seal together and the half circle indentations must create a leak-proof seal around the tubing. Manufacture of this type of seal design would be complicated, expensive and require adhesive and custom made parts. The major disadvantage of this design was that no container could be found with this configuration 
and creating a functioning seal would be challenging. The overall evaluation of Initial Design 1 is summarized below:

- User friendly design: Yes- chamber would be easy to assemble and scaffold would be easy to insert

- Seal-ability: Difficult- it would be hard to design and ensure a complete leakproof seal across the tubing and along all edges

- Availability of materials and components: Custom- some components would need to be custom built and designed

- Adjustability: Limited- longest scaffold would be limited by chamber length

- Cost: Moderate to High- due to custom parts and complex seal design 


\section{Initial Design 2}

Description: The second initial design idea consisted of two clear plastic cylindrical sections that meet at the center and slide together, encompassing the scaffold and tubing. The two sections would be separate pieces with the inside diameter of the larger section matching the outside diameter of the smaller section. The open ends of the cylindrical sections would contain rubber o-rings and when slid together the two sections would create a leak-proof seal. The resulting seal would function both in a stationary position and when the two sections are slid together or apart. Two centered holes would be made on opposite ends of the cylinder, with the tubing and scaffold placed within the center. During set-up, the scaffold would be inserted into the tubing, prior to closure of the two sections. Initial Design 2 is modeled in Figures 2.3 and 2.4 below.

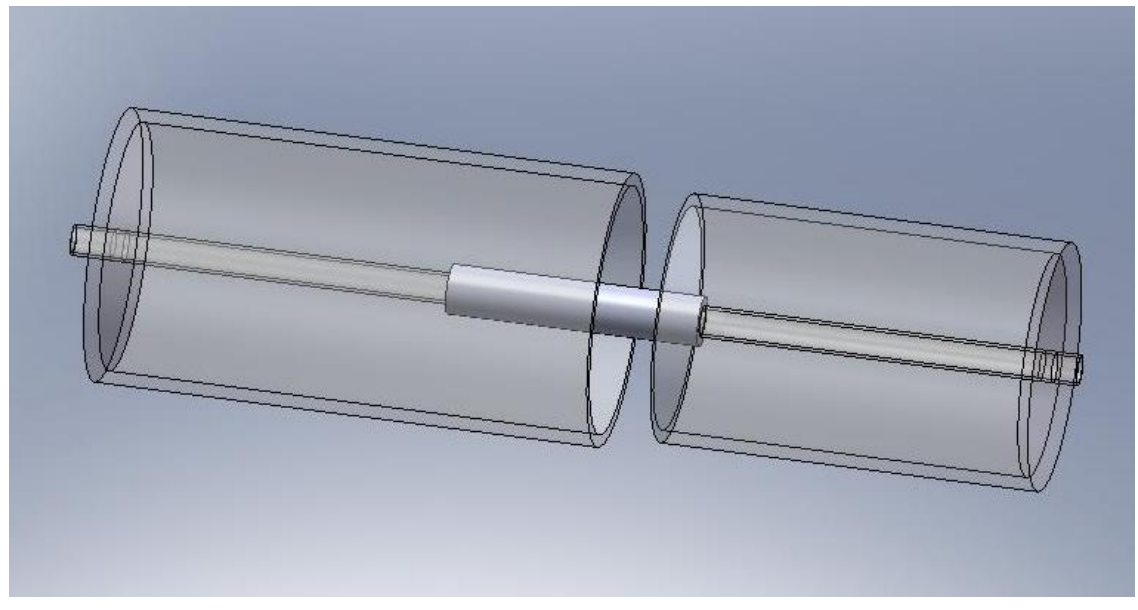

Figure 2.3- Exploded 3D model of Initial Design 2. An exploded view shows the overall design concept with the two cylindrical sections separated. The scaffold and tubing is placed within the center. 


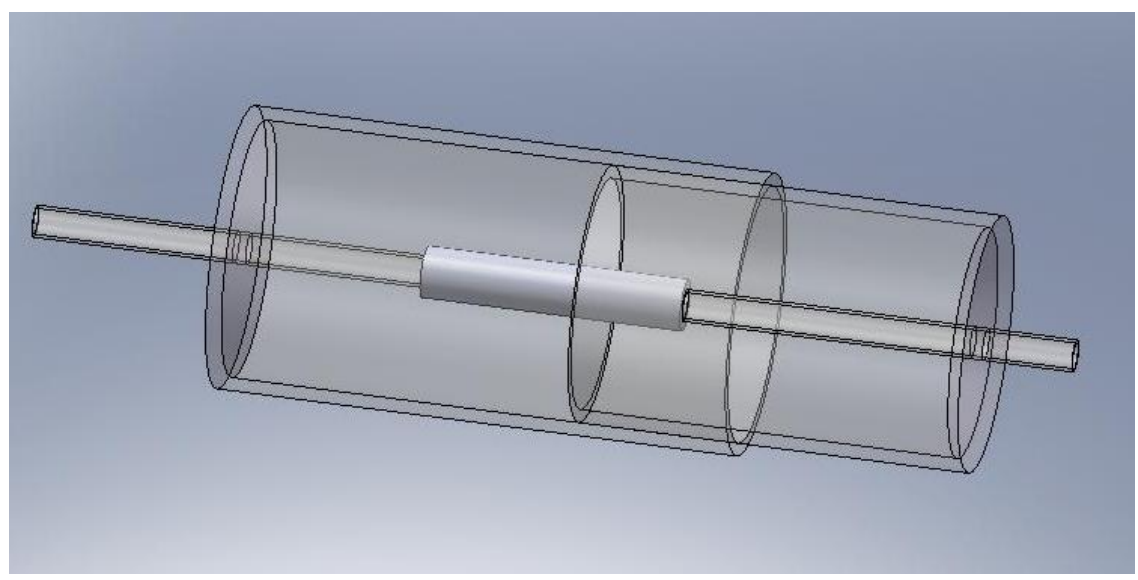

Figure 2.4- Assembled 3D model of Initial Design 2. An assembled view shows the overall design concept with the two cylindrical sections slid and sealed together. The scaffold and tubing is encompassed within the two cylindrical sections.

Evaluation: The biggest advantage of this design is that the length of the chamber would be easily adjustable. The two sliding sections would allow for variable scaffold length with precise adjustment of chamber length depending on the scaffold size used. The sliding cylindrical sections would be easy to assemble and scaffold placement would be simple. Clear plastic would allow for visibility and could be sterilized using ethylene oxide (EO) gas. Again, the overall limiting factor of this design is seal-ability. This design would need circular o-ring seals on the cylindrical sections, and the seals would need to hold and remain leak-proof while sliding. Manufacture of this type of seal design would be complicated, expensive and most likely wear out over time due to sliding. This design would be difficult to build even though the materials are available. Usability is also a concern. A cylindrical shape would be challenging to work with and place in the incubator as it would roll and is not stackable. This design would also be difficult to configure in regards to tubing adjustability, as the tubing would also need to slide and 
adjust as the chamber slides. The overall evaluation of Initial Design 2 is summarized below:

- User friendly design: Somewhat- chamber would be easy to assemble and scaffold would be easy to insert, but chamber would roll on a flat surface

- Seal-ability: Difficult- it would be hard to design and ensure a complete leakproof sliding seal that would not wear over time

- Availability of materials and components: Custom- some components would need to be custom built and designed

- Adjustability: High- chamber length can be easily adjusted to scaffold length

- Cost: Moderate to High- due to custom parts and complex seal design 
Initial Design 3

Description: The third initial design idea consisted of a clear plastic cylindrical center section with clear plastic adjustable corrugated tubing on both ends. The corrugated tubing sections could be pushed and pulled to change the overall length of the chamber. The corrugated tubing would be permanently attached and sealed to the center section with one end completely closed. The other end of the corrugated tubing would have a threaded or snapping leak-proof cap to create an opening for scaffold placement. Two centered holes would be made, one on the closed end of the cylinder and one on the cap, and the tubing and scaffold would be placed through the center. During set-up, the scaffold would be inserted into the tubing, prior to closure of the cap. Initial Design 3 is modeled in Figures 2.5 and 2.6 below.

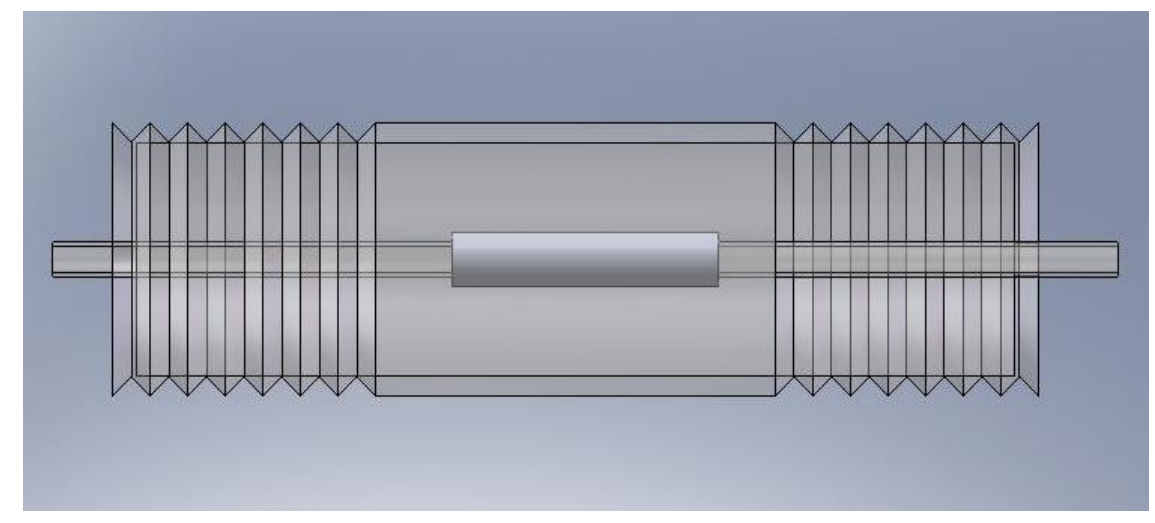

Figure 2.5- Side view 3D model of Initial Design 3. A side view shows the overall design concept with the corrugated tubing sections attached to the center section. The scaffold and tubing is placed within the center. 


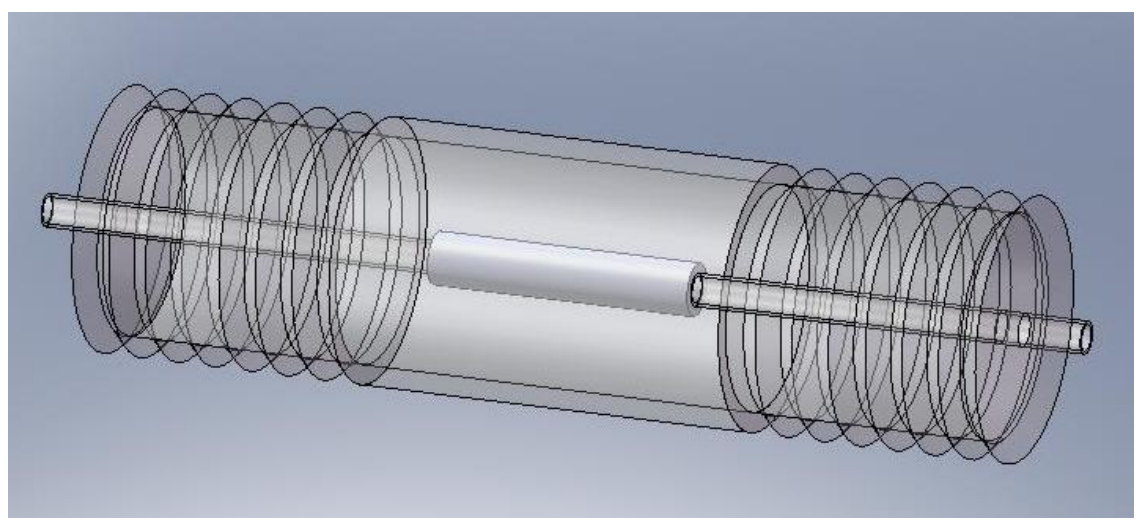

Figure 2.6- Assembled 3D model of Initial Design 3. An assembled view shows the overall design concept with the corrugated tubing sections and center sealed together. The scaffold and tubing is encompassed within the chamber.

Evaluation: The biggest advantage of this design is that the length of the chamber would be easily adjustable. The corrugated tubing could be pushed and pulled and would allow for variable scaffold length with precise adjustment of chamber length depending on the scaffold size used. The snapping cap would be easy to assemble and seal. Clear plastic would allow for visibility and could be sterilized using ethylene oxide (EO) gas. Scaffold insertion would be difficult, since access to the center can only be made from one end. Manufacture of this type of design would be difficult requiring adhesive when attaching the corrugated tubing to the center section. In addition, corrugated tubing is expensive and custom lengths and configurations would be needed. The limiting factors for this design are scaffold insertion, manufacturability and cost. Again, usability is a concern. A cylindrical shape would be challenging to work with and place in the incubator as it would roll and is not stackable. This design would be difficult to configure in regards to tubing adjustability, as the tubing would need to slide and adjust as the 
corrugated tubing is pulled or pushed. The overall evaluation of Initial Design 3 is summarized below:

- User friendly design: No- chamber would be easy to assemble but scaffold insertion would be difficult and chamber would roll when on a flat surface

- Seal-ability: Easy- entire chamber would be closed and only need a leak-proof sealing cap

- Availability of materials and components: Custom- corrugated tubing would need to be custom ordered and designed

- Adjustability: High- chamber length can be easily adjusted to scaffold length

- Cost: High- due to corrugated tubing, adhesive, manufacturing and overall complex and custom design 


\section{Initial Design 4}

Description: The fourth initial design idea consisted of a clear plastic rectangular box with snap-on sealing lid. The box and lid could be separate pieces or possibly held together by a hinge. The box would be completely open on top, allowing for complete access into the chamber, and the lid would contain a rubber o-ring. When snapped together the lid would create a leak-proof seal against the top of the box. Two centered holes would be made on opposite sides in the bottom of the box, and the tubing and scaffold would be positioned in the middle. During set-up, the scaffold and tubing would be placed in the center of the bottom section, prior to placement and closure of the lid. Initial Design 4 is modeled in Figures 2.7 and 2.8 below.

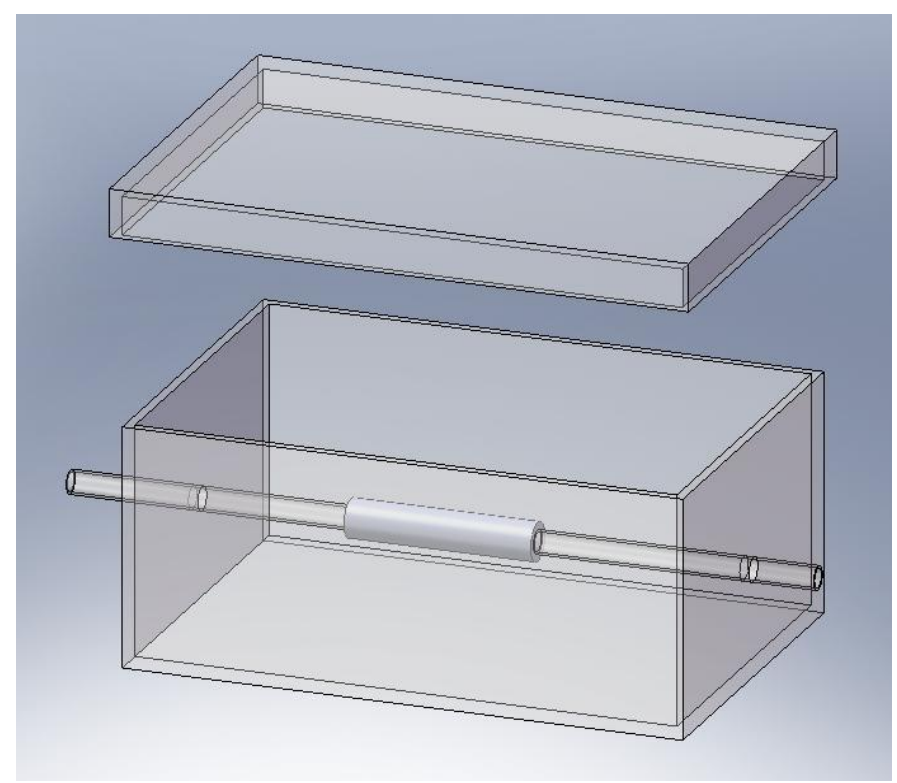

Figure 2.7- Exploded 3D model of Initial Design 4. An exploded view shows the overall design concept with the top and bottom sections separated. The scaffold and tubing is placed in the center of the bottom section. 


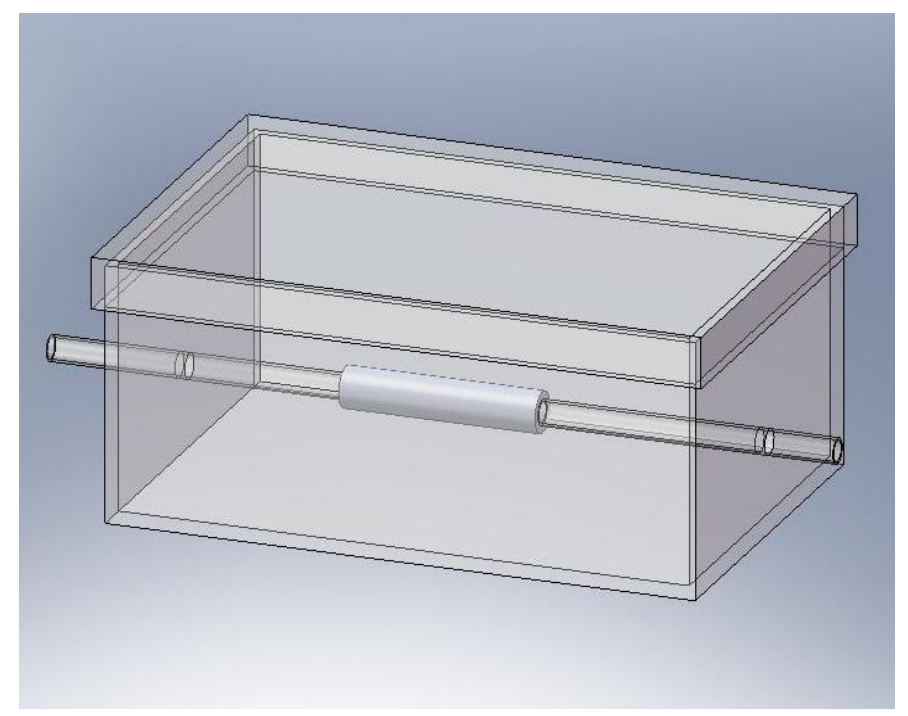

Figure 2.8- Assembled 3D model of Initial Design 4. An assembled view shows the overall design concept with the top and bottom sections snapped and sealed together. The scaffold and tubing is encompassed within the box.

Evaluation: The biggest advantage of this design is that the snapping lid would be easy to assemble, use and seal. This design would be easy to open, close and put together. The box design would be easy to configure in an incubator and is stackable. Scaffold placement would be simple, as it would be placed in the center of the bottom section. Clear plastic would allow for visibility and could be sterilized using ethylene oxide (EO) gas. Containers of this type are readily available in a number of shapes and sizes and are low in cost. No custom designed parts would be needed and manufacturing would be simple without the need for adhesive or any complex seal design. This design would be easy to build and materials are available. The scaffold length would be somewhat variable, but limited by the chamber length. The main drawback of this design is that the length of the chamber is not easily adjustable. The overall evaluation of Initial Design 4 is summarized below: 
- User friendly design: Yes- chamber would be easy to assemble and scaffold would be easy to insert

- Seal-ability: Easy- only the lid would contain a sealing o-ring which would experience minimal wear

- Availability of materials and components: Off the Shelf- standard shaped container available in many sizes with no custom parts

- Adjustability: Limited- longest scaffold would be limited by chamber length

- Cost: Low- materials and components are off the shelf and readily available with nothing custom needed 


\section{Initial Design 5}

Description: The final initial design idea consisted of a clear plastic cylindrical container with sealing snap-on lid. The cylindrical container would be open on top and the lid would contain a rubber o-ring seal. When snapped together the lid would create a leak-proof seal against the top of the cylindrical container. Two holes would be made in the lid. The tubing and scaffold would hang vertically from one of the holes, loop around in a u-shape, and exit through the second hole. Initial Design 5 is modeled in Figures 2.9 and 2.10 below.

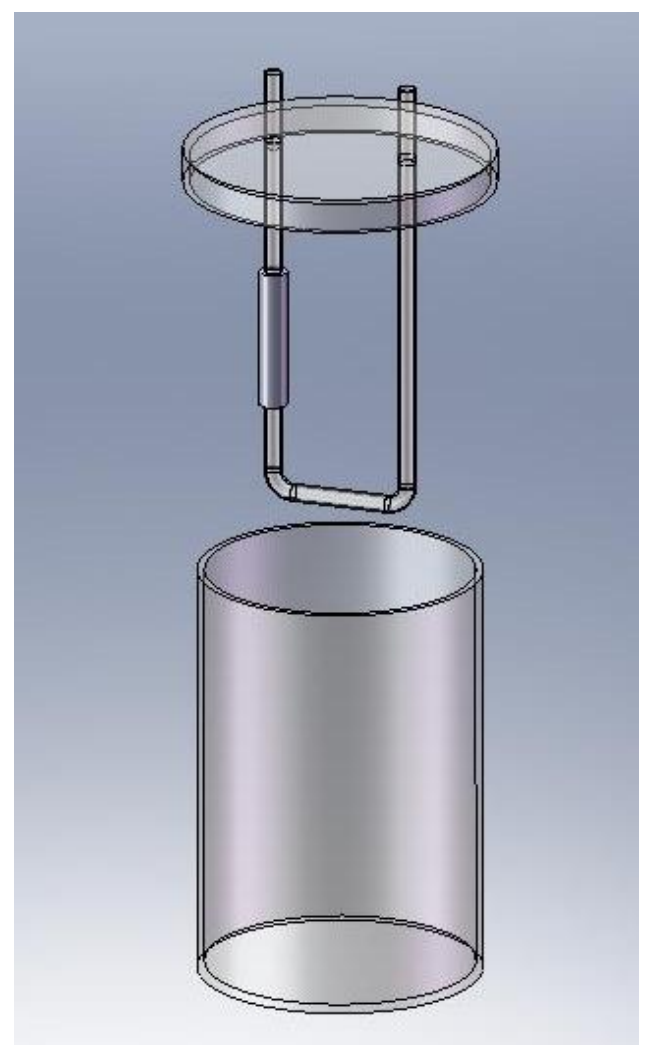

Figure 2.9- Exploded 3D model of Initial Design 5. An exploded view shows the overall design concept with the top and bottom sections separated. The scaffold and tubing are looped into a u-shape and hang vertically from the lid. 


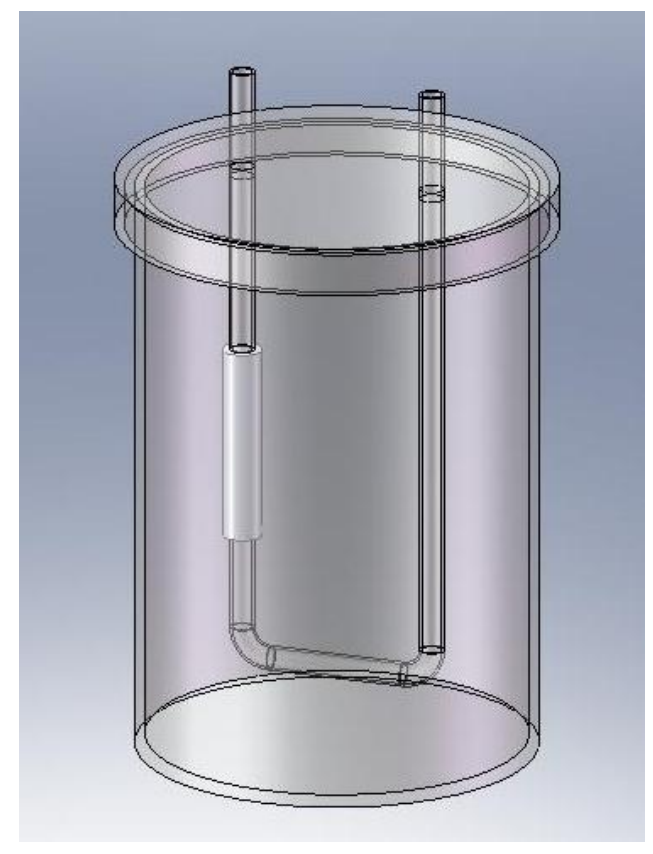

Figure 2.10- Assembled 3D model of Initial Design 5. An assembled view shows the overall design concept with the top and bottom sections snapped and sealed together. The scaffold and tubing are encompassed within the cylindrical chamber.

Evaluation: The biggest advantage of this design is that the snapping lid would be easy to assemble, use and seal. Scaffold placement would be simple, as it would hang from the lid. Clear plastic would allow for visibility and could be sterilized using ethylene oxide (EO) gas. The chamber would be easy to configure in an incubator but is not stackable due to the tubing being attached to the lid. Containers of this type are readily available in a number of shapes and sizes and are low in cost. No custom designed parts would be needed and manufacturing would be simple without the need for adhesive or any complex seal design. This design would be easy to build and materials are available. The scaffold length would be somewhat variable, but limited by the container length. In addition, the tubing length would need to be adjusted each time the scaffold length is changed. The key difference and possible challenge in this design is 
that the scaffold is oriented vertically. The overall evaluation of Initial Design 5 is summarized below:

- User friendly design: Yes- chamber would be easy to assemble and scaffold would be easy to insert

- Seal-ability: Easy- only the lid would contain a sealing o-ring which would experience minimal wear

- Availability of materials and components: Off the Shelf- standard shaped container available in many sizes with no custom parts

- Adjustability: Limited- longest scaffold would be limited by chamber length

- Cost: Low- materials and components are off the shelf and readily available with nothing custom needed 
Evaluation of Initial Design Models and Concepts

A summary of the design criteria evaluated in the previous section is given in Table 2.1 below. Initial designs 1 through 5 were evaluated based on user friendly design, seal-ability, availability of materials and components, adjustability, and overall cost. The results of these evaluated criteria were used when deciding what initial designs to move forward with and build into physical prototypes.

\begin{tabular}{|c|c|c|c|c|c|}
\hline & $\begin{array}{c}\text { Initial } \\
\text { Design 1 }\end{array}$ & $\begin{array}{c}\text { Initial } \\
\text { Design 2 }\end{array}$ & $\begin{array}{c}\text { Initial } \\
\text { Design 3 }\end{array}$ & $\begin{array}{c}\text { Initial } \\
\text { Design 4 }\end{array}$ & $\begin{array}{c}\text { Initial } \\
\text { Design 5 }\end{array}$ \\
\hline $\begin{array}{c}\text { User Friendly } \\
\text { Design }\end{array}$ & Yes & Somewhat & No & Yes & Yes \\
\hline Seal-Ability & Difficult & Difficult & Easy & Easy & Easy \\
\hline $\begin{array}{c}\text { Availability of } \\
\text { Materials and } \\
\text { Components }\end{array}$ & Custom & Custom & $\begin{array}{c}\text { Very } \\
\text { Custom }\end{array}$ & $\begin{array}{c}\text { Off the } \\
\text { Shelf }\end{array}$ & $\begin{array}{c}\text { Off the } \\
\text { Shelf }\end{array}$ \\
\hline Adjustability & Limited & High & High & Limited & Limited \\
\hline Cost & $\begin{array}{c}\text { Moderate } \\
\text { to High }\end{array}$ & $\begin{array}{c}\text { Moderate } \\
\text { to High }\end{array}$ & High & Low & Low \\
\hline
\end{tabular}

Table 2.1- Evaluation of initial design ideas. The initial design ideas were evaluated based on the following criteria: user friendly design, seal-ability, availability of materials and components, adjustability, and overall cost.

Ultimately the final decision on what designs to move forward with and build into prototypes was based on seal-ability and availability of materials and components, along with consideration of the other above stated criteria. The highest priority was choosing a design that could be easily sealed and remain leak-proof, so that sterility could be maintained. Seal-ability is extremely important since a sterile environment is needed for cell and tissue growth (Fisher, Mikos and Bronzino 2007; Chen and Hu 2006). The next 
consideration was choosing a design that could actually be built, utilizing materials and components that are readily available, since the aim of this thesis was to both design and build a BVM system for preclinical device testing. A design was needed that could be easily manufactured without the need for adhesive or custom parts. A more user friendly design was one of the primary needs of the BVM system and was the next consideration. Those designs in which scaffold placement was simple and overall usability was easy were given priority. Although cost was not a major deciding factor, the designs that utilized off the shelf components also had the advantage of being low cost. Finally, a more variable design with the ability to accommodate a wider range of stent sizes was the last consideration. In evaluating all the designs, adjustability became a minor consideration. Although a custom adjustable chamber would be nice, a longer scaffold could be easily accommodated in the other designs by simply choosing a larger container. Therefore, adjustability was given the least priority when choosing the designs with which to move forward and prototype. To summarize, the evaluated design criteria were given the following priorities:

1. Seal-ability

2. Availability of materials and components

3. User friendly design

4. Cost

5. Adjustability 
After prioritizing the above criteria and evaluating the results found in Table 2.1, only initial designs 3, 4 and 5 met the top design criteria of being easy to seal. Additionally, initial designs 4 and 5 met the second criteria utilizing materials and components that are easily available and off the shelf, rather than needing to be custom designed or ordered. Early evaluation revealed initial designs 4 and 5 as the designs with the most potential, meeting the top 4 out of 5 design criteria stated above.

While searching for available materials and components, a simple solution was found to overcome the challenge of seal-ability and manufacturability which further narrowed the overall design considerations down to initial designs 4 and 5. By using a leak-proof plastic food container with snap-on sealable lid and built in o-ring, the basic chamber design was solidified and initial designs 4 and 5 were chosen to be prototyped. Initial designs 4 and 5 also had the advantage of being both user friendly and low cost designs. Figure 2.11 below shows commercially available plastic food containers with snap-on lids in several different shapes and sizes.

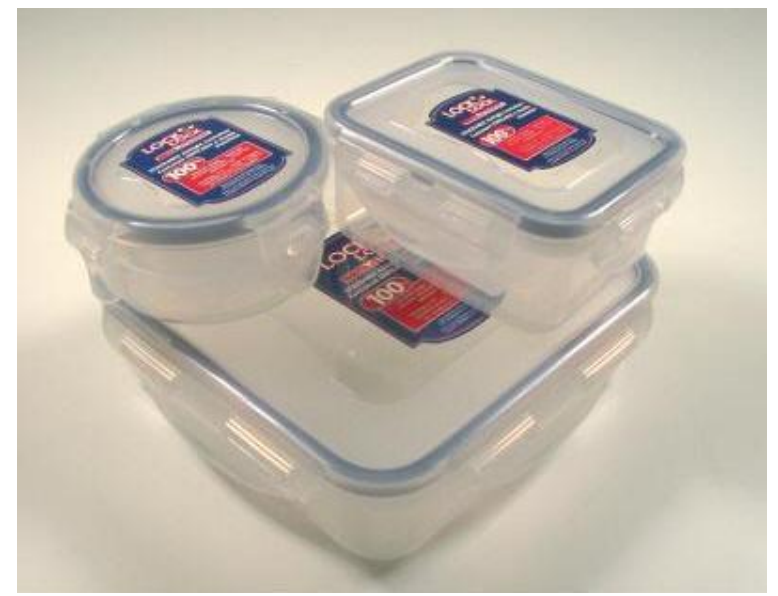

Figure 2.11- Leak-proof plastic food container with snap-on sealing lid. 
A leak-proof plastic food container was chosen for the chamber design because it is easy to seal, made of clear biocompatible and sterilizable plastic, low cost and available in local stores. Leak-proof polypropylene food containers come in a variety of shapes, sizes and configurations with lids that have built-in silicone o-rings. The leakproof food container meets the following design criteria stated in the introduction:

$\checkmark$ An overall user friendly design so that assembly is simple and straightforward

- Standard container configuration with snap-on lid

$\checkmark$ Easy to seal leak-proof chamber for quick and sterile assembly

- Lid has built-in silicone o-ring and latches that snap to seal

$\checkmark$ Sterilizable by ethylene oxide (EO) gas

- Polypropylene and silicone can withstand EO sterilization

$\checkmark$ Optically clear chamber for easy visibility of scaffold, media and stent placement

- All sides of container are see-through

$\checkmark$ Easy scale-up so that multiple chambers can be configured in the incubator

- Containers are stackable and modular

$\checkmark$ Low cost

- Approximately \$3-10 per container, depending on size

$\checkmark$ Made of materials and components that are readily available and easy to obtain

- Containers can be purchased in local stores or online

$\checkmark$ Made of biocompatible materials so that cells are able to grow and function

- Polypropylene and silicone are biocompatible materials 


\section{Design Prototypes}

After identifying containers and identifying initial designs 4 and 5 as the best potential candidates, the next step was to build physical prototypes to evaluate and further narrow the selection to one final design. The following two prototypes were constructed by purchasing leak-proof plastic food containers from Linens-N-Things, fittings and connections from Value Plastics, Qosina, and McMaster-Carr and flexible tubing from Cole-Parmer. The two prototypes were assembled based on the above models of initial designs 4 and 5 and are shown and described below. Details on how the fittings were secured, specific parts used and the entire assembly process will be addressed in the following chapter after the final design has been chosen. The goal of this section was to build physical prototypes that could be held, compared, and evaluated by potential users in order to determine the final design.

\section{Design Prototype 1}

Description: The first prototype built was based on the model of initial design 4 . A rectangular, clear, plastic, leak-proof food container with snap-on sealing lid was used for the chamber. The container used measured approximately 5 " long x 3.5 " wide x 2" deep. The lid contained a silicone o-ring seal and four snapping latches that locked to the bottom section securing the bottom and lid together. Two holes were made on opposite sides of the container and threaded luer lock to barbed connection fittings were secured within the holes. The scaffold was horizontally positioned between the fittings using the appropriate connections. Adjustment to scaffold length could be made by adding or 
removing fittings. Photographs of the physical prototype built are shown below in

Figures 2.12 and 2.13.

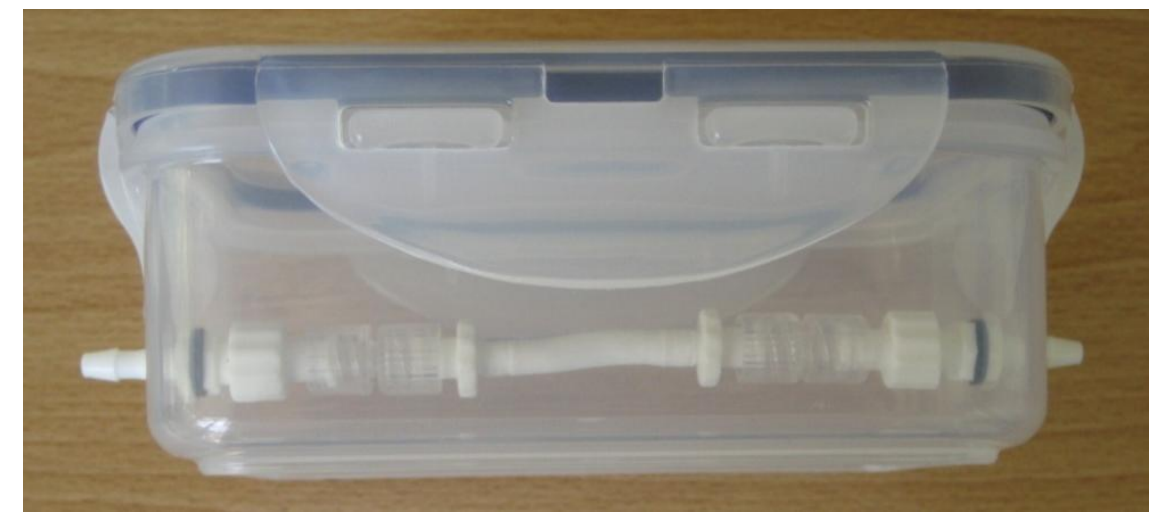

Figure 2.12- Side view of Prototype 1. A rectangular plastic leak-proof container with snap-on sealing lid was used for the chamber.

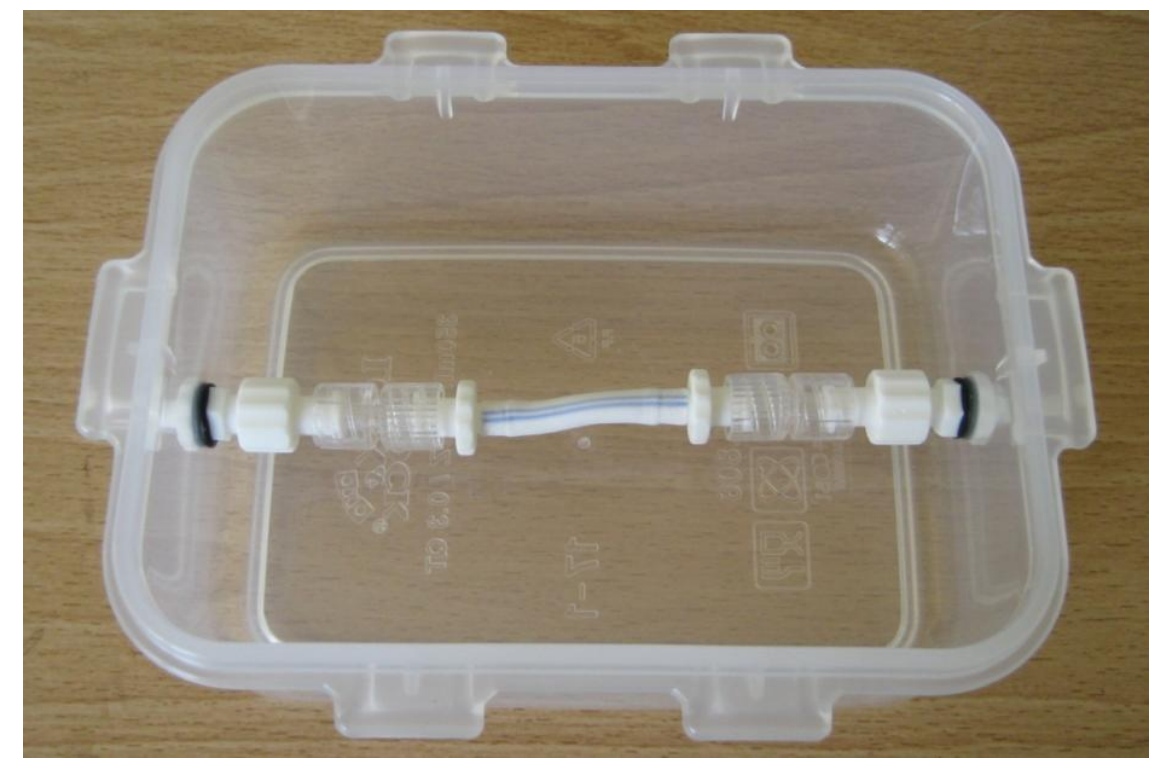

Figure 2.13- Top view of Prototype 1. The scaffold was horizontally positioned within the chamber using the appropriate connections. 


\section{Design Prototype 2}

Description: The second prototype built was based on the model of initial design 5. A tall rectangular, clear, plastic, leak-proof food container with snap-on sealing lid was used. The container used measured approximately 4" wide x 4" wide x 4.5 " tall. The lid contained a silicone o-ring seal and four snapping latches that locked to the bottom section securing the bottom and lid together. Two holes were made in the lid of the container and threaded luer lock to barbed connection fittings were secured within the holes. The scaffold was hung vertically from the lid using the appropriate connections and the tubing was configured in a u-shape. Adjustment to scaffold length could be made by changing the opposite side tubing length. A photograph of the physical prototype built is shown below in Figure 2.14.

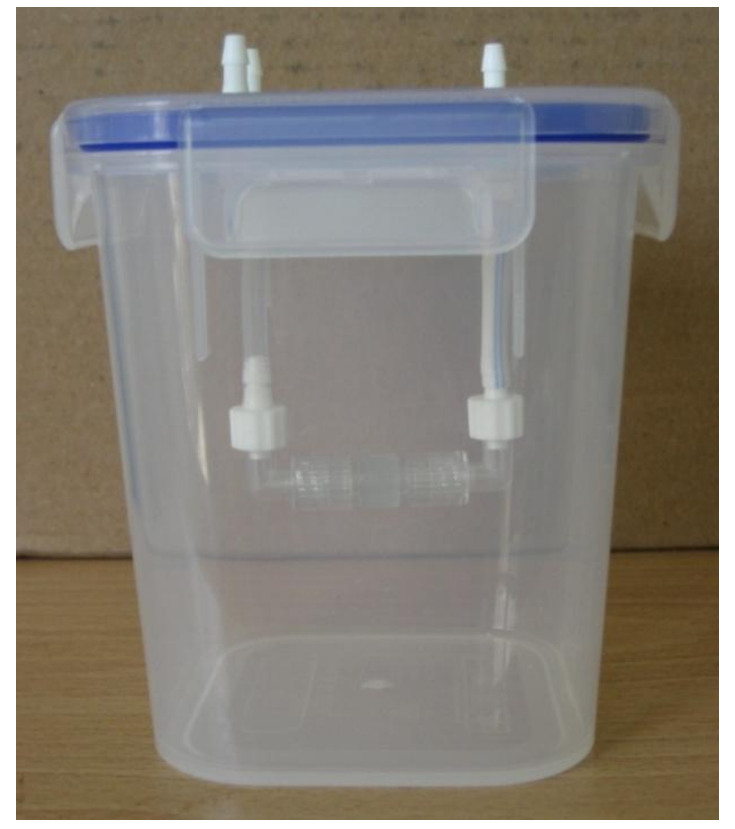

Figure 2.14- Front view of Prototype 2. A rectangular plastic leak-proof container with snap-on sealing lid was used for the chamber. The scaffold hung vertically from the lid. 


\section{Evaluation of Design Prototypes}

Prototypes 1 and 2 were evaluated in comparison with the original BVM system and to each other based on an expanded list of key criteria. The original BVM system along with physical Prototypes 1 and 2 were handled and used by several individuals in order to evaluate overall usability and ease of scaffold insertion. Measurable criteria evaluated included volume, maximum scaffold length and diameter, and maximum stent size. Observable criteria included graft orientation, closure mechanism, need for assembly tools, and stack-ability. Other criteria included cost and chamber design. Table 2.2 below summarizes the results of the compared criteria of the original BVM system, Prototype 1 and Prototype 2.

\begin{tabular}{|c|c|c|c|}
\hline & $\begin{array}{c}\text { Original BVM } \\
\text { System }\end{array}$ & $\begin{array}{c}\text { Design } \\
\text { Prototype 1 }\end{array}$ & $\begin{array}{c}\text { Design } \\
\text { Prototype 2 }\end{array}$ \\
\hline Overall Usability & moderate & easy & easy \\
\hline Scaffold Insertion & difficult & easy & moderate \\
\hline Chamber Volume & $25 \mathrm{ml}$ & $350 \mathrm{ml}$ & $700 \mathrm{ml}$ \\
\hline Graft Orientation & horizontal & horizontal & vertical \\
\hline Closure Mechanism & screws & locking snaps & locking snaps \\
\hline Assembly tools required & yes & no & no \\
\hline Stackable & no & yes & no \\
\hline Max Scaffold Length & $4.5 \mathrm{~cm}$ & $5.5 \mathrm{~cm}$ & $6 \mathrm{~cm}$ \\
\hline Scaffold Diameters & $3-4 \mathrm{~mm}$ & $2-6 \mathrm{~mm}$ & $2-6 \mathrm{~mm}$ \\
\hline Maximum Stent Length & $25 \mathrm{~mm}$ & $35 \mathrm{~mm}$ & $40 \mathrm{~mm}$ \\
\hline Stent Diameters & $3-4 \mathrm{~mm}$ & $2-6 \mathrm{~mm}$ & $2-6 \mathrm{~mm}$ \\
\hline Cost & moderate & low & low \\
\hline Chamber Design & custom & off the shelf & off the shelf \\
\hline
\end{tabular}

Table 2.2- Evaluation of design prototypes versus original BVM system. The design prototypes were compared to the original BVM system and evaluated based on objective, measurable and observable criteria. 
Prototype 1 and Prototype 2 met all design criteria stated in the introduction. When compared to the original BVM system, both prototypes also had the advantage of being more user friendly with easier scaffold placement and utilized a simpler closure mechanism without the need for assembly tools. The overall usability and ease of scaffold insertion was evaluated by the individuals that make up the 2008 BVM Lab Group, after handling and using all three designs. It was observed that locking snaps provided a quick and simple sealing method when compared with the previous method of tightening screws. Also, by using rotating fittings in the prototype designs the need to bend, push or twist the scaffold during insertion was eliminated.

In addition to usability characteristics, the prototypes were lower cost and chamber designs were non-custom and off the shelf. Maximum scaffold length and diameter was also greater in the prototype designs, resulting in the accommodation of a wider range of stent sizes. The stent sizes that could be inserted into the scaffold were estimated by taking the maximum scaffold length and subtracting $2 \mathrm{~cm}$ to take into account the amount of material used for attachment and the amount of non-useable area.

As described above, Prototypes 1 and 2 have many advantages over the original BVM system. The next step in designing a new, more ideal BVM was to compare Prototypes 1 and 2 to each other. The significant difference in design between Prototype 1 and Prototype 2 was scaffold orientation. The original BVM system utilizes a horizontal scaffold orientation and previous evaluation and use of a vertically oriented scaffold have revealed difficulties in cell sodding, with the cells tending to migrate to the bottom of the scaffold due to gravity (KO Cardinal, unpublished data). Therefore the 
biggest disadvantage of Prototype 2 was the vertical scaffold orientation. Chamber volume was also compared and considered. Although not critical, a smaller volume is more advantageous since it requires less media to fill. Both prototypes were much larger in volume than the original BVM system, however, Prototype 2 was twice the volume of Prototype 1. In addition to a horizontal scaffold orientation and smaller chamber volume, Prototype 1 also has the advantage of being stackable when compared to Prototype 2. As a result of these advantages, Prototype 1 was chosen to be used for the final design.

\section{Final Design}

Based upon the previously discussed evaluation, Prototype 1 was chosen to be used as the final design. The following chapter will review in detail the design, construction and set-up of this Ideal BVM system as well as the testing performed to validate and verify its functionality and use. 


\section{FINAL DESIGN OF IDEAL BVM}

Introduction

As stated in the previous chapter, design and construction of bioreactors are important in tissue engineering research because they directly provide the in vitro environment for tissue growth (Chen and $\mathrm{Hu}$ 2006). A bioreactor must operate over sustained periods of time under aseptic conditions so that cells are able to grow and function. The goal is to create a system that reliably and reproducibly supports the growth of functional living tissue (Martin, Wendt and Heberer 2004; Ratcliffe and Niklason 2002). In the case of a Blood Vessel Mimic (BVM), the goal is to create an environment to cultivate tissue engineered blood vessels (TEBV) in order to serve as a living vessel model for preclinical device testing (Cardinal, et al. 2006). The aim of the work in the previous chapter was to improve upon the original BVM system by developing a new, more ideal design. A simpler, easier to use and more variable design was selected from several potential ideas and an initial physical prototype was built and modeled.

The new Ideal BVM system developed and prototyped in the previous chapter consists of an off the shelf leak-proof plastic horizontal rectangular vessel chamber with a luminal inlet, luminal outlet and extra luminal outlet. The outlets lead to a two-port media reservoir, with stopcocks controlling the flow path. The scaffold is attached to two rotating luer fittings which are attached to the luminal inlet and luminal outlet ports located within the vessel chamber. Adjustments to length are made by adding or removing luer fittings. All sides of the chamber are optically clear allowing for easy visualization of the scaffold, media and stent placement. The chamber itself is sealed by a 
built-in silicone o-ring and clamped together by closing four snapping latches. The system can be sterilized using ethylene oxide (EO) gas. The specific design criteria met by the Ideal BVM were summarized in Chapter 2 and provided the motivation for moving forward with this design. Therefore, the aim of the work in this chapter is to describe in detail the finalized design, construction and set-up of this Ideal BVM system and the testing performed to validate and verify its functionality and use. 
Materials and Methods

Ideal BVM System

This section outlines the steps taken to build the new Ideal BVM system. The assembly process is described in detail and includes the specific parts used along with detailed illustrations. While this section provides the materials and methods for construction of the Ideal BVM system, a detailed list of parts and drawings, as well as complete step-by-step directions can also be found in Appendix B and C respectively. The new, Ideal BVM system was constructed as follows.

To begin, the luminal inlet, luminal outlet and extra luminal outlet were created and assembled. Three 15/64" holes were drilled into the sides of a $350 \mathrm{ml}, 5 "$ x 4" x 2", semitransparent, polypropylene rectangular snap and seal leak-proof food container (Lock \& Lock P/N HPL806). Two holes were drilled on opposite sides, one in the center of each face, directly across from one another. The centered hole on the left hand side became the luminal inlet and the centered hole on the right hand side became the luminal outlet. A third hole was drilled adjacent to the centered face hole on the right hand side of the container. This hole became the extra luminal outlet. Using a razor blade, the edges of the holes were cleaned of any plastic debris that resulted from the use of the drill. A soft black Buna-N o-ring, AS568A dash number 006, durometer 50A, 1/4" OD, 1/8" ID, 1/16" W (McMaster-Carr P/N 2418T112) was slid onto a white nylon, female luer lug style panel mount connector, 1/4-28 UNF to 200 series barb, 1/8" ID tubing (Value Plastics P/N FTLB230-1) and threaded through one of the drilled holes with the barbed portion exiting the container. A second o-ring was placed over the barbed section and a 
white nylon, 1/4-28 UNF panel mount lock nut (Value Plastics P/N LNS-1) was screwed on tightly, pressing the o-rings against the inside and outside of the container walls, creating a leak-proof seal. Once securely tightened, a drop of Loctite 4011 medical grade adhesive (McMaster-Carr P/N 1818A4) was placed between the lock nut and the threaded portion of the barbed fitting to secure it in place and prevent loosening. This procedure was repeated for the remaining drilled holes. Figures 3.1 and 3.2 below illustrate this assembly procedure.

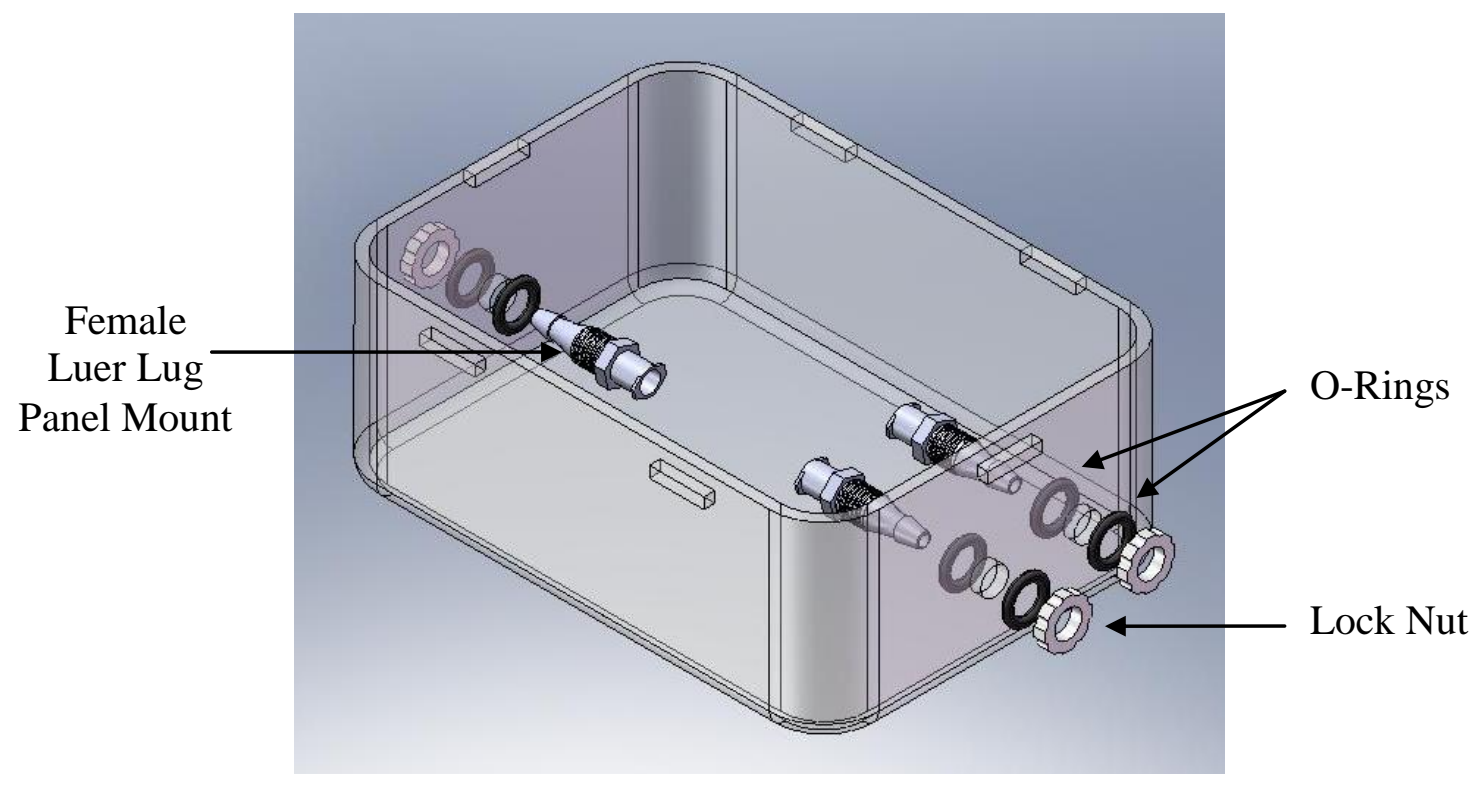

Figure 3.1- Assembly of the luminal inlet and luminal outlets. Each of the three 15/64" holes was assembled using two o-rings, a female luer lug panel mount and a lock nut. 


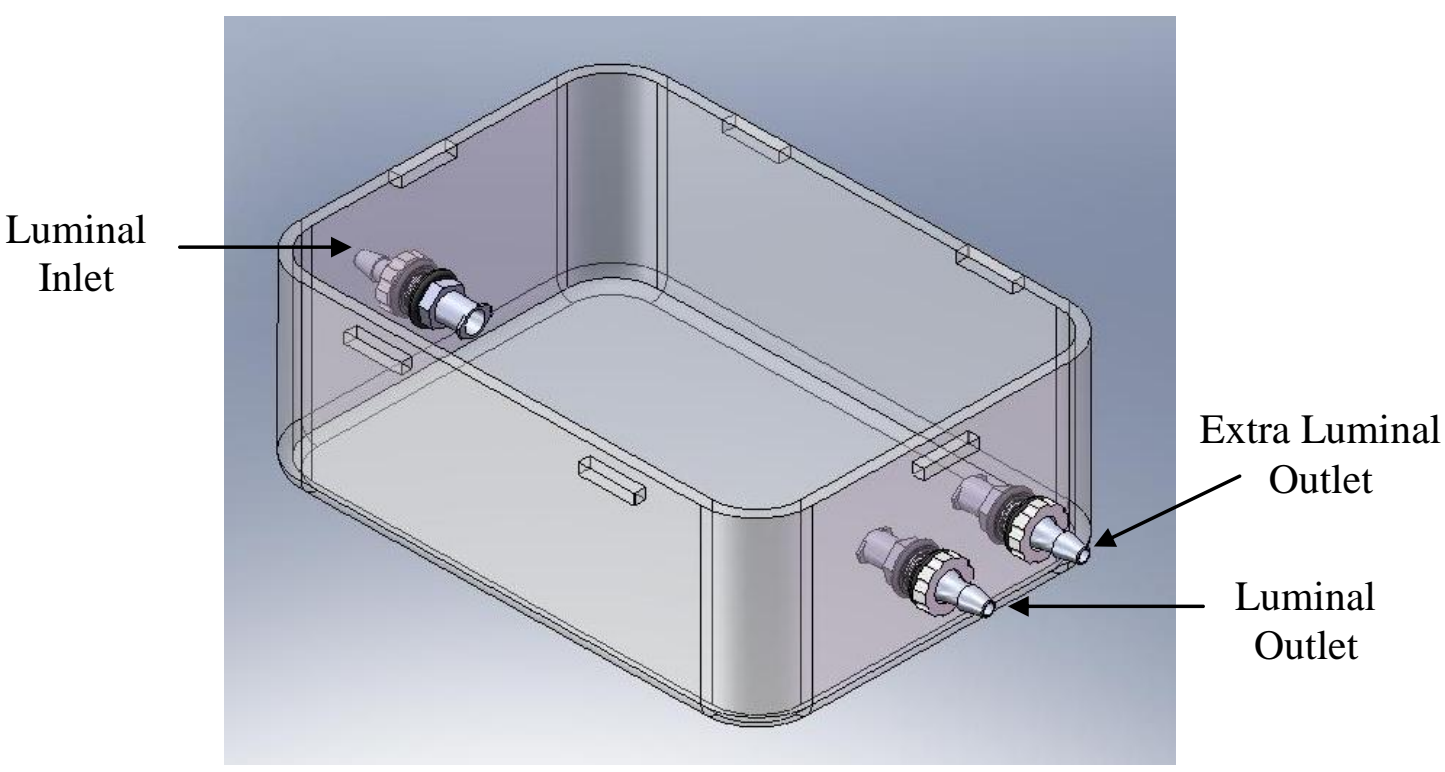

Figure 3.2- Completed assembly of the luminal inlet and luminal outlets. The female luer lug panel mounts were threaded through the holes drilled in the plastic container and the lock nuts were tightened, pressing the o-rings against the container walls creating a seal.

To complete the luminal inlet assembly a 2" piece of Tygon® silicone tubing, 1/8" ID x 1/4" OD (Cole-Parmer P/N WU-95702-06) was inserted onto the barbed fitting of the luminal inlet exiting the left side of the container. A white nylon, female luer thread style to 200 series barb, 1/8" ID tubing (Value Plastics P/N FTLL230-1) was attached to the open end of the silicone tubing attached to the luminal inlet. Finally, a three-way polycarbonate stopcock with two female and one male luer lock ends (Value Plastics P/N VPB1000079N) was attached to the female luer end on the luminal inlet tubing. The assembly process described is illustrated in Figures 3.3 and 3.4 below. 


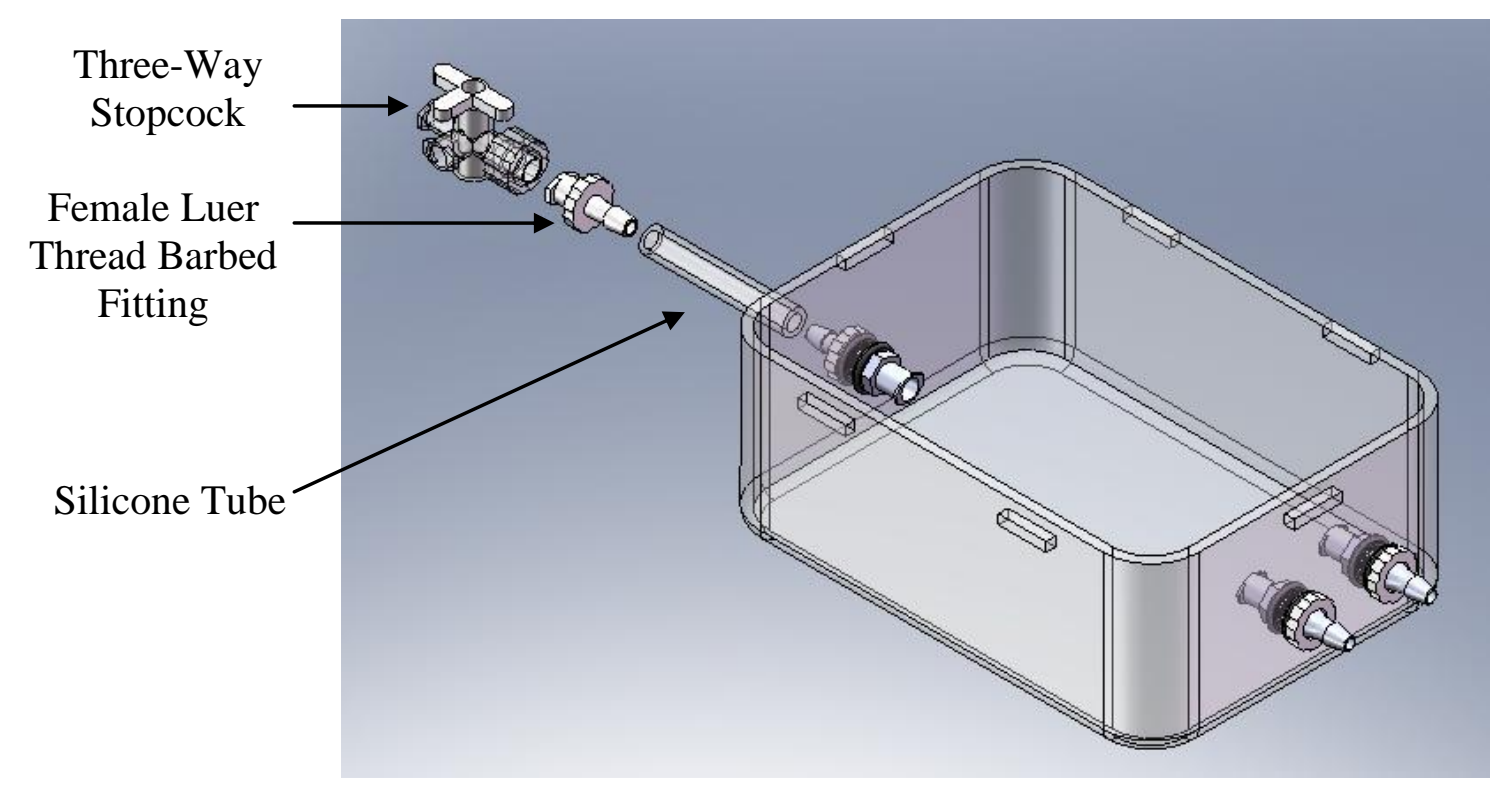

Figure 3.3- Luminal inlet tubing assembly. The luminal inlet consists of a short piece of silicone tubing, a female luer thread barbed fitting, and a three-way stopcock.

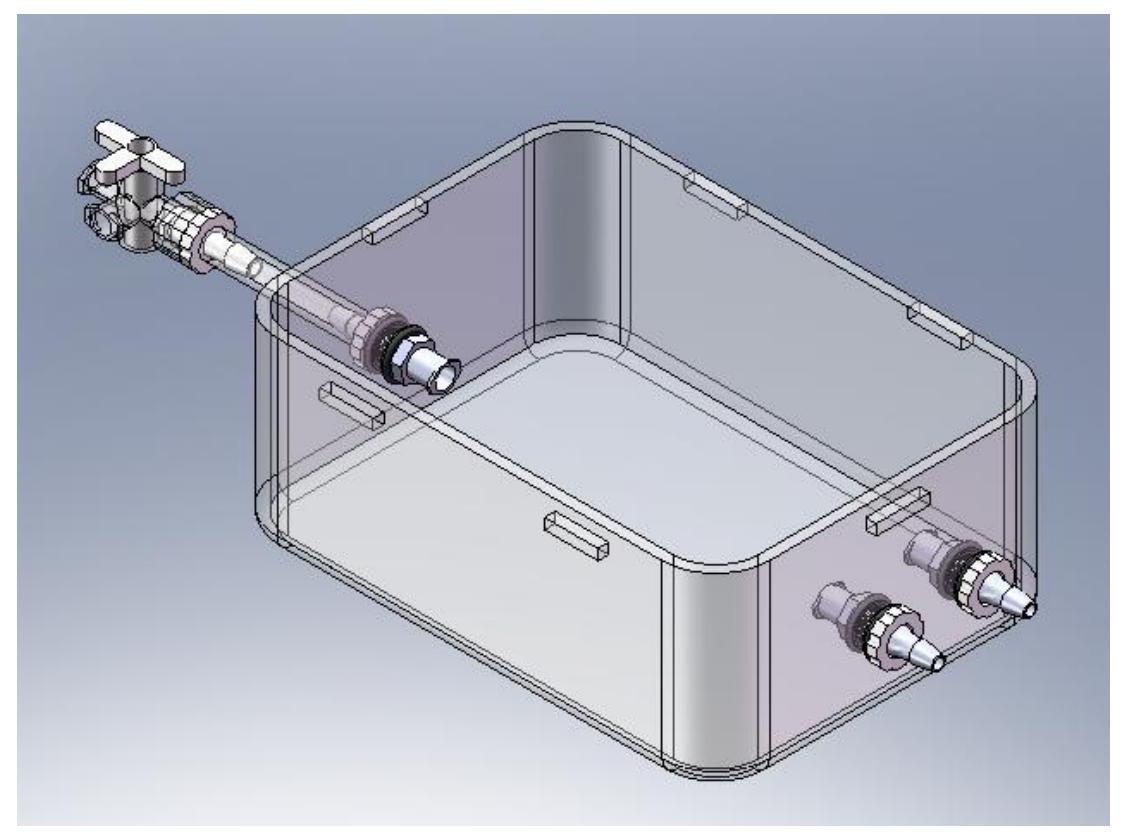

Figure 3.4- Completed luminal inlet tubing assembly. 
To complete the luminal outlet and extra luminal outlet assembly a 1 " piece of Tygon ${ }^{\circledR}$ silicone tubing, 1/8" ID x 1/4" OD (Cole-Parmer P/N WU-95702-06) was inserted onto the barbed fitting of the luminal outlet and a 5" piece of the same silicone tubing was inserted onto the barbed fitting of the extra luminal outlet, both exiting the right side of the container. A white nylon, male luer integral lock ring to 200 series barb, 1/8" ID tubing (Value Plastics P/N MTLL230-1) was attached to the open end of the silicone tubing attached to the luminal outlet. A one-way polycarbonate stopcock with one female and one male luer lock end (Value Plastics P/N VP455980) was attached to the male luer end on the luminal outlet tubing. A white nylon, female luer thread style to 200 series barb, 1/8" ID tubing (Value Plastics P/N FTLL230-1) was attached to the male end of the one-way stopcock, and a 2" piece of Tygon® silicone tubing, 1/8" ID x 1/4" OD (Cole-Parmer P/N WU-95702-06) was inserted onto the barbed fitting of the female luer attached to the one-way stopcock.

Next, the open ends of the silicone tubing attached to the luminal outlet and extra luminal outlet were attached to a white nylon, Y-connector with 200 series barbs, 1/8" ID tubing (Value Plastics P/N Y230-1). The attachment of the luminal outlet and extra luminal outlet tubing to the Y-connector created one main outlet. Finally, a 1" piece of Tygon® silicone tubing, 1/8" ID x 1/4" OD (Cole-Parmer P/N WU-95702-06) was inserted onto the barbed fitting of the Y-connector and a white nylon, male luer integral lock ring to 200 series barb, 1/8" ID tubing (Value Plastics P/N MTLL230-1) was inserted into the open end of the silicone tubing attached to the Y-connector. A one-way polycarbonate stopcock with one female and one male luer lock end (Value Plastics P/N VP455980) was attached to the male luer end on the Y-connector tubing. Figure 3.5 
provides an overall view of the assembly process, and Figures 3.6 and 3.7 provide a closer view of the luminal outlet and extra luminal outlet assembly.

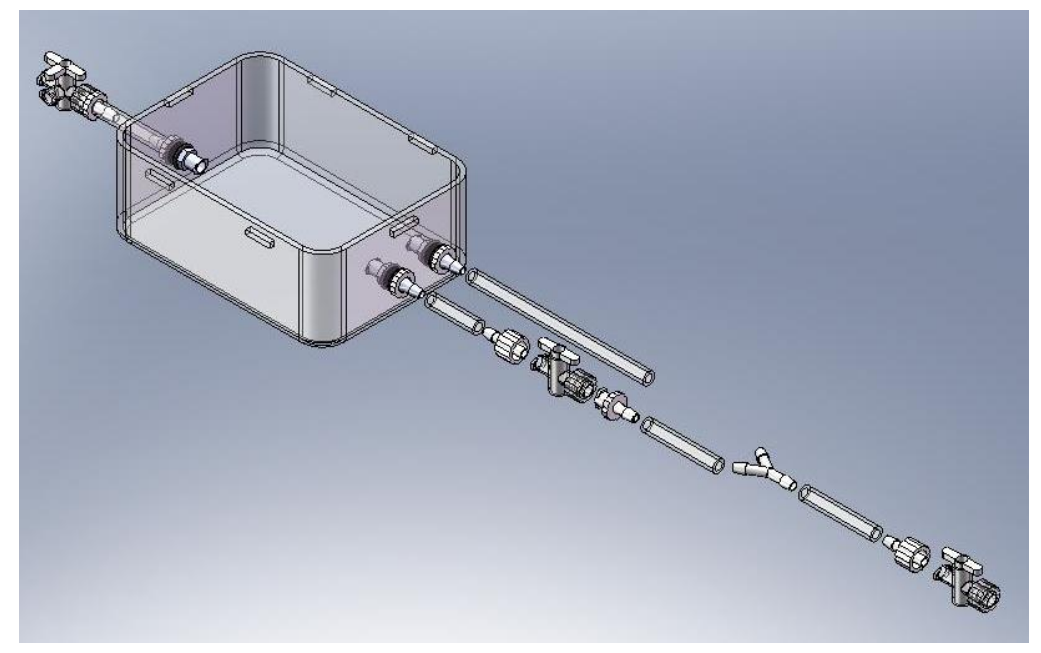

Figure 3.5- Overall view of luminal outlet and extra luminal outlet assembly.

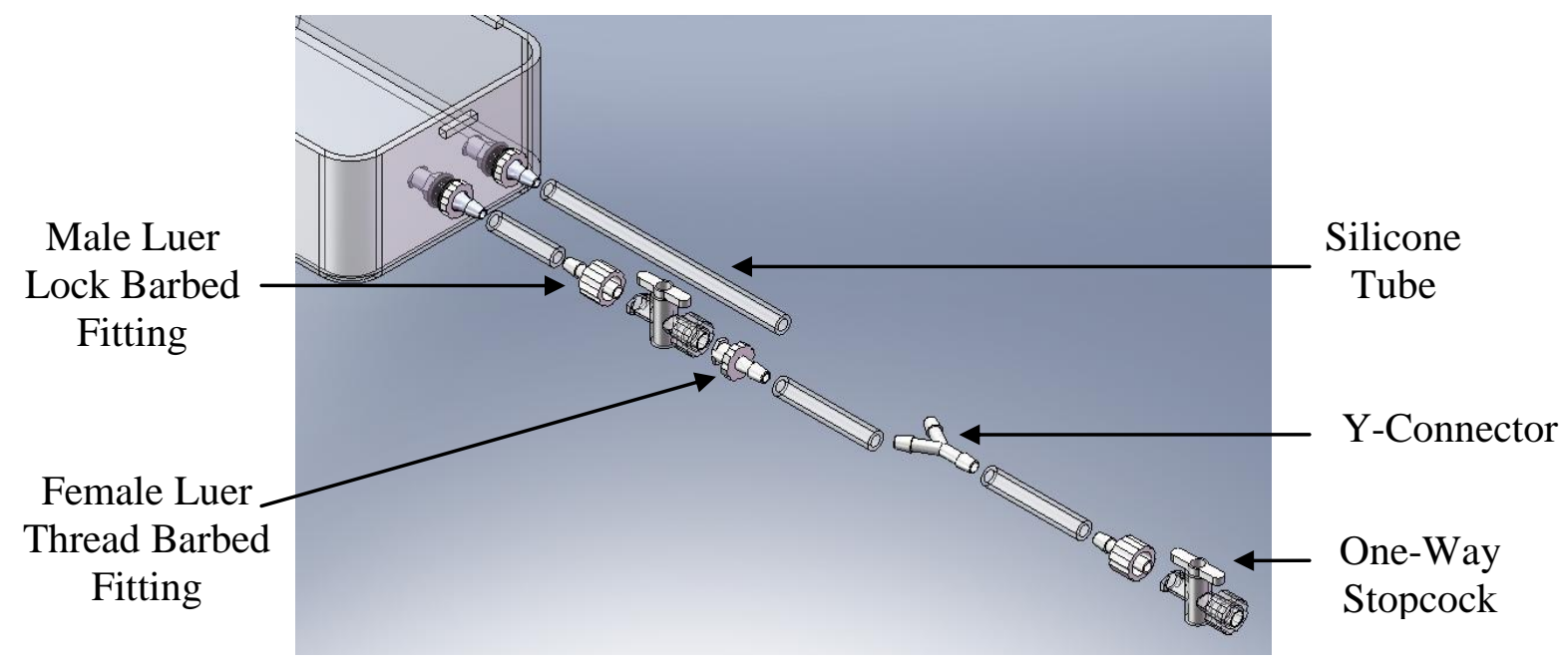

Figure 3.6- Detailed view of luminal outlet and extra luminal outlet assembly. The luminal outlet assembly consists of a one-way stopcock, while the extra luminal outlet is a simple tube. The luminal outlet and extra luminal outlet are both attached to a Yconnector and meet to form one outlet ending with a one-way stopcock. 


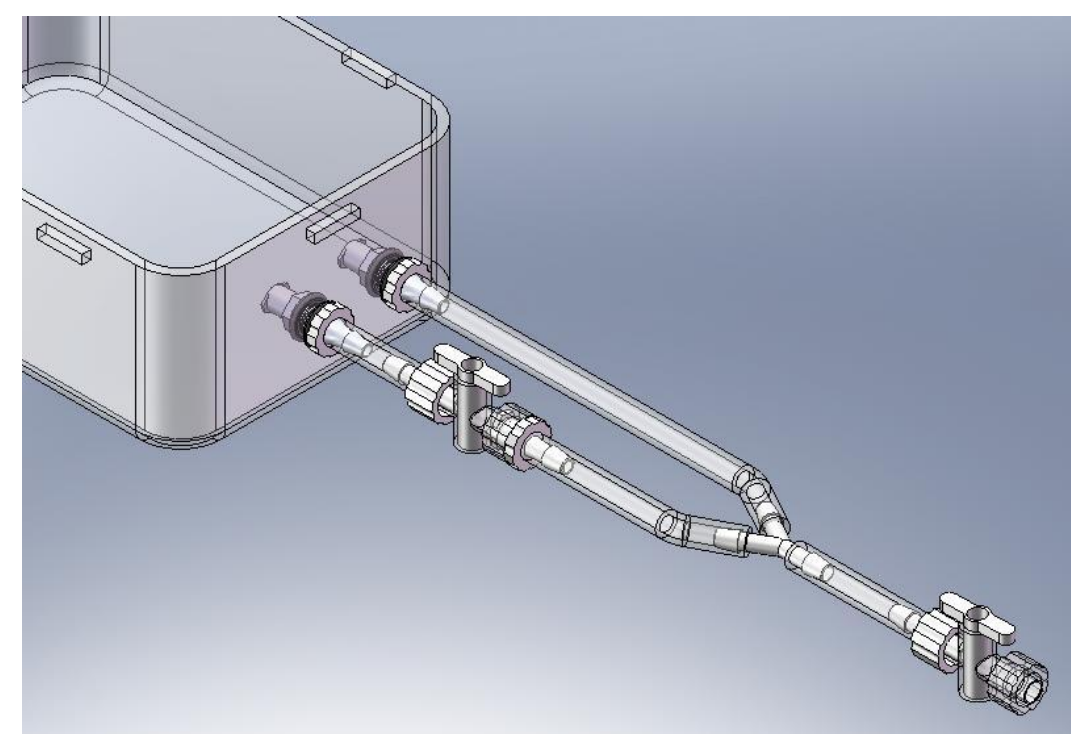

Figure 3.7-Completed luminal outlet and extra luminal outlet assembly. The two outlets meet at the Y-connector combining to form one main outlet.

To complete the inside of the chamber, two male-to-male luer lock connectors, with one rotating collar (Qosina P/N 17644), were attached to the female luer lock connector ends of the luminal inlet and luminal outlet, extending into the chamber. The male-to-male connectors were attached so that the rotating collars extended into the chamber and were free to rotate. Figures 3.8 and 3.9 below illustrate this assembly procedure. To shorten the distance between the two ends of the male-to-male luer lock connectors, white nylon male luer integral lock ring to female luer thread style couplers (Value Plastics P/N LC34-1) can be attached prior to the attachment of the male-to-male luer lock connectors. The attachment of one coupler to both the luminal inlet and luminal outlet is illustrated in Figures 3.10 and 3.11 below and will be detailed in a later section. 


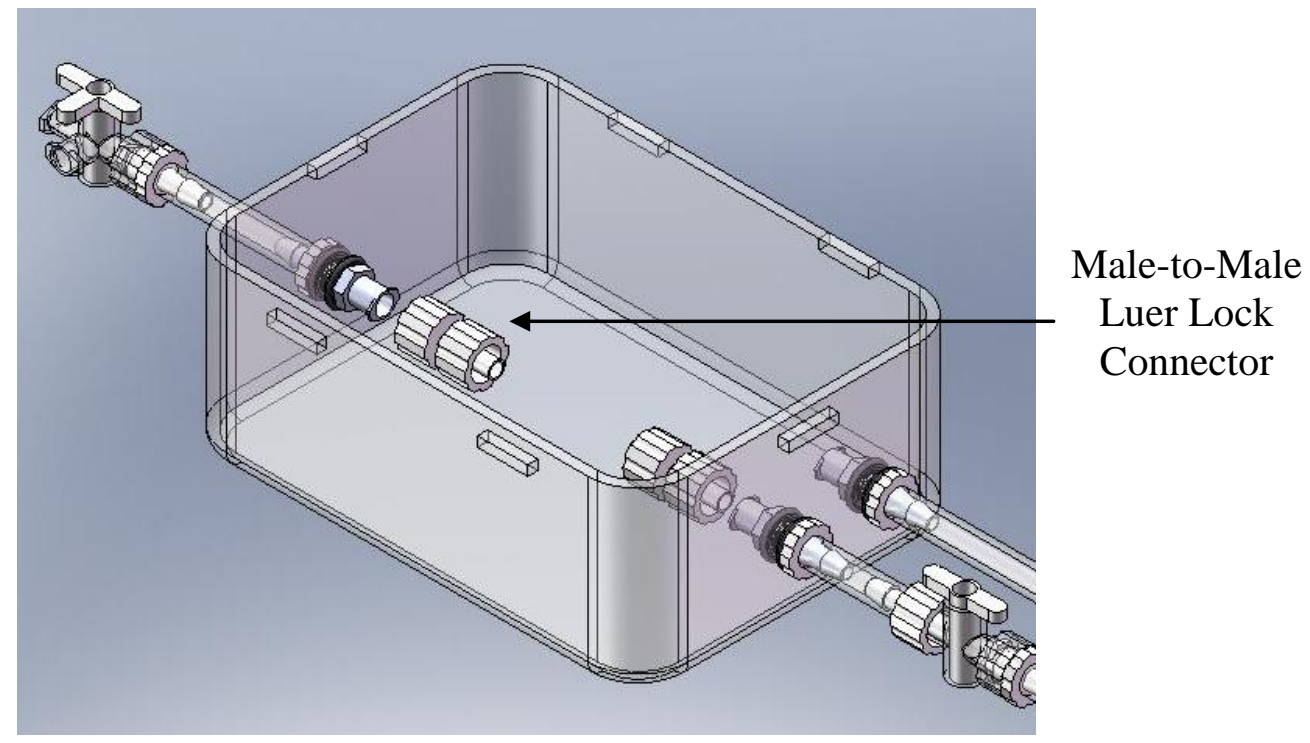

Figure 3.8- Attachment of male-to-male luer lock connectors. Male-to-male luer lock connectors, with one rotating collar, were attached to the female luer lock connector end of the luminal inlet and luminal outlet.

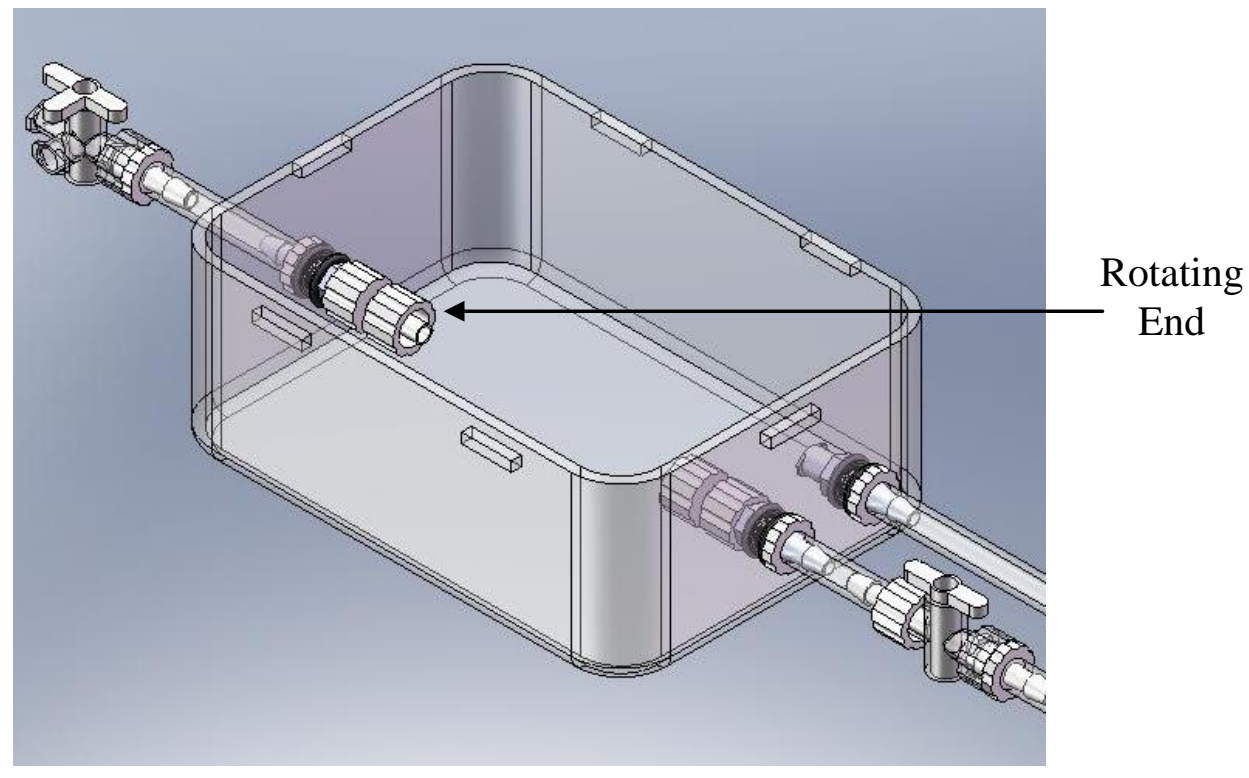

Figure 3.9- Completed attachment of male-to-male luer lock connectors. Male-to-male luer lock connectors were attached so that the end extending into the chamber remained free to rotate. 


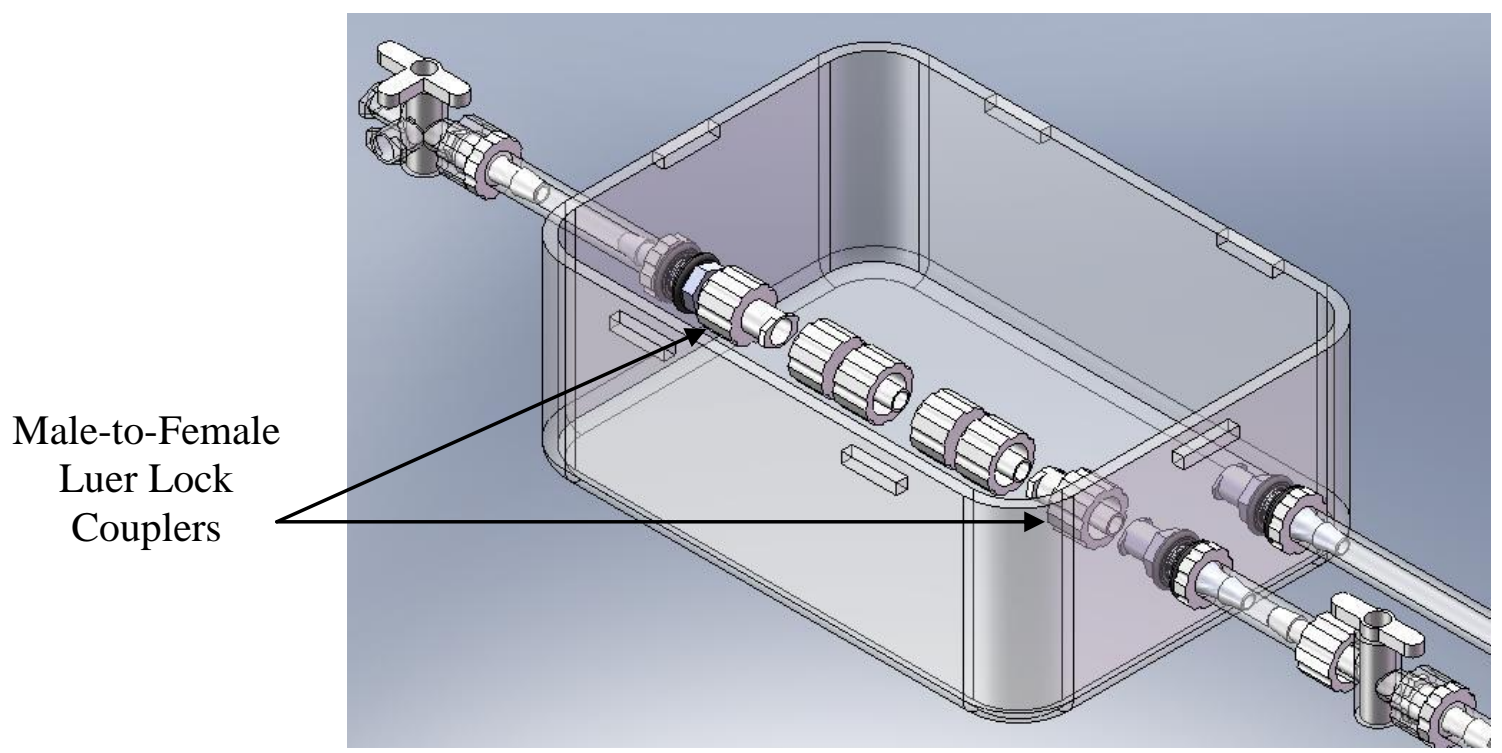

Figure 3.10- Addition of male-to-female luer lock couplers. Prior to the attachment of the male-to-male luer lock connectors, male-to-female couplers can be added to shorten the distance between the rotating ends.

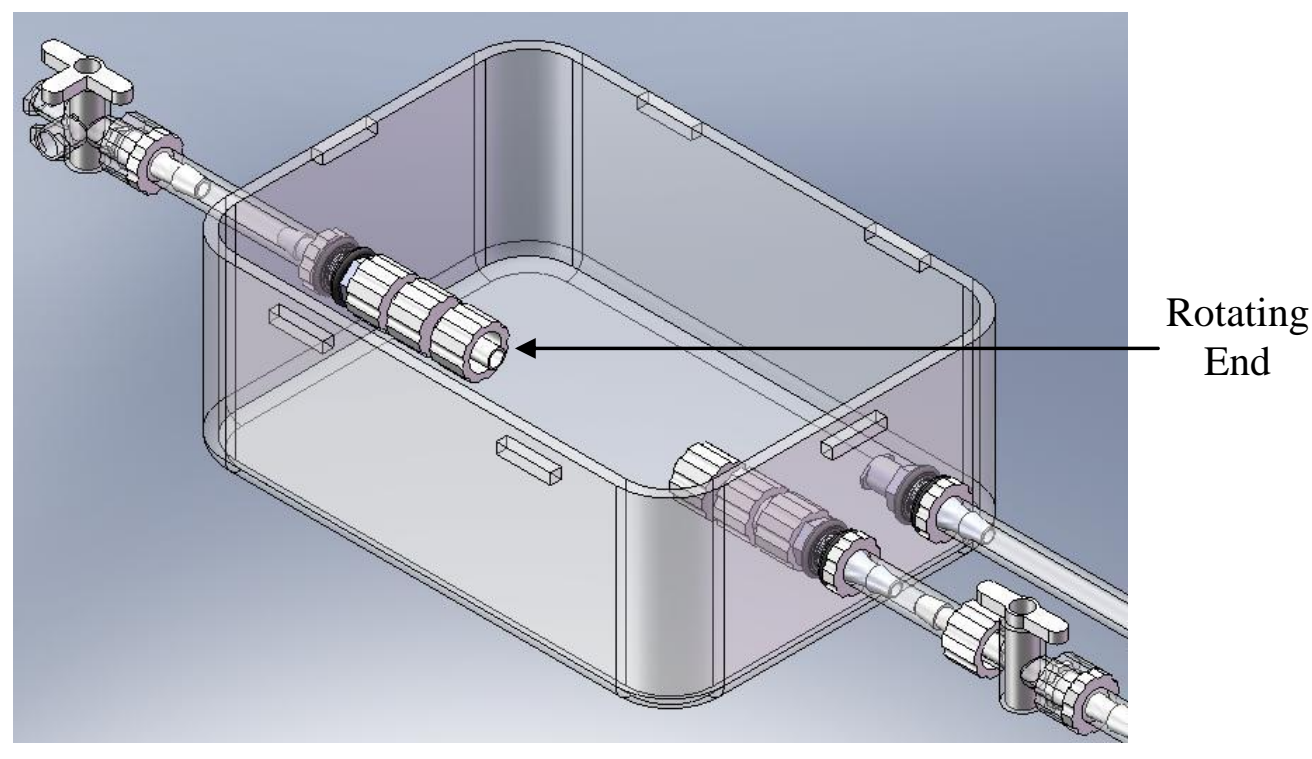

Figure 3.11- Attachment of male-to-female luer lock couplers. Male-to-male luer lock connectors were attached so that the end extending into the chamber remained free to rotate. 
The final complete new Ideal BVM system, prior to scaffold insertion, is diagramed below in Figure 3.12. The lid is attached by closing four snapping latches and a built-in o-ring creates a leak-proof seal. The Ideal BVM system was part of the overall bioreactor configuration, which will be diagramed in a later section.

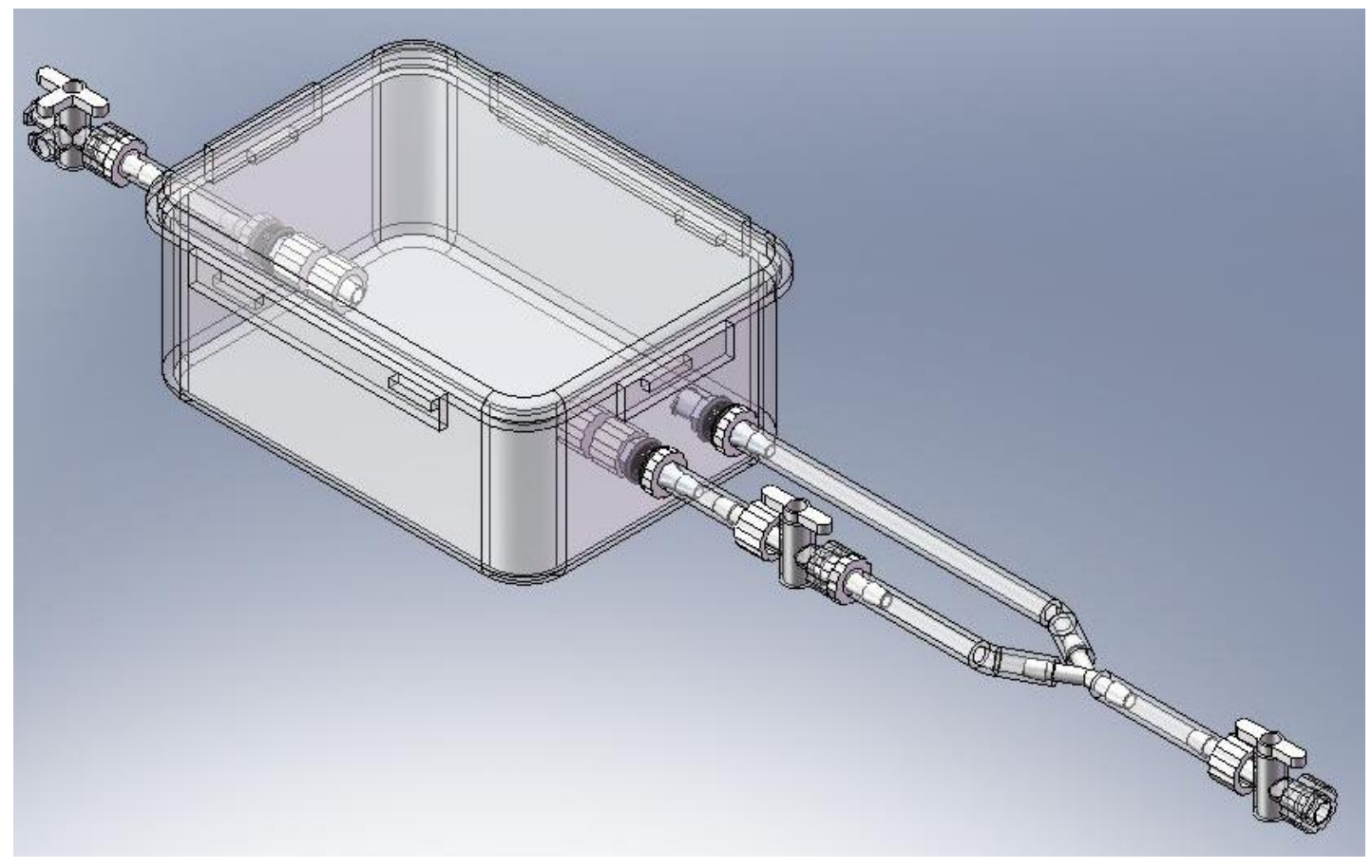

Figure 3.12- Final complete new Ideal BVM system. 
Two-Port Media Reservoir

This section outlines the steps taken to build the two-port media reservoir for the new Ideal BVM system. After construction of the luminal inlet and luminal outlets of the Ideal BVM system it was observed that this configuration would also improve the function and use of the inlet and outlet ports on the media reservoir lid, allowing reservoir construction to be accomplished without the need for a large amount of adhesive.

Therefore, the same basic construction used to build the Ideal BVM system luminal inlet and luminal outlets was applied to the two-port media reservoir. The assembly process is described in detail and includes the specific parts used along with detailed illustrations. While this section provides the materials and methods for construction of the two-port media reservoir, a detailed list of parts and drawings, as well as complete step-by-step directions can also be found in Appendix B and D respectively.

The two-port media reservoir for the Ideal BVM system was created as follows. Two 15/64" holes were drilled into the lid of a BD Falcon ${ }^{\mathrm{TM}} 50 \mathrm{ml}$ conical-bottom polypropylene tube (BD Biosciences P/N 352098). Using a razor blade, the edges of the holes were cleaned of any plastic debris that resulted from the use of the drill. Using the same technique described previously, a soft black Buna-N o-ring, AS568A dash number 006, durometer 50A, 1/4" OD, 1/8" ID, 1/16" W (McMaster-Carr P/N 2418T112) was slid onto a white nylon, female luer lug style panel mount connector, 1/4-28 UNF to 200 series barb, 1/8" ID tubing (Value Plastics P/N FTLB230-1) and threaded through one of the drilled holes with the barbed portion exiting the top of the lid. A second o-ring was placed over the barbed section and a white nylon, 1/4-28 UNF panel mount lock nut 
(Value Plastics P/N LNS-1) was screwed on tightly, pressing the o-rings against the top and bottom of the lid, creating a leak-proof seal. Once securely tightened, a drop of Loctite 4011 medical grade adhesive (McMaster-Carr P/N 1818A4) was placed between the lock nut and the threaded portion of the barbed fitting to secure it in place and prevent loosening. This procedure was repeated for the second drilled hole. Next, a 1" piece of Tygon ${ }^{\circledR}$ silicone tubing, 1/8" ID x 1/4" OD (Cole-Parmer P/N WU-95702-06) was attached to a white nylon, male luer integral lock ring to 200 series barb, 1/8" ID tubing (Value Plastics P/N MTLL230-1) and secured to one of the female luer fittings extending from the bottom of the lid. This port, with the attached tubing, became the outlet. The assembly process described is illustrated in Figures 3.13 and 3.14 below.

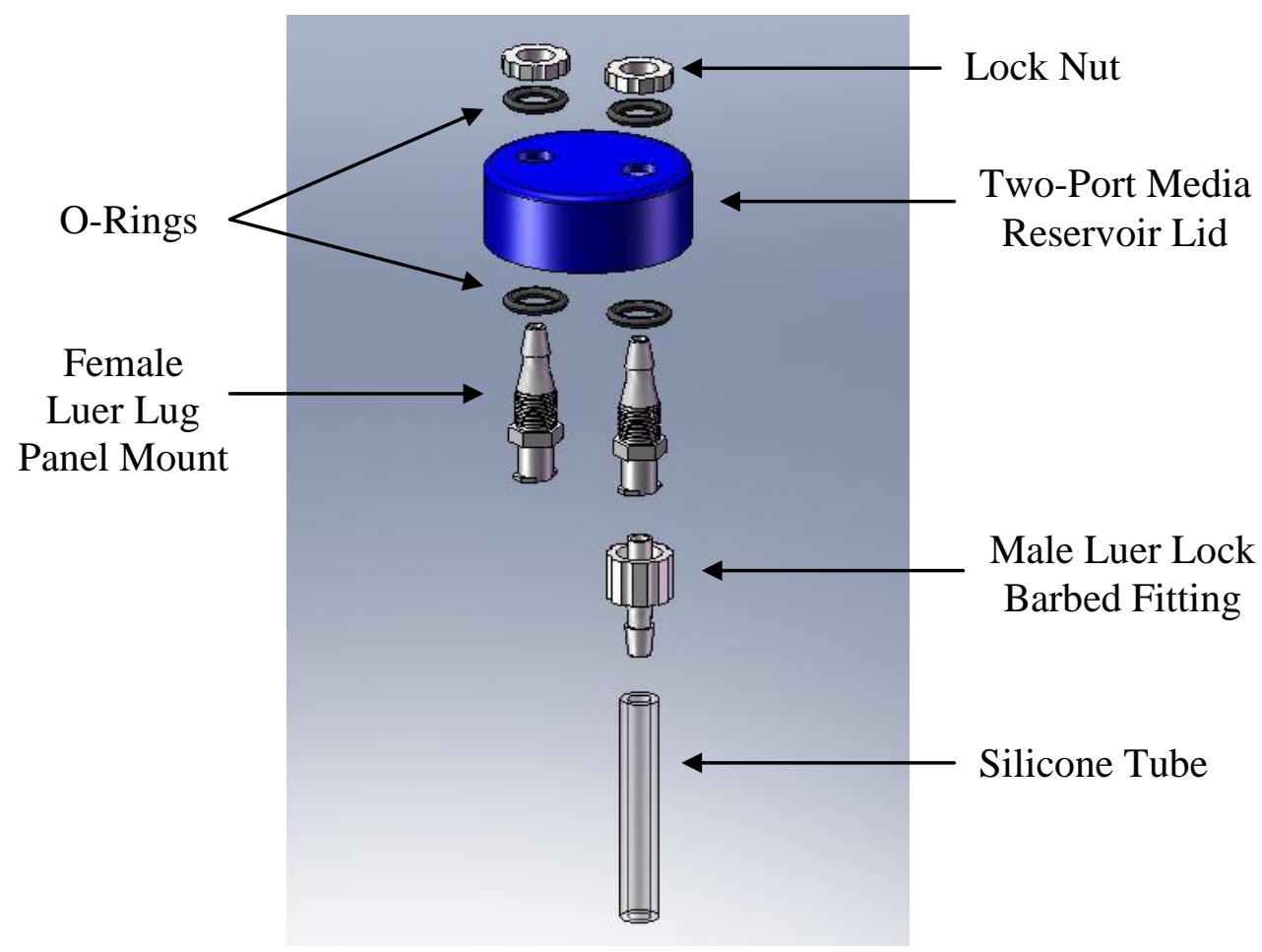

Figure 3.13- Assembly of two-port media reservoir lid. Each of the two 15/64" holes was assembled using two o-rings, a female luer lug panel mount and a lock nut. A short piece of tubing was inserted onto a male luer lock barbed fitting and attached to the assembly. 


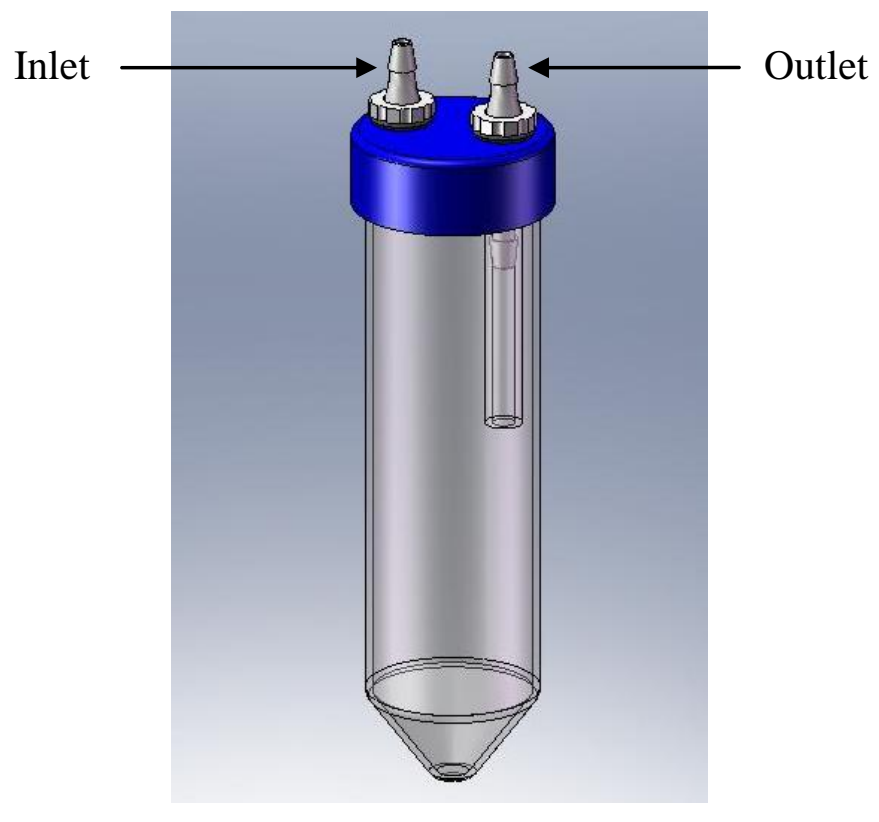

Figure 3.14- Assembled two-port media reservoir. The female luer lug panel mounts were threaded through the drilled holes and the lock nuts were tightened, pressing the o-rings against the top and bottom of the lid, creating a seal. A short piece of silicone tubing was attached and became the outlet.

To complete the two-port media reservoir two 10" pieces of Tygon® silicone tubing, 1/8" ID x 1/4" OD (Cole-Parmer P/N WU-95702-06) were inserted onto the barbed fittings of the inlet and outlet exiting the media reservoir lid. A white nylon, male luer integral lock ring to 200 series barb, 1/8" ID tubing (Value Plastics P/N MTLL2301) was attached to the open end of the silicone tubing attached to the outlet. Likewise, a white nylon, female luer thread style to 200 series barb, 1/8" ID tubing (Value Plastics P/N FTLL230-1) was attached to the open end of the silicone tubing attached to the inlet. Figures 3.15 and 3.16 below illustrate this assembly procedure. The two-port media reservoir was part of the overall bioreactor configuration, which will be diagramed in a later section. 


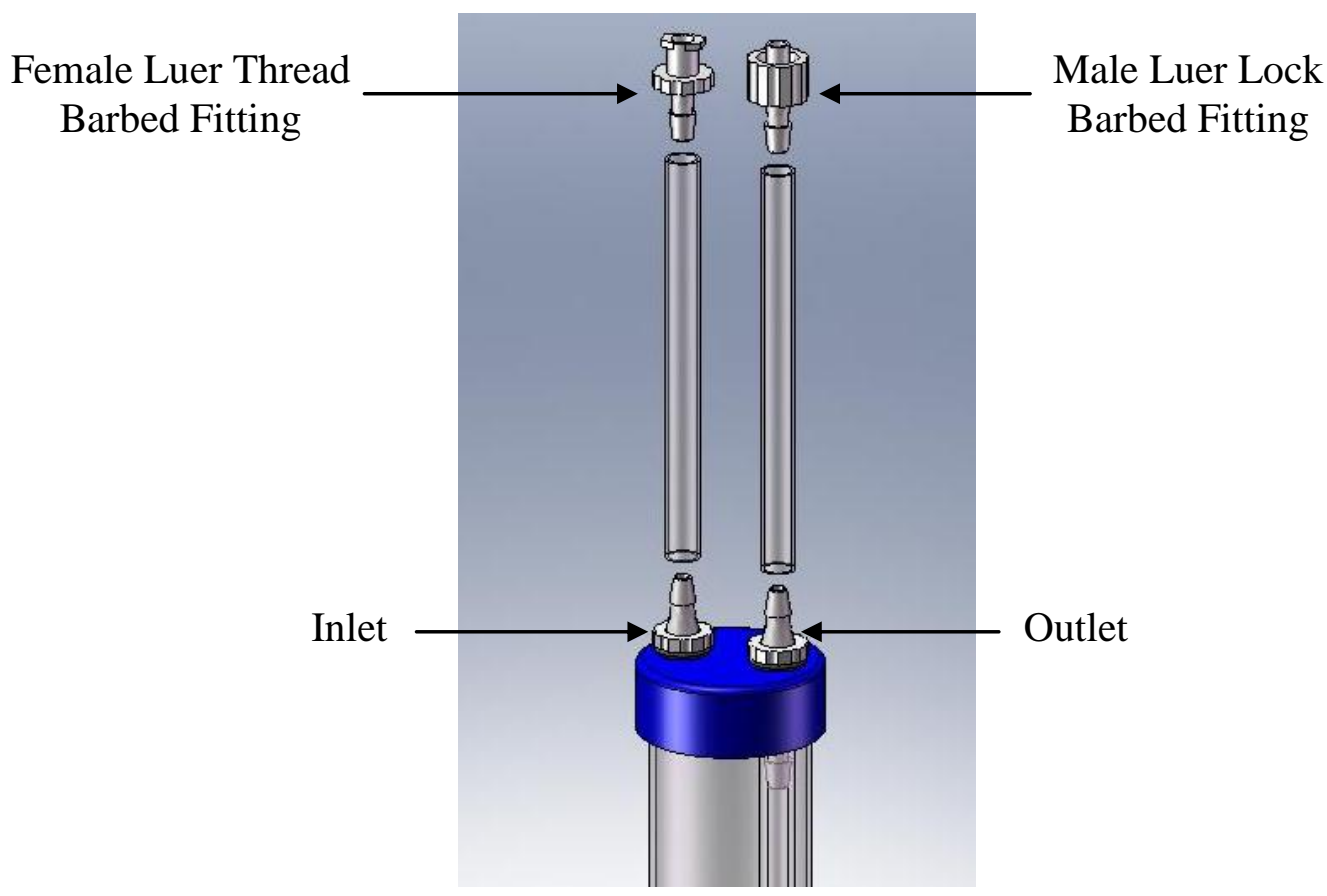

Figure 3.15- Inlet and outlet tubing assembly of two-port media reservoir. A male luer lock barbed fitting is attached to the outlet and a female luer thread barbed fitting is attached to the inlet.

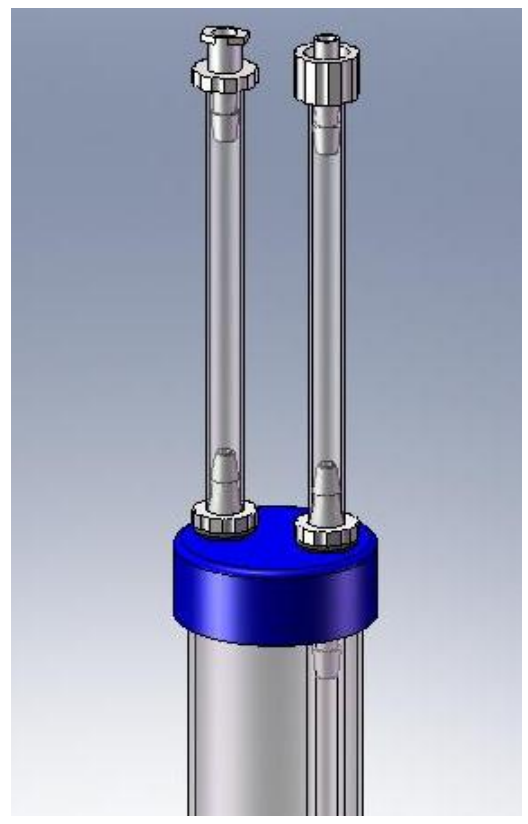

Figure 3.16- Final completed assembly of two-port media reservoir. 
Scaffold Setup and Insertion

The scaffold for the new Ideal BVM system was setup as follows. Expanded polytetrafluoroethylene (ePTFE), $3 \mathrm{~mm}$ and $4 \mathrm{~mm}$ inner diameter (Impra Bard, Inc., Tempe, AZ), was used as the scaffold material. Two white nylon, female luer thread style to 200 series barb fittings were inserted into the open ends of the ePTFE scaffold. White nylon, female luer thread style to 200 series barb, 1/8" ID tubing (Value Plastics P/N FTLL230-1) were used for the $4 \mathrm{~mm}$ inner diameter scaffolds and white nylon, female luer thread style to 200 series barb, 3/32" ID tubing (Value Plastics P/N FTLL220-1) were used for the $3 \mathrm{~mm}$ inner diameter scaffolds. The ends of the scaffold were sutured onto the barbed ends of the female luer thread fittings using 2.0 Sofsilk ${ }^{\mathrm{TM}}$ suture material. Figures 3.17 and 3.18 below illustrate this procedure. Depending on the number of male luer integral lock ring to female luer thread style couplers used between male-to-male rotating fittings, scaffolds were cut into either $5.5 \mathrm{~cm}, 4.5 \mathrm{~cm}$ or $3.5 \mathrm{~cm}$ lengths, corresponding to 0,1 , or 2 couplers respectively. See Table 3.1 below or Appendix E for a summary of scaffold length versus number of couplers used.

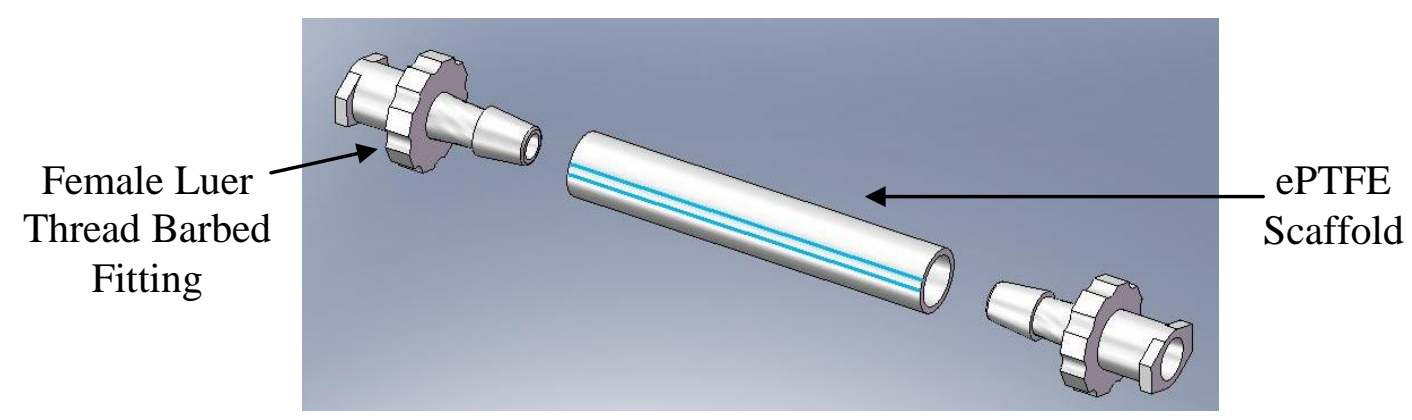

Figure 3.17- Scaffold assembly. Two female luer thread barbed fittings were inserted into the open ends of the ePTFE scaffold. 


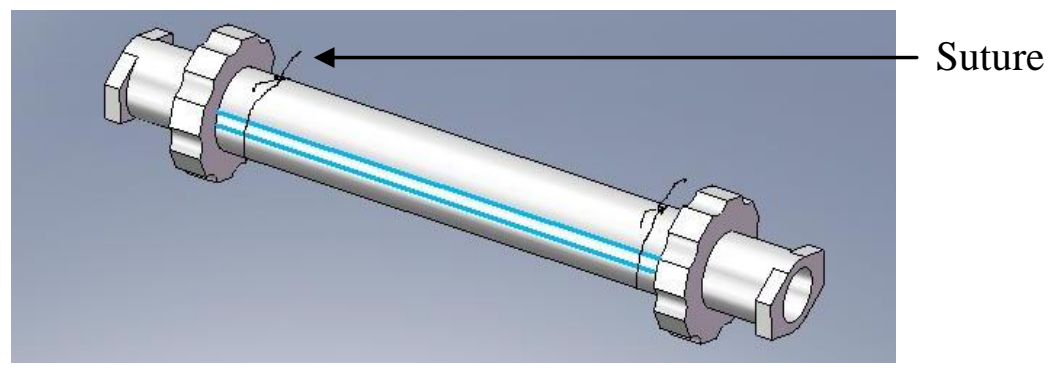

Figure 3.18- Completed scaffold assembly. The ends of the ePTFE scaffold were sutured to the two female luer thread barbed fittings.

\begin{tabular}{|c|c|c|}
\hline Illustration & \# of Couplers & Scaffold Length \\
\hline & 0 & $5.5 \mathrm{~cm}$ \\
\hline & & \\
\hline & & \\
\hline
\end{tabular}

Table 3.1- Scaffold length versus number of couplers used. 
To secure the scaffold inside the Ideal BVM system, the female luer thread fittings attached to the scaffold were inserted into the male-to-male luer lock fittings attached to the luminal inlet and luminal outlet. The rotating collars of the male-to-male luer lock fittings were tightened around the female luer thread fittings attached to the scaffold. The inserted scaffold is illustrated below in Figure 3.19.

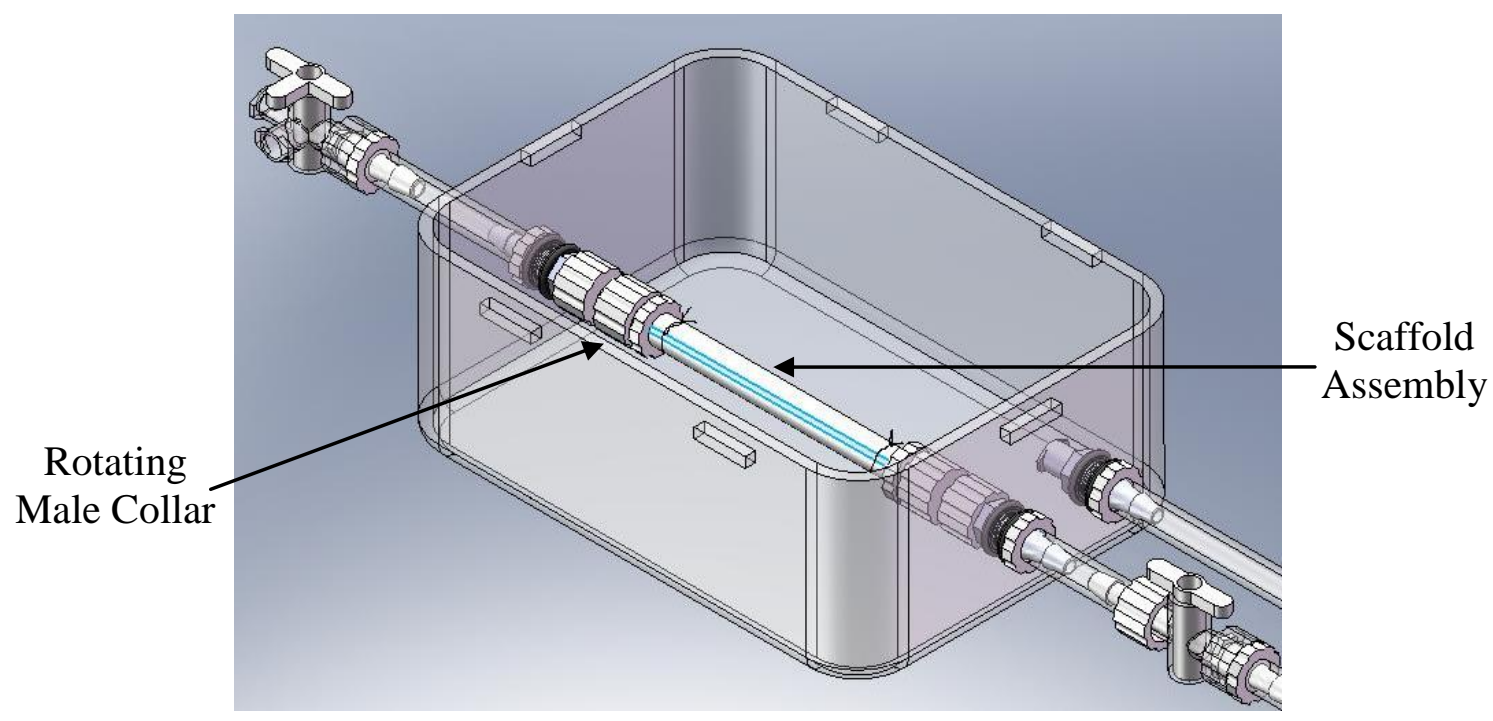

Figure 3.19- Scaffold insertion. The scaffold is secured inside the BVM system by tightening the rotating collars of the male-to-male luer lock fittings around the female luer thread fittings attached to the scaffold.

Bioreactor Configuration

The complete bioreactor configuration consisted of the Ideal BVM system and two-port media reservoir connected in series. To construct the bioreactor system the male luer lock fitting attached to the two-port media reservoir outlet tubing was secured to the female luer thread fitting of the three-way stopcock attached to the luminal inlet of the Ideal BVM system. Next, the female luer thread fitting attached to the two-port media 
reservoir inlet tubing was secured to the male luer lock fitting of the one-way stopcock attached to the main luminal outlet of the Ideal BVM system. This completed the Ideal BVM system bioreactor assembly. The Ideal BVM bioreactor system is illustrated in Figure 3.20 below.

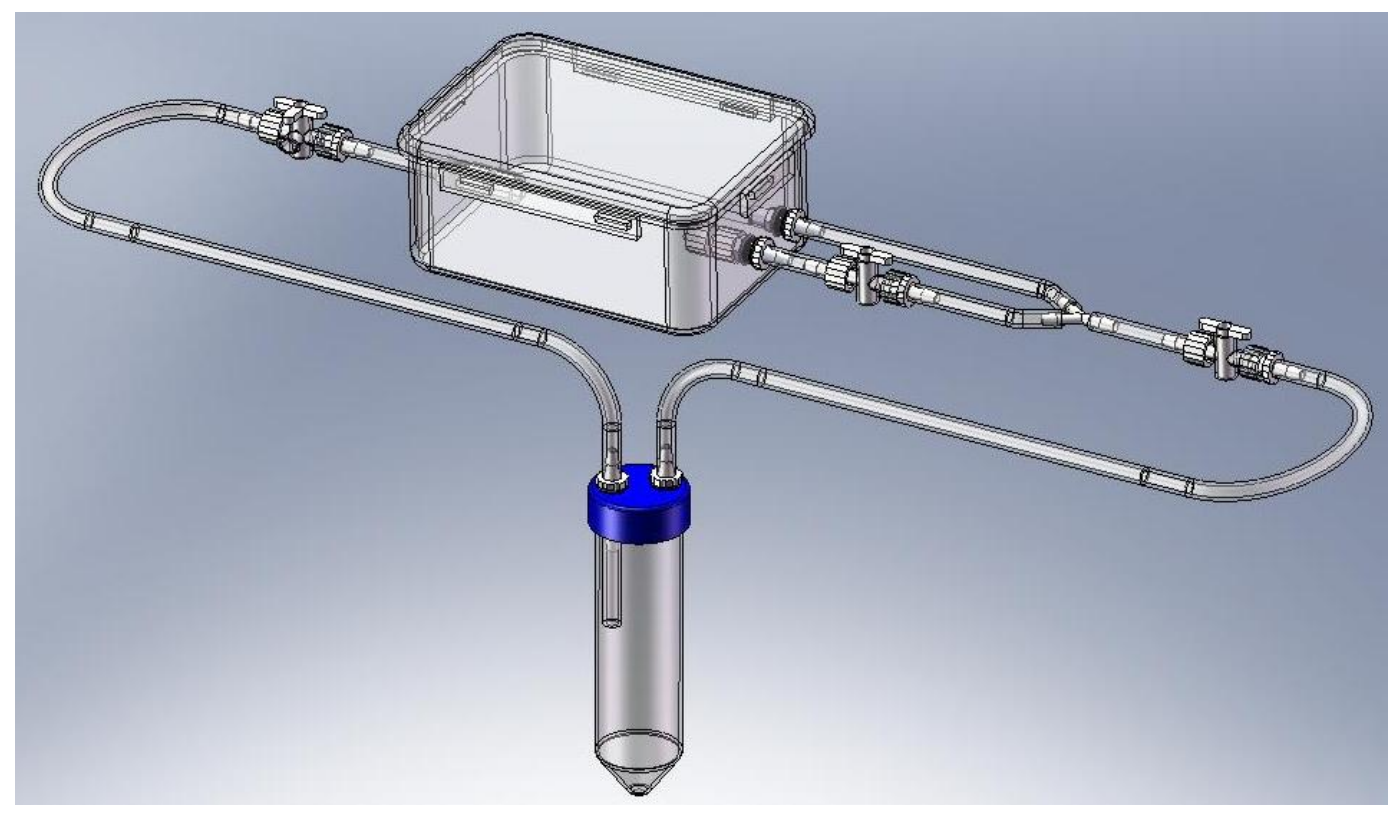

Figure 3.20- Ideal BVM system bioreactor configuration.

\section{Cost Analysis}

Due to the goal of creating a cost-effective, high-throughput testing environment, the cost of each individual component and part used to create the Ideal BVM system and two-port media reservoir was determined. The cost of all components and parts were summed and the total cost per individual completed Ideal BVM system and two-port media reservoir was calculated. 


\section{Functional Verification}

To verify that the Ideal BVM system and two-port media reservoir functioned properly without leakage, a complete bioreactor configuration was setup and used. A lab member completed a full sterile Ideal BVM setup including scaffold assembly and insertion, media injection, and connection of the Ideal BVM and two-port media reservoir in series. Flow through the bioreactor system was initiated by placing a section of the two-port media outlet tubing through a peristaltic pump. The Ideal BVM functional verification setup is illustrated in Figure 3.21 below.

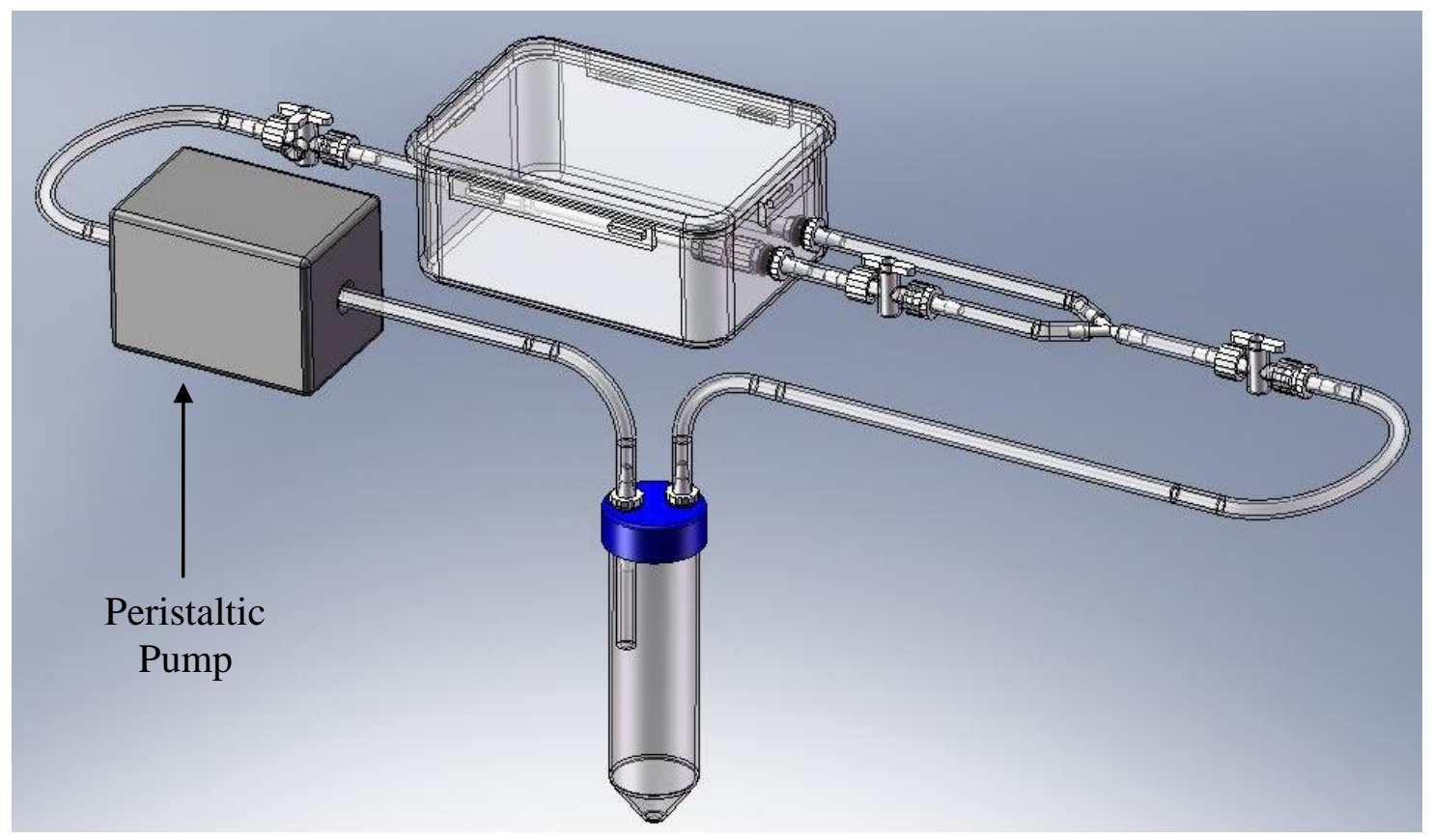

Figure 3.21- Ideal BVM functional verification setup. Flow was initiated by placing a section of the two-port media outlet tubing through a peristaltic pump. 


\section{Comparison of Ideal BVM System to Original BVM System}

To compare the Ideal BVM system and the original BVM system a lab member completed a full sterile side-by-side setup of both systems. Setup included scaffold assembly and insertion, media injection, and connection of the BVM systems and twoport media reservoirs in series. Flow through the bioreactor systems was initiated by placing a section of the two-port media outlet tubing through a peristaltic pump. The Ideal BVM system and original BVM system were compared and user feedback was gathered on overall usability, ease of scaffold insertion, user interface features, connections and fittings, bioreactor assembly, seal-ability, aseptic use and setup, and general component configuration. The user feedback survey can be found in Appendix F.

\section{Blood Vessel Development}

The Ideal BVM system was tested for its ability to cultivate an in vitro blood vessel mimic. Following scaffold insertion and priming of the system with media, human umbilical artery smooth muscle cells (HUASMC) were pressure sodded onto the luminal surface of the ePTFE scaffold. The BVM system was placed in an incubator at $37{ }^{\circ} \mathrm{C}$, $5 \% \mathrm{CO}_{2}$, and connected to a peristaltic pump. The pump generated flow through the lumen of the vessel over a time period of one day. After one day, the vessel was removed from the chamber and fixed in Histochoice for evaluation. Cell deposition and cell adherence to the vessel were evaluated using a scanning electron microscope. The fixed vessel was cut in half longitudinally and images of the luminal surface were acquired to 
determine if the Ideal BVM system was capable of successfully supporting cell sodding and cultivation. 
Results

Ideal BVM System

The Ideal BVM System was easy to assemble and build. All components were off the shelf and readily available, resulting in a design that was repeatable and consistent. No special tooling was needed and assembly was simple and straightforward. The leakproof snap and seal container was easy to work with and durable. Holes were drilled cleanly with a handheld power drill and an ordinary 15/64" drill bit. By threading the female luer lug panel mounts directly into the plastic the need for a large amount of adhesive was eliminated. The double o-ring design created a leak-proof seal and allowed for a clean and simple overall design. Tubing was easily attached to the barbed fittings exiting the chamber and female luer threads allowed for easy attachment of male-to-male rotating luer lock fittings. Male luer lock and female luer thread connectors and barbed fittings made tubing and stopcock assembly easy. Due to the simplicity of design, availability of components and easy assembly, it was possible to construct a large number of identical systems. Therefore, thirty Ideal BVM systems were constructed for immediate use. Assembled Ideal BVM systems are shown below in Figures 3.22 and 3.23 . 


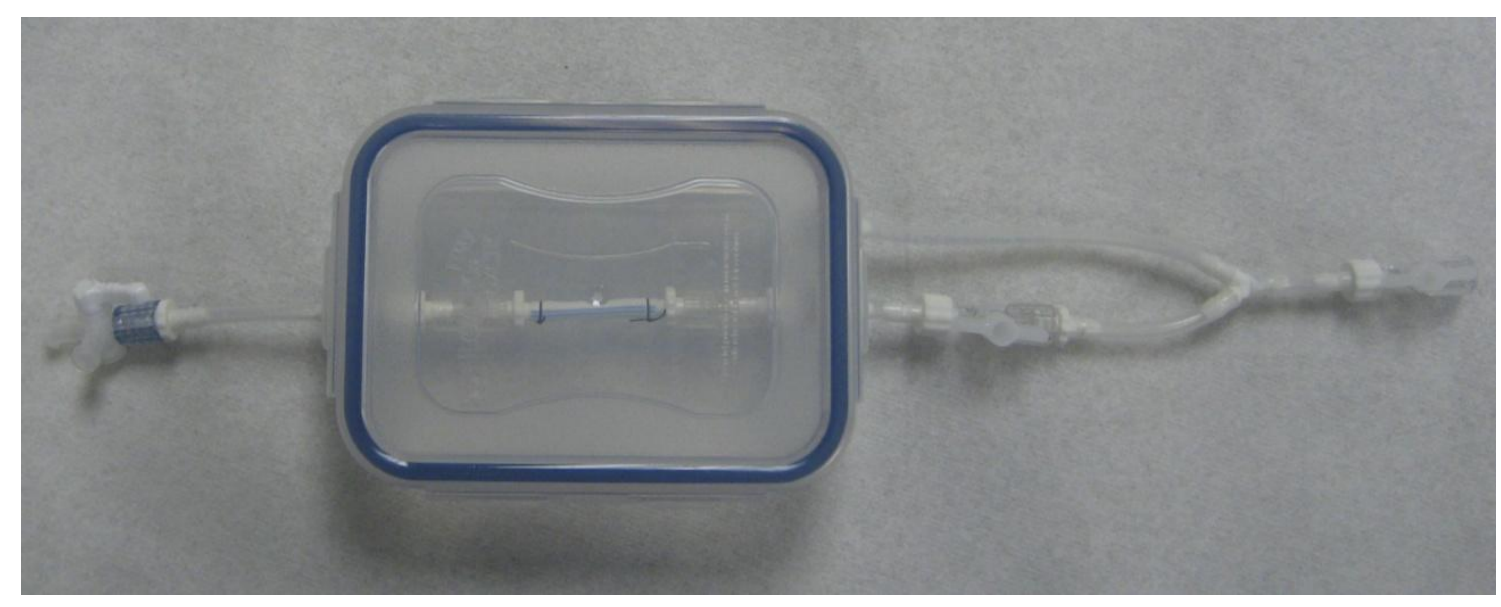

Figure 3.22- Ideal BVM System.

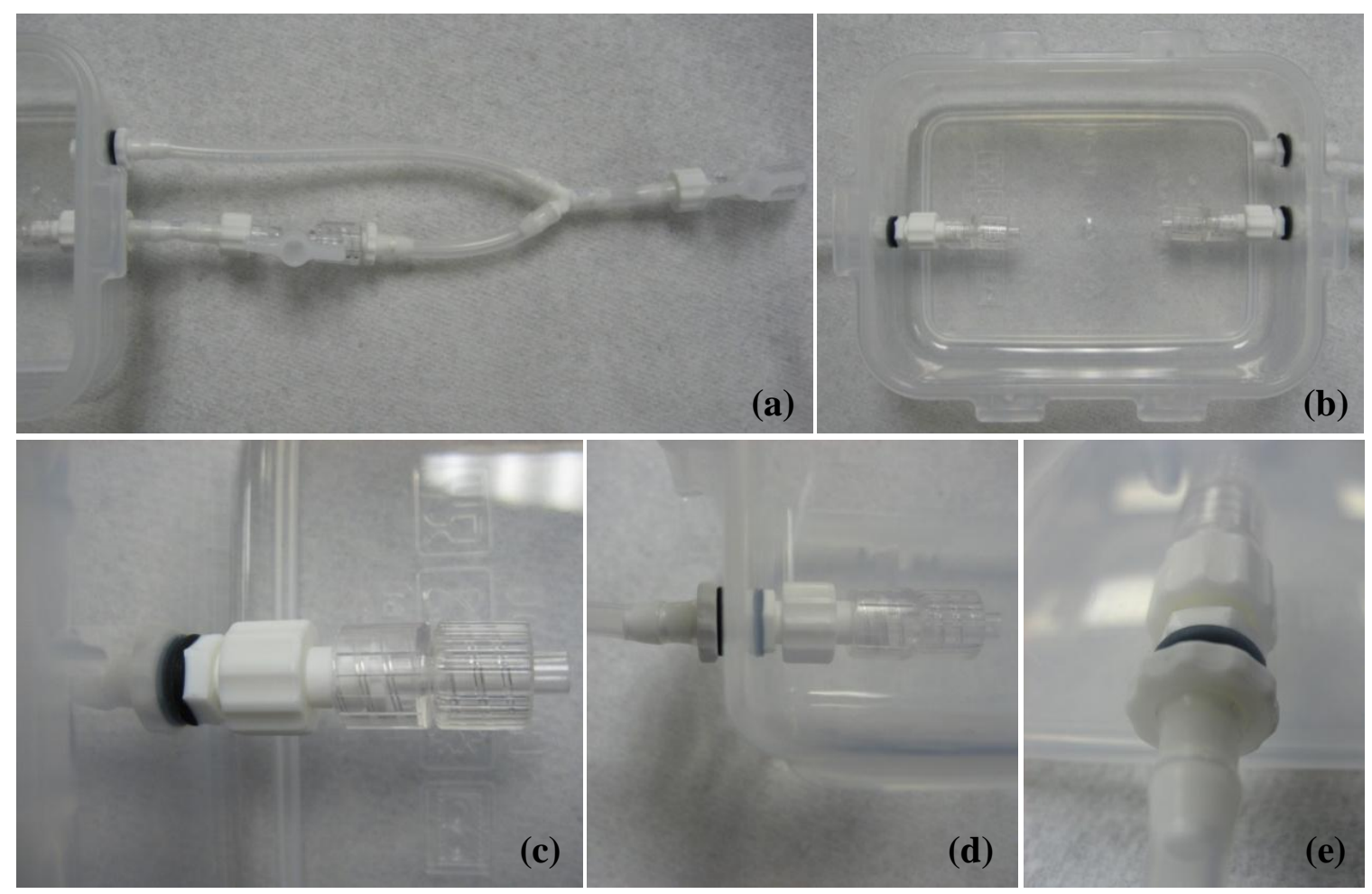

Figure 3.23- Assembled Ideal BVM systems. Tubing was attached to the barbed fittings exiting the sides of the container (a) and male-to-male rotating fittings were attached to the female luer thread fittings extending into the chamber (b) and (c). The sealing double o-ring design (d) eliminated the need for a large amount of adhesive (e). 
Two-Port Media Reservoir

Two-port media reservoir lids were also easy to assemble and build. The sealing double o-ring design eliminated the need for a large amount of adhesive and allowed for a clean and simple overall design, while providing a functional seal. Tubing was easily attached to the barbed fittings exiting the top of the lid and the $50 \mathrm{ml}$ tube was easy to screw onto the lid. The female luer thread fittings exiting the bottom of the lid also made it easy to attach a short piece of tubing to create the outlet. Due to the simplicity of design, availability of components and easy assembly, thirty two-port media reservoirs were constructed for immediate use. Assembled two-port media reservoirs are shown below in Figures 3.24 and 3.25.

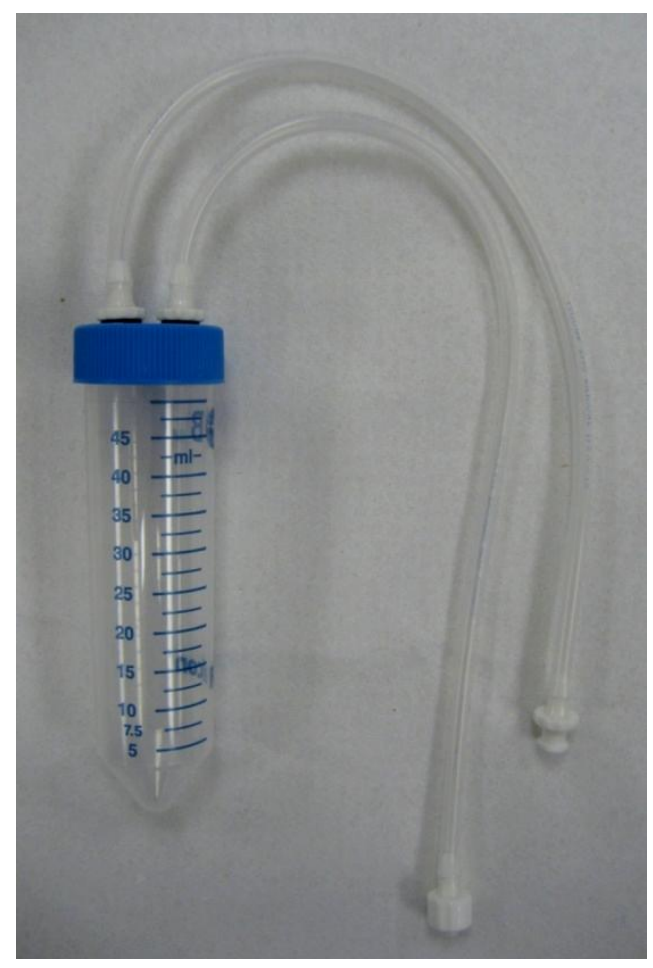

Figure 3.24- Two-port media reservoir. 

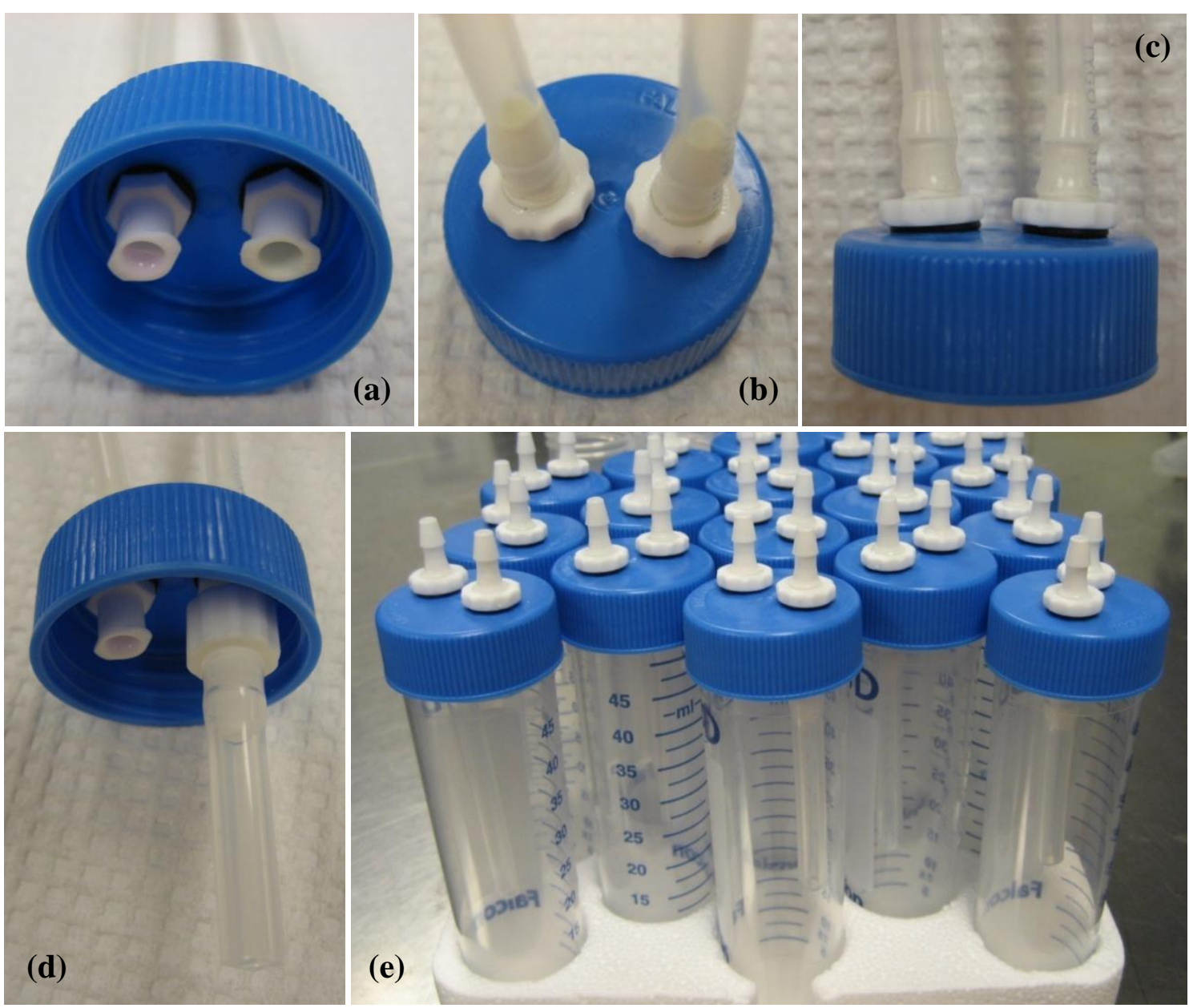

Figure 3.25- Assembled two-port media reservoirs. The sealing double o-ring design (a) eliminated the need for a large amount of adhesive (b). Tubing was attached to the barbed fittings exiting the top of the lid (c) and a short piece of tubing was secured to the female luer thread fitting exiting the bottom (d). Thirty two-port media reservoirs were constructed for immediate use (e).

Scaffold Setup and Insertion

Scaffold setup was simple and straightforward. The female luer thread barbed fittings were easy to insert into the ePTFE scaffold and the Sofsilk ${ }^{\mathrm{TM}}$ suture material was easy to tie and successfully secured the scaffold in place. The female luer thread barbed fittings were also easy to insert into the male-to-male rotating fittings within the Ideal 
BVM chamber. The rotating collars of the male-to-male luer lock fittings allowed insertion to be performed without bending or twisting of the scaffold material. The female luer thread end attached to the scaffold could be held in place while the male luer lock rotating collar was tightened around it. These features also permitted fast and asceptic insertion of the scaffold within the Ideal BVM chamber. Scaffold setup and insertion are shown below in Figures 3.26 and 3.27.

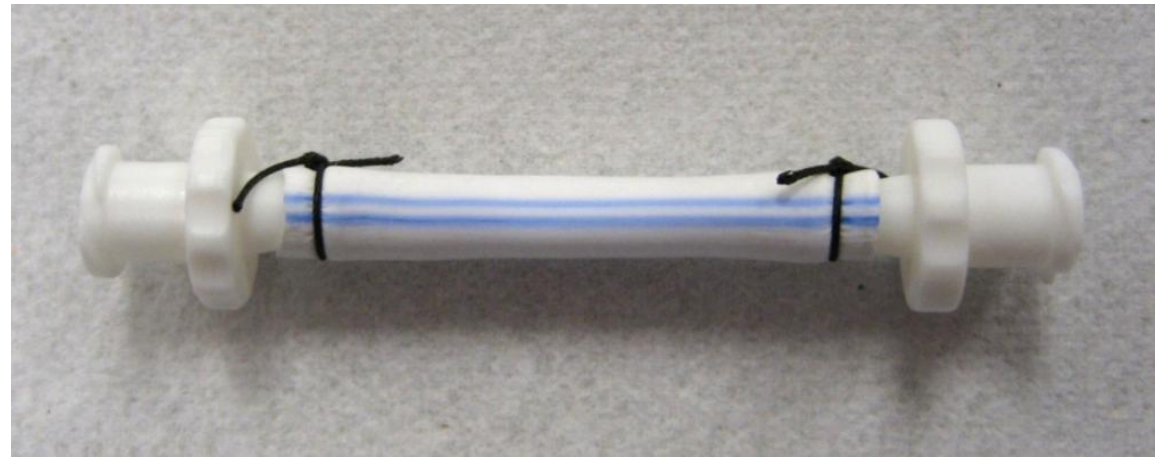

Figure 3.26- Scaffold assembly.

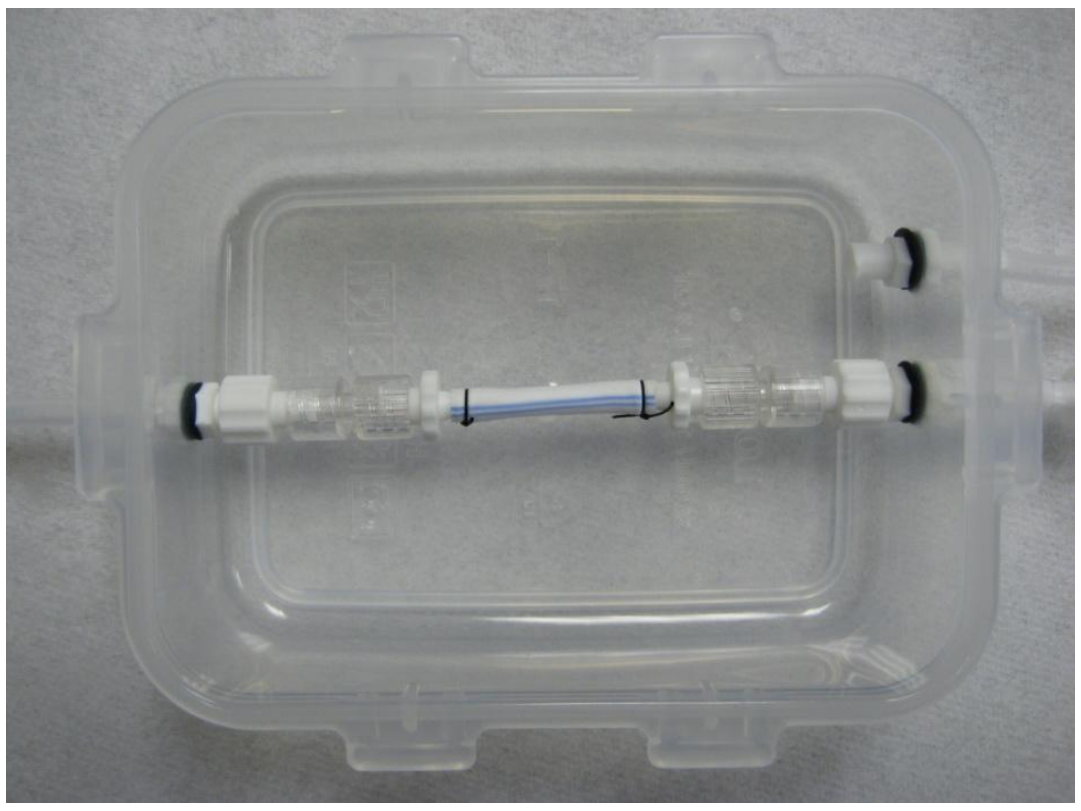

Figure 3.27- Scaffold insertion into Ideal BVM system. 
An advantage of this new scaffold setup was that scaffold length and diameter were easily adjustable and variations were easily accommodated within the system. By choosing the correct size of female luer thread barbed fittings a number of diameters of ePTFE scaffold could be used. In addition, by using Table 3.1, scaffold length was easily adjustable by varying the number of couplers used in the inner BVM chamber assembly. The ability to vary scaffold length and diameter allows for the accommodation a large number of stents sizes to be tested within the Ideal BVM system.

\section{Bioreactor Configuration}

The complete bioreactor configuration, consisting of the Ideal BVM system and two-port media reservoir connected in series, was easy to setup with the need for only two simple connections. Specific placement of male luer lock and female luer thread fittings ensured proper setup and orientation every time. Only one configuration existed for setting up the bioreactor system, since the fittings of the Ideal BVM system and twoport media reservoir were designed as male-to-female connections. This new design eliminated the confusion and complications faced in determining the correct inlet and outlet orientation when connecting the two-port media reservoir to the Ideal BVM system. The complete Ideal BVM bioreactor configuration is shown below in Figure 3.28 . 


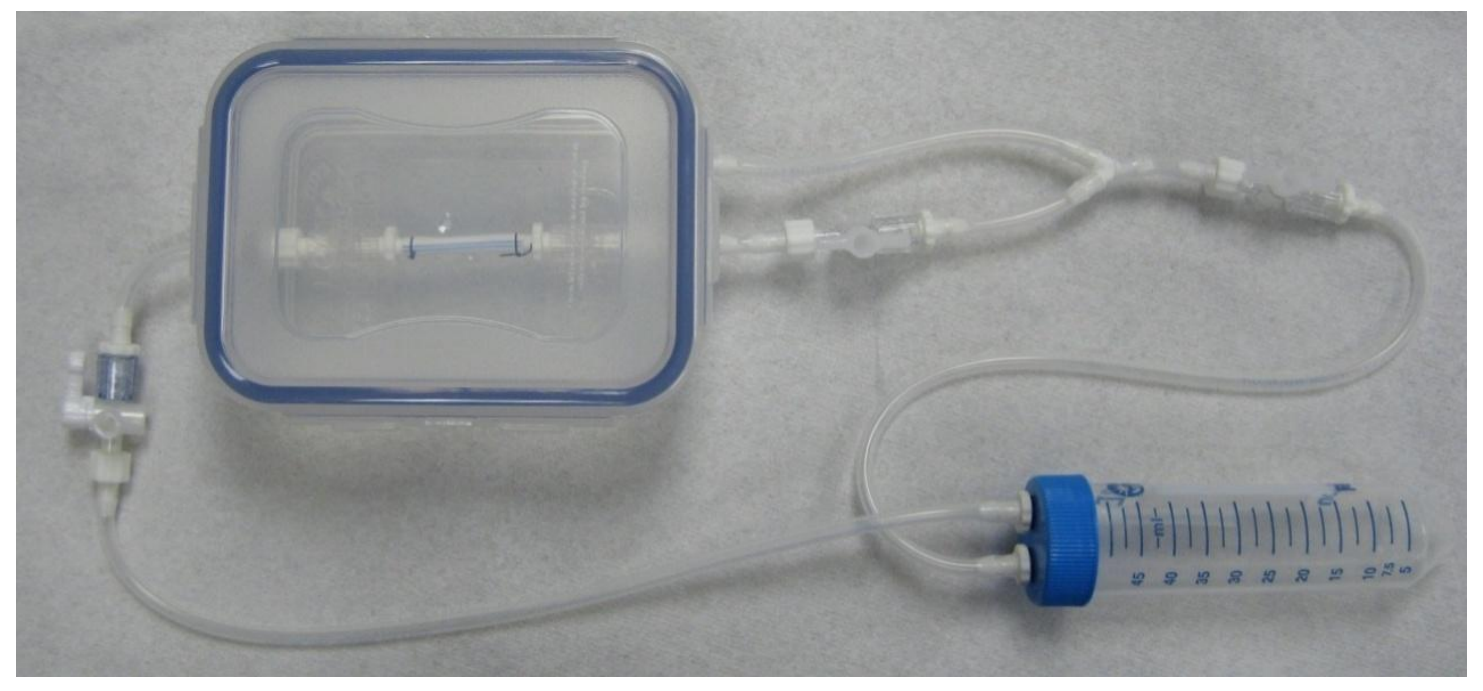

Figure 3.28- Complete bioreactor configuration. The Ideal BVM system and two-port media reservoir were connected in series. Specific placement of male luer lock and female luer thread fittings ensured proper setup and orientation every time.

Cost Analysis

The cost of each individual component and part used to create the Ideal BVM system and two-port media reservoir and the total cost per individual completed Ideal BVM system and two-port media reservoir is summarized in Tables 3.2 and 3.3 below. The total cost per individual completed Ideal BVM system was $\$ 9.71$ and the total cost per individual completed two-port media reservoir was $\$ 5.54$. The total cost of the completed bioreactor configuration, including both the Ideal BVM system and two-port media reservoir was $\$ 15.25$. 


\begin{tabular}{|c|c|c|c|c|c|}
\hline Component & Company & Part Number & $\begin{array}{c}\text { Qty per } \\
\text { BVM }\end{array}$ & $\begin{array}{c}\text { Cost per } \\
\text { Unit }\end{array}$ & Total \\
\hline Leak-Proof Chamber & Lock \& Lock & HPL806 & 1 EA & 3.00 & 3.00 \\
\hline Buna-N O-Ring & McMaster-Carr & 2418T112 & 6 EA & 0.07 & 0.42 \\
\hline Loctite 4011 Adhesive & McMaster-Carr & 1818A4 & 3 drops & 0.02 & 0.06 \\
\hline $\begin{array}{c}\text { Female Luer Lug Panel } \\
\text { Mount-1/8" Barb }\end{array}$ & Value Plastics & FTLB230-1 & 3 EA & 0.17 & 0.51 \\
\hline Lock Nut & Value Plastics & LNS-1 & 3 EA & 0.08 & 0.24 \\
\hline $\begin{array}{c}\text { Male Luer Lock-1/8" } \\
\text { Barb }\end{array}$ & Value Plastics & MTLL230-1 & 2 EA & 0.14 & 0.28 \\
\hline $\begin{array}{c}\text { Female Luer Thread- } \\
\text { 1/8" Barb }\end{array}$ & Value Plastics & FTLL230-1 & 2 EA & 0.12 & 0.24 \\
\hline Y-Connector-1/8" Barbs & Value Plastics & Y230-1 & 1 EA & 0.22 & 0.22 \\
\hline Male-Female Coupler & Value Plastics & LC34-1 & 2 EA & 0.16 & 0.32 \\
\hline 1-Way Stopcock & Value Plastics & VP455980 & 2 EA & 0.64 & 1.28 \\
\hline 3-Way Stopcock & Value Plastics & VPB1000079N & 1 EA & 0.64 & 0.64 \\
\hline $\begin{array}{c}\text { Male-to-Male Rotating } \\
\text { Luer Lock }\end{array}$ & Qosina & 17664 & 2 EA & 0.26 & 0.52 \\
\hline 1/8" ID Silicone Tubing & Cole-Parmer & WU-95702-06 & 11 inches & 0.18 & 1.98 \\
\hline \multicolumn{2}{|r|}{} & & & $\$ 9.71$ \\
\hline
\end{tabular}

Table 3.2- Total cost per individual completed Ideal BVM system.

\begin{tabular}{|c|c|c|c|c|c|}
\hline Component & Company & Part Number & $\begin{array}{l}\text { Qty per } \\
\text { Reservoir }\end{array}$ & $\begin{array}{l}\text { Cost per } \\
\text { Unit }\end{array}$ & Total \\
\hline $\begin{array}{l}\text { BD Falcon } \\
\text { Tube }\end{array}$ & BD Biosciences & 352098 & $1 \mathrm{EA}$ & 0.54 & 0.54 \\
\hline Buna-N O-Ring & McMaster-Carr & 2418T112 & $4 \mathrm{EA}$ & 0.07 & 0.28 \\
\hline Loctite 4011 Adhesive & McMaster-Carr & 1818A4 & 2 drops & 0.02 & 0.04 \\
\hline $\begin{array}{c}\text { Female Luer Lug Panel } \\
\text { Mount-1/8" Barb }\end{array}$ & Value Plastics & FTLB230-1 & $2 \mathrm{EA}$ & 0.17 & 0.34 \\
\hline Lock Nut & Value Plastics & LNS-1 & $2 \mathrm{EA}$ & 0.08 & 0.16 \\
\hline $\begin{array}{l}\text { Male Luer Lock-1/8" } \\
\text { Barb }\end{array}$ & Value Plastics & MTLL230-1 & $2 \mathrm{EA}$ & 0.14 & 0.28 \\
\hline $\begin{array}{c}\text { Female Luer Thread- } \\
\text { 1/8" Barb }\end{array}$ & Value Plastics & FTLL230-1 & $1 \mathrm{EA}$ & 0.12 & 0.12 \\
\hline 1/8" ID Silicone Tubing & Cole-Parmer & WU-95702-06 & 21 inches & 0.18 & 3.78 \\
\hline & & & & & $\$ 5.54$ \\
\hline
\end{tabular}

Table 3.3- Total cost per individual completed two-port media reservoir. 


\section{Functional Verification}

The Ideal BVM system was used in conjunction with a two-port media reservoir and functioned properly without any leakage. The scaffold was inserted aseptically and the lid was easy to attach and created a leak-proof seal. The system was injected with media which was pumped through the system via a peristaltic pump. Flow was created through the luminal inlet and exited the luminal outlet and extra luminal outlet. The Ideal BVM systems were EO sterilized prior to use. Photos of the Ideal BVM system being setup and being attached to the peristaltic pump are shown below in Figures 3.29 and 3.30 .
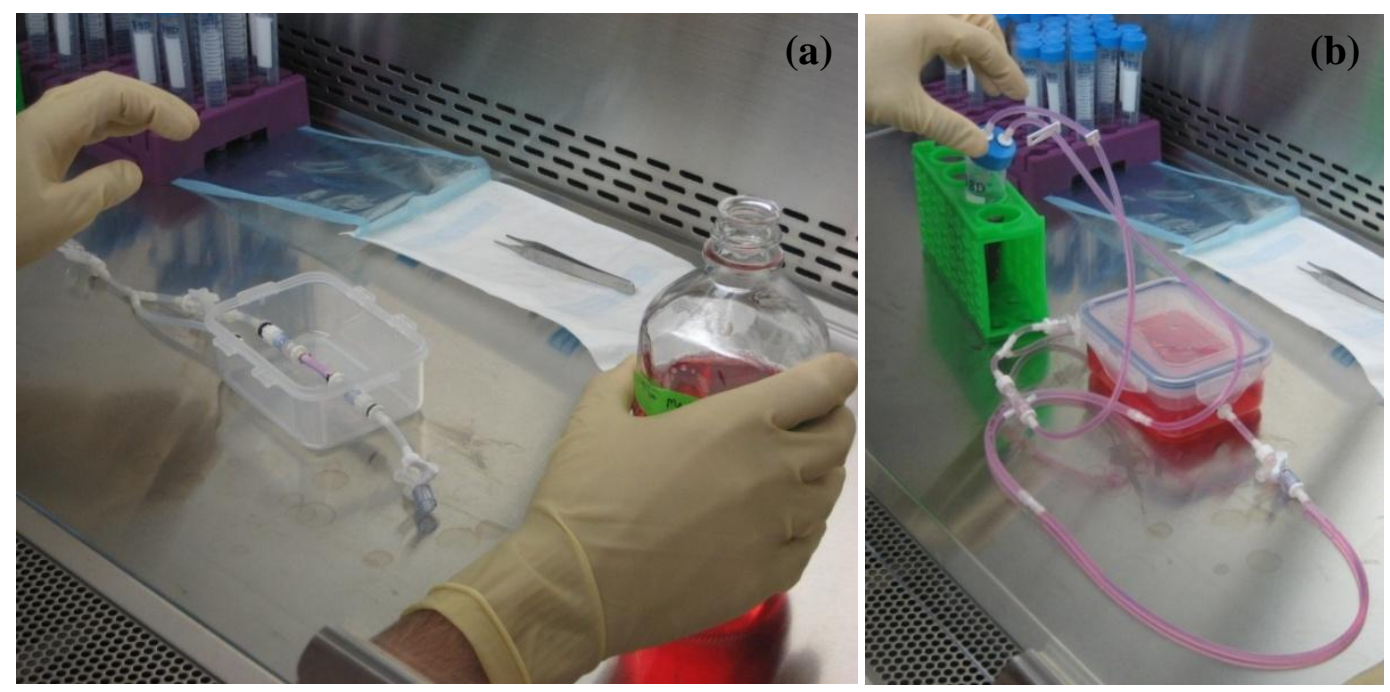

Figure 3.29- Setup of the Ideal BVM bioreactor system. The scaffold was inserted in an aseptic manner (a) and the two-port media reservoir and Ideal BVM were connected in series (b). 


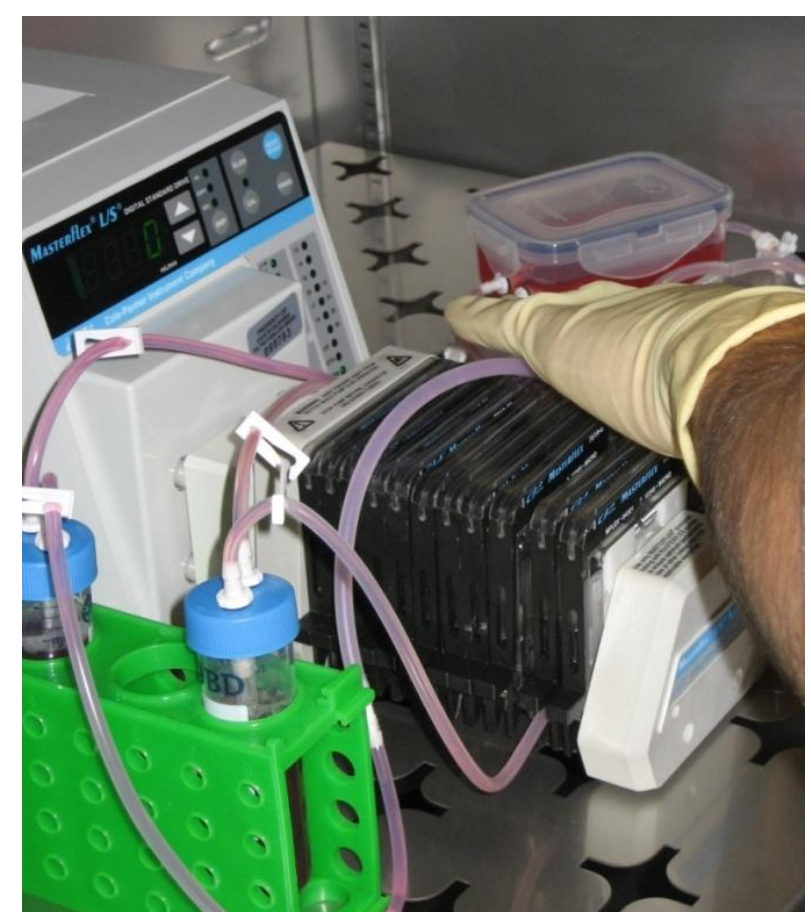

Figure 3.30- Attachment of the Ideal BVM bioreactor system to a peristaltic pump. The bioreactor system functioned properly without leakage.

The luminal inlet, luminal outlet and extra luminal outlet configuration permitted variation in flow path. Transluminal and transmural flow were easily controlled via the one-way stopcock attached to the luminal outlet. By closing off the luminal outlet path, through the use of the one-way stopcock, transmural flow through the porous ePTFE scaffold wall was created, with flow exiting the extra luminal outlet. Transmural flow was used for conditioning and cell sodding of the scaffold material. By opening the oneway stopcock, transluminal flow was restored through the luminal outlet. The one-way stopcock was easy to use and provided a quick and simple method for controlling the flow path. The lever was easy to turn and once in position flow was immediately and firmly blocked. The merging of the luminal outlet and extra luminal outlet into one main 
outlet via the Y-connector made attachment to the two-port media reservoir simple with the need for only one connection. The three-way stopcock attached to the luminal inlet easily functioned as both a flow path and cell sodding port. With two female luer thread fittings, the three-way stopcock allowed the Ideal BVM system to be connected to the two-port media reservoir as well as maintain an extra port for cell sodding and media injection.

Two-port media reservoirs were used in conjunction with the Ideal BVM system and also functioned properly without any leakage. Liquid was pulled from the media reservoir through the short outlet tube extending into the media and pumped through the bioreactor system via a peristaltic pump. Liquid drops could be seen falling from the inlet port, allowing for quick and easy visualization of flow as well as removal of air bubbles. Media reservoirs were EO sterilized prior to use. A photo of the media reservoir setup is shown below in Figure 3.31. 


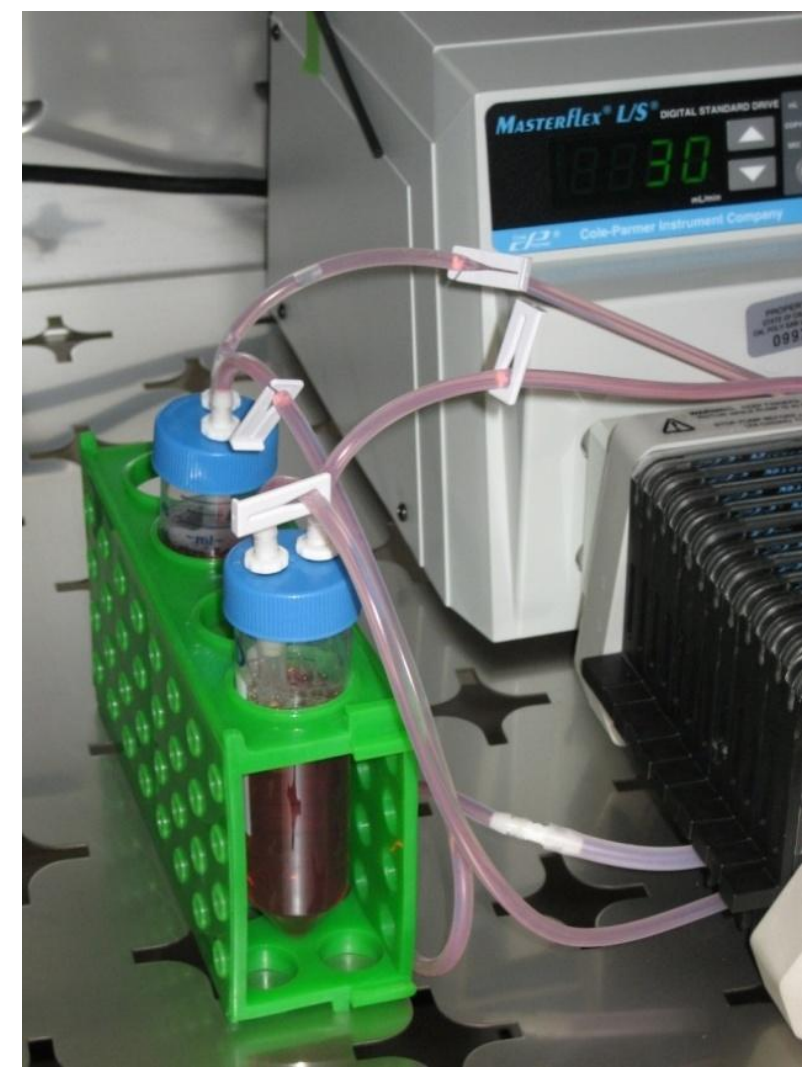

Figure 3.31- Two-port media reservoirs in use. The two-port media reservoirs functioned properly without leakage. Flow was established via a peristaltic pump.

\section{Comparison of Ideal BVM System to Original BVM System}

The Ideal BVM system and original BVM system were setup side-by-side by a lab member and a user feedback survey was completed which compared the two systems. Figures 3.32 and 3.33 below show the two systems being setup side-by-side and in the incubator connected to a peristaltic pump. The criteria evaluated and the results of the user feedback survey are summarized in Table 3.4. The completed user feedback survey can be found in Appendix F. 


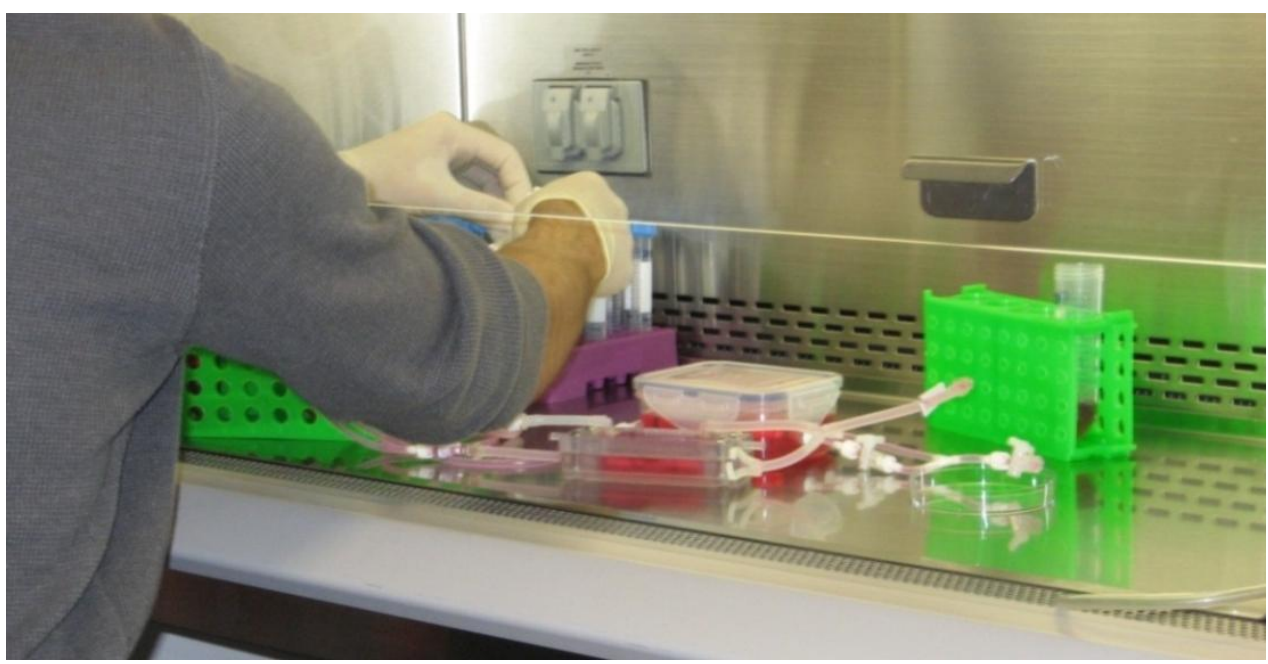

Figure 3.32- Side-by-side setup of original BVM and Ideal BVM systems. A lab member performed a sterile setup of both systems.

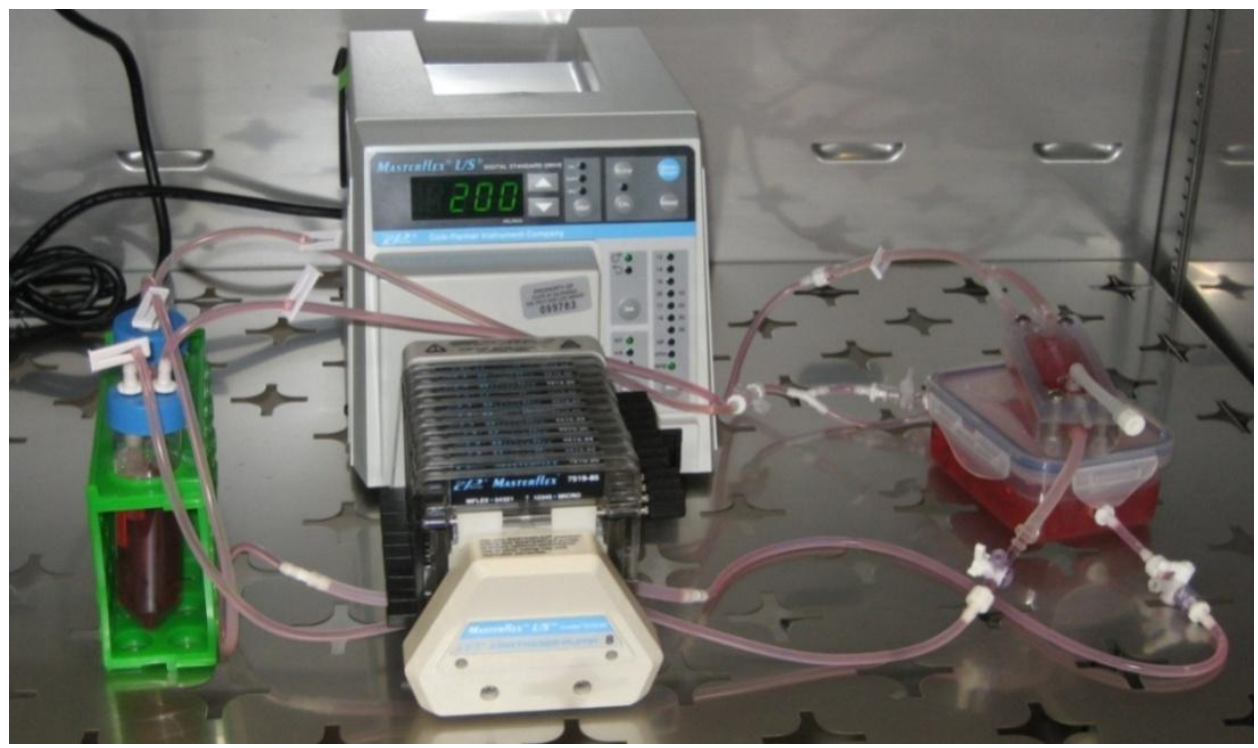

Figure 3.33- Side-by-side setup of original BVM and Ideal BVM bioreactor systems. Both systems were setup in the incubator and connected to a peristaltic pump to create flow. 


\begin{tabular}{|c|c|c|c|c|c|c|c|c|c|}
\hline \multirow{3}{*}{ Overall Usability } & \multicolumn{4}{|c|}{ Original BVM System } & \multicolumn{5}{|c|}{ Ideal BVM System } \\
\hline & \multicolumn{2}{|c|}{ Difficult } & \multicolumn{2}{|c|}{ Easy } & \multicolumn{2}{|c|}{ Difficult } & \multicolumn{3}{|c|}{ Easy } \\
\hline & 1 & 23 & 4 & 5 & 1 & 2 & 3 & 4 & 5 \\
\hline Setup & 1 & $2 B$ & 4 & 5 & 1 & 2 & 3 & 4 & 5 \\
\hline Scaffold Assembly & 1 & 23 & 五 & 5 & 1 & 2 & 3 & 4 & 5 \\
\hline Scaffold Insertion & 1 & $2 \longdiv { 3 }$ & 4 & 5 & 1 & 2 & 3 & 4 & 5 \\
\hline Media Injection & 1 & 23 & 4 & 5 & 1 & 2 & 3 & 4 & 5 \\
\hline Seal-Ability & 1 & $2 \longdiv { 3 }$ & 4 & 5 & 1 & 2 & 3 & 4 & 5 \\
\hline Aseptic Use & 1 & $2 \sqrt[3]{2}$ & 4 & 5 & 1 & 2 & 3 & 4 & 5 \\
\hline Bioreactor Assembly & 1 & 23 & 4 & 5 & 1 & 2 & 3 & 4 & 5 \\
\hline Control of Flow & 1 & $2 \sqrt[3]{2}$ & 4 & 5 & 1 & 2 & 3 & ๑ & 5 \\
\hline \multirow[t]{2}{*}{ Adjustability } & 1 & 23 & 4 & 5 & 1 & 2 & 3 & 母 & 5 \\
\hline & \multicolumn{4}{|c|}{ Awkward Convenient } & \multicolumn{2}{|c|}{ Awkward } & \multicolumn{3}{|c|}{ Convenient } \\
\hline \multirow[t]{2}{*}{ Components and Fittings } & 1 & 23 & 4 & 5 & 1 & 2 & 3 & 4 & 5 \\
\hline & Poor & & Excel & ellent & Poo & & & Exce & lent \\
\hline Component Configuration & 1 & 23 & 4 & 5 & 1 & 2 & 3 & 4 & 5 \\
\hline
\end{tabular}

Table 3.4- Summary of user feedback survey. The Ideal BVM system and original BVM system were compared based on the criteria listed. 
Based on the user feedback survey comments one of the advantages of the Ideal BVM system was that the chamber lid can be sterilized separately from the rest of the BVM chamber. In addition, the lid is not permanently attached to BVM chamber, and does not need to be held open, freeing up a second hand for use during scaffold insertion and work in the hood. Both these features were helpful in maintaining sterility and made setup easier. Another noted advantage was that the four snapping latches of the lid eliminated the need for tools when closing and sealing the BVM chamber. The Ideal BVM system also utilized stopcocks instead of side clamps to control flow and since the BVM chamber is not filled to the top with media the need to eliminate air bubbles during setup was no longer a challenge. Finally, with inlet and outlet fittings arranged so that only one configuration exists to setup the bioreactor system time was not wasted and there was no confusion when connecting the Ideal BVM system to the two-port media reservoir.

Based on the user feedback survey comments one of the disadvantages of the Ideal BVM system was that a larger volume of media was needed to fill the BVM chamber. Also, the larger container takes up more space in the incubator. However, it is important to note that the increased chamber volume only requires a one-time fill, as opposed to the reservoirs which are replaced every 3 days, and that these larger containers are stackable. The disadvantages, therefore, are offset by the advantages of the Ideal BVM system in comparison to the original BVM system. 


\section{Blood Vessel Development}

The Ideal BVM system successfully cultivated an in vitro blood vessel mimic by supporting cell sodding and cultivation. The scaffold was aseptically inserted and human umbilical artery smooth muscle cells (HUASMC) were pressure sodded onto the luminal surface of the ePTFE scaffold. Cells adhered to the scaffold surface and were distributed along the length of the vessel. Scanning electron microscope (SEM) images of an ePTFE scaffold surface are shown below in Figures 3.34 and 3.35. An SEM image of bare ePTFE, which represents the scaffold prior to cell sodding, is shown for reference followed by SEM images of the ePTFE with cells adhered to the surface.

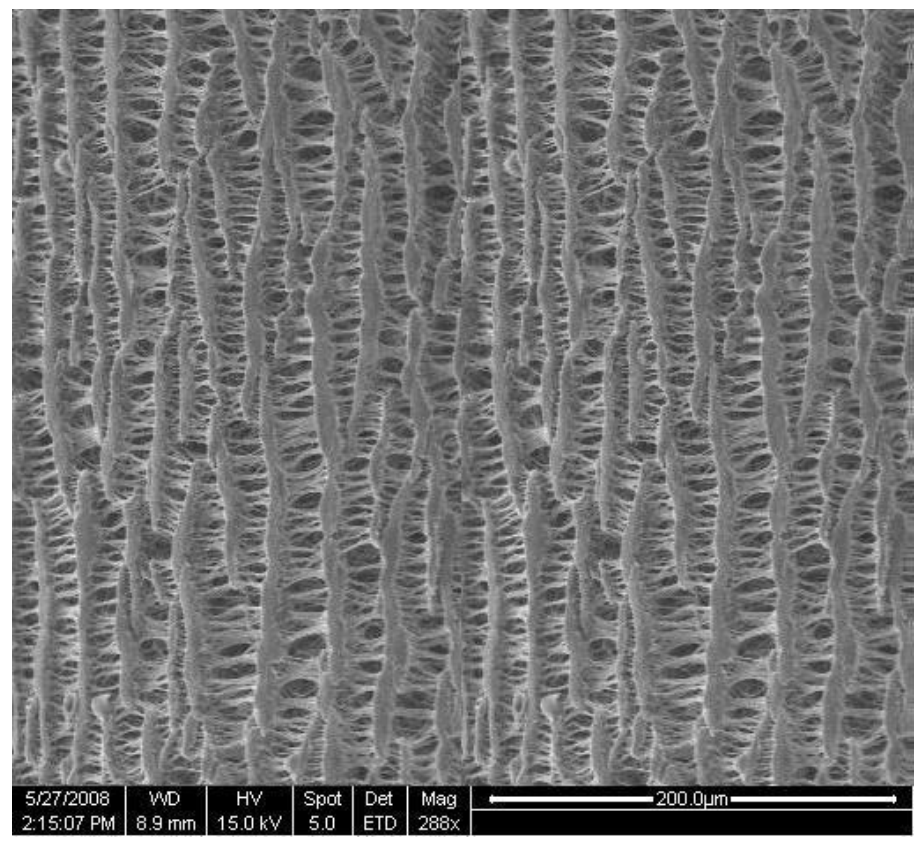

Figure 3.34- SEM image of bare ePTFE scaffold surface. 


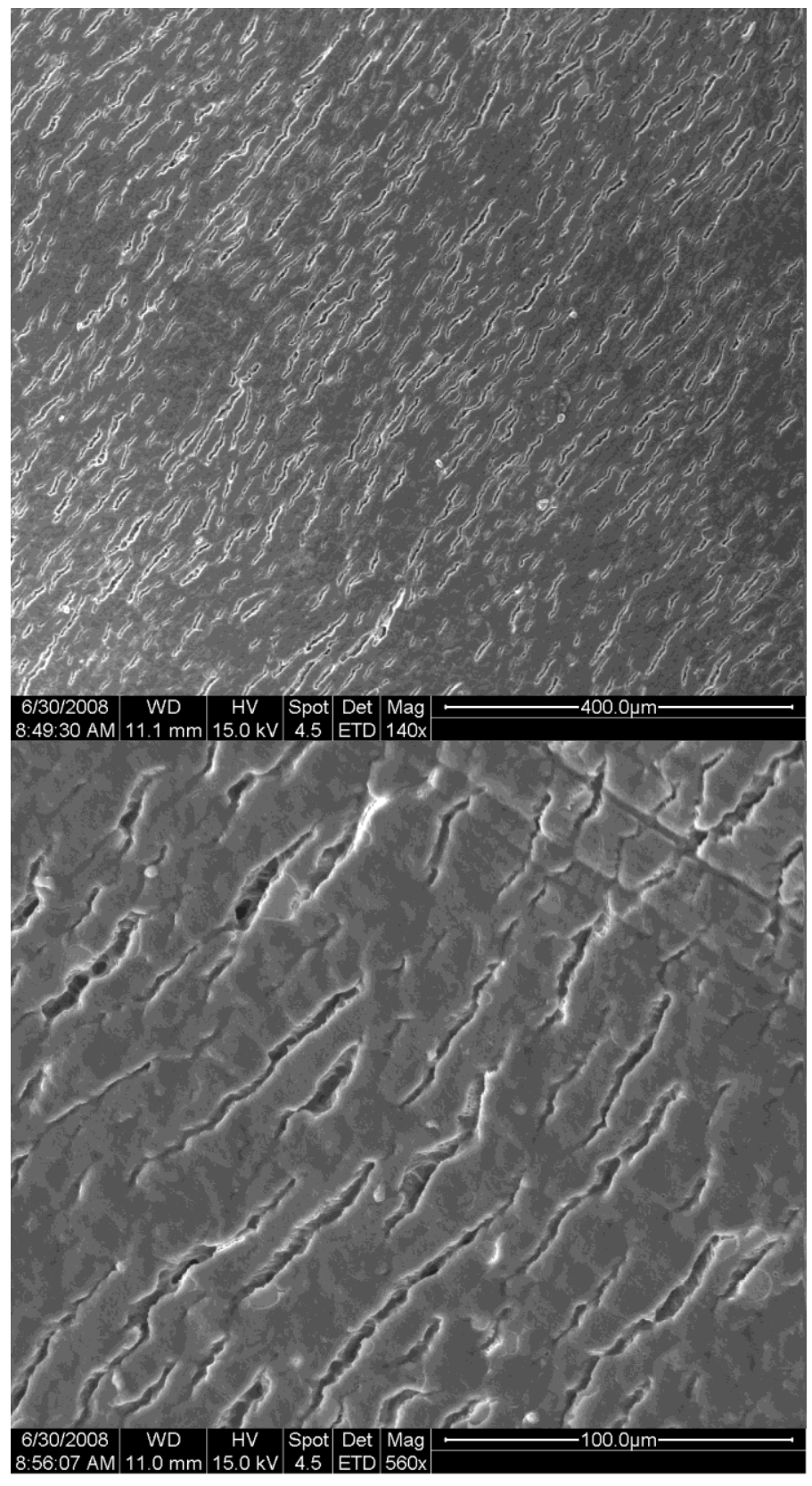

Figure 3.35- SEM images of ePTFE scaffold surface with cells. The Ideal BVM system successfully cultivated an in vitro blood vessel mimic with cell deposition and cell adherence to the scaffold surface. 


\section{Discussion and Conclusions}

The new Ideal BVM system and two-port media reservoirs were successfully built and implemented. The work described in this chapter supports the use of the Ideal BVM system for cultivation of in vitro blood vessel mimics. The finalized design and setup procedures were described in detail with testing performed to validate and verify the Ideal BVM bioreactor system functionality and use. The Ideal BVM incorporated a user friendly design and assembly was simple and straightforward. The scaffold was easy to insert without the need for bending or twisting. The leak-proof chamber was easy to seal and permitted quick and sterile assembly in the hood. Scaffold length and diameter were adjustable for greater testing variability. The chamber was optically clear allowing for easy visibility of scaffold, media and stent placement and was sterilizable by ethylene oxide (EO) gas. The Ideal BVM chamber was easy to manufacture without the need for adhesive or special tooling and was constructed of materials and components that were readily available and easy to obtain resulting in a low cost design that was easy to scale up. Multiple chambers could be configured in the incubator and the use of biocompatible materials allowed cells to grow and function.

These findings support the hypothesis that design changes could be made and new configurations implemented to the original BVM system that would improve function and usability for preclinical device testing. Specifically, the work described in this chapter supports the hypothesis that a BVM chamber could be created which has the advantages of easy graft insertion and seal-ability, as well as the ability to accommodate 
varying sizes of vessel scaffolds, all while maintaining the needs of a tissue engineering bioreactor system.

Several other bioreactor systems currently exist for development of tissue engineered blood vessels (TEBV). Most of these systems, similar to the Ideal BVM system, use a peristaltic pump to create flow within an optically clear chamber. However, chamber design, scaffold orientation, and general configuration vary considerably. For example, a custom designed vertical bioreactor chamber was built which housed a noncompliant silicone scaffold sodded with endothelial cells (EC) to examine the effects of shear stress and intravascular pressure (Punchard, et al. 2007). A second design utilized a large laboratory flask with sodding and cultivation of vascular smooth muscle cells (VSMC) on a PLCL [poly-(lactide-co-caprolactone)] scaffold oriented vertically within the chamber (Jeong, et al. 2005). These vertical bioreactor designs are shown below in Figure 3.36. Two other designs which examined the effects of pulsatile flow on smooth muscle and endothelial cell development utilized bioreactor systems that were very similar to the Ideal BVM system, with horizontal scaffold orientation (Hoerstrup, et al. 2001; Engbers-Buijtenhuijs, et al. 2006). These systems were assembled so that many scaffolds could be cultivated at once. Figure 3.37 shows the basic schematics of these two horizontal systems. In comparison with these bioreactor systems, the Ideal BVM differed in that it was designed for the purpose of preclinical testing. Therefore, separate individual flow paths were needed for each system and the ability to scale-up was a large focus. However, in general, each bioreactor system for the development of tissue engineered blood vessels must be designed so that sterility is maintained at all times and flow can be created through a scaffold lumen. Designs differ based on the available 
resources, desired features, purpose of the study, and designer ingenuity. In the case of the Ideal BVM system specific criteria were set in advance which guided the overall design.
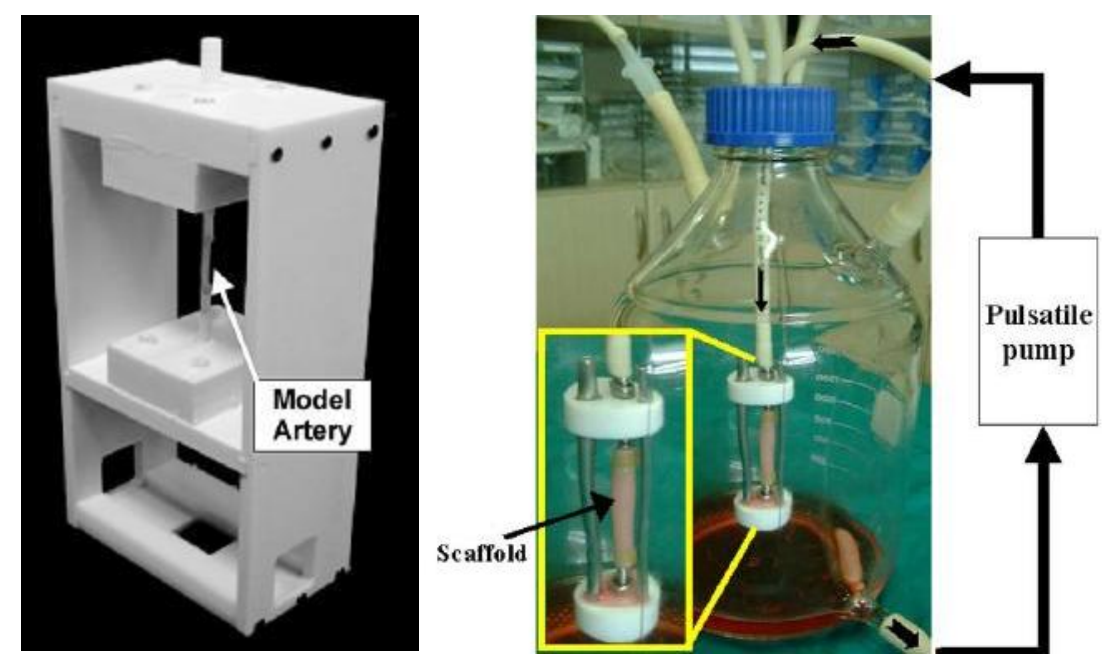

Figure 3.36- Vertical bioreactor chambers.

(Permission granted to reprint image from Punchard, et al. 2007 published in Journal of Biomechanics by Elsevier Limited.; Permission granted to reprint image from Jeong, et al. 2005 published in Biomaterials by Elsevier Limited.; see Appendix G) 

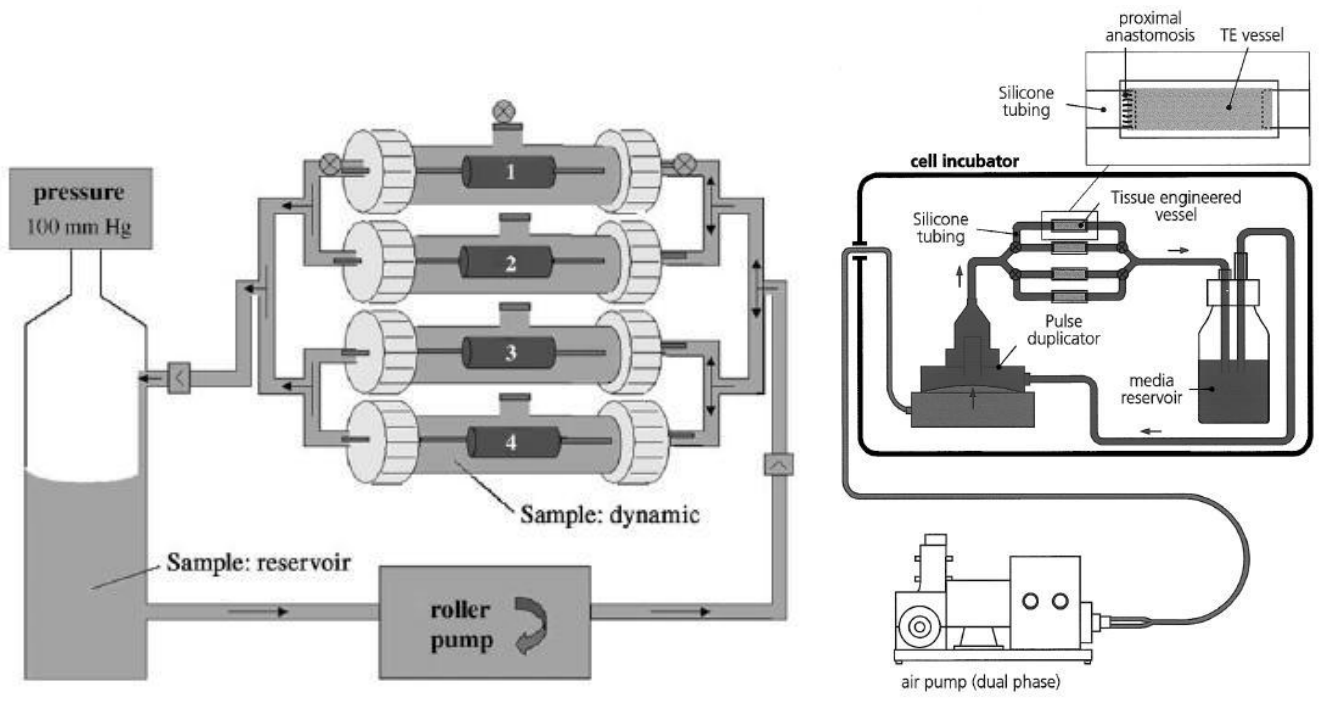

Figure 3.37- Horizontal bioreactor systems.

(Permission granted to reprint image from Hoerstrup, et al. 2001 published in European Journal of Cardio-Thoracic Surgery by Elsevier Limited.; Permission granted to reprint image from Engbers-Buijtenhuijs, et al. 2006 published in Biomaterials by Elsevier Limited.; see Appendix G)

An additional advantage of the Ideal BVM system is that the basic design used to create the inlet and outlets of both the Ideal BVM chamber and two-port media reservoir can be easily applied to a wide range of container shapes and sizes. Depending on need and desired scaffold configuration holes can be placed in any location within the container. For example, a larger container could allow for longer scaffold lengths, or placement of inlet and outlet ports in varying configurations could accommodate more physiologic scaffold orientations, such as bends and bifurcations. Also, if additional ports are desired for injection of media into the space surrounding the scaffold, they can be easily added at any location desired. It is important to note that as container length and 
size increase so does container volume. Volume of the BVM chamber is not a critical factor, but does affect the amount of media needed to fill the system and should be considered prior to implementing a different container size.

Given the possible variability of the general Ideal BVM chamber design, the following chapter will explore the potential to create alternate chambers for the evaluation of more complex vascular devices as well as the creation of chambers that accommodate more physiological vascular models. This idea of next generation BVM systems will be explored as potential future uses of the in vitro blood vessel mimic for preclinical device testing. 


\section{DESIGN OF NEXT GENERATION BVMs}

Introduction

A BVM system can allow initial assessments to be performed on a large number of coating combinations and stent configurations in order to direct animal and clinical studies toward the most promising devices. The aim of the work in the previous chapters was to improve upon the original BVM system by developing a new, more ideal design and to describe in detail the finalized design, construction and setup of the Ideal BVM system and the testing performed to validate and verify its functionality and use.

The design of both the original BVM and Ideal BVM systems focused on straight, simple scaffold orientations representing simple lesions and single vessel disease (Fournier, et al. 2005). Coronary stent implantation, however, is being performed in an increasing number of patients with a wide spectrum of clinical and lesion characteristics. The anatomical characteristics of coronary arteries vary considerably, ranging from straight simple geometries to extremely angulated bends and curves as well as bifurcations and long lesions (Ellis, et al. 1990; Zhu, et al. 2008; Smith, et al. 2001). Depending on the individual, the anatomical structure of the coronary arteries can include all or some of the characteristics identified above. These lesion characteristics, including long lesions, angulated geometries, and bifurcations, will be described in detail below and will provide an introduction to the aims of this chapter.

The Ideal BVM system was designed to test stents ranging from 2.0-5.0 mm diameters and 8-38 mm lengths (Kandzari, Tcheng and Zidar 2002; Kraitzer, Kloog and Zilberman 2008), in order to accommodate the variations of coronary arteries observed in 
clinical practice and the stents currently available in the market. However, single coronary lesions are often longer than $38 \mathrm{~mm}$, sometimes greater than $60 \mathrm{~mm}$ in length, and treatment requires multiple overlapping stents (Ruchin, et al. 2008). Using the BVM system for preclinical testing of overlapping stents could allow for better understanding of the cellular response at the overlapping junctions as well as the effect of the potentially double local drug does when using drug-eluting stents (Kang, et al. 2007). In addition, new interlocking stent designs, such as the Custom NX(R) DES System manufactured by XTENT, Inc., are exploring the possibility of deploying stents up to $60 \mathrm{~mm}$ in total length (Stella, et al. 2008). Therefore, a BVM chamber for the preclinical testing of overlapping and longer total stent lengths would be beneficial.

According to the American College of Cardiology/American Heart Association (ACC/AHA) Guidelines for Percutaneous Coronary Intervention, non-angulated coronary artery geometries are defined as bends that are less than $45^{\circ}$, while moderately angulated geometries represents bends between $45^{\circ}$ and $90^{\circ}$, and extremely angulated geometries are considered bends in excess of $90^{\circ}$ (Smith, et al. 2001). A variety of angled geometries are illustrated below in Figure 4.1. Figure 4.2 is an X-ray angiogram image of the coronary arteries and shows the variation of curvatures observed in clinical practice. 


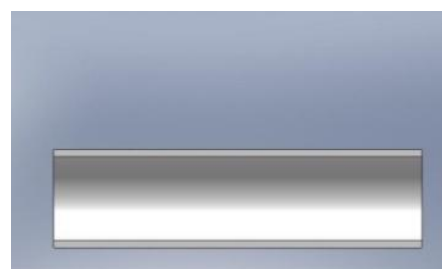

a)

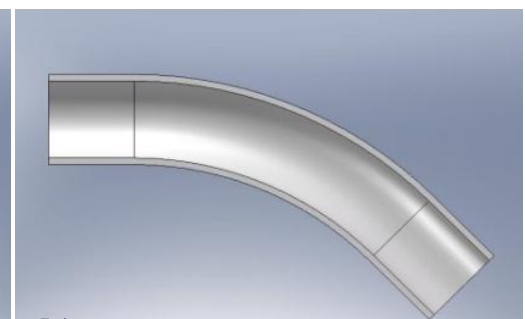

b)

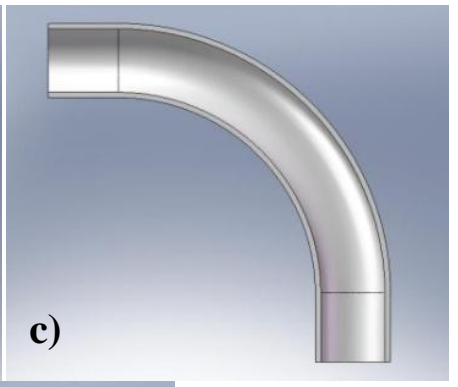

d)

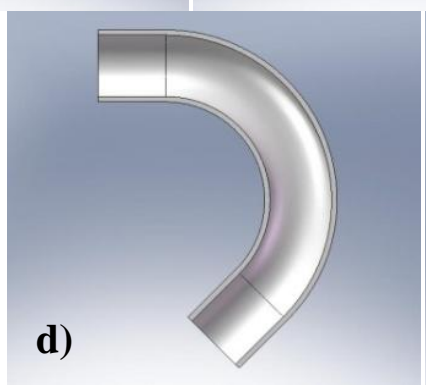

e)

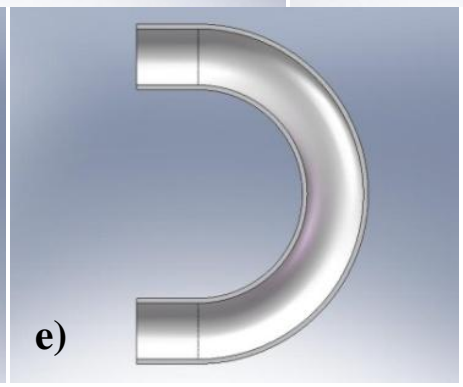

Figure 4.1- Coronary artery geometries. Non-angulated bends are between $0^{\circ}$ (a) and $45^{\circ}$ (b), moderately angulated bends are between $45^{\circ}$ and $90^{\circ}$ (c), and extremely angulated geometries are greater than $90^{\circ}$. Examples of extremely angulated geometries are shown as $135^{\circ}(\mathrm{d})$ and $180^{\circ}(\mathrm{e})$. 


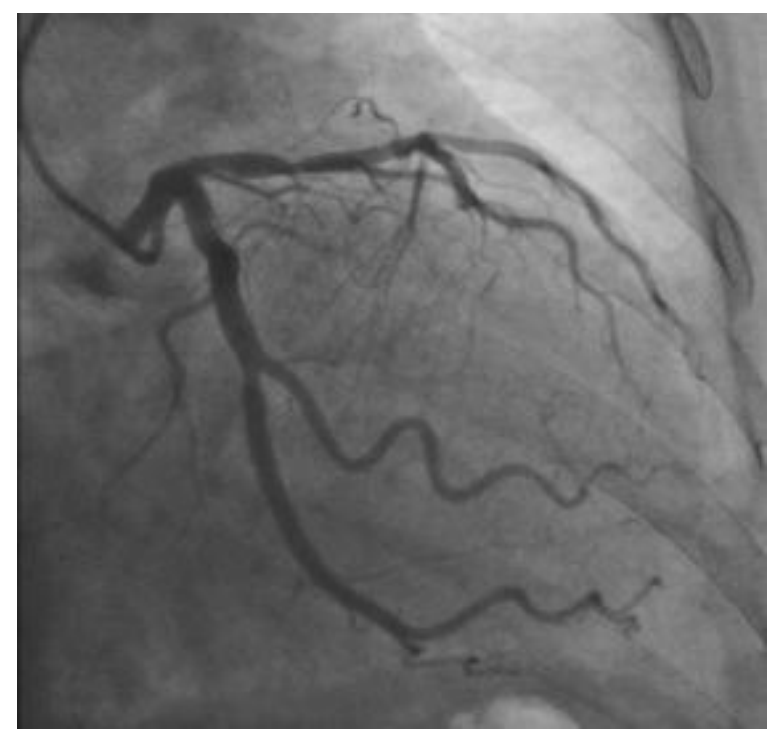

Figure 4.2- X-ray angiogram image of the coronary arteries. Various angled bends and curvatures are present.

(Permission granted to reprint image from Xu, et al. 2007 published in Computer Methods and Programs in Biomedicine by Elsevier Limited.; see Appendix G)

As mentioned previously, the Ideal BVM system was designed for straight, simple scaffold orientations representing simple lesions and ideal anatomical geometry. In clinical practice, however, coronary arteries are not straight and uniform. They run along the surface of the heart and are curved, bent, and angulated (Smith, et al. 2001). In addition, it has been observed that plaque accumulation is related to vessel curvature, with plaque accumulating more rapidly in angulated vessels where wall shear stress is low (Wahle, et al. 2006; Zhu, et al. 2008). As a result, stents are most often placed in curved positions within the coronary arteries and remain in a bent position throughout their lifetime (Fournier, et al. 2005; Smith, et al. 2001). Many coronary stents, such as the MULTI-LINK manufactured by Abbott Vascular, CYPHER ${ }^{\circledR}$ manufactured by Cordis, 
and TAXUS ${ }^{\circledR}$ manufactured by Boston Scientific, also claim superior flexibility and conformity which allow the stent to mold to the natural contours of the vessel wall (Serruys and Gershlick 2005; Rieu, et al. 2003). Therefore, a BVM chamber for the preclinical testing of devices in curved and angulated vessels would be beneficial and would represent a more physiological blood vessel mimic model.

As described by the ACC/AHA a coronary bifurcation occurs when one main vessel branches out into two smaller vessels, one being the continuation of the main vessel, and the other referred to as the side branch (Smith, et al. 2001). A coronary bifurcation lesion is defined as a narrowing involving both the main vessel and a significant side branch (Louvard, et al. 2008). Illustrations of two bifurcated geometries, Y-shaped and T-shaped, are shown below in Figure 4.3. Figure 4.4 is an X-ray angiogram image of Y-shaped and T-shaped bifurcations observed in clinical practice.

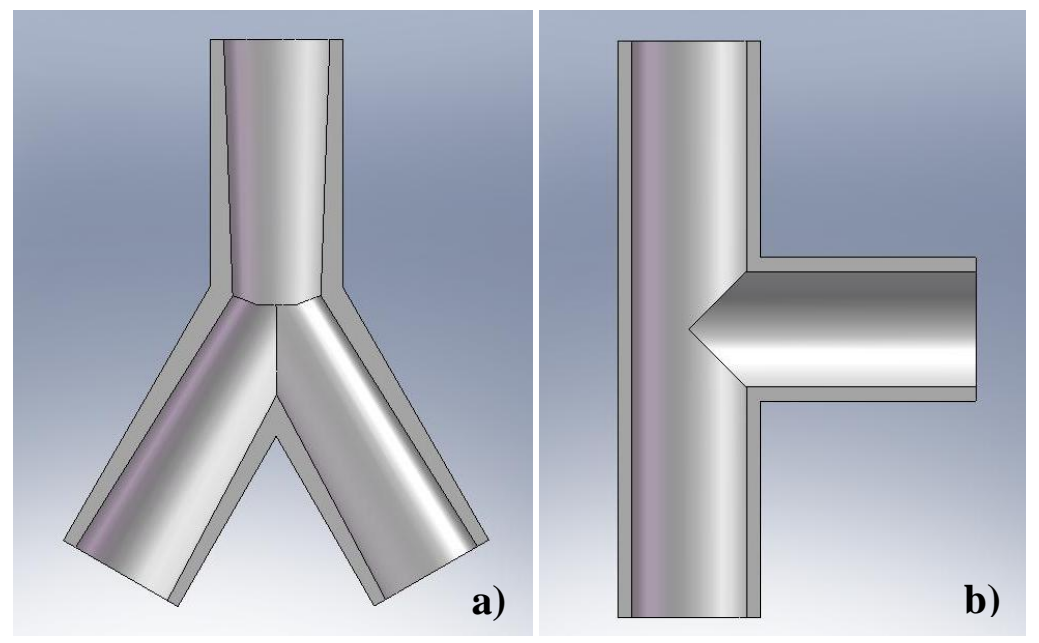

Figure 4.3- Bifurcated coronary artery geometries. Y-shaped (a) and T-shaped (b). 


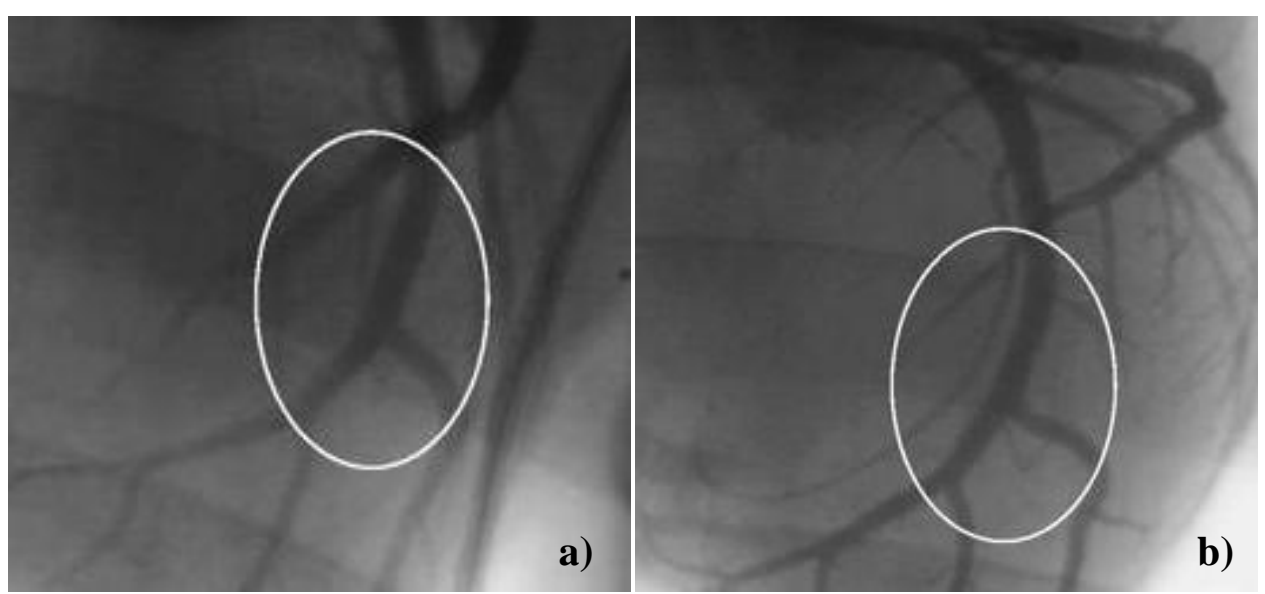

Figure 4.4- X-ray angiogram image of bifurcated coronary arteries.

Y-shaped (a) and T-shaped (b).

(Permission granted to reprint image from Ikeno, Buchbinder and Yeung 2007 published in Cardiovascular Revascularization Medicine by Elsevier Limited.; see Appendix G)

Bifurcation lesions account for $15-18 \%$ of all coronary lesions and represent one of the most complex coronary lesions to treat since the lumen of both the main vessel and side branch are affected and require treatment (Sukhija, Mehta and Sachdeva 2008). Recent advances have allowed physicians to treat bifurcation lesions, where both the main vessel and side branch need to be restored. Many techniques, with the use of one or multiple stents, have been developed to treat bifurcation lesions (Sukhija, Mehta and Sachdeva 2008; Iakovou, Ge and Colombo 2005). The simplest approach is to stent the main vessel and dilate the side branch through the stent struts. A second approach is to use multiple stents and can be accomplished through a number of different techniques which were discussed in Chapter 1: V-stenting, simultaneous kissing, crush, T-stenting, modified T-stenting, Y-stenting, and skirt. Most recently, novel stents and delivery 
systems such as AXXESS ${ }^{\text {TM }}$ manufactured by Devax, Inc., PETAL ${ }^{\text {TM }}$ manufactured by Boston Scientific, and MULTI-LINK Frontier manufactured by Abbott Vascular have been developed specifically to treat bifurcation lesions (Grube, et al. 2007; Ikeno, Buchbinder and Yeung 2007; Miyazawa, et al. 2007; Lefèvre, et al. 2005). Therefore, a BVM chamber for the preclinical testing of devices in bifurcation shaped vessels would be beneficial and would support advancing technology.

In summary, the variation and complexity of coronary artery disease and the advanced capabilities to detect and determine the extent and severity of coronary lesions has led to an increased interest in intravascular device innovation (Kini 2006). Stents are most often placed in vessels with angulated geometries and remain in bent positions throughout their lifetime (Fournier, et al. 2005; Smith, et al. 2001). Long lesions can require two overlapping stents (Ruchin, et al. 2008) and bifurcation lesions often require two stents at varying angles or the use of a novel stent specifically designed for a bifurcation (Sukhija, Mehta and Sachdeva 2008; Ikeno, Buchbinder and Yeung 2007). Thus, the aim of the work in this chapter was to expand the BVM concept to meet the need of future technologies that currently have not been adequately addressed in preclinical models, such as long, angulated and bifurcation lesions. The hypothesis was that the need to test stents specifically for long, angulated and bifurcation lesions can be potentially addressed by using modified BVM systems. Although full implementation and testing of these models is beyond the scope of this thesis, the current work will explore the development of new and innovative models for the evaluation of more complex lesions as well as the creation of chambers that accommodate more physiological vascular models and scaffold orientations. Therefore, this chapter will 
explore the creation of alternate chambers for the evaluation of more complex vascular devices as potential future uses of the in vitro blood vessel mimic for preclinical device testing. 
Next Generation BVM System Designs

BVM System for Long Vessels

A BVM system for long vessels can be created by using a longer chamber, such as the $800 \mathrm{ml}, 8 " \times 5 " \times 2 "$, semitransparent, polypropylene rectangular snap and seal leak-proof food container (Lock \& Lock P/N HPL816) and implementing the same design and construction described in the previous chapter and detailed in Appendix $\mathrm{C}$ for the assembly of the luminal inlet and luminal outlets. Locations of the luminal inlet and luminal outlets are identical to the Ideal BVM system. A chamber of this size can accommodate scaffold lengths up to $170 \mathrm{~mm}$ which would allow long or multiple stents to be placed and overlapped within the vessel lumen. It is important to note that the volume of this chamber is greater than that of the Ideal BVM system. Volume of the BVM chamber is not a critical factor, but does affect the amount of media needed to initially fill the system and should be considered prior to implementation. The BVM system for long vessels is modeled below in Figures 4.5 and 4.6.

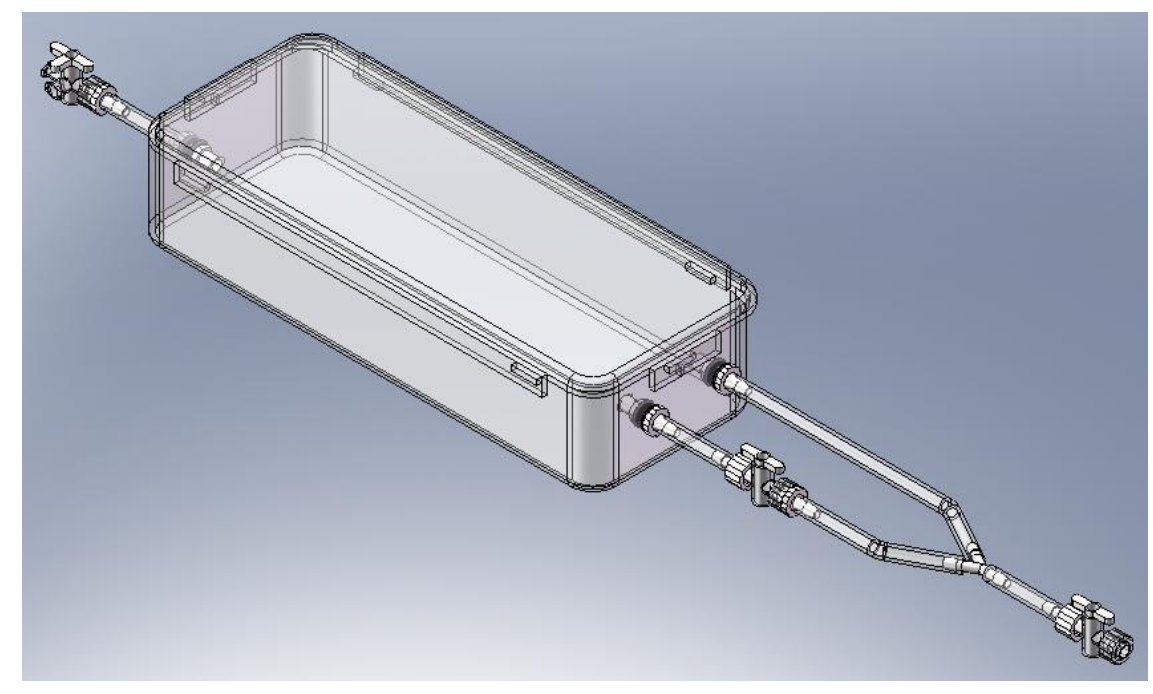

Figure 4.5- BVM system for long vessels. 


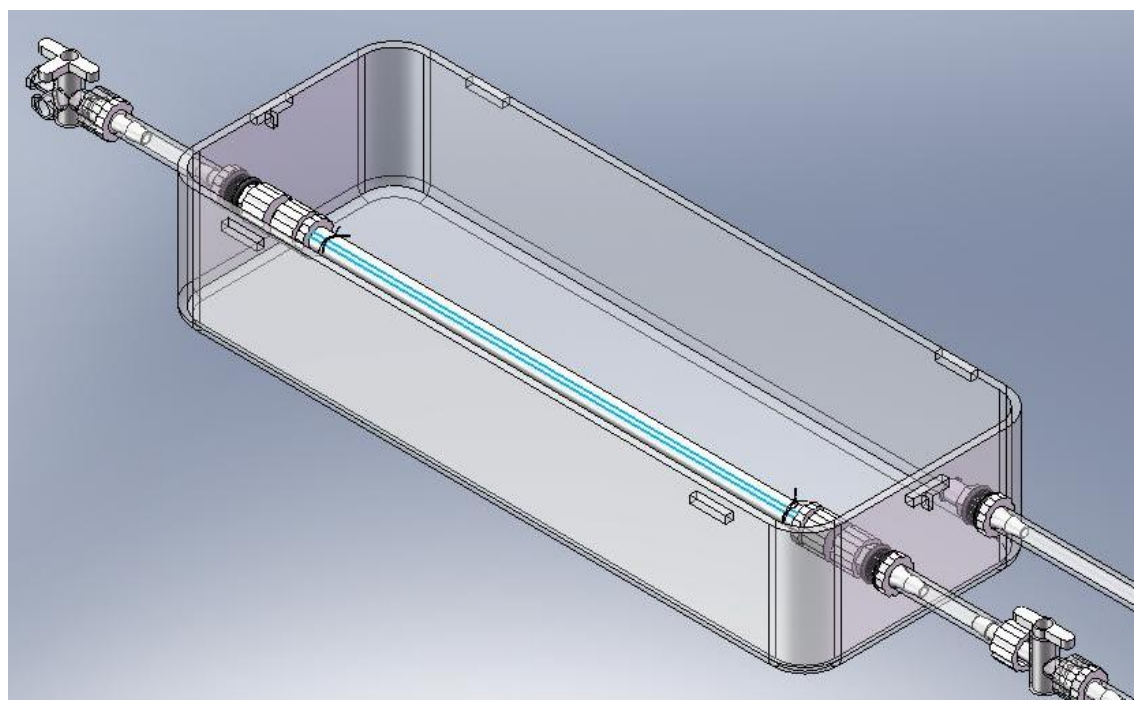

Figure 4.6- Scaffold setup for long vessels. Longer scaffold lengths can be accommodated in a modified BVM system by using a longer chamber.

Scaffold setup and insertion for the BVM system for long vessels will remain unchanged from the assembly process used for the Ideal BVM system described in the previous chapter, except for the length of ePTFE scaffold used. As in the previous design, male luer integral lock ring to female luer thread style couplers can be used to vary the overall scaffold length. A prototype of this model would be needed to establish the corresponding scaffold length versus number of couplers used.

The design of the BVM system for long vessels is almost identical to the Ideal BVM system and implements a chamber that is identical except for length and volume. As a result the BVM chamber for long vessels meets all of the design criteria established in the previous chapters. 
BVM System for Angulated Vessels

A BVM system for angulated and curved vessels can be created by varying the placement of the luminal inlet and luminal outlet holes on the Ideal BVM chamber. Construction of the luminal inlet and luminal outlet holes can be accomplished using the same materials and methods described in the previous chapter and detailed in Appendix C. Prototypes must be built in order to determine the exact dimensional location and placement of the luminal inlet and luminal outlet holes. However, the illustrations below approximate their general locations. A large variation of curves and angles can be created ranging from slight bends to $180^{\circ}$ turns, representing non-angulated, moderately angulated and severely angulated geometries. Construction of several modified BVM chambers for angulated vessels are described below. All designs utilize the same chamber as the Ideal BVM system, a $350 \mathrm{ml}, 4 "$ x 5" x 2", semitransparent, polypropylene rectangular snap and seal leak-proof food container (Lock \& Lock P/N HPL806).

The simplest bend that can be created requires only one change to the tubing configuration of the Ideal BVM system. Locations of the luminal inlet and luminal outlet holes are identical to the Ideal BVM system. By reversing the placement of the luminal outlet and extra luminal outlet and connecting the scaffold as described in the previous chapter, a slightly bent scaffold can be accommodated. This setup represents a nonangulated geometry and can accommodate scaffold orientations with bends of $45^{\circ}$ or less. The BVM system for slightly bent vessels is modeled below in Figures 4.7 and 4.8. 


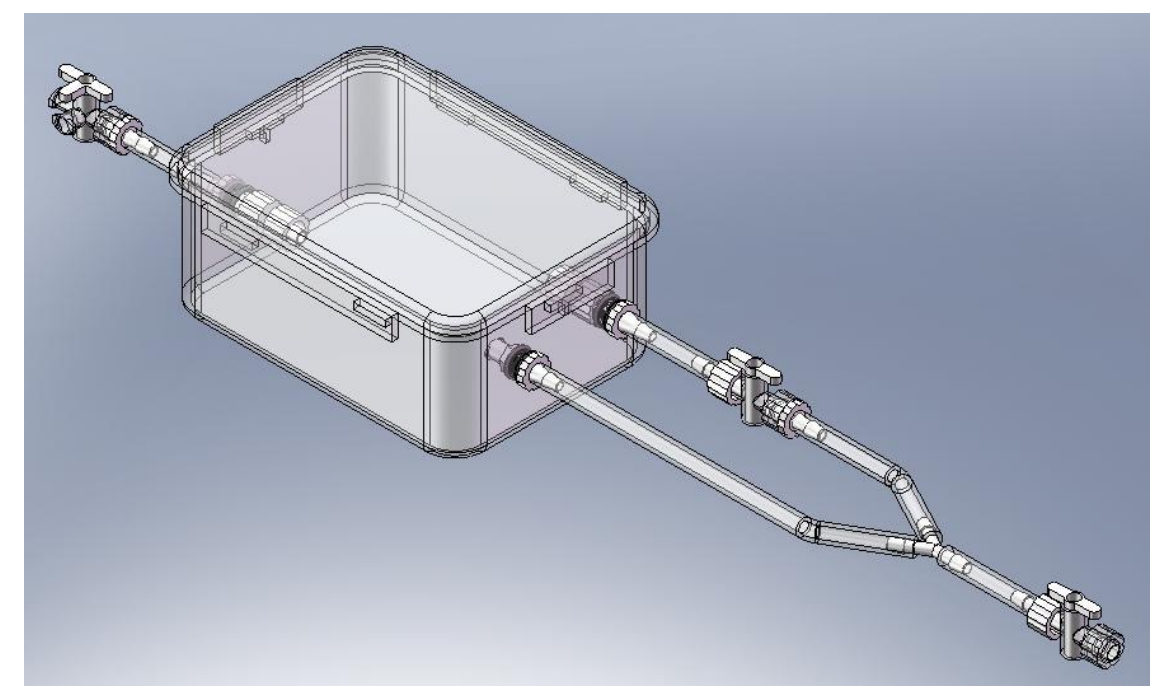

Figure 4.7- BVM system for slightly bent vessels.

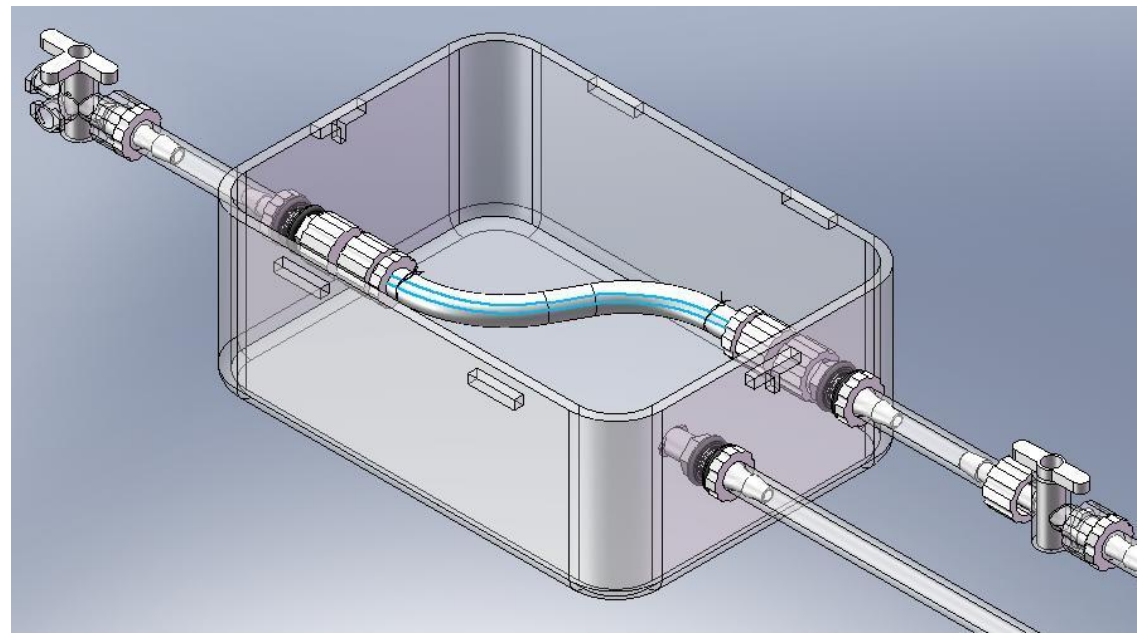

Figure 4.8- Scaffold setup for non-angulated vessels. A slightly bent scaffold can be accommodated in a modified BVM chamber by reversing the luminal outlet and extra luminal outlet positions.

The second bend that can be created requires only a slight modification to the configuration of the Ideal BVM system. By moving the luminal outlet and extra luminal outlet to the long side of the chamber and connecting the scaffold as described in the 
previous chapter, a moderately angled scaffold can be accommodated. This setup represents moderately angled geometry and can accommodate a scaffold orientation with a bend of $90^{\circ}$. The BVM system for moderately angled vessels is modeled below in Figures 4.9 and 4.10.

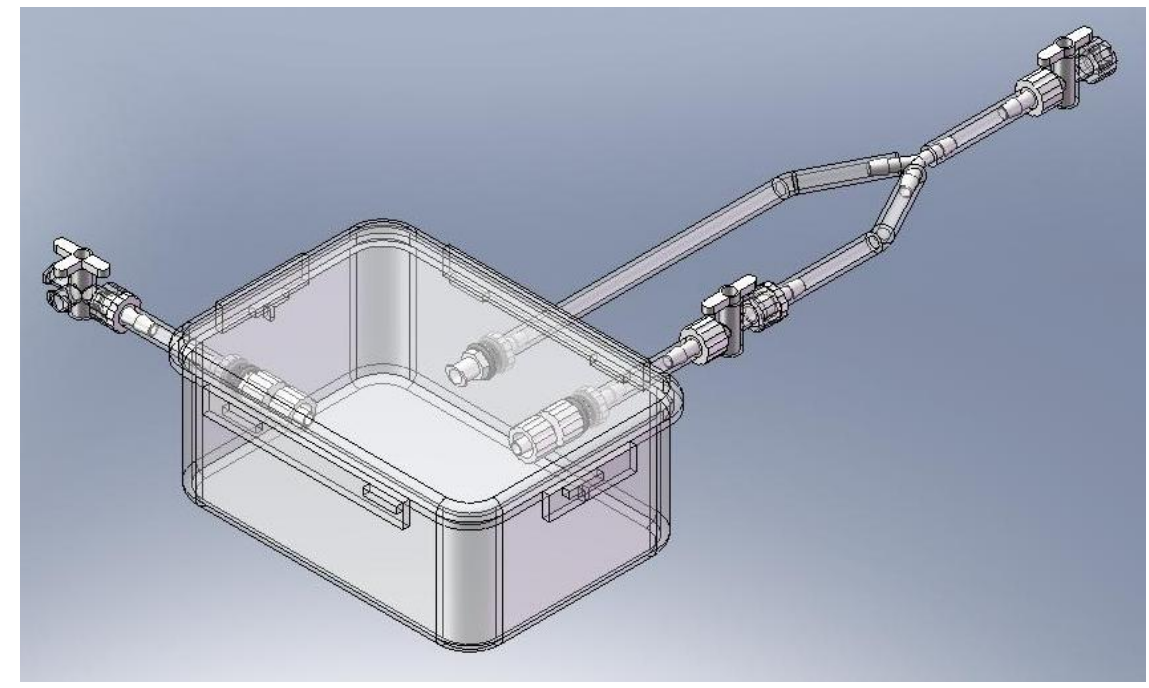

Figure 4.9- BVM system for moderately angled vessels.

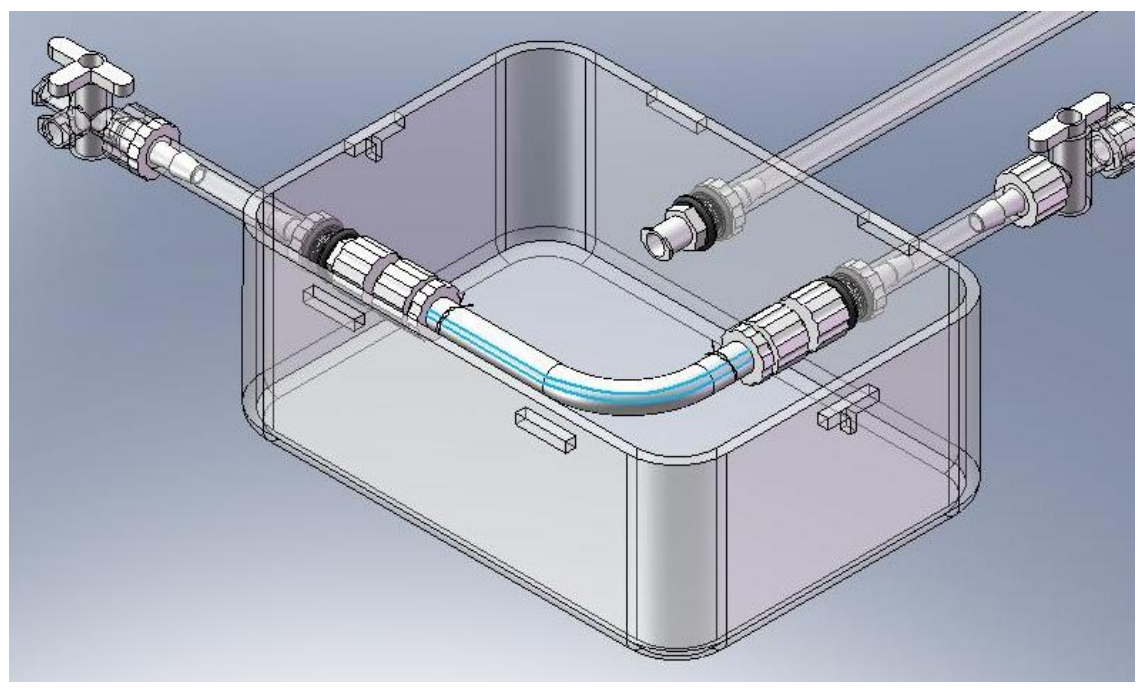

Figure 4.10- Scaffold setup for moderately angulated vessels. A moderately angled scaffold of $90^{\circ}$ can be accommodated in a modified BVM chamber by moving the luminal outlet and extra luminal outlet to the long side of the chamber. 
The third bend that can be created requires a minor modification to the configuration of the Ideal BVM system. By moving the luminal inlet, luminal outlet and extra luminal outlet to the long side of the chamber and connecting the scaffold as described in the previous chapter, a severely angled scaffold can be accommodated. This setup represents severely angled geometry and can accommodate a scaffold orientation with a bend of $180^{\circ}$. The BVM system for severely angled vessels is modeled below in Figures 4.11 and 4.12 .

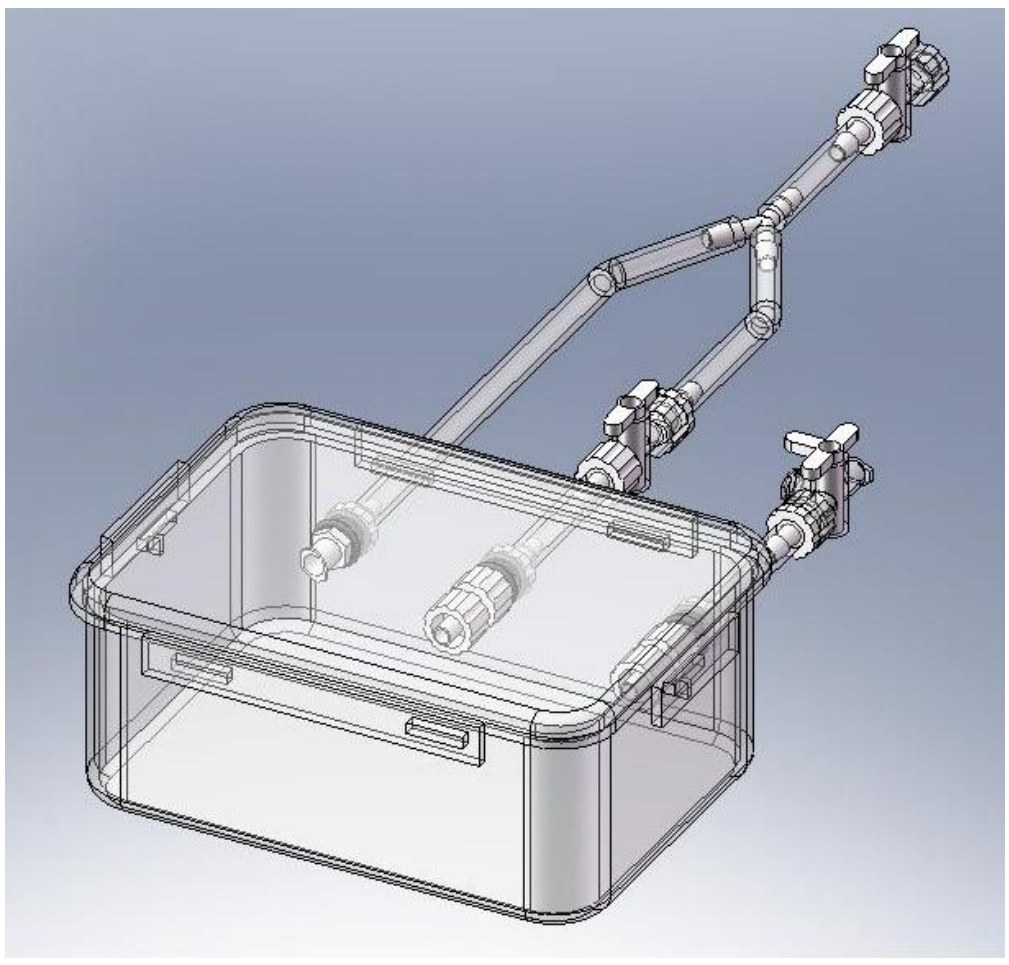

Figure 4.11- BVM system for severely angled vessels. 


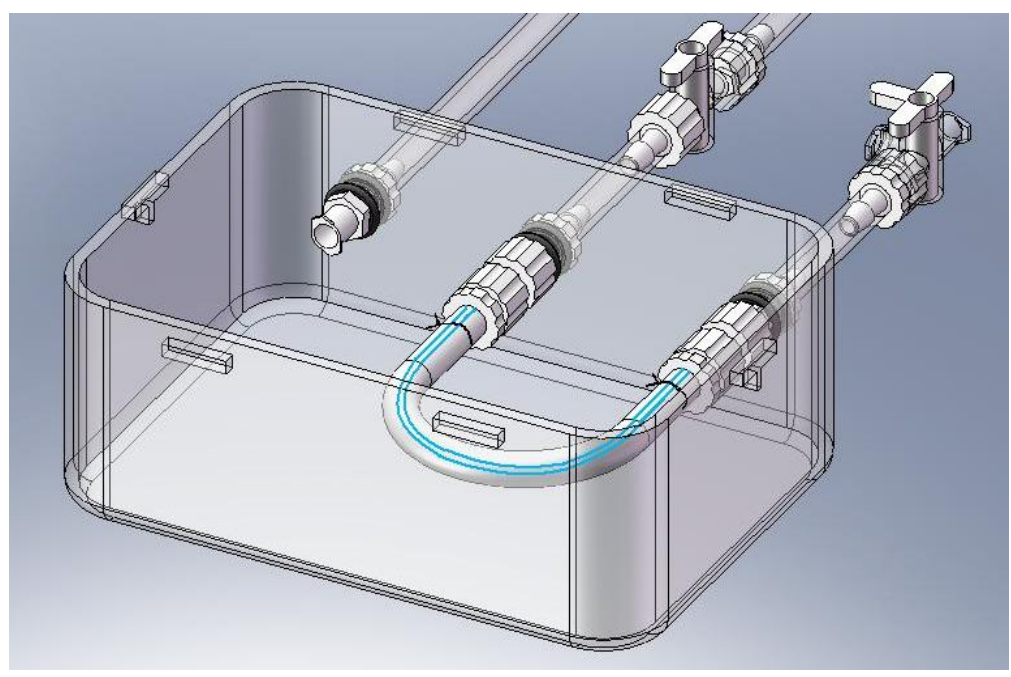

Figure 4.12- Scaffold setup for severely angulated vessels. A severely angled scaffold of $180^{\circ}$ can be accommodated in a modified BVM chamber by moving the luminal inlet, luminal outlet and extra luminal outlet to the long side of the chamber.

Scaffold setup and insertion for the BVM systems for angulated vessels will remain unchanged from the assembly process used for the Ideal BVM system described in the previous chapter, except for the lengths of ePTFE scaffolds used. As in the previous design, male luer integral lock ring to female luer thread style couplers can be used to vary the overall scaffold lengths in each design. Prototypes of each model are needed to establish the corresponding scaffold lengths versus number of couplers.

One challenge that may be encountered in these configurations is kinking and occlusion of the scaffolds due to their bent orientations. However, ePTFE material is flexible and compliant and once flow is created via a peristaltic pump, the force on the scaffold walls created by the pressure of the media may be strong enough to overcome any kinks or occluded bends that occur. This hypothesis will need to be verified and tested with full-scale prototypes. If kinking or occlusion proves to be a challenge a 
change in the scaffold material or fabrication method may need to be addressed. Preforming the scaffold in a bent orientation by using a mold or customized model could provide a solution. A thin wire coiled around the outside of the ePTFE could also be used to control the curvature and orientation without disrupting the inner lumen.

The designs of the BVM systems for angulated vessels are almost identical to the Ideal BVM system and use the exact same chamber. As a result the BVM chambers for angulated vessels meet all of the design criteria established in the previous chapters.

BVM System for Bifurcation Vessels

A BVM system for bifurcation vessels can be created by varying the placement of the luminal inlet, luminal outlet, and extra luminal outlet holes and adding a second luminal outlet to accommodate the side branch lumen. Construction of the luminal inlet and luminal outlet holes can be accomplished using the same materials and methods described in the previous chapter and detailed in Appendix C. Prototypes must be built in order to determine the exact dimensional location and placement of the luminal inlet and luminal outlet holes. However, the illustrations below approximate their general locations. The most notable change is the modified tubing configuration which is necessary to accommodate flow through the second luminal outlet. Construction of two modified BVM chambers for Y-shaped and T-shaped bifurcation vessels are described below. Each design utilizes the same chamber as the Ideal BVM system, a $350 \mathrm{ml}, 4$ " x $5 "$ x 2", semitransparent, polypropylene rectangular snap and seal leak-proof food container (Lock \& Lock P/N HPL806). 
The first bifurcation geometry that can be created requires some modifications to the configuration of the Ideal BVM system. By moving the extra luminal outlet to the long side of the chamber and adding a second luminal outlet in its place a Y-shaped bifurcation scaffold can be accommodated. A Y-shaped bifurcation scaffold can be setup and connected as described in the previous chapter with the addition of one female luer thread style to 200 series barb, 1/8" ID tubing (Value Plastics P/N FTLL230-1). One male-to-male luer lock connector, with one rotating collar (Qosina P/N 17644) also needs to be attached to the second outlet to hold the scaffold. The easiest way to configure the tubing is to simply add a second Y-connector with 200 series barbs, 1/8" ID tubing (Value Plastics P/N Y230-1) prior to the attachment of the one-way stopcock. In addition, a second one-way stopcock (Value Plastics P/N VP455980) must be added to the second luminal outlet to control flow. This modified orientation represents Y-shaped bifurcation geometry and can accommodate a Y-shaped scaffold. The BVM system for Y-shaped bifurcation vessels is modeled below in Figures 4.13 and 4.14.

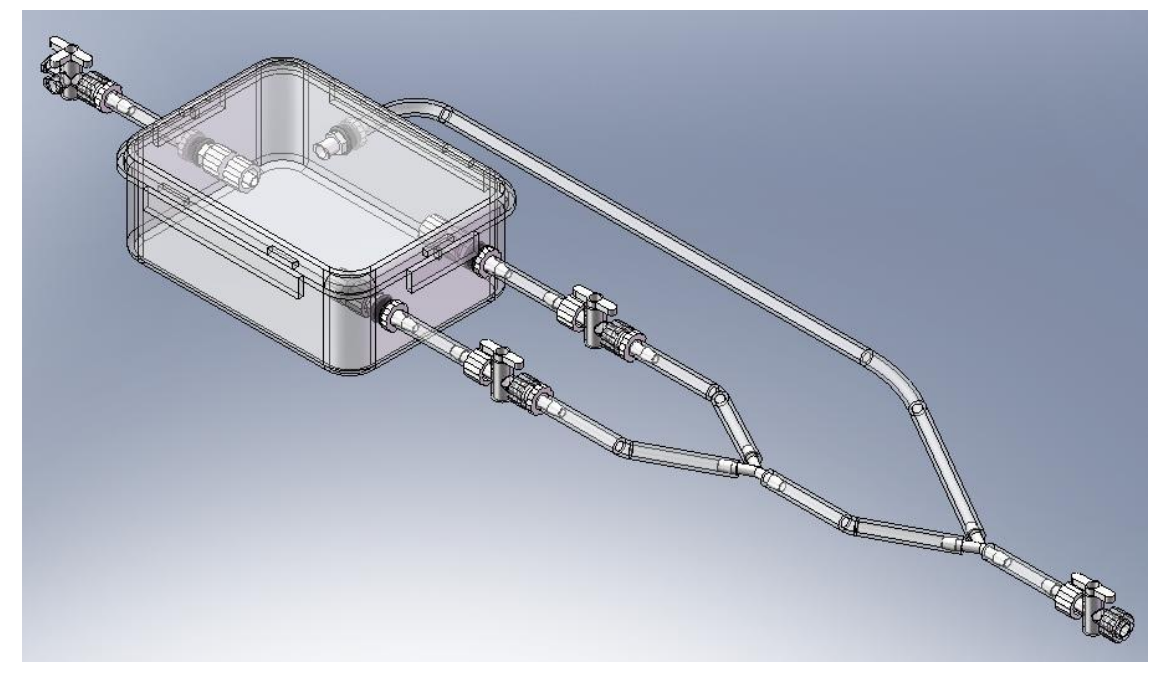

Figure 4.13- BVM system for Y-shaped bifurcation vessels. 


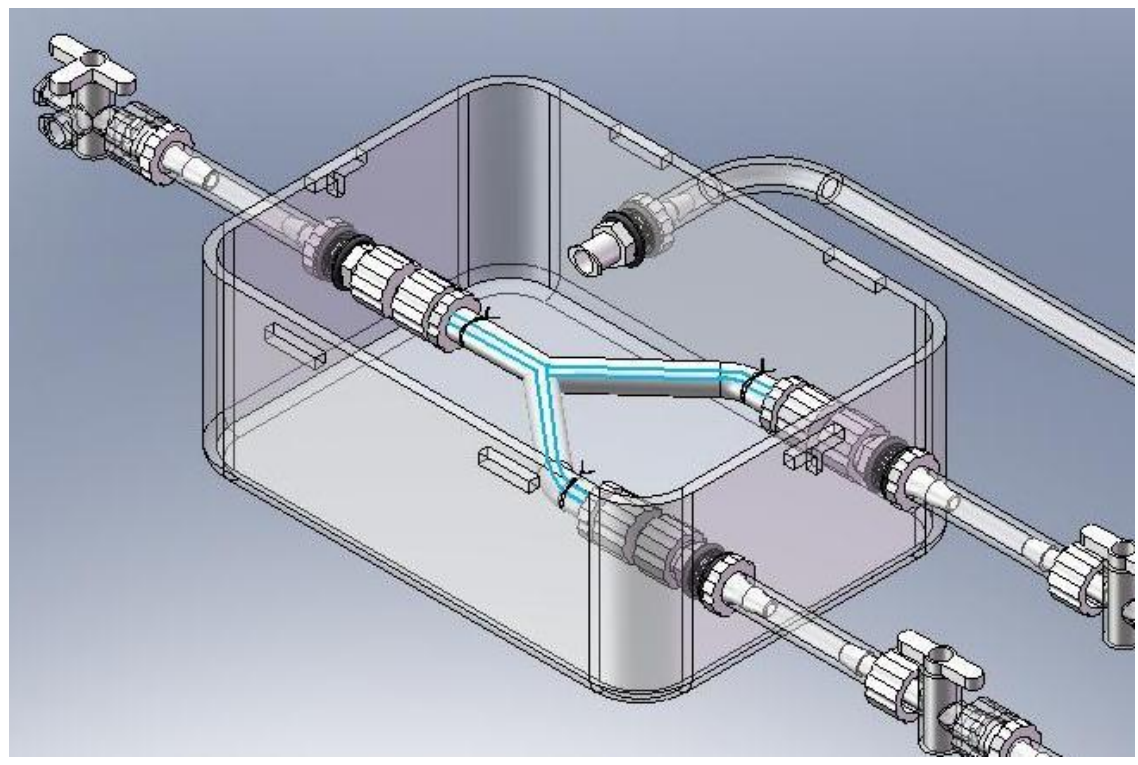

Figure 4.14- Scaffold setup for Y-shaped bifurcation vessels. A Y-shaped bifurcation scaffold can be accommodated in a modified BVM chamber by moving the extra luminal outlet to the long side of the chamber and adding a second luminal outlet in its place.

The second bifurcation geometry that can be created requires one simple modification to the configuration of the Ideal BVM system. By adding a second luminal outlet to the long side of the chamber a T-shaped bifurcation scaffold can be accommodated. A T-shaped bifurcation scaffold can be setup and connected as described in the previous chapter with the addition of one female luer thread style to 200 series barb, 1/8" ID tubing (Value Plastics P/N FTLL230-1). One male-to-male luer lock connector, with one rotating collar (Qosina P/N 17644) also needs to be attached to the second outlet to hold the scaffold. The easiest way to configure the tubing is to simply add a second Y-connector with 200 series barbs, 1/8" ID tubing (Value Plastics P/N Y230-1) prior to the attachment of the one-way stopcock. In addition, a second one-way stopcock (Value Plastics P/N VP455980) must be added to the second luminal outlet to 
control flow. This modified orientation represents T-shaped bifurcation geometry and can accommodate a T-shaped scaffold. The BVM system for T-shaped bifurcation vessels is modeled below in Figures 4.15 and 4.16.

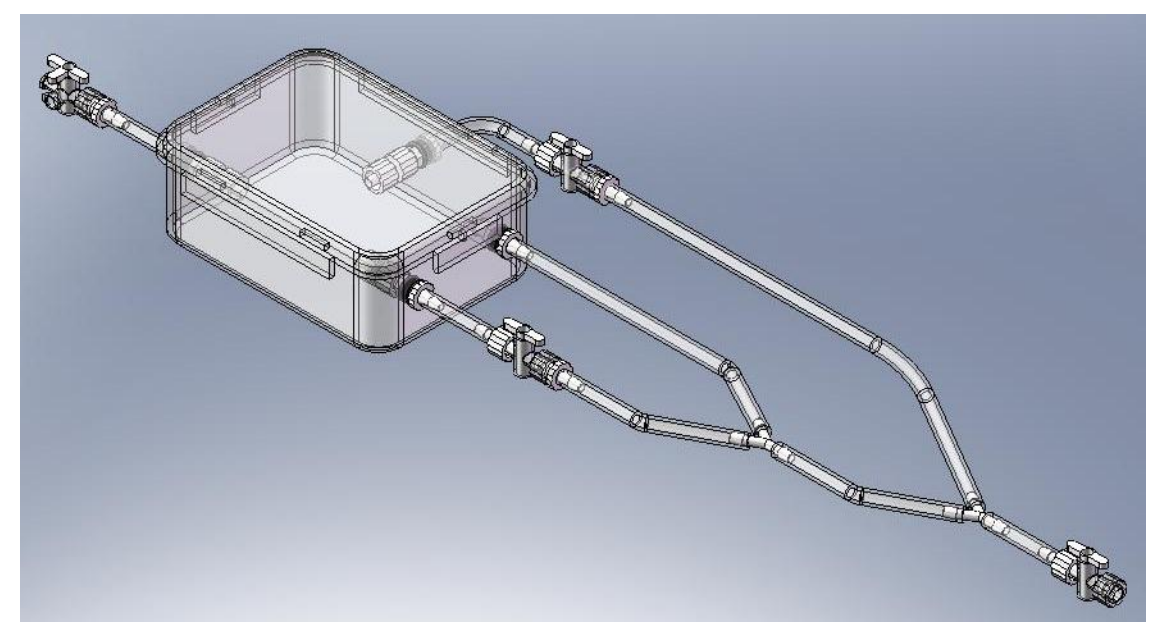

Figure 4.15- BVM system for T-shaped bifurcation vessels.

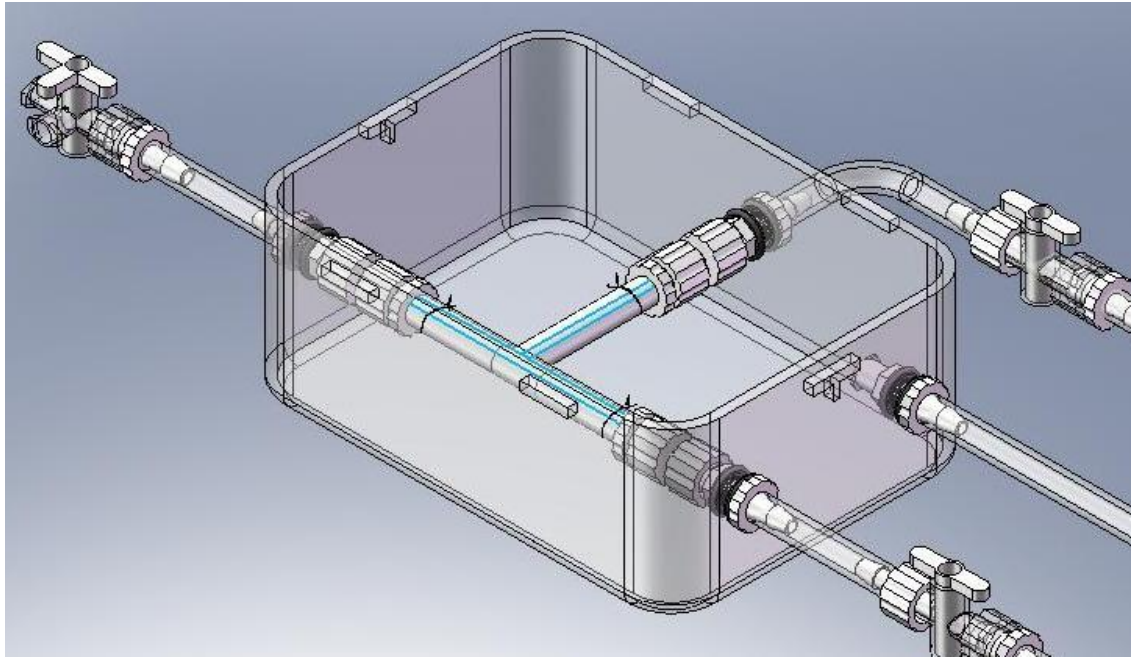

Figure 4.16- Scaffold setup for T-shaped bifurcation vessels. A T-shaped bifurcation scaffold can be accommodated in a modified BVM chamber by adding a second luminal outlet to the long side of the chamber. 
Scaffold setup and insertion for the BVM systems for bifurcation vessels will remain unchanged from the assembly process used for the Ideal BVM system described in the previous chapter. However, Y-shaped and T-shaped bifurcation ePTFE scaffolds will need to be constructed. Sewing standard ePTFE material together in the desired shape could provide a feasible method and is illustrated in Figures 4.17 and 4.18 below.
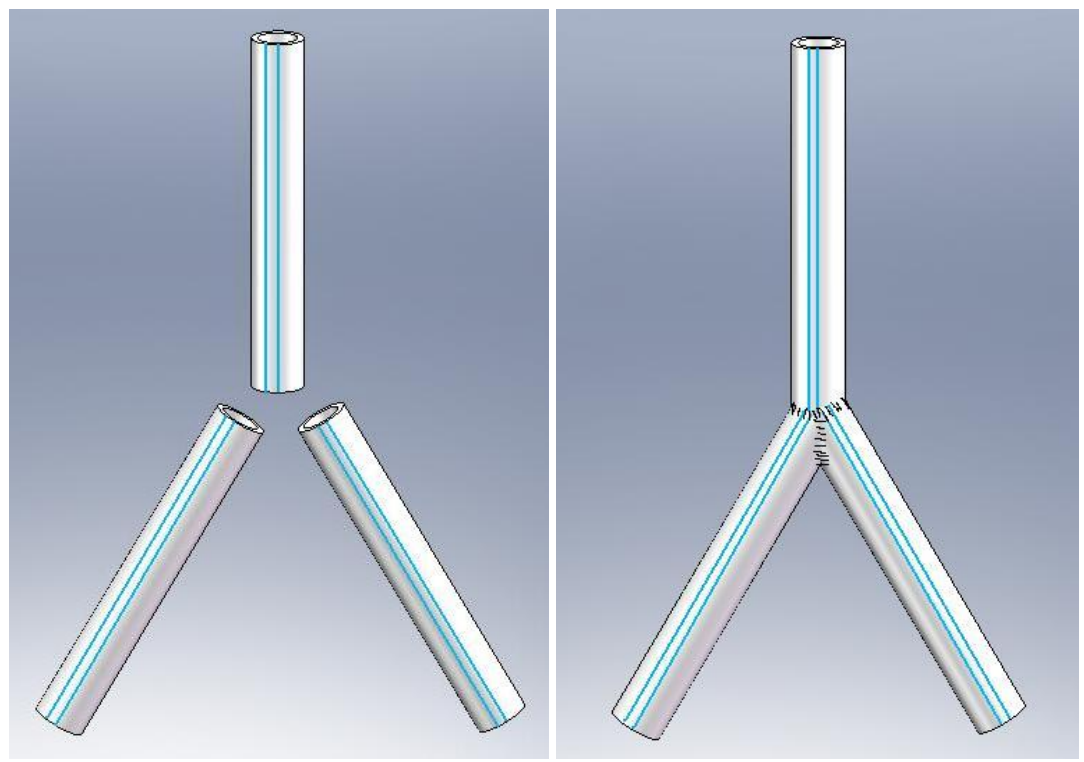

Figure 4.17- Y-shaped bifurcation scaffold. Constructed by sewing three short pieces of ePTFE material together. 


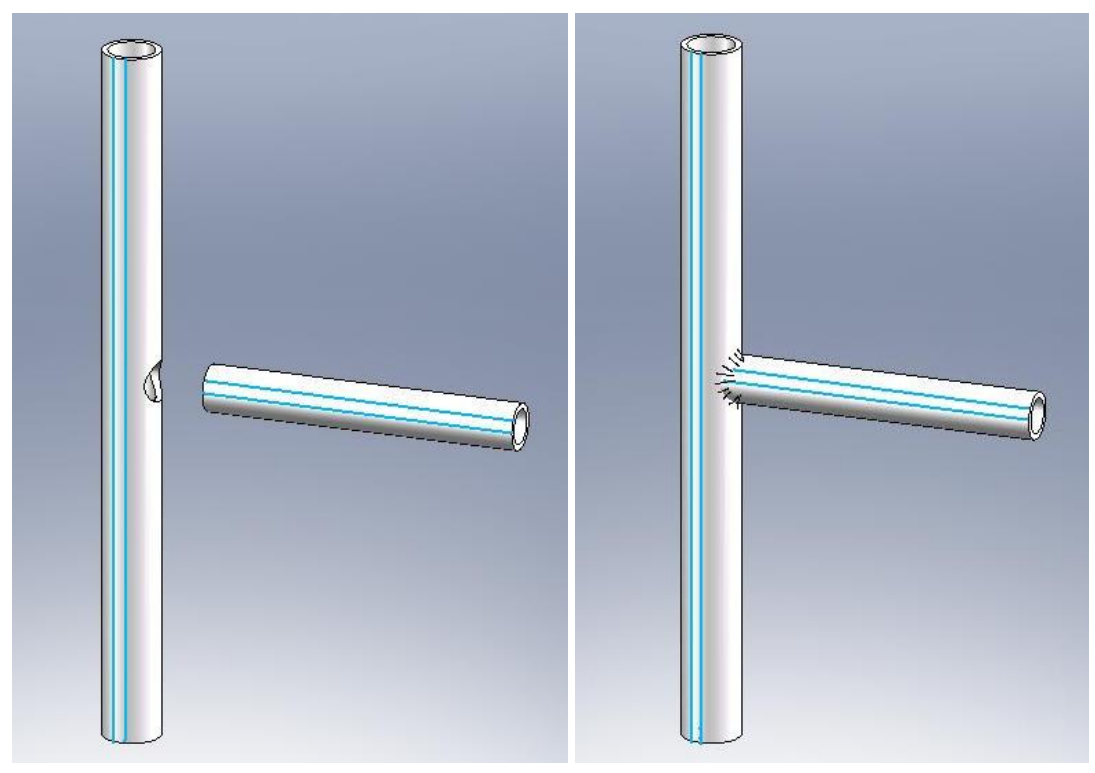

Figure 4.18- T-shaped bifurcation scaffold. Constructed by creating a hole in the center of a long piece of ePTFE material and sewing a short piece of ePTFE material to the hole.

One challenge that may be encountered with this assembly process is leakage at the suture sites. Prototype scaffolds will need to be constructed and inserted into the BVM chambers and flow via a peristaltic pump will need to be initiated to evaluate the potential leakage. Using a mold or customized model to form the entire scaffold in a bifurcation shape could also provide a solution and would eliminate the possibility of leakage, although a material other than ePTFE would need to be chosen and evaluated. As in the previous design, male luer integral lock ring to female luer thread style couplers can be used to vary the overall scaffold lengths in each design. Prototypes of each model are needed to establish the corresponding scaffold lengths versus number of couplers. 
The designs of the BVM systems for bifurcation vessels are very similar to the Ideal BVM system and use the exact same chamber. As a result the BVM chambers for bifurcation vessels meet all of the design criteria established in the previous chapters. 


\section{Next Generation BVM System Prototypes}

Six modified BVM systems representing complex coronary vessel geometries were modeled above: long, slightly bent, moderately angulated, severely angulated, Yshaped bifurcation and T-shaped bifurcation. Although full implementation and testing of these models is beyond the scope of this thesis, a multipurpose and Y-shaped bifurcation BVM system prototype were built with the intention of verifying the feasibility of these designs. The only configuration that was not prototyped was the BVM system for long vessels, since the configuration is identical to the Ideal BVM system with the exception of a longer chamber.

\section{Multipurpose BVM System}

Instead of building each individual BVM system prototype, a multipurpose BVM system was built with appropriate luminal inlets and luminal outlets to accommodate complex vessel configurations. This modified BVM chamber accommodated slightly bent, moderately angulated, severely angulated, and T-shaped bifurcation configurations. The luminal inlets and luminal outlets that were not required for a specific configuration were simply occluded using a male luer integral lock ring plug (Value Plastics P/N LP41). As proposed, two pieces of ePTFE material were sutured together to make the Tshaped bifurcation scaffold. The multipurpose BVM system prototype, with a slightly bent scaffold, a moderately angulated $90^{\circ}$ scaffold, a severely angulated $180^{\circ}$ scaffold, and a T-shaped bifurcation scaffold are shown below in Figures 4.19 through 4.22. 

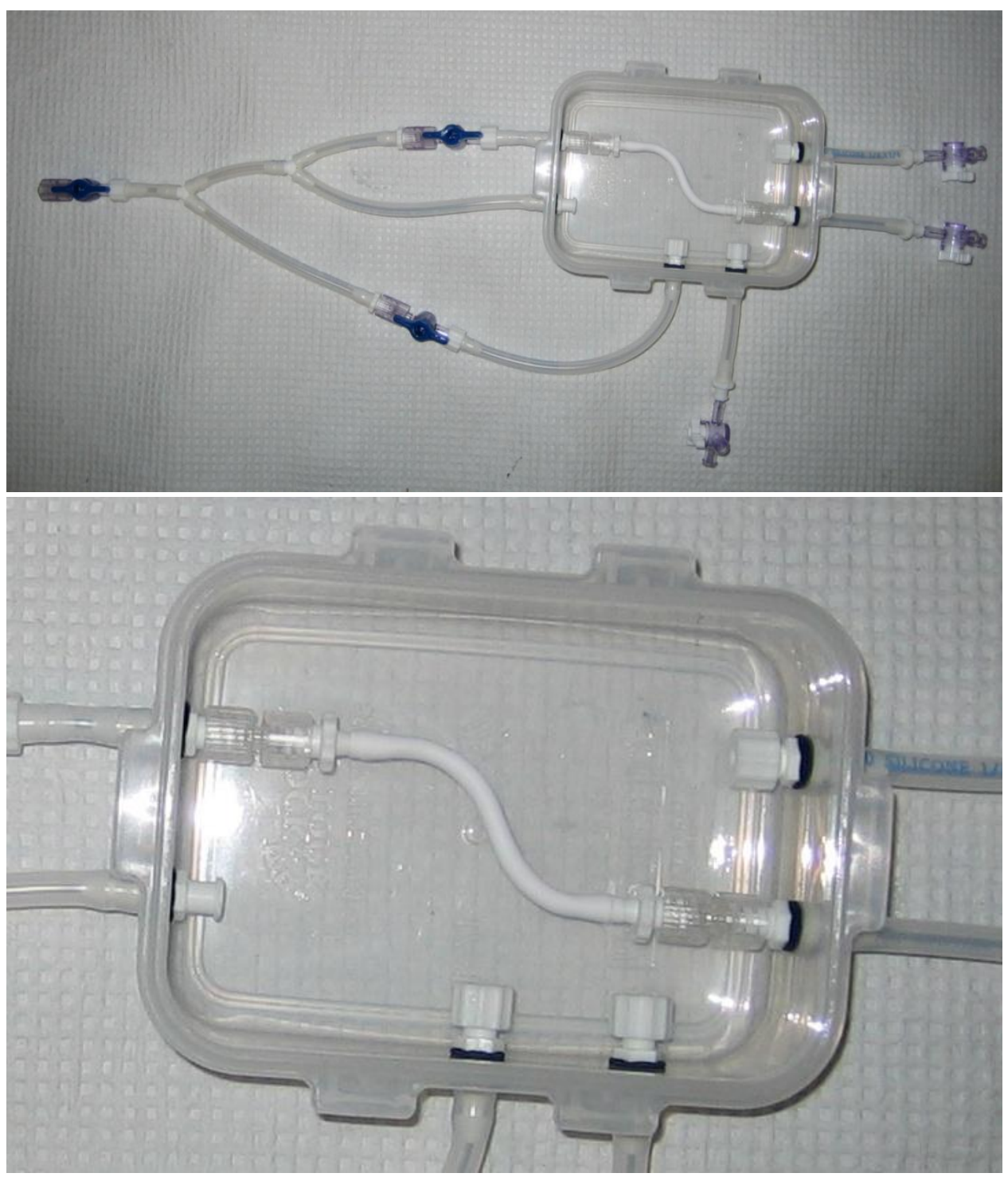

Figure 4.19- Multipurpose BVM System with a slightly bent scaffold. 

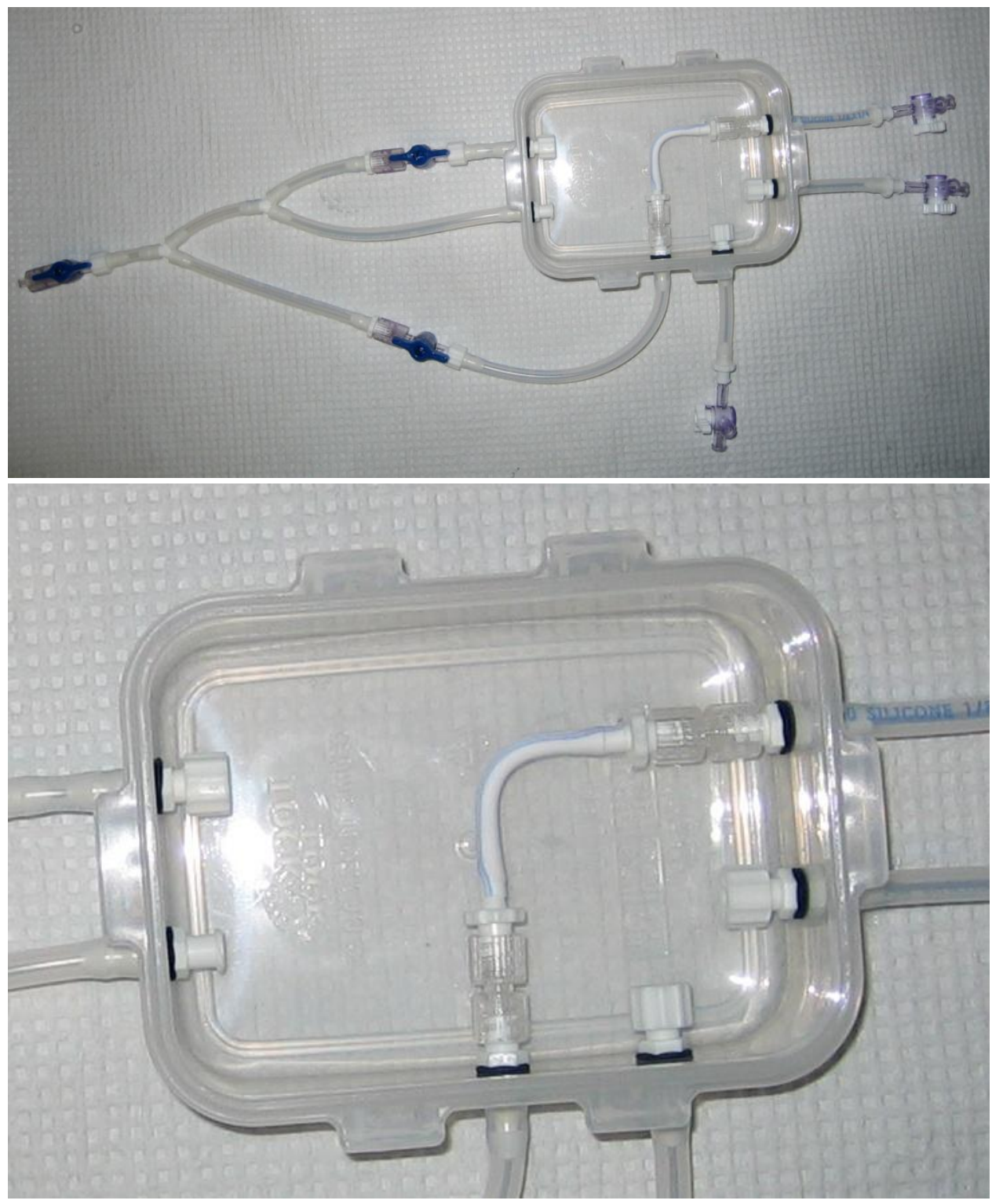

Figure 4.20- Multipurpose BVM System with a moderately angulated $90^{\circ}$ scaffold. 

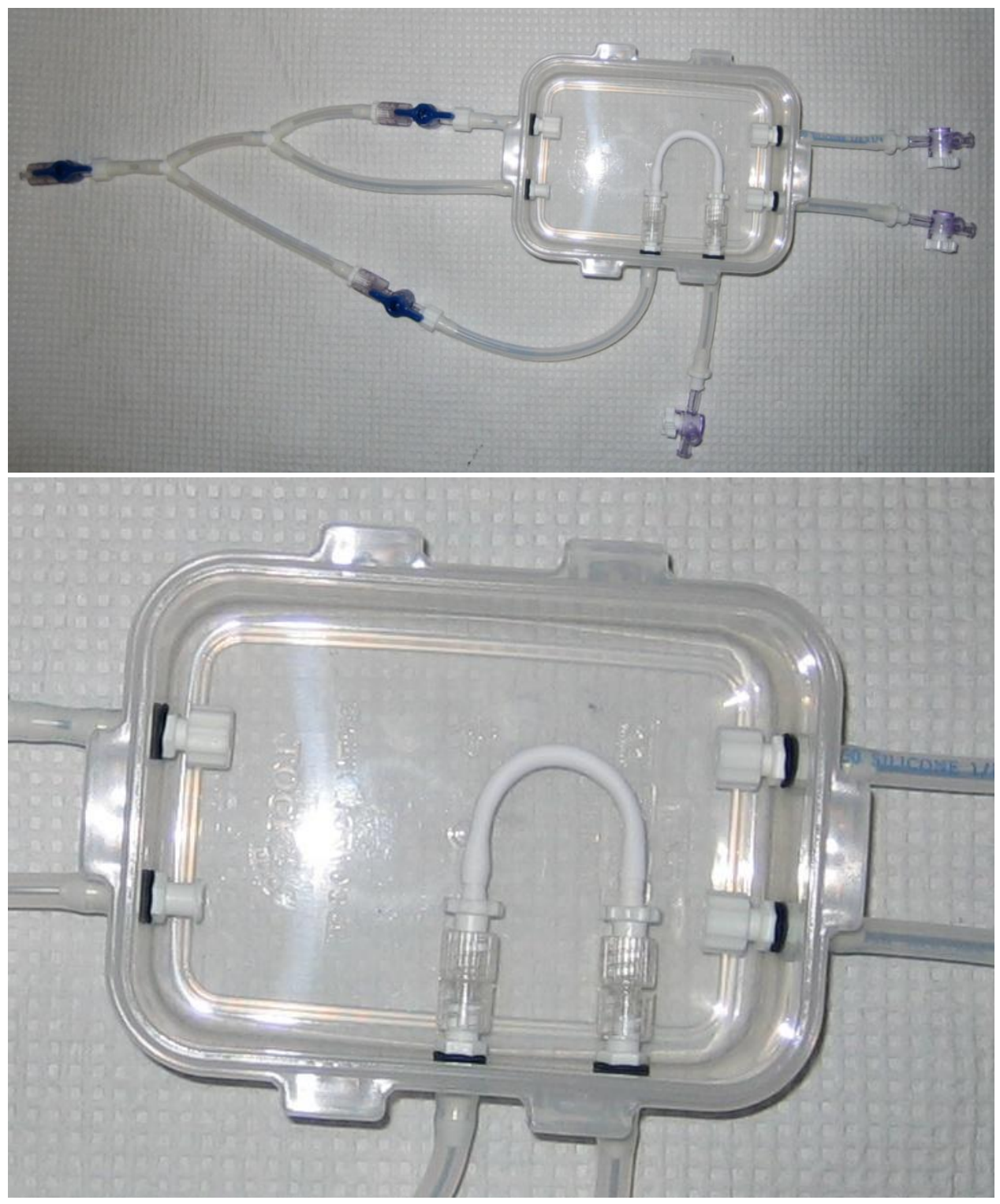

Figure 4.21- Multipurpose BVM System with a severely angulated $180^{\circ}$ scaffold. 

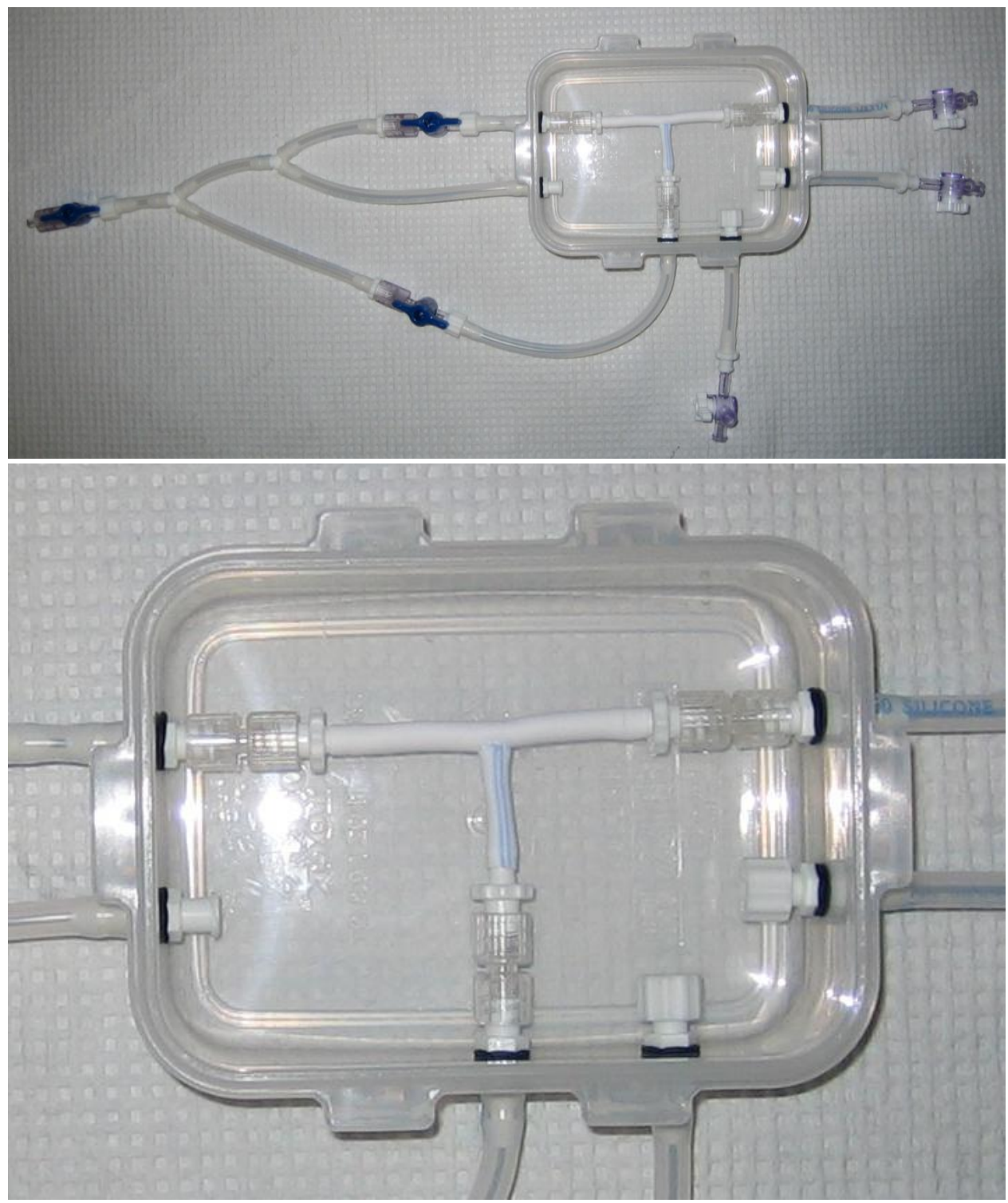

Figure 4.22- Multipurpose BVM System with a T-shaped bifurcation scaffold. 
Y-Shaped Bifurcation BVM System

A Y-shaped bifurcation BVM system was built with appropriate luminal inlets and luminal outlets to accommodate a Y-shaped bifurcation vessel configuration.

However, instead of suturing three separate piece of ePTFE together, as purposed, a simpler method was used. Due to the flexibility of ePTFE material and the desire to use as few sutures as possible, the arms of the T-shaped scaffold were bent upwards to create a Y-shaped scaffold. This method eliminated the need for three separate ePTFE pieces to be sutured together, reducing the chance of leakage at the suture points. The Y-Shaped Bifurcation BVM system prototype is shown below in Figure 4.23. 

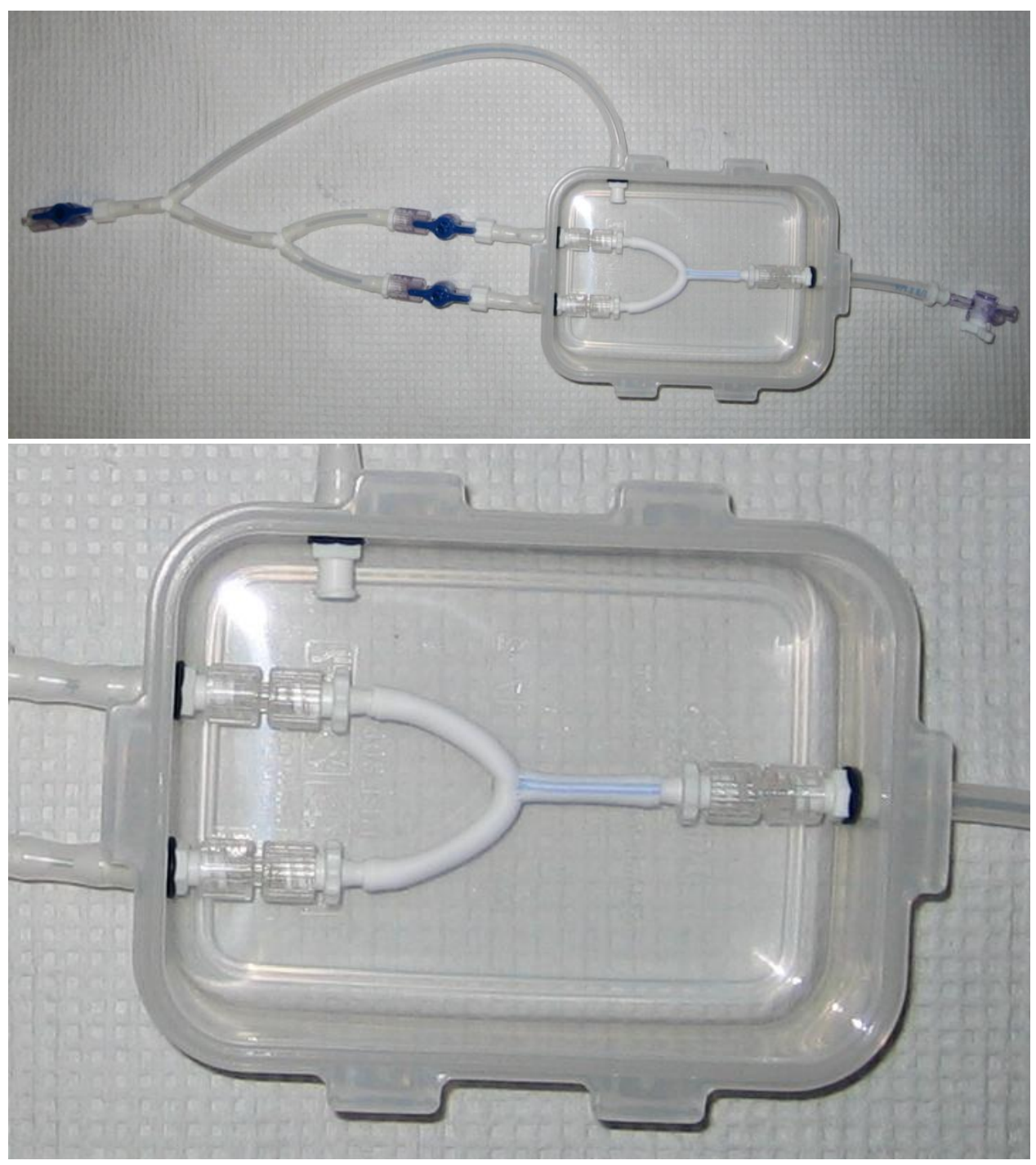

Figure 4.23- Y-Shaped Bifurcation BVM System with a Y-shaped bifurcation scaffold. 


\section{Discussion and Conclusions}

The next generation BVM systems, presented through computer-generated models and initial prototypes, represent the development of new and innovative designs for the evaluation of more complex lesions that currently have not been adequately addressed in preclinical models and support the hypothesis that the Ideal BVM system can be modified to accommodate advancing technology. The work in this chapter expanded the BVM concept to meet the needs of long, angulated and bifurcation lesions. Alternate chambers for the evaluation of more complex vascular devices, as potential future uses of the in vitro blood vessel mimic for preclinical device testing, were illustrated and prototyped.

Full implementation and testing of the modified BVM systems was beyond the scope of this thesis. Additional prototypes and verification testing is needed to demonstrate that the modified BVM systems developed in this chapter meet the testing needs for long, angulated and bifurcation lesions. The prototypes of each modified BVM chamber need to be finalized and the entire bioreactor system needs to be assembled and connected to a peristaltic pump. Scaffolds for each system also need to be assembled, measured and connected to the modified BVM systems to establish lengths, and evaluate the possibility for kinking or occlusion due to bent orientations. Bifurcation scaffolds will need to be constructed and evaluated for their ability to function under pressure and flow conditions.

The first step in the evaluation of each model requires full construction of the modified BVM systems. Construction of the modified BVM chambers utilize the same 
materials and methods described in the previous chapter and detailed in Appendix C.

Location of the luminal inlets and outlets vary between models, but the assembly process remains unchanged. The construction of a multipurpose and Y-shaped bifurcation BVM chamber prototype and the assembly of each complex vessel scaffold configuration within these chambers were shown, verifying the feasibility of these designs.

The complete bioreactor configurations, although not illustrated in this chapter, consist of the modified BVM systems and two-port media reservoirs, described in the previous chapter, connected in series. Setup is identical to the Ideal BVM bioreactor configuration and only two simple connections need to be made. Specific placement of male luer lock and female luer thread fittings ensure proper setup and orientation every time. Only one configuration exists for setting up the modified BVM bioreactor systems, since the fittings of all modified BVM chambers and two-port media reservoirs were designed as male-to-female connections.

The luminal inlet, luminal outlets and extra luminal outlet tubing configurations are identical to the Ideal BVM system and permit variation in flow path. Transluminal and transmural flow are easily controlled via the one-way stopcocks attached to the luminal outlets. For the bifurcation models, one-way stopcocks are attached to both the main vessel luminal outlet and the side branch luminal outlet, allowing control of flow through each of the bifurcation paths. By closing off the luminal outlet paths, through the use of the one-way stopcocks, transmural flow through the porous ePTFE scaffold walls is created, with flow exiting the extra luminal outlets. Transmural flow is used for conditioning and cell sodding of the scaffold material. By opening the one-way 
stopcocks, transluminal flow is restored through the luminal outlets. The one-way stopcocks are easy to use and provide a quick and simple method for controlling the flow paths. The levers are easy to turn and once in position flow is immediately and firmly blocked. The merging of the luminal outlets and extra luminal outlets into one main outlet via Y-connectors make attachment to the two-port media reservoir simple with the need for only one connection. The three-way stopcocks attached to the luminal inlets easily function as both flow paths and cell sodding ports. With two female luer thread fittings, the three-way stopcocks allow the modified BVM systems to be connected to the two-port media reservoirs as well as maintain an extra port for cell sodding and media injection.

Few models, other than animal and clinical studies, exist for preclinical testing of complex intravascular devices designed specifically for placement in complex coronary lesion geometries. Currently most preclinical biocompatibility and tissue response testing is done on animals in outside laboratories which is both time consuming and expensive, especially when testing involves device placement and orientation in long, angulated and bifurcation vessels. Although preclinical animal studies are accepted as a means to predict the probability of device safety and effectiveness in humans, the need exists for preclinical testing using live human tissue. Preclinical testing using human cells would greatly increase the medical community's understanding of tissue response to complex intravascular devices prior to animal and patient testing and prior to device release into the market. Presently, it is difficult to simulate device and tissue interaction and research still needs to be done to fully understand tissue behavior and the in vivo interaction that occurs between device and tissue in complex coronary lesion geometries. 
In order to address some of these limitations, there has been research on the use of alternate or in vitro models for this type of complex device testing. One research group that wanted to evaluate the use of coronary stents in the side branch of a bifurcation lesion constructed a testing model made of Plexiglas with a $4.5 \mathrm{~mm}$ bifurcation shaped trough bored out of the center in order to simulate a bifurcation vessel (Ormiston, et al. 1999). This type of synthetic model approach is not uncommon, and the model does allow stents to be deployed and evaluated in a bifurcated geometry, however no cellular lining exists to provide physiological response evaluations. A second research group that wanted to evaluate the response of endothelial cells to flow hemodynamics at an arterial bifurcation apex constructed a T-shaped bifurcation in vitro flow chamber (Szymanski, et al. 2008). This chamber was custom designed and constructed of polycarbonate, stainless steel, and nylon, which are all autoclavable materials. This model, unlike the previous model, was constructed for the purpose of establishing a cellular lining at the bifurcation apex. However, intravascular devices were not deployed in this model and it is not clear if the design allows for this type of evaluation.

With increasing complexity of coated stents and devices that interface with cells and tissue (Waksman 2007; Waksman 2002; Sharkawi, et al. 2007) the ability to evaluate in a living environment is crucial. The modified BVM systems developed in this chapter were designed to support the growth of 3-dimensional in vitro tissue engineered blood vessel mimics in order to provide physiological models of complex coronary geometries. With advancing technology these modified BVM systems may one day provide an ideal environment for evaluation of complex vascular devices and direct animal and clinical studies toward the most promising advances. 


\section{DISCUSSION AND CONCLUSIONS}

To summarize, the work in this thesis describes the design and optimization of an in vitro blood vessel mimic (BVM) bioreactor system for the evaluation of intravascular devices, specifically coronary stents, in simple and complex vessel geometries. The general hypothesis driving this thesis project was that design changes could be made and new configurations implemented to the existing blood vessel mimic system (Cardinal, et al. 2006) that would improve function and usability for preclinical device testing. In order to test this hypothesis, research was conducted based on two specific aims. The first aim was to develop a more ideal BVM system. The specific hypothesis in this case was that a BVM chamber could be created to accommodate a wider range of stent lengths and diameters, while allowing for easy graft insertion and seal-ability. The second aim was to develop next generation BVM systems, focused on future needs and technology. The hypothesis in this case was that with emerging technology the ability to test stents specifically for bifurcation lesions, as well as in long and angulated vessels, will be needed and is possible within the BVM system. The prototypes developed and the findings from constructed models were presented and discussed in the previous chapters and support the hypotheses stated above.

The more ideal BVM bioreactor system designed and developed in this thesis was successfully built and implemented. A more user friendly design was one of the primary needs met by the Ideal BVM system. Easy scaffold insertion, without the need for bending or twisting, along with a method to quickly and simply seal the vessel chamber without the need for tools, greatly improved the usability of the BVM system. As 
discussed in detail in the introduction to this thesis, stents range in shape and size, and the Ideal BVM system was designed to accommodate stent sizes ranging from 2.0-5.0 mm diameters and 8-38 $\mathrm{mm}$ lengths in order to fit the variations of coronary arteries observed in clinical practice and the stents currently available in the market. In addition, the Ideal BVM chamber was easy to manufacture without the need for adhesive or special tooling and was constructed of materials and components that were readily available and easy to obtain resulting in a low cost design that was easy to scale up. Multiple chambers could be configured in the incubator and the use of biocompatible materials allowed cells to grow and function. Therefore, the work described in this thesis supports the use of the Ideal BVM system for cultivation of in vitro blood vessel mimics.

The next generation BVM systems, presented in this thesis through computergenerated models and initial prototypes, represent the development of new and innovative designs for the evaluation of more complex lesions that currently have not been adequately addressed in preclinical models. The work presented supports the hypothesis that the Ideal BVM system can be modified to accommodate advancing technology. Alternate chambers for the evaluation of more complex vascular devices, as potential future uses of the blood vessel mimic system for preclinical device testing, were illustrated and prototyped. Full implementation and testing of the modified BVM systems was beyond the scope of this thesis. However, the construction of a multipurpose and Yshaped bifurcation BVM chamber prototype and the assembly of each complex vessel scaffold configuration within these chambers were shown, verifying the feasibility of these designs. With advancing technology, these modified BVM systems may one day 
provide an ideal environment for evaluation of complex vascular devices in order to direct animal and clinical studies toward the most promising technologies.

The goal of this thesis was to not only expand upon the original BVM model, but to develop new and innovative models for the evaluation of devices in more complex geometries such as bifurcations, long and angulated vessels. The BVM systems developed in this thesis are model systems which were developed for the initial testing of newly emerging intravascular technology, and represent the next step in preclinical device testing. In the following sections, key aspects of this field and relevant areas of research will be discussed with regard to the present thesis work. 


\section{Preclinical Intravascular Device Testing}

Preclinical device testing is a crucial step in the process of developing and releasing new medical devices to the market. The objective of preclinical testing is to provide preliminary evidence of product safety and efficacy in order to support the initiation of human clinical trials. Preclinical testing typically begins using models and methods that are simple and cost-effective in order to identify potential failures as early in the development process as possible, and then progresses to models with increasing complexity. As discussed in the introduction, preclinical intravascular device testing is currently performed using both in vitro laboratory models and in vivo animal models and provides critical information about cellular and tissue responses as well as device success.

Although preclinical animal studies are accepted as a means to predict the probability of device safety and effectiveness in humans, no animal model can provide complete insights into the human healing response following stent implantation. Preclinical testing using human cells would greatly increase the medical community's understanding of tissue response to a specific device prior to animal and patient testing and prior to device release. The Ideal BVM system developed in this thesis represents an intermediate testing environment for assessing human cellular responses to intravascular stents in a manner that combines the advantages of both in vitro and in vivo approaches. The Ideal BVM system mimics the geometries of the coronary arteries and utilizes human cells, which provide the potential for more predictive preclinical results than animal models alone can provide. The Ideal BVM system developed in this thesis 
provides a feasible method to rapidly evaluate human tissue response to varying stent configurations, materials, drugs and coatings as they become available. Although the Ideal BVM system is not a substitute or replacement for animal models, it has the potential to provide researchers with a more relevant in vitro tissue model that can be used for the preclinical evaluation of new intravascular devices. The major advantage of the in vitro blood vessel mimic system developed in this thesis is its potential for consistent high-throughput evaluation of cellular response to advancing intravascular technologies (Cheng, Lai and Kisaalita 2008; Yliperttula, et al. 2008).

\section{Laminar Flow and Wall Shear Stress}

One area that has not yet been addressed as a potential use for the blood vessel mimic (BVM) bioreactor systems developed in this thesis is the study of laminar flow and wall shear stress in stented vessels. Implanted stents can alter coronary flow and induce significant changes in flow hemodynamics, especially in bifurcated and angulated geometries where flow is already disturbed and shear stress is low (Zhu, et al. 2008;

Wahle, et al. 2006; Benard, et al. 2003). These changes result in local modification of the mechanical stress exerted on the endothelial cells lining the vessel wall and several studies have demonstrated that endothelial cell proliferation increases significantly when subjected to weak shear stress and non-laminar flow (Ridger, et al. 2008; Scott 2006).

A number of experiments have been performed to study the influence of an implanted stent on flow hemodynamics through a stented vessel (Peacock, et al. 1995; Benard, et al. 2003). However, most of these studies used computer generated 
computational techniques rather than animal or in vitro physical models (Balossino, et al. 2008; Dehlaghi, Shadpoor and Najarian 2008). As a result, the influence of flow disturbances on the healing response to an implanted stent is poorly understood and the need exists for anatomically realistic testing models with cellular linings to obtain more predictive preclinical results.

The use of the BVM systems developed in this thesis to study flow hemodynamics in stented vessels could provide important information about the influence of flow and shear stress on vascular healing. The BVM system could be used to not only study the cellular response to laminar flow and shear stress changes due to the presence of a stent, but could provide a model that would simultaneously allow for direct flow and velocity measurements. The BVM bioreactor system created in this thesis is a closed system in which all physical parameters, including flow, can be tightly controlled and monitored while allowing visualization and access to the inner lumen of the stented vessel. These advantages make the in vitro BVM system an ideal model for the study of flow hemodynamics.

\section{Drug Elution Kinetics}

Blood flow through a stented vessel not only affects laminar flow and wall shear stress, but plays a direct role on drug elution kinetics, or drug release, from drug eluting stents (Umashankar, Hari and Sreenivasan 2007). As discussed in the introduction to this thesis, a drug eluting stent is a device that releases a single or multiple bioactive agents into the bloodstream that affect the tissue adjacent to the stent. Currently there are three 
different approaches to binding drugs to coronary stents: drug binding by means of a polymer on the stent surface, drug binding aided by an inorganic stent coating, or direct drug binding to the stent surface without coating (Acharya and Park 2006). Most often drugs are either chemically bonded to the surface of the stent or trapped within a polymer film or porous ceramic that allows controlled drug release (Kandzari, Tcheng and Zidar 2002). Drug release in biodegradable coatings is achieved through disintegration of the biodegradable polymer of the stent, while non-biodegradable coatings release drugs by allowing the drugs to diffuse through a porous matrix. Whatever mechanism of drug binding used flow characteristics can have a significant influence on drug release and distribution within the tissue of the stented vessel (Neubert, et al. 2008).

Currently, most preclinical drug elution studies consist of immersion of the drug eluting stent in a small volume of liquid, with drug concentration measurements taken over time or the use of computer generated computational models (Neubert, et al. 2008; Balakrishnan, et al. 2007; Alexis, et al. 2004). These methods allow for the examination of drug release but lack simulation of the vessel wall and cellular response and their possible influence on drug release and distribution. The examination of in vivo release and distribution is also limited since the drug concentrations in animals and humans cannot reliably be estimated due to low systemic blood concentrations and the fact that blood levels do not necessarily correspond to drug concentration at the stented lesion (Neubert, et al. 2008). Due to these limitations an in vitro model would be extremely valuable for the preclinical evaluation of drug elution kinetics. 
The use of the in vitro BVM system developed in this thesis could provide a beneficial model for the study of drug elution kinetics. The BVM system provides an environment that could allow for observation and measurement of drug release and distribution within the vessel. With the addition of a cellular layer, the cellular response to drug concentrations in the blood and tissue could be better understood and a closed bioreactor system would permit precise drug concentration measurements at specific points in time. Drug concentrations in the BVM system could be easily measured in the circulating media, as well as in the media surrounding the scaffold, and in the scaffold and cellular layer itself. These types of measurements could provide important information on the influence of living tissue to drug release, distribution and local tissue affects which in turn could provide insight into the role of drug eluting stents on the cellular healing response and restenosis.

\section{Delivery Systems}

Much of this thesis has focused on the use of the BVM system to evaluate tissue response to implanted stents. However, as discussed in the introduction, in addition to the advancements in coronary stent design, the method of delivering these therapies has also improved. These improvements have made percutaneous coronary intervention procedures easier to perform and have reduced the amount of time for a single procedure.

Percutaneous coronary intervention in complex coronary geometries is difficult and the increased complexity results in reduced success rates (Saeed, Banerjee and Brilakis 2008). Therefore, novel delivery systems, smaller catheters, lower profile 
balloons and other devices have been created to overcome the challenges associated with tortuous anatomies. Stent delivery is an area of active research and a living model system for testing and evaluating these new delivery devices would be ideal for development and progression of the field.

The future of the BVM system developed in this thesis has the potential to be used not only to evaluated tissue response to stent implantation but to the stent delivery processes and systems themselves. The BVM system could allow researchers to quickly evaluate the ability of the delivery system to safely and effectively implant a stent into a coronary vessel, with the advantage of observing tissue response to the delivery device, as well as the implanted stent. 
The BVM Concept for Other Tissues

Given the possible variability of the general BVM system concept, the following section will explore the potential to create chambers for the evaluation of medical devices in varying physiological tissue models. The BVM system developed in this thesis was designed to mimic coronary blood vessels and vascular tissue. However, the general concept can be used to model any tube like structure in which flow is present and has the potential to provide in vitro models of a wide variety of human tissues.

\section{Urology}

The ureter is a narrow muscular tube in which urine flows from the kidney to the bladder. A ureter may become obstructed as a result of a number of conditions including strictures, inflammation, cancer and kidney stones. When the ureter is obstructed, urine is unable to drain from the kidney and leads to pain, infection and if left untreated, kidney failure. Therefore, action must be taken to restore the flow of urine to the bladder.

Ureteral stenting represents a minimally invasive procedure in which a ureteral stent, a small diameter plastic tube with pigtail shaped ends, is temporarily placed between the kidney and bladder to restore the flow of urine and provide a physical scaffold for the healing ureter. Ureteral stents have widespread use in current urological practice and are used for a wide variety of indications, the most common being the relief of urinary stone disease. Ureteral stents are used to maintain ureteral patency after endourological procedures and relive ureteral obstruction. 
One significant complication associated with ureteral stent placement is encrustation within the stent lumen, blocking the flow of urine (Laube, et al. 2008; Tunney, Keane and Gorman 1997). Infection and pain are also common complications. As a result a number of new ureteral stent designs, materials, and coatings have been introduced to the market with the goal of preventing bacterial buildup and encrustation while increasing patient comfort (Kleinen, Böde and Laube 2008; Wignall, et al. 2008; Cadieux, et al. 2006; Hafron, et al. 2006).

Several in vitro models have been developed for the preclinical testing of ureteral stents (Gorman, et al. 2003; Choong, Wood and Whitfield 2000; Stoller, et al. 2000). These models allow ureteral stents to be deployed and evaluated in anatomical geometries; however, no cellular lining exists to provide physiological response evaluations. Through the creation of an in vitro tissue engineered ureter construct a model system could be established for the initial testing of newly emerging urological technology. The need exists for new and advanced preclinical urological testing models that provide medical device manufacturers and researchers with the ability to quickly evaluate tissue response to new ureteral stent designs, materials and coatings.

\section{Abdominal Aortic Aneurysm}

Abdominal aortic aneurysm (AAA) is a common vascular disorder affecting approximately $5 \%$ of the male population over 55 years of age (Chong and How 2004). An abdominal aortic aneurysm occurs when a weak area of the abdominal aorta expands or bulges due to the high pressure of blood flowing through it. AAA is a significant 
health risk since the aneurysm can burst or rupture at any time leading to severe bleeding, shock and death (Iribarren, et al. 2007). Therefore, action must be taken to ensure that if rupture or leakage occurs, blood flow through the abdominal aorta is not disrupted.

Until recently, open surgical replacement of the diseased aortic segment with a prosthetic graft was the standard treatment for AAA (Steingruber, et al. 2006; Chong and How 2004). Endovascular aortic aneurysm repair (EVAR), however, has become an increasingly successful minimally invasive procedure for treating AAA. During EVAR an endovascular stent-graft is permanently placed within the abdominal aorta at the site of the aneurysm. The stent-graft provides a path for blood to flow through the aneurysm, reducing the pressure exerted on the weakened walls.

There are significant complications associated with EVAR due to the high velocity flow and pressures present in the abdominal aorta. Complications include downstream migration of the stent-graft and leakage at the attachment site (Steingruber, et al. 2006). As a result development of the ideal endovascular stent-graft is ongoing and the need exists for an in vitro AAA flow model for evaluating endovascular fixation techniques, new stent-graft designs and materials, long term durability and the cellular response to these new technologies (Sulaiman, et al. 2008). Through the creation of an in vitro tissue engineered large diameter vessel construct a model system could be established for the initial testing of newly emerging AAA technology. 
Potential as a Commercial Device

The BVM systems developed in this thesis have the potential to be created on a large scale and sold to the biomedical industry as commercial preclinical testing devices. The BVM system could be marketed as an in vitro bench-top testing apparatus that mimics the human coronary artery environment. The BVM system could allow medical device manufacturers and researchers to conduct their own individual preclinical biocompatibility testing on a number of designs using a predictive model which could reduce the number of animal studies required. The Multipurpose BVM, especially, has the potential to be an ideal preclinical model since simple and complex vessel scaffolds can be accommodated allowing researchers to test devices in a number of vessel geometries. This type of preclinical testing may lead to better understanding of device and tissue interaction much earlier in the development process and give researchers the chance to make design improvements prior to animal and clinical studies.

In order to market the BVM system as a commercial device, additional work would need to be completed. Extensive studies and comparisons would need to be made between the results obtained from the BVM system and animal models. Research would be needed to demonstrate that the BVM system provides predictive preclinical results that are comparable to those obtained from animal and clinical studies. Besides additional laboratory testing, ship testing would need to be performed to ensure that the BVM system can be transported without sustaining physical damage. In addition, testing would need to be conducted to ensure that the cells provided with the system are stored and packaged properly so that they survive shipment. Other considerations that would need to 
be addressed include determining the number of times the system can be sterilized and reused prior to failure, the creation of detailed instructions for use, and large scale manufacturing ability. 


\section{Limitations and Future Work}

The in vitro tissue engineered blood vessel mimic (BVM) systems developed in this thesis and their feasibility for the initial testing of newly emerging intravascular technologies has been demonstrated. There are limitations that exist with these designs, however, and the focus of this section is to discuss the limitations of the current models and future improvements that can be implemented to improve their design.

One design criteria that was not taken into consideration during the Ideal BVM development was the accommodation of physiological pressure and flow. The current BVM system setup uses low flow rates and low pressures. The performance of the Ideal BVM system has not been tested under high pressure, however, the thin compliant chamber walls may lead to failure. Under high pressure the walls may bellow, due to their flexibility, creating back pressure within the system. The ability of the lid to remain tightly sealed may also be compromised. Therefore, to improve the Ideal BVM system design it may be advantageous to use a container with more rigid walls that do not deform under high pressure. A stronger latching system may also be needed to prevent leakage from the lid.

Although stent delivery and deployment were not performed in the Ideal BVM system designed in this thesis, the procedure remains identical to the original BVM system. Stent delivery and deployment is performed by inserting a balloon catheter mounted with a coronary stent into the bioreactor system through the luminal inlet. Flow is temporarily stopped during the deployment procedure and requires the luminal inlet to be disconnected from the bioreactor system. It would be advantageous if stent delivery 
and deployment could be performed without disconnecting the BVM system and stopping flow. One way that this could be accomplished is by attaching a touhy borst adapter via a Y-connector to a section of tubing leading to the luminal inlet. A touhy borst adapter allows small diameter devices, such as balloon catheters, to be inserted through a small seal which prevents leakage at the insertion site. The addition of this feature could allow stent delivery and deployment to be performed without disrupting flow within the BVM system and represents a more realistic stenting procedure. 


\section{Conclusion}

As discussed in detail throughout this thesis, the blood vessel mimic system has the potential to help researchers predict the response of human tissue to intravascular devices prior to animal and clinical testing. The BVM system represents a more realistic and predictive model that could provide a high-throughput system for assessment of cellular response to novel intravascular technologies in order to direct animal and clinical studies toward the most promising devices. The benefits to medical device manufacturers and more importantly to patients needing these devices would be immense, resulting in better products reaching patients in a more timely fashion and at lower costs. With intravascular technologies advancing at a rapid pace, the BVM system has the potential to one day redefine how intravascular devices, specifically coronary stents, are tested and developed. 


\section{REFERENCES}

Abizaid, Alexandre C., Jose de Ribamar Costa, Robert Whitbourn, and Jean C. Chang. "The CardioMind coronary stent delivery system: stent delivery on a 0.014 " guidewire platform." EuroIntervention 3 (2007): 154-157.

Abrams, Herbert L., Stanley Baum, and Michael J. Pentecost. Abrams'Angiography: Interventional Radiology. Philadelphia: Lippincott Williams \& Wilkins, 2006.

Acharya, Ghanashyam, and Kinam Park. "Mechanisms of controlled drug release from drug-eluting stents." Advanced Drug Delivery Reviews 58, no. 3 (2006): 387-401.

Alexis, Frank, Subbu S. Venkatraman, Santosh Kumar Rath, and Freddy Boey. "In vitro study of release mechanisms of paclitaxel and rapamycin from drug-incorporated biodegradable stent matrices." Journal of Controlled Release 98, no. 1 (2004): 67-74.

American Heart Association. "Heart Disease and Stroke Statistics - 2008 Update." American Heart Association, 2008.

Balakrishnan, Brinda, John F. Dooley, Gregory Kopia, and Elazer R. Edelman. "Intravascular drug release kinetics dictate arterial drug deposition, retention, and distribution." Journal of Controlled Release 123, no. 2 (2007): 100-108.

Balossino, Rossella, Francesca Gervaso, Francesco Migliavacca, and Gabriele Dubini. "Effects of different stent designs on local hemodynamics in stented arteries." Journal of Biomechanics 41, no. 5 (2008): 1053-1061.

Benard, Nicolas, Damien Coisne, Erwan Donal, and Robert Perrault. "Experimental study of laminar blood flow through an artery treated by a stent implantation: characterisation of intra-stent wall shear stress." Journal of Biomechanics 36, no. 7 (2003): 991-998.

Bokhari, Maria, Ross J. Carnachan, Neil R. Cameron, and Przyborski. "Novel cell culture device enabling three-dimensional cell growth and improved cell function." Biochemical and Biophysical Research Communications 354 (2007): 1095-1100. 
Bonnett, Raymond, I. Patrick Kay, Manel Sabate, and Marco A. Costa. Cardiac catheterization and percutaneous interventions. London: Taylor \& Francis, 2004.

Budoff, Matthew J., Ambarish Gopal, Khawar M. Gul, Song S. Mao, Hans Fischer, and Ronald J. Oudiz. "Prevalence of obstructive coronary artery disease in an outpatient cardiac CT angiography environment." International Journal of Cardiology 129 (2008): 32-36.

Cadieux, Peter A., et al. "Triclosan Loaded Ureteral Stents Decrease Proteus Mirabilis 296 Infection in a Rabbit Urinary Tract Infection Model." The Journal of Urology 175, no. 6 (2006): 2331-2335.

Cardinal, Kristen O'Halloran, Garret T. Bonnema, Heidi Hofer, Jennifer Kehlet Barton, and Stuart K. Williams. "Tissue-Engineered Vascular Grafts as In Vitro Blood Vessel Mimics for the Evaluation of Endothelialization of Intravascular Devices." Tissue Engineering 12, no. 12 (December 2006): 3431-3438.

Cardinal, Marie-Hélène Roy, Jean Meunier, Gilles Soulez, Roch L. Maurice, Éric Therasse, and Guy Cloutier. "Intravascular Ultrasound Image Segmentation: A ThreeDimensional Fast-Marching Method Based on Gray Level Distributions." IEEE Transactions on Medical Imaging 25, no. 5 (May 2006): 590-601.

Cenni, E., et al. "Established Cell Lines and Primary Cultures in Testing Medical Devices In Vitro." Toxicology In Vitro 13 (1999): 801-810.

Chen, Huang-Chi, and Yu-Chen Hu. "Bioreactors for tissue engineering." Biotechnology Letters 28, no. 18 (September 2006): 1415-1423.

Cheng, Ke, Yinzhi Lai, and William S. Kisaalita. "Three-dimensional polymer scaffolds for high throughput cell-based assay systems." Biomaterials 29 (2008): 2802-2812.

Cheng, Ke, Yinzhi Lai, and William S. Kisaalita. "Three-dimensional polymer scaffolds for high throughput cell-based assay systems." Biomaterials 29, no. 18 (2008): 28022812. 
Chong, C. K., and T. V. How. "Flow patterns in an endovascular stent-graft for abdominal aortic aneurysm repair." Journal of Biomechanics 37 (2004): 89-97.

Choong, S. K. S., S. Wood, and H. N. Whitfield. "A model to quantify encrustation on ureteric stents, urethral catheters and polymers intended for urological use." BJU International 86 (2000): 414-421.

Colombo, Antonio, Goran Stankovic, and Jeffrey W. Moses. "Selection of coronary stents." Journal of the American College of Cardiology 40, no. 6 (September 2002): 1021-1033.

Connolley, Thomas, Derek Nash, Jean-Yves Buffiere, Faisal Sharif, and Peter Edward McHugh. "X-ray micro-tomography of a coronary stent deployed in a model artery." Medical Engineering \& Physics 29 (2007): 1132-1141.

Crawford, Michael H. Current Diagnosis \& Treatment in Cardiology: Diagnosis \& Treatment in Cardiology. McGraw-Hill Professional, 2002.

Cui, Z. F., X. Xu, N. Trainor, J. T. Triffitt, J. P. G. Urban, and U. K. Tirlapur. "Application of multiple parallel perfused microbioreactors and three-dimensional stem cell culture for toxicity testing." Toxicology In Vitro 21 (2007): 1318-1324.

Deconinck, E., J. Sohier, I. De Scheerder, and G. Van der Mooter. "Pharmaceutical Aspects of Drug Eluting Stents." Journal of Pharmaceutical Sciences, 2008.

Dehlaghi, Vahab, Mohammad Tafazoli Shadpoor, and Siamak Najarian. "Analysis of wall shear stress in stented coronary artery using 3D computational fluid dynamics modeling." Journal of Materials Processing Technology 197 (2008): 174-181.

Desai, Anjali, Ying Zhao, and Jeffrey S. Warren. "Development of atherosclerosis in Balb/c apolipoprotein E-deficient mice." Cardiovascular Pathology 17 (2008): 233-240.

Dotter, Charles T. "Transluminally-placed coilspring endarterial tube grafts. Long-term patency in canine popliteal artery." Investigative Radiology 4 (1969): 329-332. 
Ellis, Stephen G., et al. "Coronary Morphologic and Clinical Determinants of Procedural Outcome With Angioplasty for Multivessel Coronary Disease: Implications for Patient Selection." Circulation (American Heart Association) 82 (1990): 1193-1202.

Engbers-Buijtenhuijs, Paula, et al. "Biological characterization of vascular grafts cultured in a bioreactor." Biomaterials 27 (2006): 2390-2397.

Erbel, Raimund, Carlo Di Mario, Jozef Bartunek, Johann Bonnier, Bernard de Bruyne, and Franz R Eberli. "Temporary scaffolding of coronary arteries with bioabsorbable magnesium stents: a prospective, non-randomized multicentre trial." The Lancet 369 (June 2007): 1869-1875.

Falk, Erling. "Pathogenesis of Atherosclerosis." Journal of the American College of Cardiology 47, no. 8 (April 2006): C7-C12.

Fattori, Rosella, and Tommaso Piva. "Drug-Eluting Stents in Vascular Intervention." The Lancet 361, no. 9353 (January 2003): 247-249.

Fazio, Sergio, and MacRae F. Linton. "Mouse Models of Hyperlipidemia and Atherosclerosis." Frontiers in Bioscience 6 (2001): 515-525.

Fisher, John P., Antonios G. Mikos, and Joseph D. Bronzino. Tissue Engineering. Boca Raton, Florida: CRC Press, Taylor \& Francis Group, 2007.

Fournier, Juan A., Jose M. Hernandez, Jose A. Ramirez, Mariano Valdes, Armando Bethencourt, and Luis Insa. "Early results of direct coronary stenting in consecutive patients when multivessel, complex, long lesions, and small vessels are included." International Journal of Cardiology 104 (2005): 282-287.

Gorman, S. P., C. P. Garvin, F. Quigley, and D. S. Jones. "Design and validation of a dynamic flow model simulating encrustation of biomaterials in the urinary tract." Journal of Pharmacy and Pharmacology 55, no. 4 (2003): 461-468.

Grube, Eberhard, et al. "Six-month clinical and angiographic results of a dedicated drugeluting stent for the treatment of coronary bifurcation narrowings." The American Journal of Cardiology 99, no. 12 (2007): 1691-1697. 
Gruentzig, Andreas R. "Transluminal dilation of coronary artery stenosis." Lancet 1 (1978): 263.

Hafron, Jason, et al. "Novel dual-lumen ureteral stents provide better ureteral flow than single ureteral stent in ex vivo porcine kidney model of extrinsic ureteral obstruction." Urology 68, no. 4 (2006): 911-915.

Hagemeister, Jens, Frank M. Baer, Robert H. G. Schwinger, and Hans W. Höpp. "Compliance of a cobalt chromium coronary stent alloy - the COVIS." Current Controlled Trials in Cardiovascular Medicine 6, no. 17 (October 2005).

Hamm, Christian W., Jacobus Reimers, Thomas Ischinger, Hans-Jurgen Rupprecht, Jurgen Berger, and Walter Bleifeld. "A Randomized Study of Coronary Angioplasty Compared with Bypass Surgery in Patients with Symptomatic Multivessel Coronary Disease." New England Journal of Medicine 16, no. 331 (October 1994): 1037-1043.

Hara, Hidehiko, Masato Nakamura, Julio C. Palmaz, and Robert S. Schwartz. "Role of stent design and coatings on restenosis and thrombosis." Advanced Drug Delivery Reviews 58 (2006): 377-386.

Hayden, Michael, Michael Pignone, Christopher Phillips, and Cynthia Mulrow. "Aspirin for the primary prevention of cardiovascular events: A summary of the evidence for the U.S. Preventive Services Task Force." Annals of Internal Medicine 136, no. 2 (January 2002): $161-172$.

Hoerstrup, Simon P., Gregor Zund, Ralf Sodian, Andrea M. Schnell, Jurg Grunenfelder, and Marko I. Turina. "Tissue engineering of small caliber vascular grafts." European Journal of Cardio-Thoracic Surgery 20 (2001): 164-169.

Holmes, David R., Michael Savage, J. M. LaBlanche, and Lars Grip. "Results of Prevention of REStenosis with Tranilast and its Outcome (PRESTO) trial." Circulation 106 (2002): 1243-1250. 
Iakovou, Ioannis, Lei Ge, and Antonio Colombo. "Contemporary Stent Treatment of Coronary Bifurcations." Journal of the American College of Cardiology 46, no. 8 (October 2005): 1446-1455.

Ikeno, Fumiaki, Maurice Buchbinder, and Alan C. Yeung. "Novel stent and delivery systems for the treatment of bifurcation lesions: porcine coronary artery model." Cardiovascular Revascularization Medicine 8 (2007): 38-42.

Indolfi, Ciro, Annalisa Mongiardo, Antonio Curcio, and Daniele Torella. "Molecular Mechanisms of In-Stent Restenosis and Approach to Therapy with Eluting Stents." Trends Cardiovascular Medicine 13, no. 4 (2003): 142-148.

Iribarren, Carlos, Jeanne A. Darbinian, Alan S. Go, Bruce H. Fireman, Chong D. Lee, and Douglas P. Grey. "Traditional and Novel Risk Factors for Clinically Diagnosed Abdominal Aortic Aneurysm: The Kaiser Multiphasic Health Checkup Cohort Study." Annals of Epidemiology 17, no. 9 (2007): 669-678.

Ischinger, Thomas, Ronald Solar, and Evelyn Hitzke. "Improved outcome with novel device for low-pressure PTCA in de novo and in-stent lesions." Cardiovascular Radiation Medicine 4 (2003): 2-6.

Jeong, Sung In, et al. "Mechano-active tissue engineering of vascular smooth muscle using pulsatile perfusion bioreactors and elastic PLCL scaffolds." Biomaterials 26 (2005): 1405-1411.

Kandzari, David E., James E. Tcheng, and James P. Zidar. "Coronary artery stents: Evaluating new designs for contemporary percutaneous intervention." Catheterization and Cardiovascular Interventions 56, no. 4 (July 2002): 562-576.

Kang, Ho-Jae, Deug Joong Kim, Sung-Joon Park, Ji-Beom Yoo, and Y. S. Ryu. "Controlled drug release using nanoporous anodic aluminum oxide on stent." Thin Solid Films 515, no. 12 (April 2007): 5184-5187. 
Kang, Woong Chol, et al. "Angiographic and intravascular ultrasound study of the effects of overlapping sirolimus- and paxitaxel-eluting stents: Comparison with same drugeluting overlapping stents." International Journal of Cardiology 123 (2007): 12-17.

Kantor, Birgit, Khalid Ashai, David R. Holmes, and Robert S. Schwartz. "The experimental animal models for assessing treatment of restenosis." Cardiovascular Radiation Medicine 1, no. 1 (1999): 48-54.

Karoussos, I. A., et al. "Inorganic Materials as Drug Delivery Systems in Coronary Artery Stenting." Materialwissenschaft and Werkstofftechnik 33, no. 12 (December 2002): 738-746.

Kern, Morton J., et al. "Physiological Assessment of Coronary Artery Disease in the Cardiac Catheterization Laboratory: A Scientific Statement From the American Heart Association Committee on Diagnostic and Interventional Cardiac Catheterization, Council on Clinical Cardiology." Circulation (American Heart Association) 114 (2006): 1321-1341.

Khan, M. Gabriel. Encyclopedia of Heart Diseases. Elsevier Academic Press, 2005.

King, Spencer B., and Bernhard Meier. "Interventional Treatment of Coronary Heart Disease and Peripheral Vascular Disease." Circulation (American Heart Association) 102 (November 2000): IV81-IV86.

Kini, Annapoorna S. "Coronary Angiography, Lesion Classification and Severity Assessment." Cardiology Clinics 24, no. 2 (May 2006): 153-162.

Kleinen, Lisa, Ulla Böde, and Norbert Laube. "Ex-vivo investigations on the friction behavior of amorphous carbon coated ureteral stents." Diamond and Related Materials 17 (2008): 1746-1750.

Korossis, S. A., F. Bolland, J. N. Kearney, J. Fisher, and E. Ingham. "Bioreactors in Tissue Engineering." Topics in Tissue Engineering 2 (2005): 1-23. 
Kraitzer, Amir, Yoel Kloog, and Meital Zilberman. "Approaches for prevention of restenosis." Journal of Biomedical Materials Research Part B: Applied Biomaterials 85B, no. 2 (May 2008): 583-603.

Lau, Kean-Wah, Koon-Hou Mak, Jui-Sung Hung, and Ulrich Sigwart. "Clinical impact of stent construction and design in percutaneous coronary intervention." American Heat Journal 147, no. 5 (2004): 764-773.

Laube, Norbert, Lisa Kleinen, Vadym Avrutin, Ulla Böde, Andreas Meißner, and Christian Fisang. "The distribution of crystalline material in obstructed stents - In need for intra-luminal surface modification?" Journal of Biomedical Materials Research Part B: Applied Biomaterials 87B, no. 2 (2008): 590-597.

Lefèvre, Thierry, et al. "The FRONTIER Stent Registry: Safety and Feasibility of a Novel Dedicated Stent for the Treatment of Bifurcation Coronary Artery Lesions." Journal of the American College of Cardiology 46, no. 4 (2005): 592-598.

Lewis, A. L., L. A. Tolhurst, and P. W. Stratford. "Analysis of a PhosphorylcholineBased Polymer Coating on a Coronary Stent Pre- and Post Implantation." Biomaterials 23, no. 7 (April 2002): 1697-1706.

Libby, Peter, Paul M. Ridker, and Attilio Maseri. "Inflammation and Atherosclerosis." Circulation 105 (March 2002): 1135-1143.

Louvard, Yves, et al. "Classification of Coronary Artery Bifurcation Lesions: Time for a Consensus." Catheterization and Cardiovascular Interventions 71 (2008): 175-183.

Lowe, Harry C., Stephen N. Oesterle, and Levon M. Khachigian. "Coronary in-stent restenosis: Current Status and Future Strategies." Journal of the American College of Cardiology 39, no. 2 (January 2002): 183-193.

Mani, Gopinath, Marc D. Feldman, Devang Patel, and C. Mauli Agrawal. "Coronary stents: A material perspective." Biomaterials 28 (2007): 1689-1710.

Martin, Ivan, David Wendt, and Michael Heberer. "The role of bioreactors in tissue engineering." Trends in Biotechnology 22 (February 2004): 80-86. 
Miyazawa, Akiyoshi, et al. "Analysis of Bifurcation Lesions Treated with Novel DrugEluting Dedicated Bifurcation Stent System." Catheterization and Cardiovascular Interventions 70 (2007): 952-957.

Morton, Allison, David Crossman, and Julian Gunn. "The influence of physical stent parameters upon restenosis." Pathologie Biologie 52 (2004): 196-205.

Mullany, Charles J. "Coronary Artery Bypass Surgery." Circulation 107, no. 3 (January 2003): 21-22.

Neubert, Anne, et al. "Development of a vessel-simulating flow-through cell method for the in vitro evaluation of release and distribution from drug-eluting stents." Journal of Controlled Release 130, no. 1 (2008): 2-8.

Noll, Georg. "Pathogenesis of atherosclerosis: a possible relation to infection." Atherosclerosis 140 (1998): S3-S9.

Oesterle, Stephen N., et al. "The stent decade: 1987 to 1997." American Heart Journal 136, no. 4 (1998): 578-599.

Ormiston, John A., et al. "A bioabsorbable everolimus-eluting coronary stent system for patients with single de-novo coronary lesions (ABSORB): a prospective open-label trial." The Lancet 371 (March 2008): 899-907.

Ormiston, John A., Mark W. I. Webster, Peter N. Ruygrok, James T. Stewart, Harvey D. White, and Douglas S. Scott. "Stent Deformation Following Simulated Side-Branch Dilatation: A Comparison of Five Stent Designs." Catheterization and Cardiovascular Interventions 47 (1999): 258-264.

Peacock, Jon, Shelly Hankins, Travis Jones, and Robert Lutz. "Flow instabilities induced by coronary artery stents: Assessment with an in vitro pulse duplicator." Journal of Biomechanics 28, no. 1 (1995): 17-26.

Poncet, Philippe. "Trends and Developments in Self-expanding Nitinol Stents." Business Briefing: Medical Device Manufacturing and Technology, 2005. 
Poncin, P., and J. Proft. "Stent Tubing: Understanding the Desired Attributes." Materials \& Processes for Medical Devices. ASM International, 2003.

Popma, Jeffrey J., Bonnie Weiner, Michael J. Cowley, Charles Simonton, Dan McCormick, and Ted Feldman. "FDA Advisory Panel on the Safety and Efficacy of Drug-Eluting Stents: Summary of Findings and Recommendations." Journal of Interventional Cardiology 20, no. 6 (2007): 425-446.

Punchard, M. A., et al. "Endothelial cell response to biomechanical forces under simulated vascular loading conditions." Journal of Biomechanics 40 (2007): 3146-3154.

Ratcliffe, Anthony, and Laura E. Niklason. "Bioreactors and Bioprocessing for Tissue Engineering." Annals of the New York Academy of Sciences 961 (2002): 210-215.

Ridger, Victoria, Rob Krams, Angelo Carpi, and Paul C. Evans. "Hemodynamic parameters regulating vascular inflammation and atherosclerosis: A brief update." Biomedicine \& Pharmacotherapy, 2008: 1-5.

Rieu, Regis, et al. "Assessment of the Trackability, Flexibility, and Conformability of Coronary Stents: A Comparative Analysis." Catheterization and Cardiovascular Interventions 59, no. 4 (2003): 496-503.

Ross, Russell. "Atherosclerosis - An Inflammatory Disease." New England Journal of Medicine 340, no. 2 (January 1999): 115-126.

Ruchin, Peter E., et al. "Use of multiple overlapping sirolimus-eluting stents for treatment of long coronary artery lesions: Results from a single-center registry in 318 consecutive patients." International Journal of Cardiology, 2008.

Russell, James C., and Spencer, D. Proctor. "Small animal models of cardiovascular disease: tools for the study of the roles of metabolic syndrome, dyslipidemia, and atherosclerosis." Cardiovascular Pathology 15 (2006): 318-330.

Ruygrok, Peter. "Intracoronary stenting: From concept to custom." The Asia Pacific Heart Journal 6, no. 1 (May 1997): 69-70. 
Saeed, Bilal, Subhash Banerjee, and Emmanouil Brilakis. "Percutaneous Coronary Intervention in Tortuous Coronary Arteries." Journal of Interventional Cardiology, August 2008: 1-8.

Sangiorg, Giuseppe, et al. "Engineering aspects of stents design and their translation into clinical practice." Ann Ist Super Sanità 43, no. 1 (2007): 89-100.

Schoenhagen, Paul, and Steven Nissen. "Understanding coronary artery disease: tomographic imaging with intravascular ultrasound." Heart 88 (July 2002): 91-96.

Schwartz, Robert S., Nicolas A. Chronos, and Renu Virmani. "Preclinical Restenosis Models and Drug-Eluting Stents." Journal of the American College of Cardiology 44, no. 7 (2004): 1373-1385.

Scott, Neal A. "Restenosis following implantation of bare metal coronary stents: Pathophysiology and pathways involved in the vascular response to injury." Advanced Drug Delivery Reviews 58, no. 3 (2006): 358-376.

Serruys, Patrick W., and Anthony H. Gershlick. Handbook of Drug-Eluting Stents. London and New York: Taylor \& Francis, 2005.

Sharkawi, Tahmer, Frederick Cornhill, Antoine Lafont, Patrick Sabaria, and Michel Vert. "Intravascular Bioresorbable Polymeric Stents: A Potential Alternative to Current Drug Eluting Metal Stents." Journal of Pharmaceutical Sciences 96, no. 11 (November 2007): 2829-2837.

Sigwart, Ulrich, Jacques Puel, Velimir Mirkovitch, Florent Joffre, and Lukas Kappenberger. "Intravascular stents to prevent occlusion and restenosis after transluminal angioplasty." The New England Journal of Medicine 316, no. 12 (March 1987): 701-706.

Silverman M. E., Kounis N. G., Kounis G. N., Kouni S. N., Taggart D. P., Serruys P. W., Ong A. T. L. "Coronary-artery stents." New England Journal of Medicine 354 (May 2006): 2076-2078. 
Simpson, John B., Donald S. Baim, Edward W. Robert, and Donald C. Harrison. "A new catheter system for coronary angioplasty." The American Journal of Cardiology 49, no. 5 (1982): 1216-1222.

Singh, Harmeet, Eng Seng Ang, T. T. Lim, and Dietmar W. Hutmacher. "Flow Modeling in a Novel Non-Perfusion Conical Bioreactor." Biotechnology and Bioengineering 97, no. 5 (August 2007): 1291-1299.

Smith, Sidney, et al. "ACC/AHA Guidelines for Percutaneous Coronary Intervention (Revision of the 1993 PTCA Guidelines) A Report of the American College of Cardiology/American Heart Association Task Force on Practice Guidelines." Journal of the American College of Cardiology (American College of Cardiology and the American Heart Association, Inc.) 37, no. 8 (June 2001): 2215-38.

Steingruber, I. E., et al. "Technical and clinical success of infrarenal endovascular abdominal aortic aneurysm repair: A 10-year single-center experience." European Journal of Radiology 59, no. 3 (2006): 384-392.

Stella, Pieter, Ralf Mueller, George Pavlakis, Bernard Bruyne, Karl Hauptmann, and Marie-Calude Morice. "One year results of a new in-situ length-adjustable stent platform with a biodegradable Biolimus A9 eluting polymer: result of the CUSTOM-II trial." EuroIntervention 4 (2008): 200-207.

Stoll, Guido, and Martin Bendszus. "Inflammation and Atherosclerosis: Novel Insights Into Plaque Formation and Destabilization." Stroke: American Heart Association 37 (July 2006): 1923-1932.

Stoller, M. L., B. F. Schwartz, J. R. Frigstad, L. Norris, J. B. Park, and M. J. Magliochetti. "An in vitro assessment of the flow characteristics of spiral-ridged and smooth-walled JJ ureteric stents." BJU International 85, no. 6 (2000): 628-631.

Sukhija, Rishi, Jawahar L. Mehta, and Rajesh Sachdeva. "Present Status of Coronary Bifurcation Stenting." Clinical Cardiology 31 (February 2008): 63-66. 
Sulaiman, Abdulrazzaq, et al. "In vitro non-rigid life-size model of aortic arch aneurysm for endovascular prosthesis assessment." European Journal of Cardio-Thoracic Surgery 33, no. 1 (2008): 53-57.

Sun, Tao, Simon Jackson, John W. Haycock, and Sheila MacNeil. "Culture of skin cells in $3 \mathrm{D}$ rather than $2 \mathrm{D}$ improves their ability to survive exposure to cytotoxic agents." Journal of Biotechnology 122 (2006): 372-381.

Sun, Zhonghua, and Wen Jiang. "Diagnostic value of multislice computed tomography angiography in coronary artery disease: A meta-analysis." European Journal of Radiology 60 (2006): 279-286.

Szymanski, Michael, Eleni Metaxa, Hui Meng, and John Kolega. "Endothelial Cell Layer Subjected to Impinging Flow Mimicking the Apex of an Arterial Bifurcation." Annals of Biomedical Engineering 36, no. 10 (October 2008): 1681-1689.

Takeshita, Satoshi, Takaaki Shiono, Ayumu Takagi, Takenari Ito, and Shigeru Saito. "Percutaneous Coronary Intervention Using a Novel 4-French Coronary Accessor." Catheterization and Cardiovascular Interventions 72 (2008): 222-227.

Touchard, Arturo, and Robert Schwartz. "Preclinical Restenosis Models: Challenges and Successes." Toxicologic Pathology 34 (2006): 11-18.

Tunney, Michael M., Patrick F. Keane, and Sean P. Gorman. "Assessment of urinary tract biomaterial encrustation using a modified Robbins device continuous flow model." Journal of Biomedical Materials Research Part B: Applied Biomaterials 38, no. 2 (1997): 87-93.

Umashankar, P. R., P. R. Hari, and K. Sreenivasan. "Effect of blood flow on drug release from DES: An experimental study." International Journal of Cardiology, 2007.

vom Dahl, Jürgen, et al. "Effects of gold coating of coronary stents on neointimal proliferation following stent implantation." The American Journal of Cardiology 89, no. 7 (April 2002): 801-805. 
Wahle, Andreas, et al. "Plaque development, vessel curvature, and wall shear stress in coronary arteries assessed by X-ray angiography and intravascular ultrasound." Medical Image Analysis 10 (2006): 615-631.

Waksman, Ron. "Drug-eluting stents from bench to bed." Cardiovascular Radiation Medicine 3 (2002): 226-241.

Waksman, Ron. "Promise and Challenges of Bioabsorbable Stents." Catheterization and Cardiovascular Interventions 70 (2007): 407-414.

Waksman, Ron, Rajbabu Pakala, Richard Baffour, Rufus Seaborn, David Hellinga, and Fermin O. Tio. "Short-Term Effects of Biocorrodible Iron Stents in Porcine Coronary Arteries." Journal of Interventional Cardiology 21, no. 1 (2008): 15-20.

Wall, R. J., and M. Shani. "Are animal models as good as we think?" Theriogenology 69 (2008): 2-9.

Weintraub, William S. "The Pathophysiology and Burden of Restenosis." The American Journal of Cardiology 100, no. 5 (2007): S3-S9.

Weyand, Michael, Sebastian Kerber, Christof Schmid, and Norbert Rolf. "Coronary Artery Bypass Grafting With an Expanded Polytetrafluoroethylene Graft." The Annals of Thoracic Surgery 67, no. 5 (May 1999): 1240-1244.

Wieneke, H., et al. "Evaluation of Coronary Stents in the Animal Model: A Review." Materialwissenschaft und Werkstofftechnik 30, no. 12 (1999): 809-813.

Wieneke, Heinrich, et al. "Synergistic effects of a novel nanoporous stent coating and tacrolimus on intima proliferation in rabbits." Catheterization and Cardiovascular Interventions 60, no. 3 (October 2003): 399-407.

Wignall, Geoffrey R., et al. "Effects of novel coatings inspired by marine mussels on ureteral stent encrustation and uropathogen adherence in vivo." The Journal of Urology 179, no. 4 (2008): 85.

Williams, D. F. Definitions in Biomaterials. Amsterdam: Elsevier Science, 1987. 
Xu, Yan, Hui Zhang, Hao Li, and Guangshu Hu. "An improved algorithm for vessel centerline tracking in coronary angiograms." Computer Methods and Programs in Biomedicine 88 (2007): 131-143.

Yamasaki, Masao, et al. "Novel Guidewire-Based Stent Delivery System: Examination by Intravascular Ultrasound." Catheterization and Cardiovascular Interventions 72 (2008): 47-51.

Yliperttula, Marjo, Bong Geun Chung, Akshay Navaladi, Amir Manbachi, and Arto Urtti. "High-throughput screening of cell responses to biomaterials." European Journal of Pharmaceutical Sciences 35, no. 3 (2008): 151-160.

Zhu, Hui, Zhaohua Ding, Robert N. Piana, Thomas R. Gehrig, and Morton H. Friedma. "Cataloguing the geometry of the human coronary arteries: A potential tool for predicting risk of coronary artery disease." International Journal of Cardiology, 2008.

Zoghbi, Gilbert J., Todd A. Dorfman, and Ami E. Iskandrian. "The Effects of Medications on Myocardial Perfusion." Journal of the American College of Cardiology 52, no. 6 (August 2008): 401-416. 
AAA - Abdominal Aortic Aneurysm

ACC - American College of Cardiology

AHA - American Heart Association

BMS - Bare Metal Stent

BVM - Blood Vessel Mimic

CABG - Coronary Artery Bypass Graft

CAD - Coronary Artery Disease

CHD - Coronary Heart Disease

CT - Computerized Tomography

DES - Drug Eluting Stent

EC - Endothelial Cells

ECG/EKG - Electrocardiogram

ECHO - Echocardiogram

EO - Ethylene Oxide

ePTFE - Expanded Polytetrafluoroethylene

EVAR - Endovascular Aortic Aneurysm Repair

HA - Hyaluronic Acid

HUASMC - Human Umbilical Artery Smooth Muscle Cells

ID - Inner Diameter

ISR - In-stent Restenosis

IVUS - Intravascular Ultrasound

MRI - Magnetic Resonance Imaging

OD - Outer Diameter

PBMA - Poly-n-butyl Methacrylate

PC - Phosphorylcholine

PCI - Percutaneous Coronary Intervention

PCL - Polycaprolactone

PDLA - Poly-D,L-lactic Acid

PET - Positron Emission Tomography

PEVA - Polyethylene-co-vinyl Acetate

PGA - Polyglycolic Acid

PLGA - Polylactic-co-glycolic Acid

PLLA - Poly-L-lactic Acid

$\mathrm{P} / \mathrm{N}$ - Part Number

PTCA - Percutaneous Transluminal Coronary Angioplasty

SEM - Scanning Electron Microscope

SMC - Smooth Muscle Cells

TEBV - Tissue Engineered Blood Vessels

VSMC - Vascular Smooth Muscle Cells

W - Width 


\section{APPENDIX B: PART INFORMATION AND DRAWINGS}

\begin{tabular}{|c|c|c|c|c|}
\hline Component & Description & Company & Website & Part Number \\
\hline Chamber & $\begin{array}{l}\text { Polypropylene Leak- } \\
\text { Proof Container, } \\
\text { 5"x4"x2", } 350 \mathrm{ml}\end{array}$ & $\begin{array}{c}\text { Lock \& } \\
\text { Lock }\end{array}$ & www.locknlock.com.au & HPL806 \\
\hline Chamber & $\begin{array}{l}\text { Polypropylene Leak- } \\
\text { Proof Container, } \\
8 \text { "x5"x2", } 800 \mathrm{ml}\end{array}$ & $\begin{array}{c}\text { Lock \& } \\
\text { Lock }\end{array}$ & www.locknlock.com.au & HPL816 \\
\hline $\begin{array}{l}\text { Buna-N } \\
\text { O-Ring }\end{array}$ & $\begin{array}{c}\text { Soft Buna-N O-Ring } \\
\text { AS568A Dash } \\
\text { Number 006 }\end{array}$ & $\begin{array}{c}\text { McMaster- } \\
\text { Carr }\end{array}$ & www.mcmaster.com & $2418 \mathrm{~T} 112$ \\
\hline Loctite 4011 & $\begin{array}{c}\text { Loctite Prism } 4011 \\
\text { Medical Adhesive, } 20 \\
\text { Gram Bottle }\end{array}$ & $\begin{array}{c}\text { McMaster- } \\
\text { Carr }\end{array}$ & www.mcmaster.com & 1818A4 \\
\hline $\begin{array}{l}\text { Female Luer } \\
\text { Lug Panel } \\
\text { Mount-1/8" } \\
\text { Barb }\end{array}$ & $\begin{array}{l}\text { Female Luer Lug Style } \\
\text { Panel Mount 1/4-28 } \\
\text { UNF to } 200 \text { Series Barb, } \\
\text { 1/8" (3.2 mm) ID } \\
\text { Tubing, White Nylon }\end{array}$ & $\begin{array}{c}\text { Value } \\
\text { Plastics }\end{array}$ & www.valueplastics.com & FTLB230-1 \\
\hline Lock Nut & $\begin{array}{l}\text { 1/4-28 UNF Panel } \\
\text { Mount Lock Nut, } \\
\text { White Nylon }\end{array}$ & $\begin{array}{c}\text { Value } \\
\text { Plastics }\end{array}$ & www.valueplastics.com & LNS-1 \\
\hline $\begin{array}{l}\text { Male Luer } \\
\text { Lock-1/8" } \\
\text { Barb }\end{array}$ & $\begin{array}{l}\text { Male Luer Integral Lock } \\
\text { Ring to } 200 \text { Series Barb, } \\
\text { 1/8" (3.2 mm) ID } \\
\text { Tubing, White Nylon }\end{array}$ & $\begin{array}{c}\text { Value } \\
\text { Plastics }\end{array}$ & www.valueplastics.com & MTLL230-1 \\
\hline $\begin{array}{l}\text { Female Luer } \\
\text { Thread-1/8" } \\
\text { Barb }\end{array}$ & $\begin{array}{c}\text { Female Luer Thread } \\
\text { Style to } 200 \text { Series Barb, } \\
\text { 1/8" (3.2 mm) ID } \\
\text { Tubing, White Nylon }\end{array}$ & $\begin{array}{c}\text { Value } \\
\text { Plastics }\end{array}$ & www.valueplastics.com & FTLL230-1 \\
\hline $\begin{array}{l}\text { Female Luer } \\
\text { Thread-3/32" } \\
\text { Barb }\end{array}$ & $\begin{array}{l}\text { Female Luer Thread } \\
\text { Style to } 200 \text { Series Barb, } \\
\text { 3/32" (2.25 mm) ID } \\
\text { Tubing, White Nylon }\end{array}$ & $\begin{array}{c}\text { Value } \\
\text { Plastics }\end{array}$ & www.valueplastics.com & FTLL220-1 \\
\hline $\begin{array}{c}\text { Male-Female } \\
\text { Coupler }\end{array}$ & $\begin{array}{c}\text { Male Luer Integral Lock } \\
\text { Ring to Female Luer } \\
\text { Thread Style Coupler, } \\
\text { White Nylon }\end{array}$ & $\begin{array}{c}\text { Value } \\
\text { Plastics }\end{array}$ & www.valueplastics.com & LC34-1 \\
\hline $\begin{array}{l}\text { Y-Connector- } \\
\text { 1/8" Barbs }\end{array}$ & $\begin{array}{c}\text { Y-Connector with } 200 \\
\text { Series Barbs, 1/8" (3.2 } \\
\text { mm) ID Tubing, } \\
\text { White Nylon }\end{array}$ & $\begin{array}{c}\text { Value } \\
\text { Plastics }\end{array}$ & www.valueplastics.com & Y230-1 \\
\hline $\begin{array}{c}\text { 1-Way } \\
\text { Stopcock }\end{array}$ & $\begin{array}{c}\text { One-Way Stopcock, } \\
\text { Capped Male Luer Lock } \\
\text { to Capped Female Luer } \\
\text { Thread Style, } \\
\text { Polycarbonate Body w/ } \\
\text { Polyethylene Handle }\end{array}$ & $\begin{array}{c}\text { Value } \\
\text { Plastics }\end{array}$ & www.valueplastics.com & VP455980 \\
\hline
\end{tabular}




\begin{tabular}{|c|c|c|c|c|}
\hline $\begin{array}{l}\text { 3-Way } \\
\text { Stopcock }\end{array}$ & $\begin{array}{l}\text { Three-Way Stopcock, } 2 \\
\text { Capped Female Luer } \\
\text { Thread Style \& Capped } \\
\text { Male Luer Lock, } \\
\text { Polycarbonate Body w/ } \\
\text { Polyethylene Handle } \\
\end{array}$ & $\begin{array}{l}\text { Value } \\
\text { Plastics }\end{array}$ & www.valueplastics.com & VPB1000079N \\
\hline Male Plug & $\begin{array}{l}\text { Male Luer Integral Lock } \\
\text { Ring Plug, Closed at } \\
\text { Grip, White Nylon }\end{array}$ & $\begin{array}{l}\text { Value } \\
\text { Plastics }\end{array}$ & www.valueplastics.com & LP4-1 \\
\hline $\begin{array}{l}\text { Male-to-Male } \\
\text { Rotating Luer }\end{array}$ & $\begin{array}{l}\text { Male-to-Male Luer } \\
\text { Connector with One } \\
\text { Rotating Collar, ABS }\end{array}$ & Qosina & www.qosina.com & 17664 \\
\hline $50 \mathrm{ml}$ Tube & $\begin{array}{l}\text { BD Falcon }{ }^{\mathrm{TM}} 50 \mathrm{ml} \\
\text { Conical-Bottom } \\
\text { Polypropylene Tube }\end{array}$ & $\begin{array}{c}\text { BD } \\
\text { Biosciences }\end{array}$ & www.bdbiosciences.com & 352098 \\
\hline $\begin{array}{l}\text { Silicone } \\
\text { Tubing }\end{array}$ & $\begin{array}{c}\text { Tygon® Silicone Tubing } \\
1 / 8 \text { " ID x } 1 / 4 " \text { OD }\end{array}$ & Cole-Parmer & www.coleparmer.com & WU-95702-06 \\
\hline
\end{tabular}




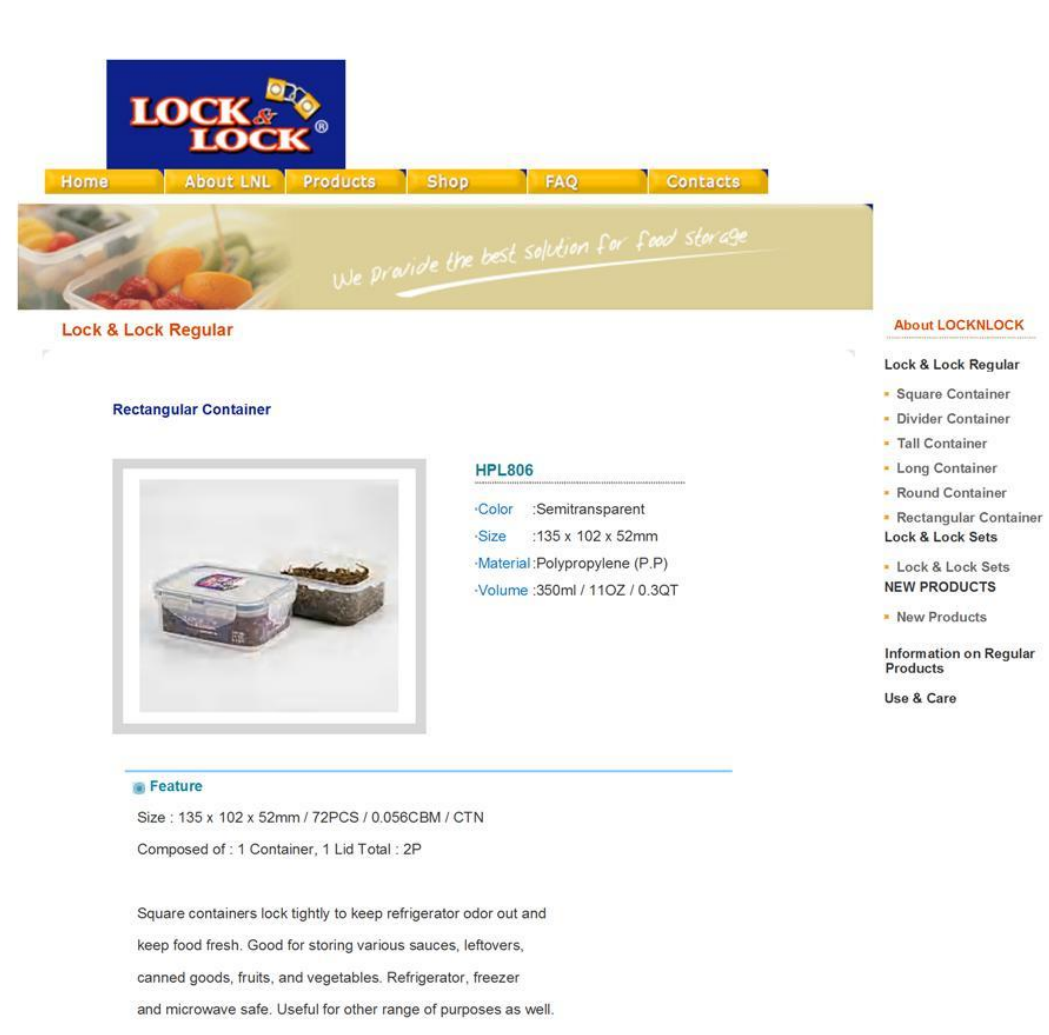




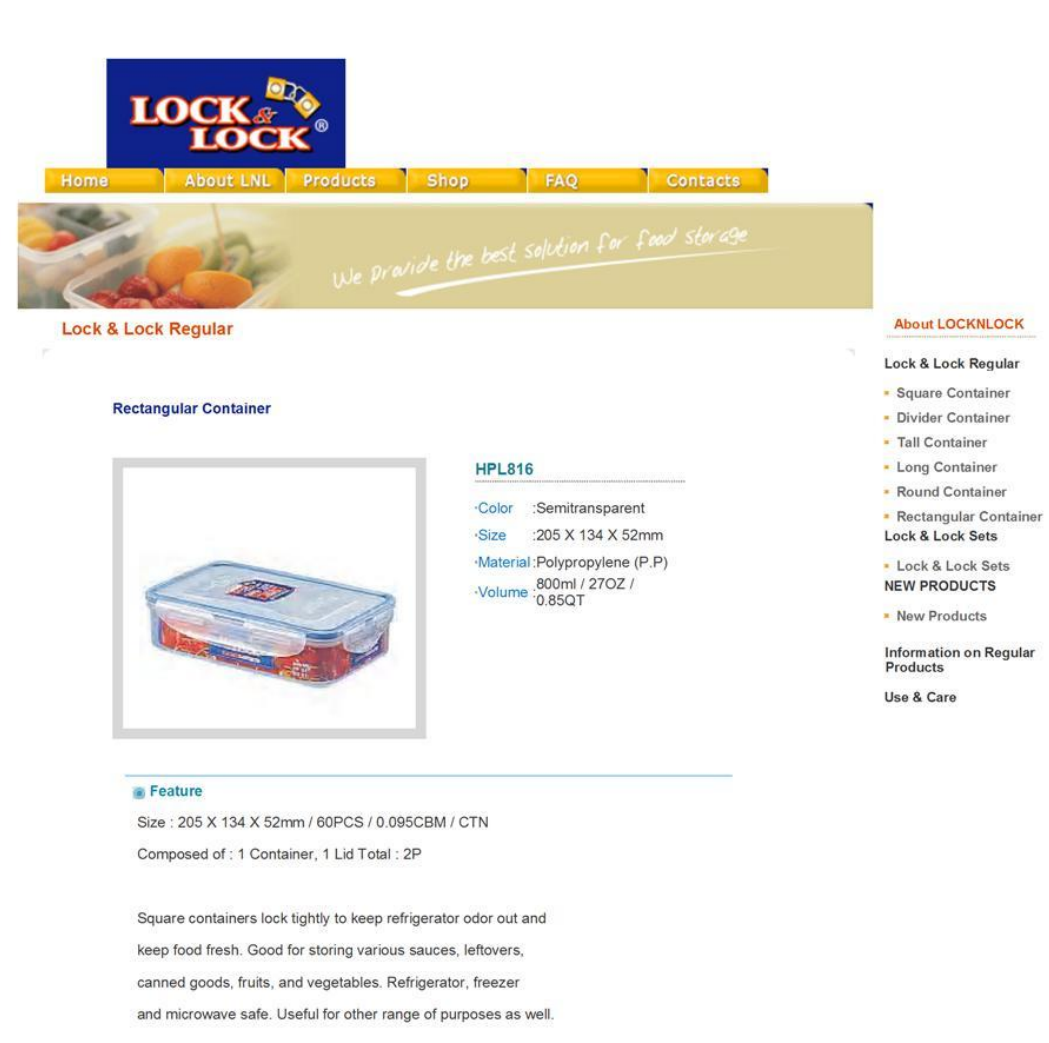




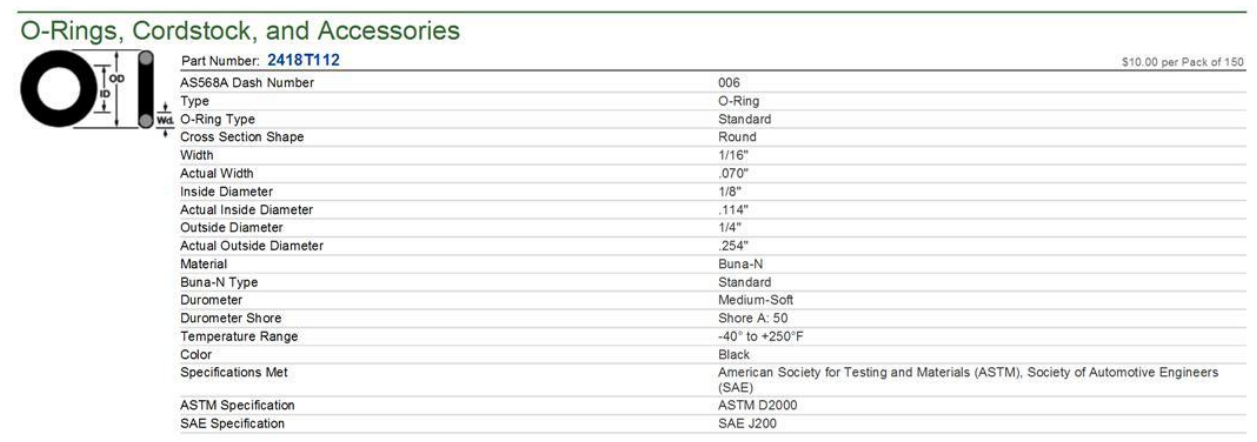




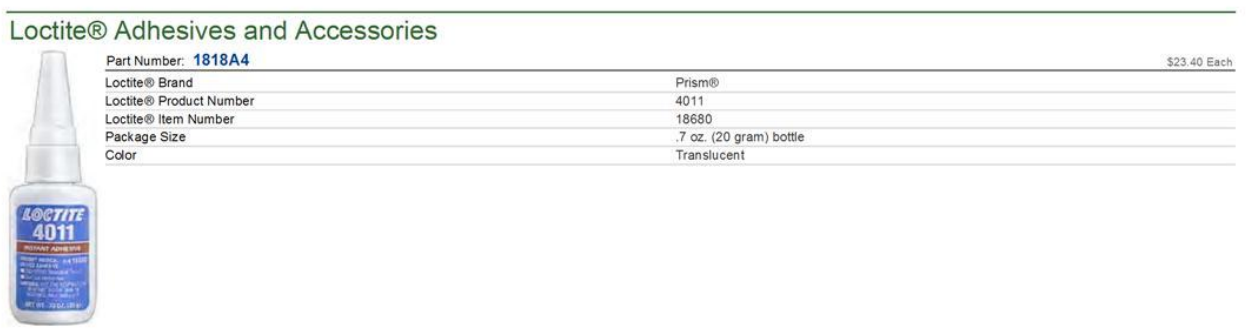




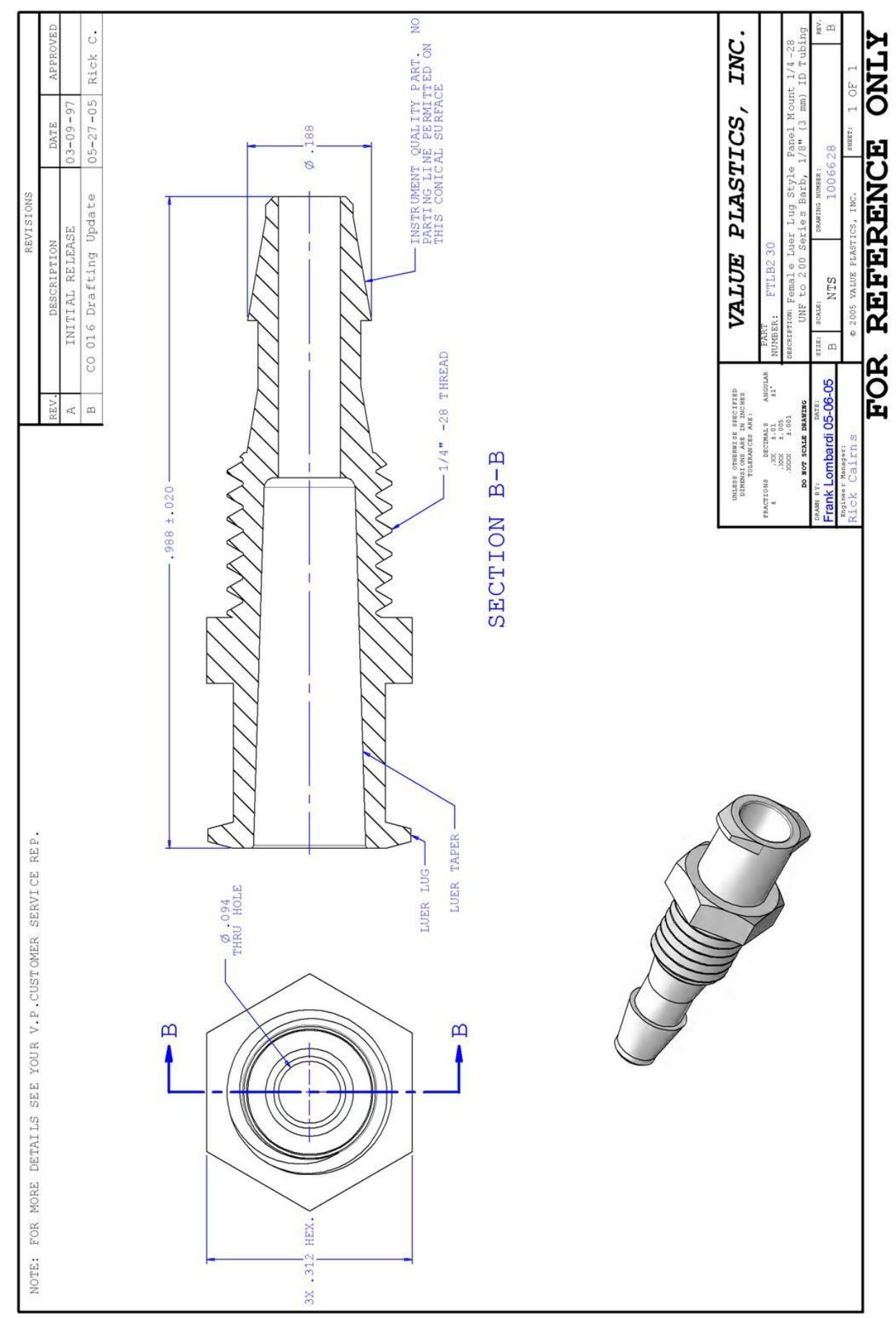




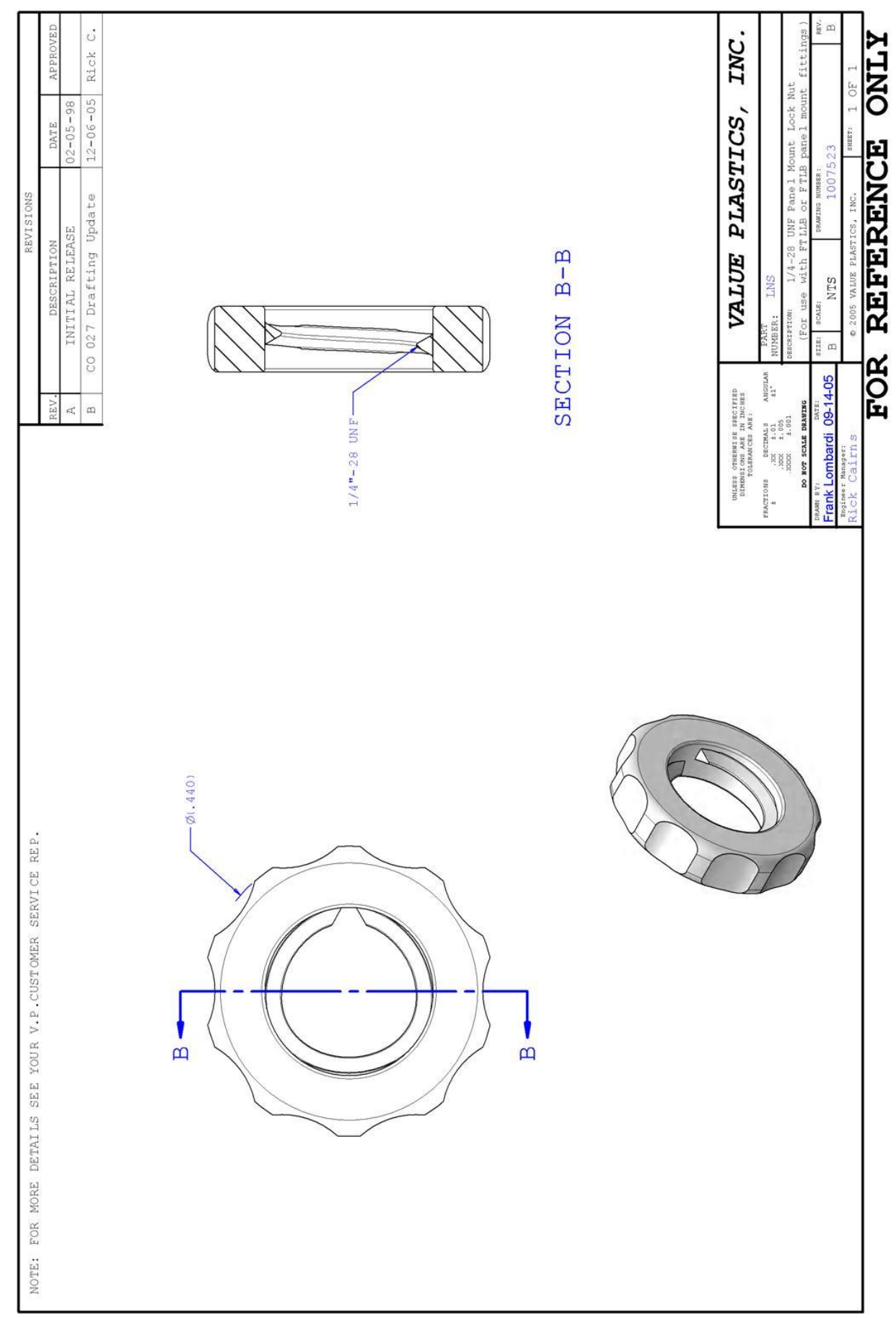




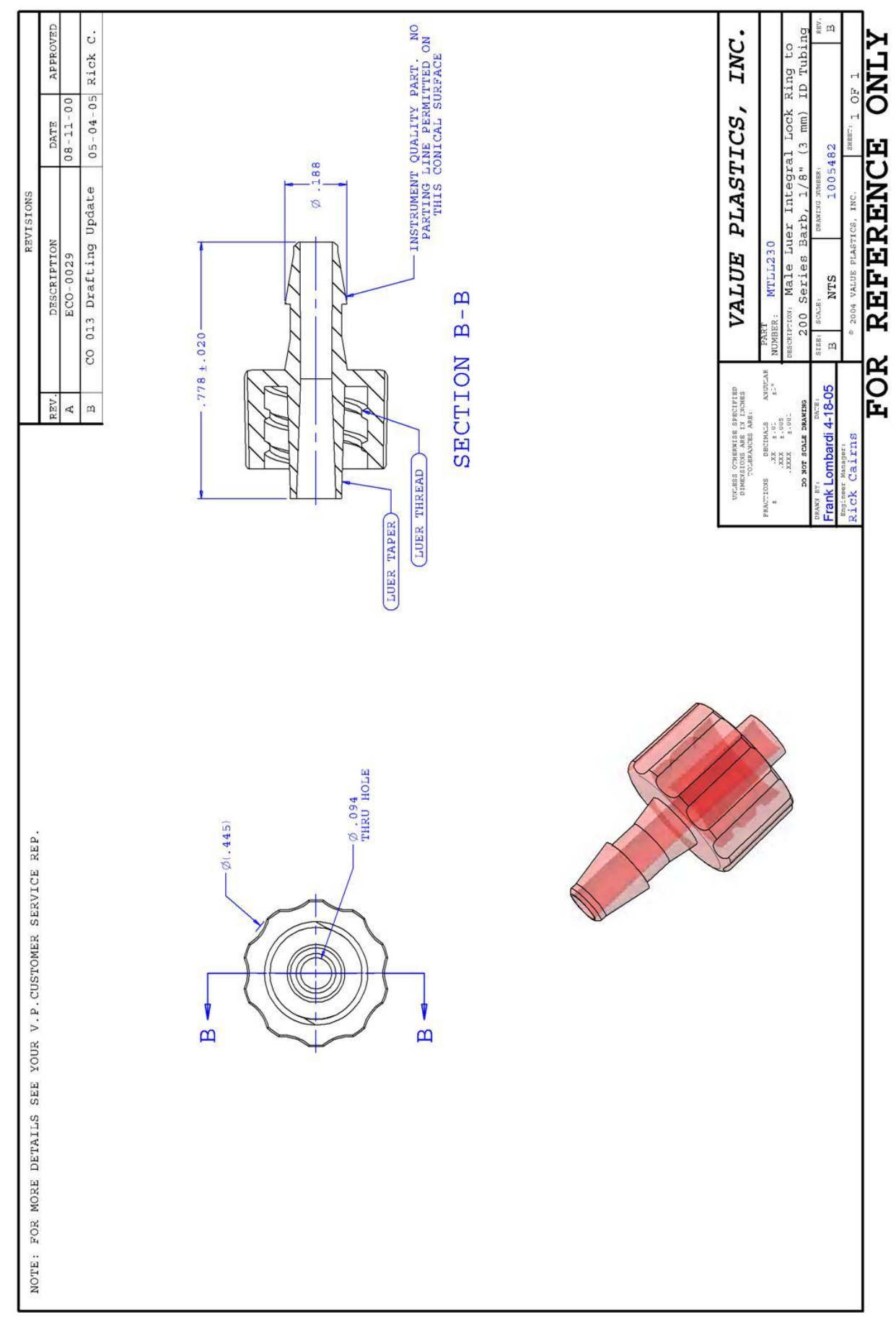




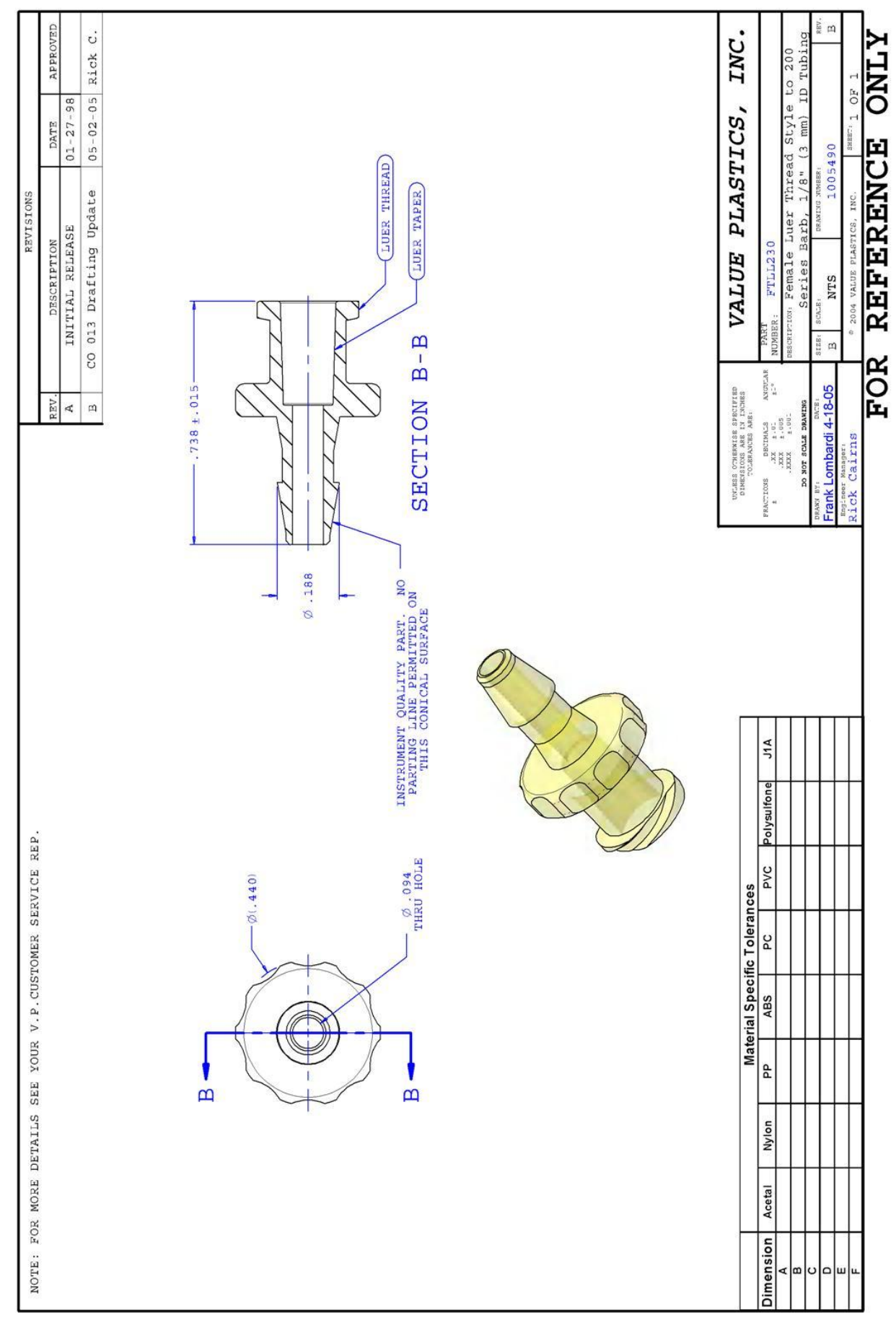




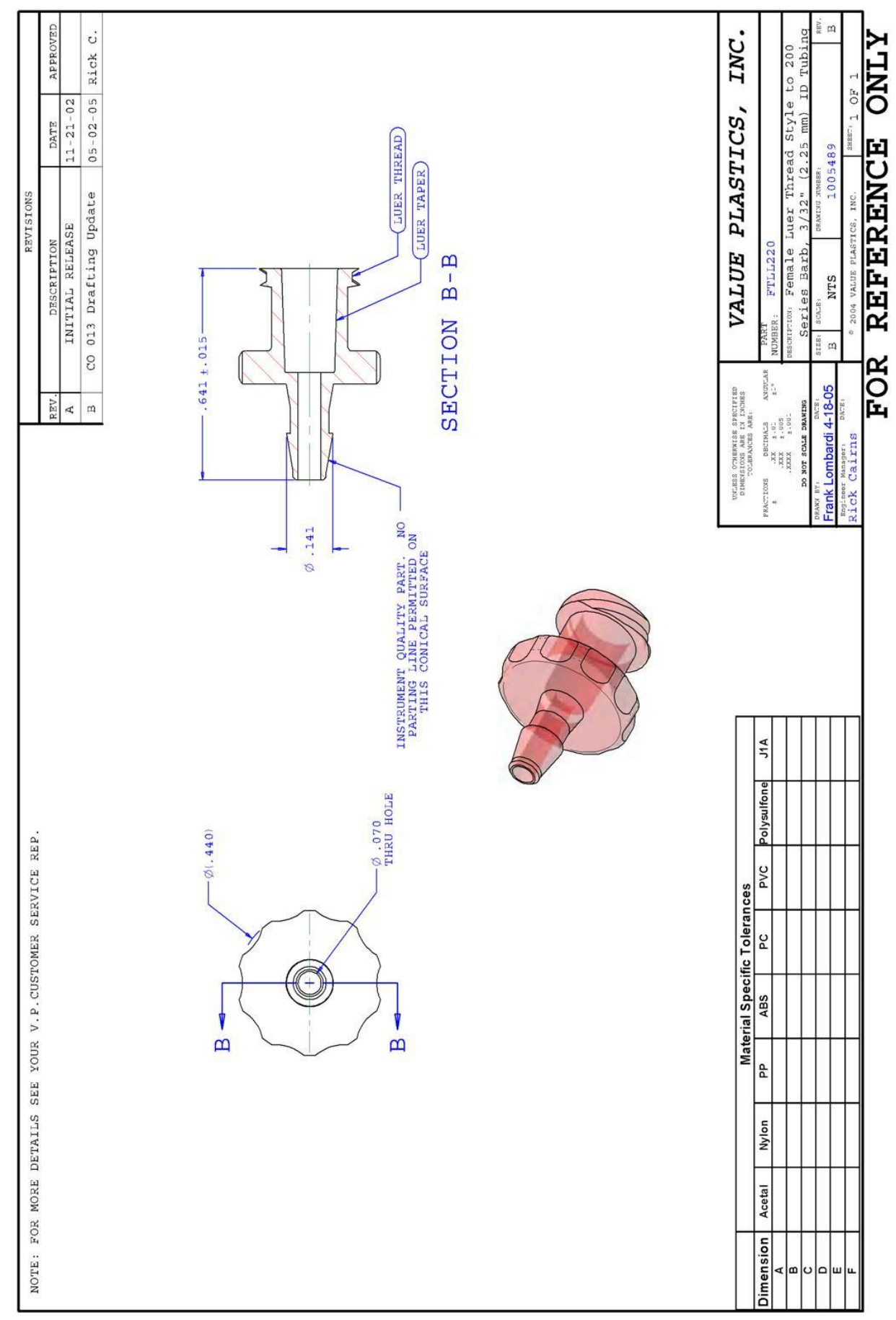




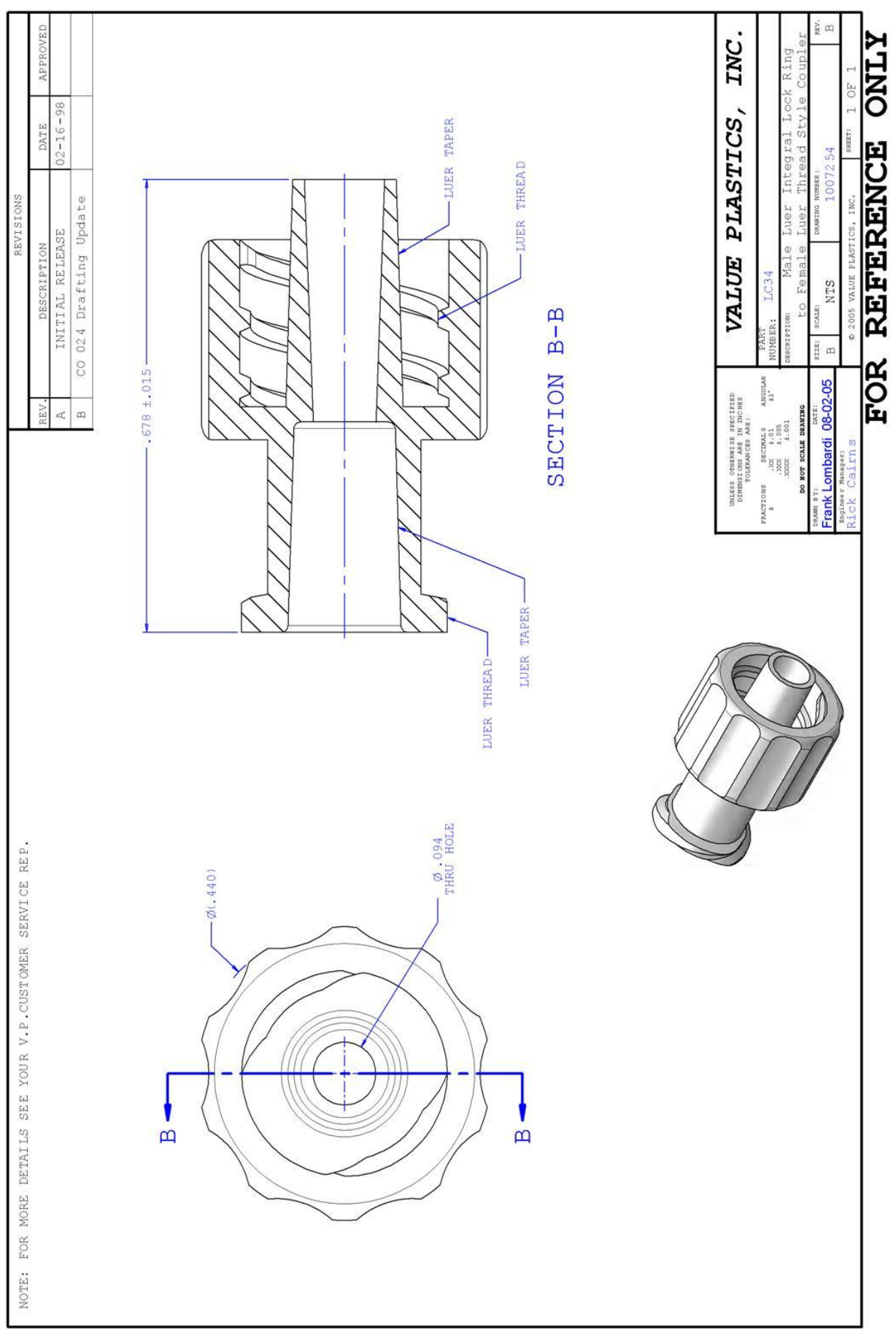




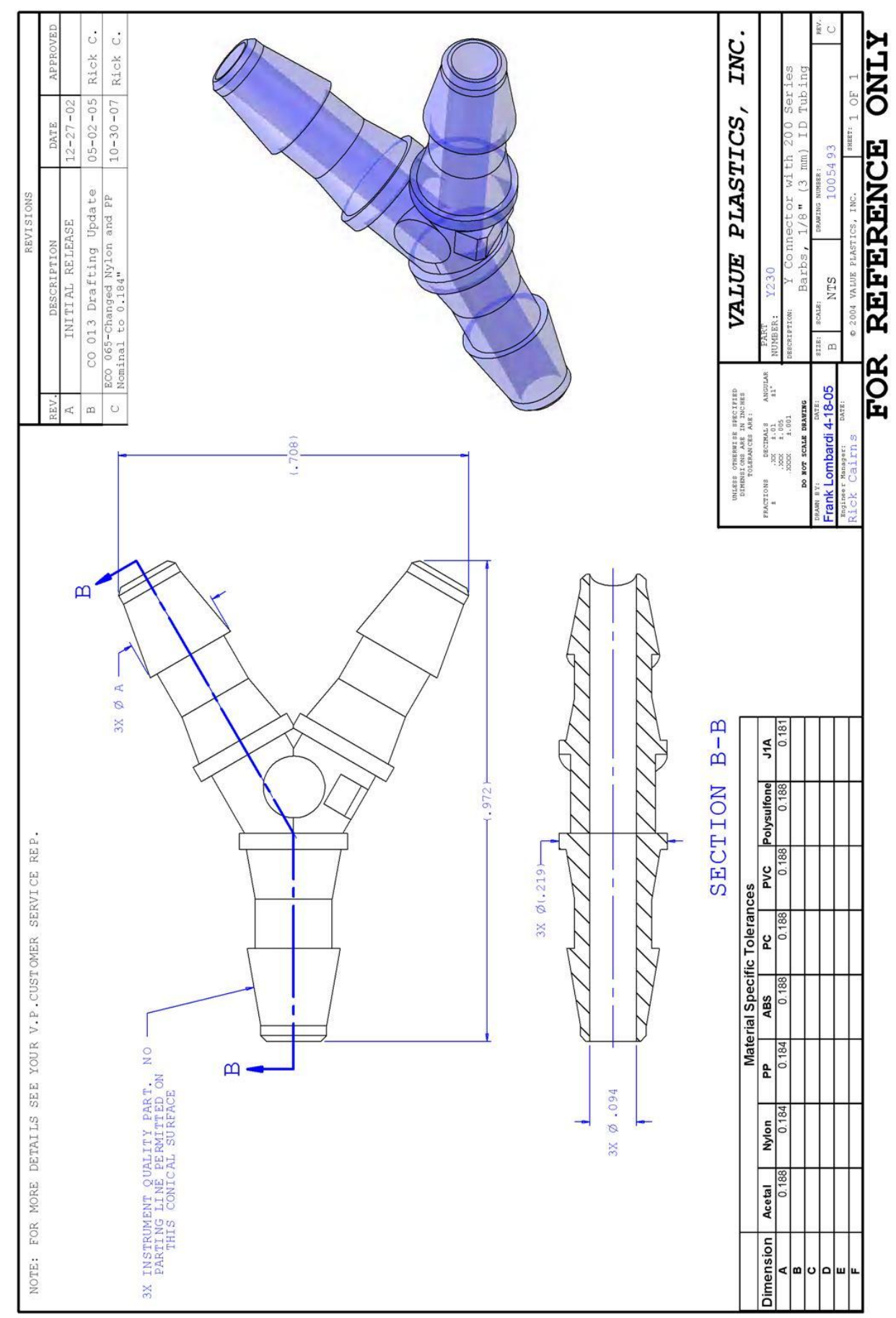




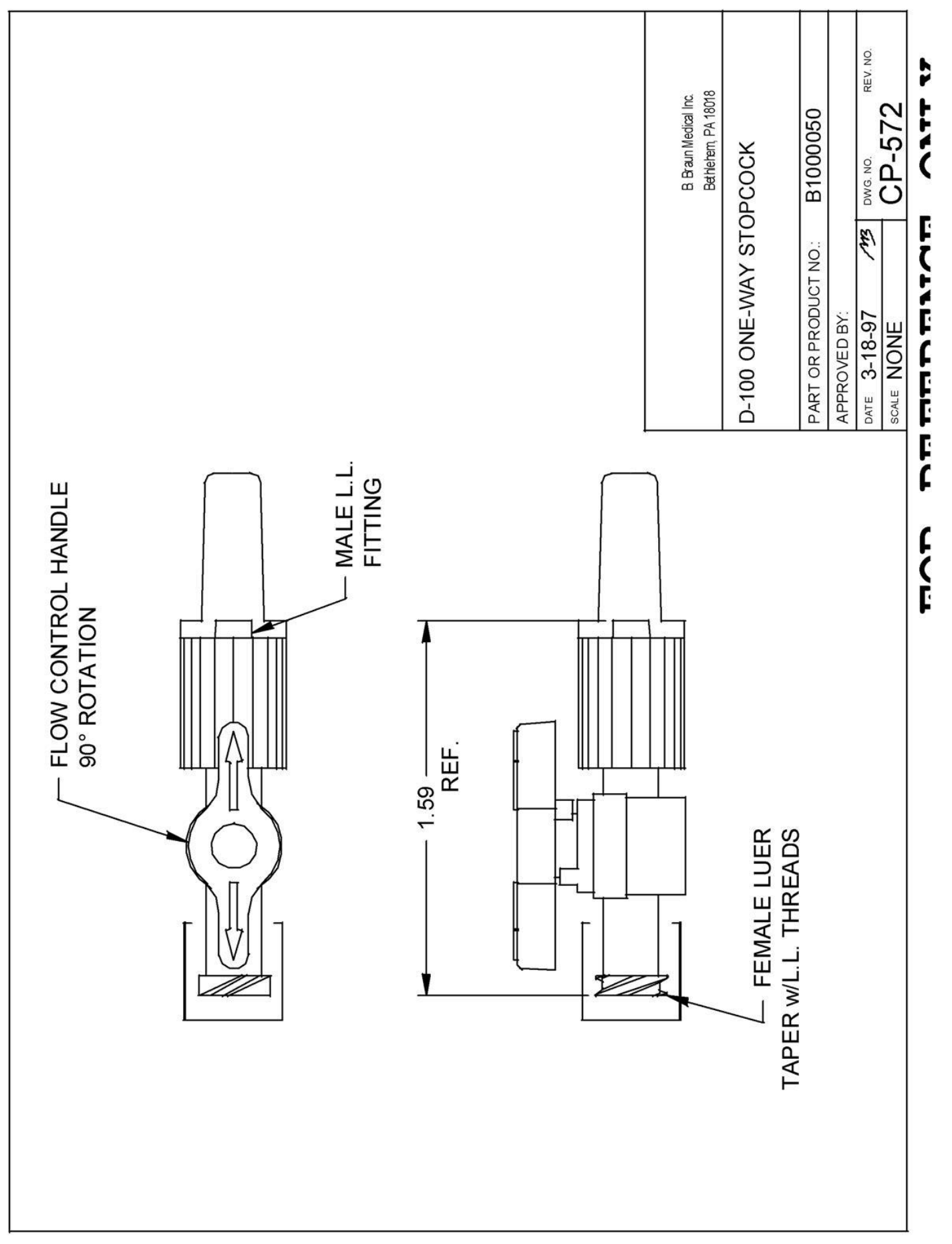




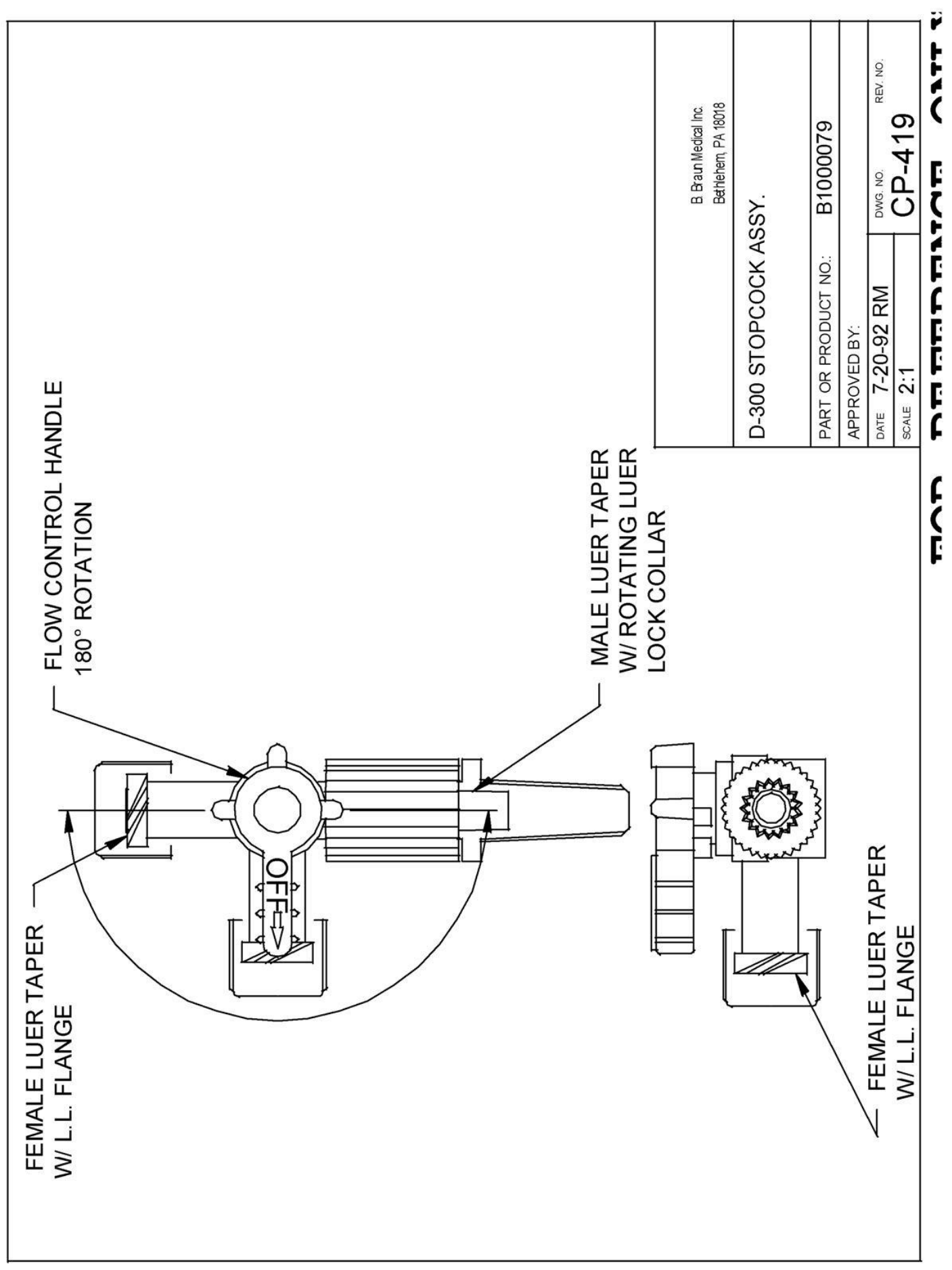




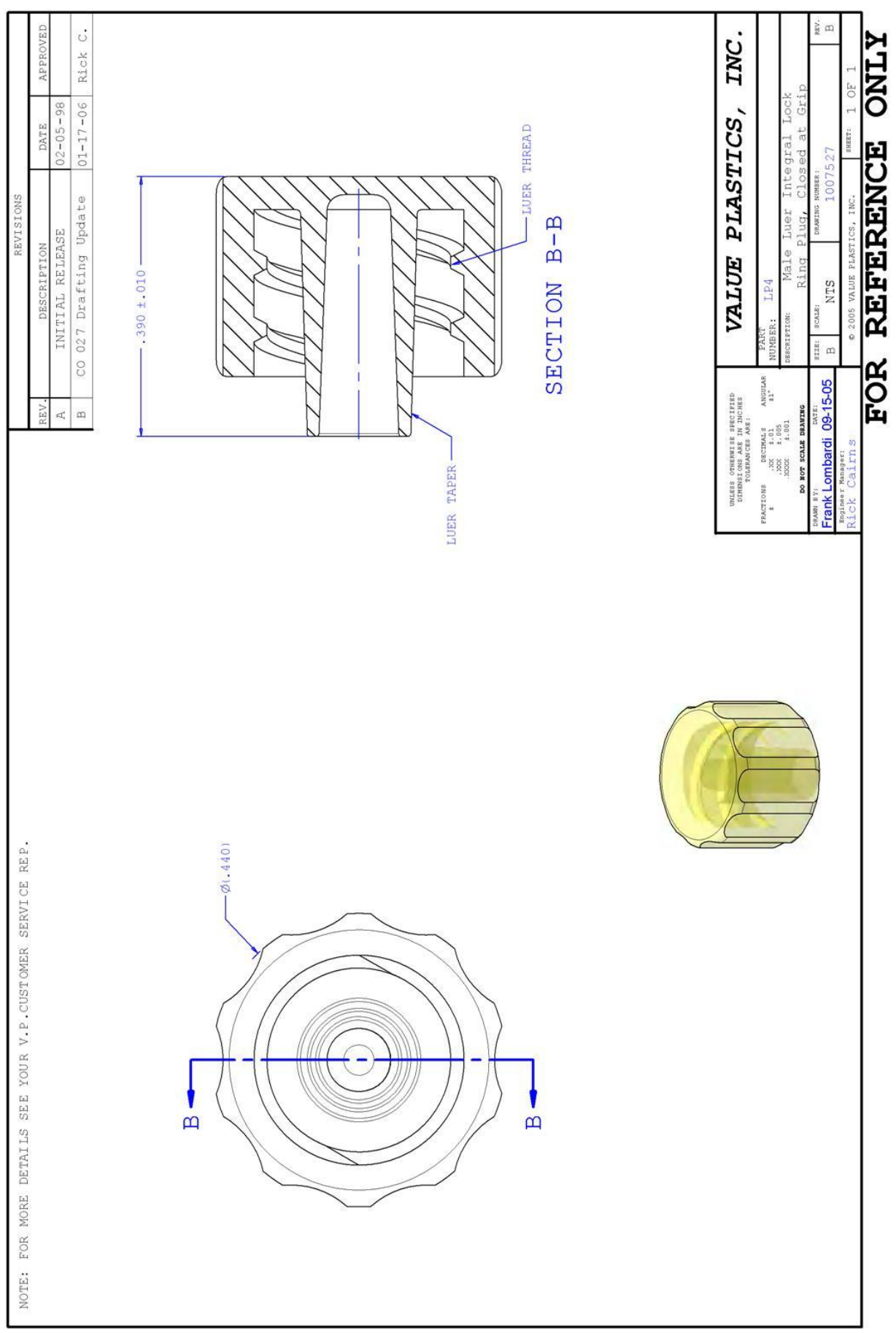




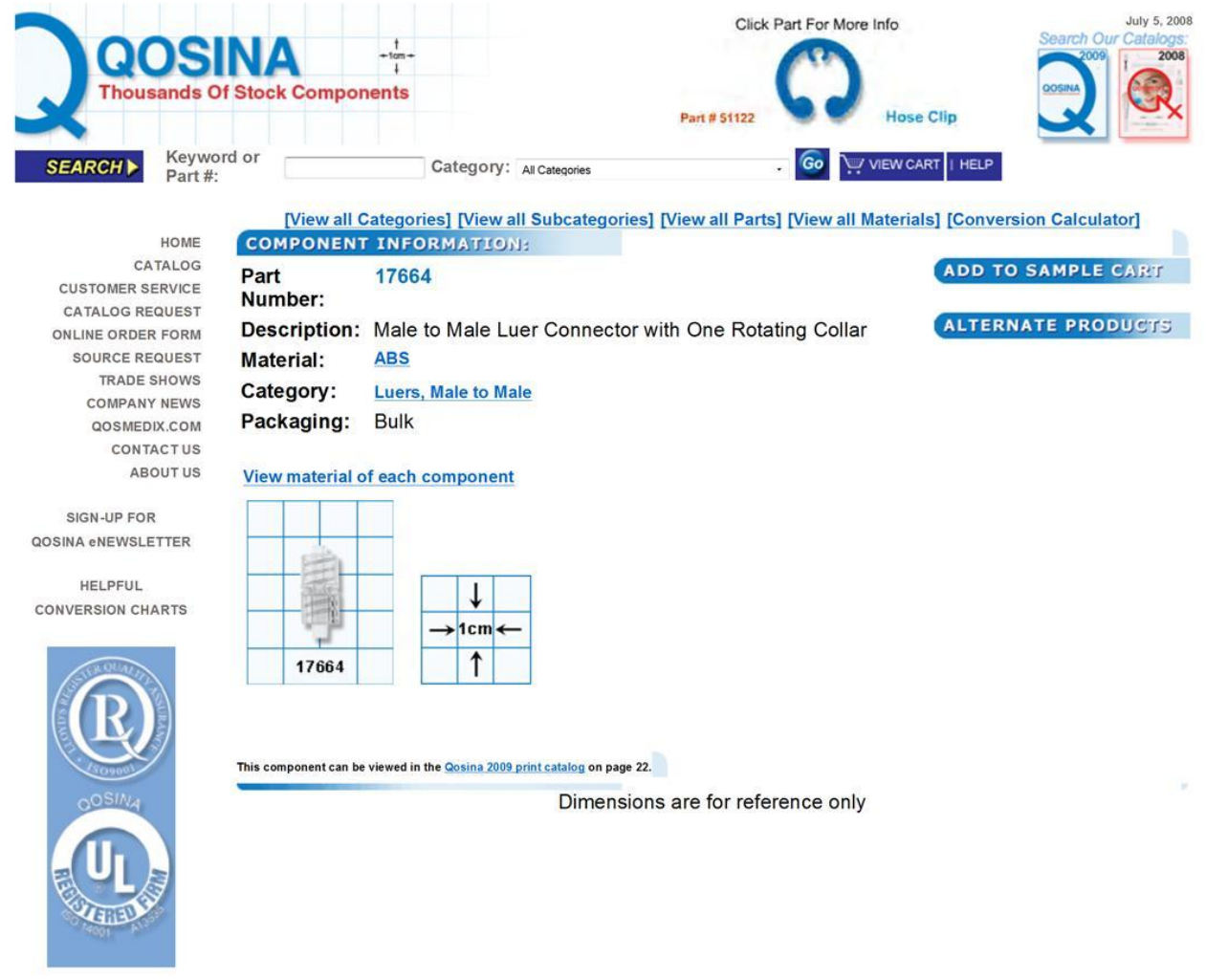




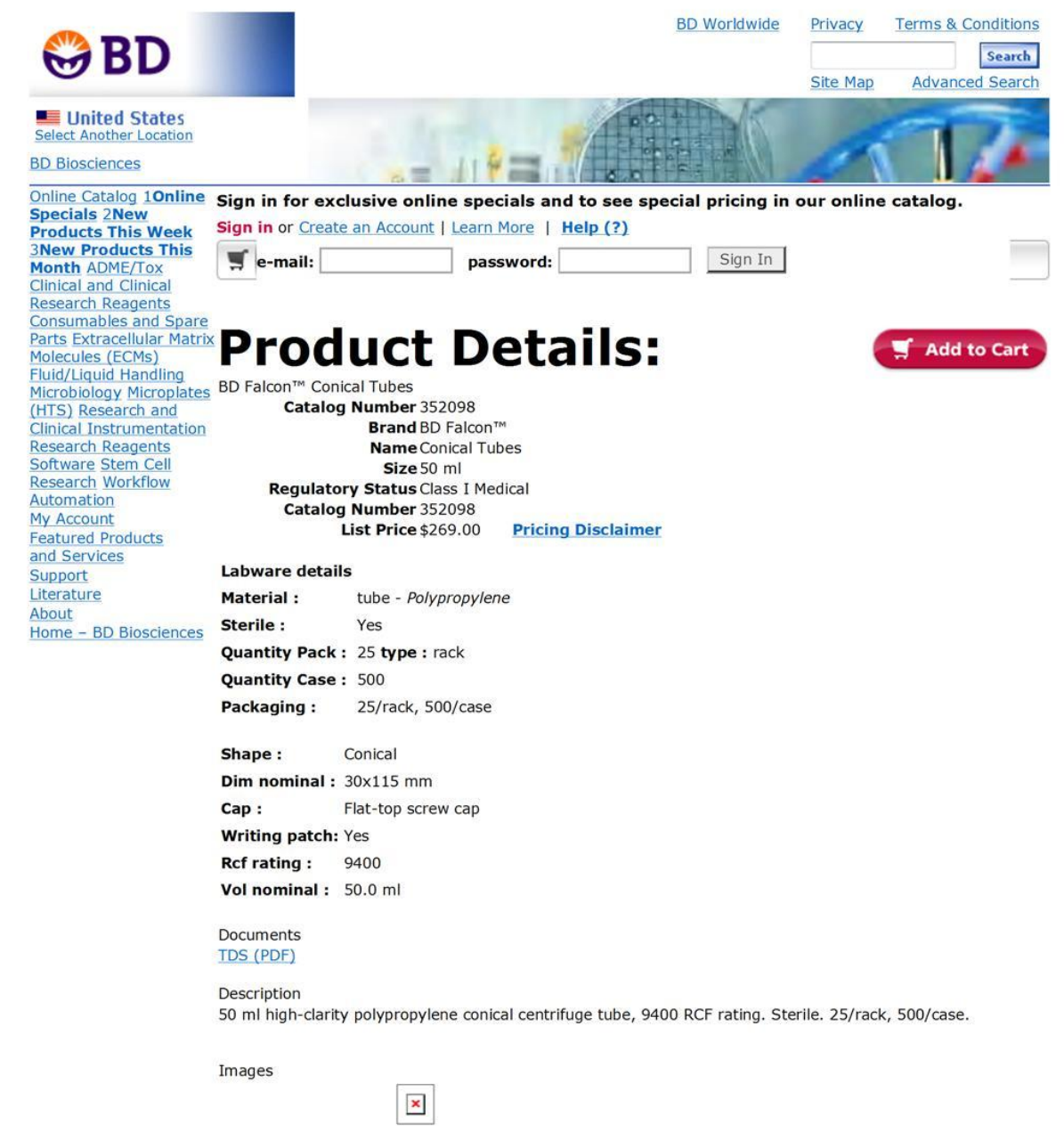

Privacy | Terms \& Conditions | Product Terms \& Conditions | Permission Request | Trademarks $\mid$ Site Map $\mid$ BD Worldwide

$\begin{array}{ll}\text { BD } & \text { BD Biosciences } \\ 1 \text { Becton Drive } & \text { 2350 Qume Drive } \\ \text { Franklin Lakes, NJ USA } 07417 & \text { San Jose, CA USA } 95131 \\ 201.847 .6800 & 877.232 .8995\end{array}$

Unless otherwise noted, BD, BD Logo and all other trademarks are the property of Becton, Dickinson and Company. (๑) 2005 BD

eCat $\mathrm{v} 4.3$ build 0701221056 


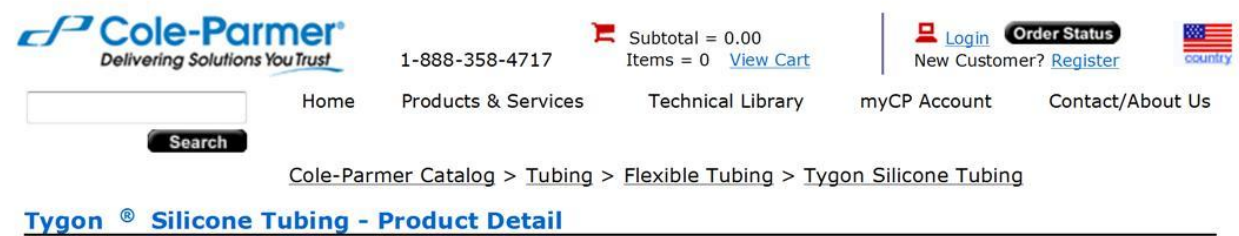

WU-95702-06 (7 of 20) [Previous । Next]

\section{Tygon ${ }^{\circledR}$ Silicone Tubing}

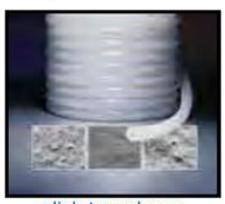

click to enlarge

WU-95702-06

(package)
Tygon ${ }^{(8)}$ silicone tubing, $1 / 8$ "ID $x$

$1 / 4 " O D, 50$ feet/pack

$\$ 109.00$ / package (USD) $S$

Click $\$$ for discounts.

Product Rating

= Find the nominal tubing you need -Quick

Formulation: TYGON 3350 silicone, platinum cured.

Applications: Pharmaceutical, cosmetic, medical, industrial, and auto-analysis applications.

Characteristics: Ultra-smooth inner surface reduces particle entrapment and minimizes bacterial growth. Good for mild to medium concentration bases, salts, and alcohols; is odorless, tasteless, and non-toxic. Transparent.

Certification: Elastomer is FDA-compliant (21 CFR 177.2600); meets USP 23 Class VI requirements, and NSF criteria. Tubing is manufactured according to GMP.

Temperature range: -112 to $392^{\circ} \mathrm{F}\left(-80\right.$ to $\left.200^{\circ} \mathrm{C}\right)$ Sterilize: With ethylene oxide, gamma irradiation, or autoclave for $30 \mathrm{~min}, 15 \mathrm{psi}$.

Specifications
\begin{tabular}{|l|c|}
\hline Material & Tygon ${ }^{\circledR}$ Silicone \\
\hline ID & $1 / 8^{\prime \prime}(3.2 \mathrm{~mm})$ \\
\hline OD & $1 / 4^{\prime \prime}(6.4 \mathrm{~mm})$ \\
\hline Wall thickness & $1 / 16^{\prime \prime}(1.6 \mathrm{~mm})$ \\
\hline Max psi & $9 \mathrm{psi}$ at $73^{\circ} \mathrm{F}$ \\
\hline Certifications & FDA, USP, NSF, GMP \\
\hline
\end{tabular}

TT Technical Appendix

- Technical Library (roll over link for article summary)

Cole-Parmer's Bioconnect Program

Decrease bacterial growth in your tubin

The "STAMP" Tubing Selection Guide

More Related TechInfo Articles

PRODUCT REVIEWS SUMMARY

Be the first to review this product. Write a Review
T) Technical Appendix

C Custom Ordering Solutions

Let us find the exact product you need.

We have access to suppliers full lines-

Call 1-800-323-4340, Option 9 , ext

6334. See details

园 Literature

国07-08 Catalog

通05-06 Catalog 
APPENDIX C: IDEAL BVM SYSTEM ASSEMBLY

\begin{tabular}{|c|c|c|c|}
\hline Component & Company & Part Number & Quantity \\
\hline Leak-Proof Chamber & Lock \& Lock & HPL806 & 1 EA \\
\hline Buna-N O-Ring & McMaster-Carr & $2418 T 112$ & 6 EA \\
\hline Loctite 4011 Adhesive & McMaster-Carr & 1818 A4 & 3 drops \\
\hline Female Luer Lug Panel Mount-1/8" Barb & Value Plastics & FTLB230-1 & 3 EA \\
\hline Lock Nut & Value Plastics & LNS-1 & 3 EA \\
\hline Male Luer Lock-1/8" Barb & Value Plastics & MTLL230-1 & 2 EA \\
\hline Female Luer Thread-1/8" Barb & Value Plastics & FTLL230-1 & 2 EA \\
\hline Y-Connector-1/8" Barbs & Value Plastics & Y230-1 & 1 EA \\
\hline Male-Female Coupler & Value Plastics & LC34-1 & 2 EA \\
\hline 1-Way Stopcock & Value Plastics & VP455980 & 2 EA \\
\hline 3-Way Stopcock & Value Plastics & VPB1000079N & 1 EA \\
\hline Male-to-Male Rotating Luer Lock & Qosina & 17664 & 2 EA \\
\hline Tygon® Silicone Tubing, 1/8" ID & Cole-Parmer & WU-95702-06 & 11 inches \\
\hline
\end{tabular}

1. Drill three 15/64" holes into the sides of the leak-proof chamber (Lock \& Lock P/N HPL806).

a. Drill the luminal inlet hole on the left face of the chamber $1 \mathrm{~cm}$ down from the top centered notch and $1 \mathrm{~cm}$ to the right.

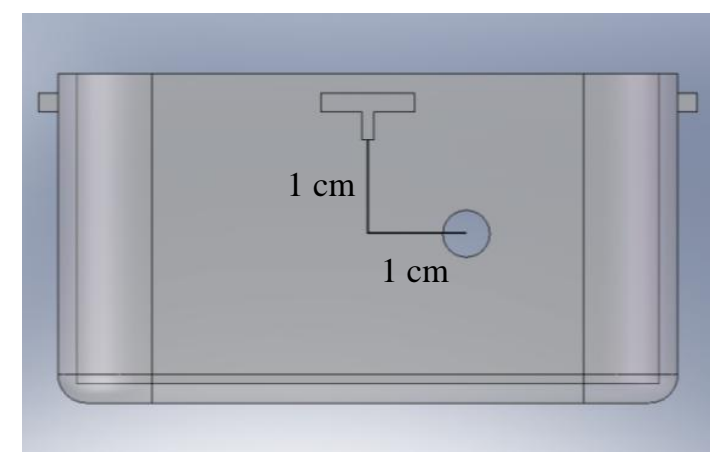


b. Drill the luminal outlet hole on the right face of the chamber $1 \mathrm{~cm}$ down from the top centered notch and $1 \mathrm{~cm}$ to the left.

c. Drill the extra luminal outlet hole on the right face of the chamber $1 \mathrm{~cm}$ down from the top centered notch and $2 \mathrm{~cm}$ to the right.

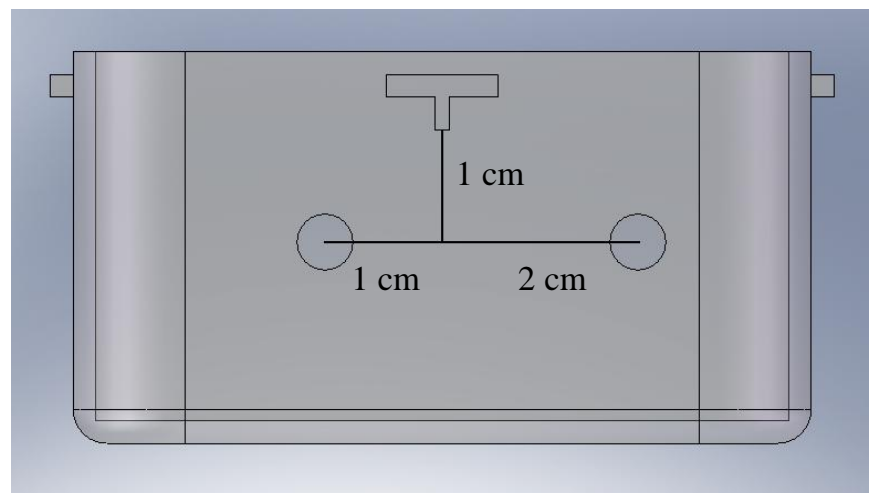

2. Using a razor blade, remove any plastic debris that resulted from the use of the drill from the edges of the holes.

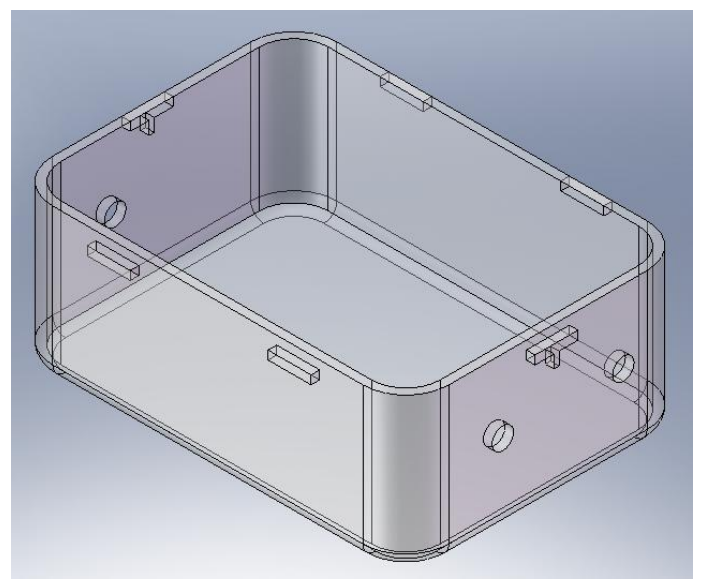

3. Slide a Buna-N o-ring (McMaster-Carr P/N 2418T112) onto a female luer lug panel mount-1/8" barb (Value Plastics P/N FTLB230-1).

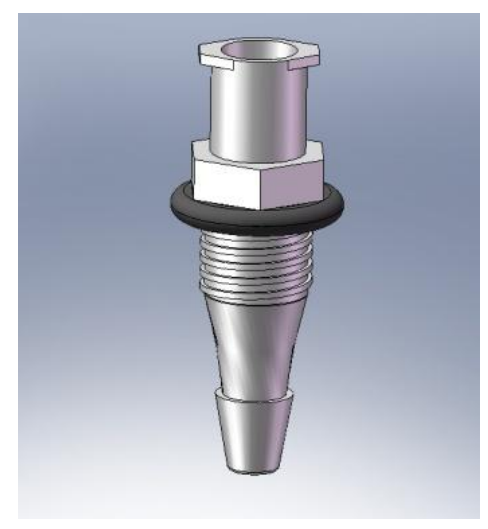


4. Thread the female luer lug panel mount with the Buna-N o-ring through the drilled luminal inlet hole with the barbed portion exiting the side of the chamber.
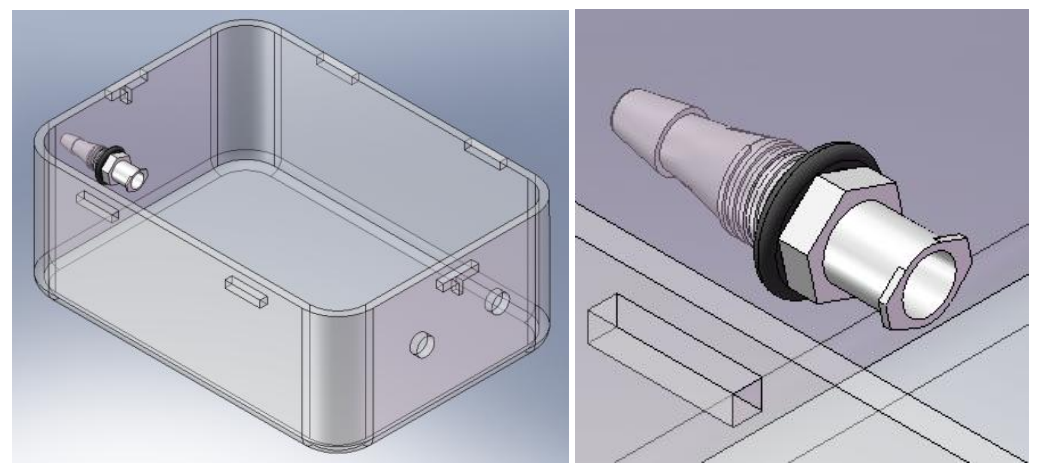

5. Slide a second Buna-N o-ring (McMaster-Carr P/N 2418T112) onto the barbed end of the female luer lug panel mount.
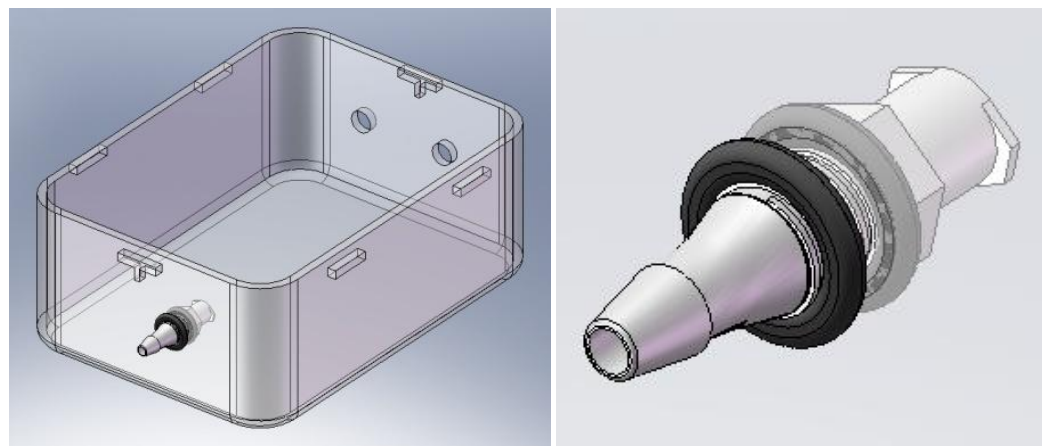

6. Tightly screw a lock nut (Value Plastics P/N LNS-1) onto the female luer lug panel mount, pressing the o-rings against the inside and outside walls of the chamber.
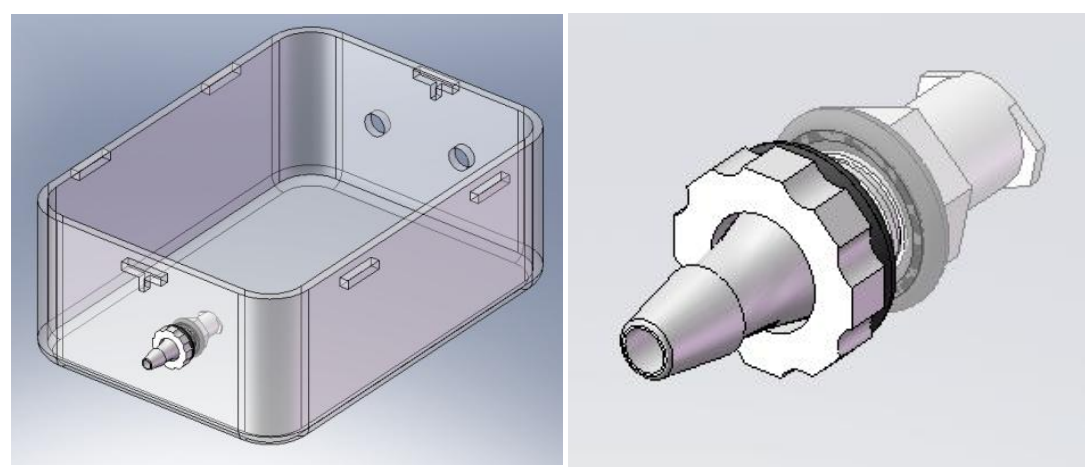
7. Once securely tightened, place a drop of Loctite 4011 adhesive (McMaster-Carr P/N 1818A4) between the lock nut and the threaded portion of the female luer lug panel mount.

8. Repeat steps 3-7 for assembly of the luminal outlet and extra luminal outlet holes.
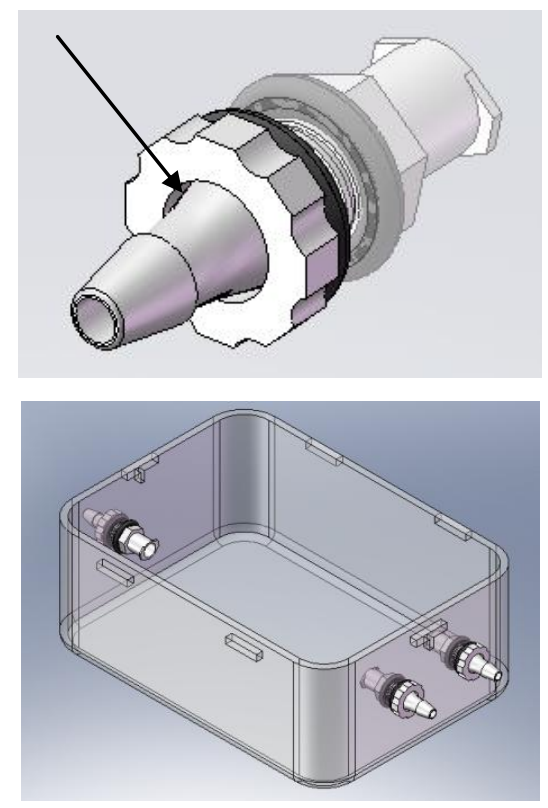

9. Attach a 2" piece of Tygon® silicone tubing, 1/8" ID (Cole-Parmer P/N WU-95702-06) to the barbed fitting of the luminal inlet.

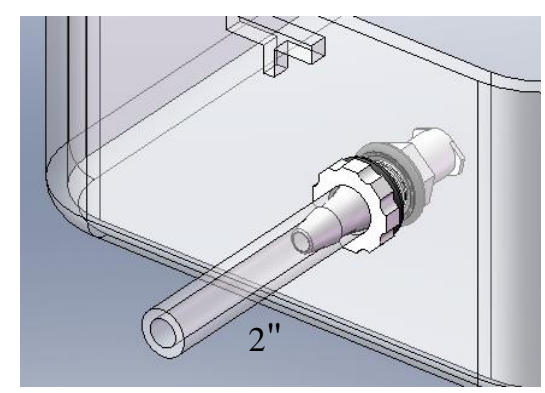

10. Attach a female luer thread-1/8" ID barb (Value Plastics P/N FTLL230-1) to the open end of the silicone tubing attached to the luminal inlet.

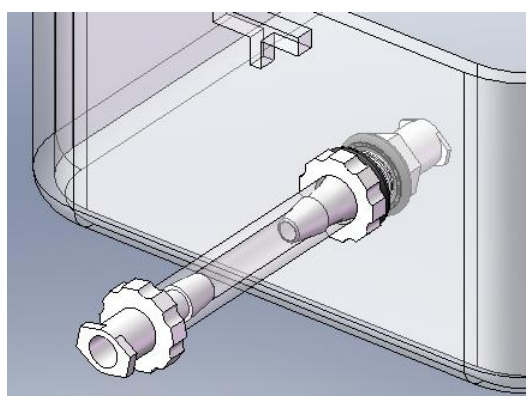

11. Secure the male luer lock end of a 3-way stopcock (Value Plastics P/N VPB1000079N) to the female luer thread attached to the silicone tubing.

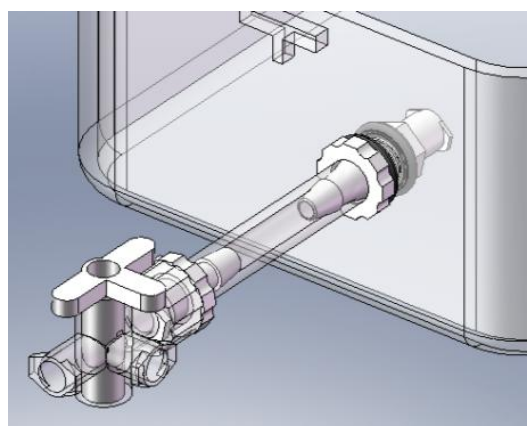


12. Attach a 5" piece of Tygon ${ }^{\circledR}$ silicone tubing, 1/8" ID (Cole-Parmer P/N WU95702-06) to the barbed fitting of the extra luminal outlet.

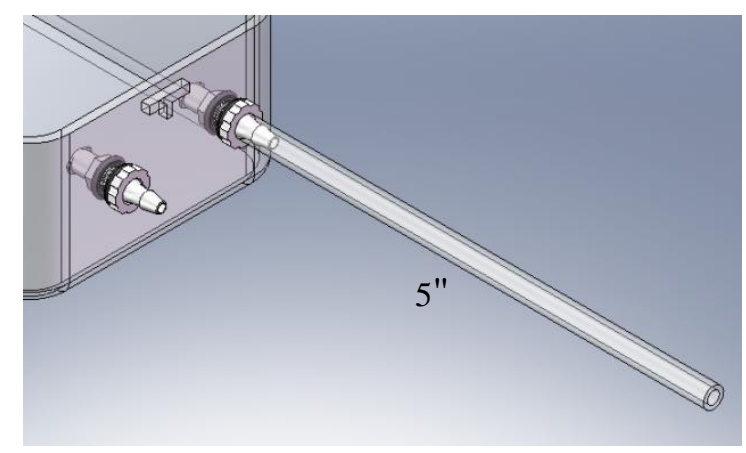

13. Attach a 1" piece of Tygon ${ }^{\circledR}$ silicone tubing, 1/8" ID (Cole-Parmer P/N WU-95702-06) to the barbed fitting of the luminal outlet.

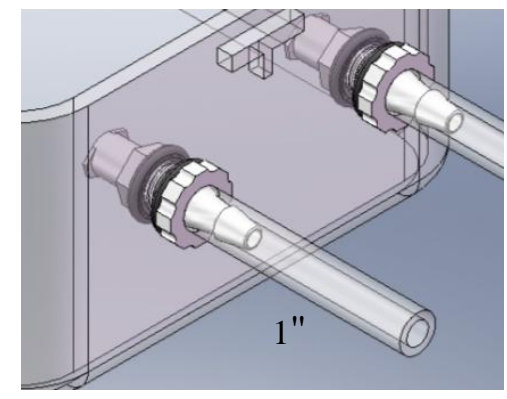

14. Attach a male luer lock-1/8" ID barb (Value Plastics P/N MTLL230-1) to the open end of the silicone tubing attached to the luminal outlet.

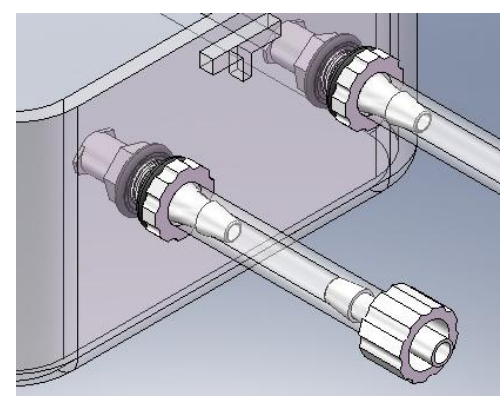

15. Secure the female luer thread end of a 1-way stopcock (Value Plastics P/N VP455980) to the male luer lock attached to the silicone tubing.

16. Attach a female luer thread-1/8" ID barb (Value Plastics P/N FTLL230-1) to the male luer lock end of the 1-way stopcock.
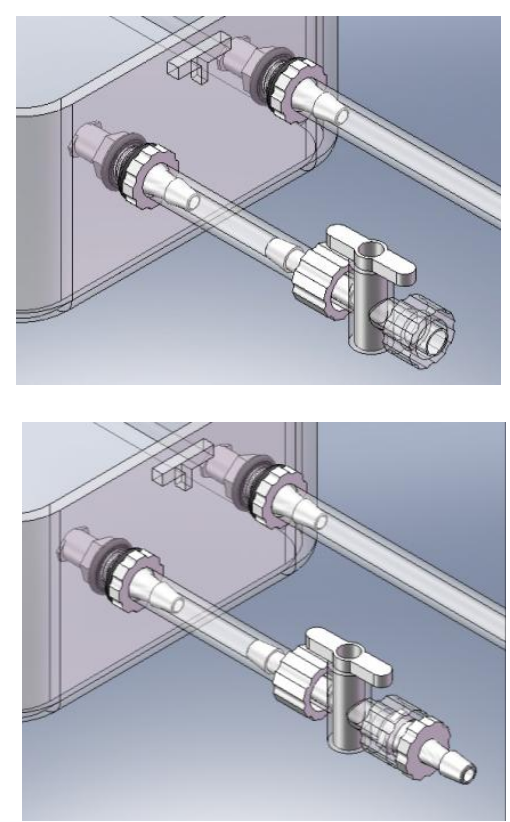
17. Attach a 2" piece of Tygon ${ }^{\circledR}$ silicone tubing, 1/8" ID (Cole-Parmer P/N WU-95702-06) to the barbed fitting of the female luer thread attached to the 1-way stopcock.

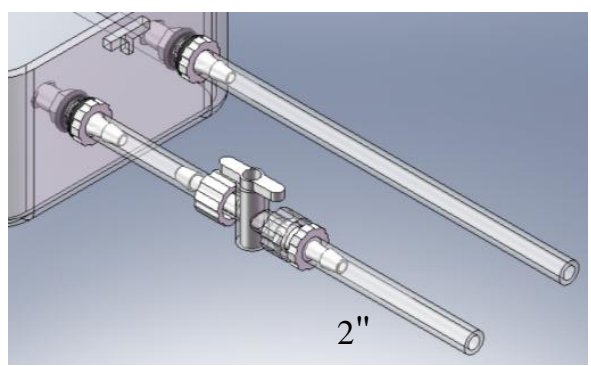

18. Insert the open ends of the silicone tubing attached to the luminal outlet and extra luminal outlet to a Y-connector with 1/8" ID barbs (Value Plastics P/N Y230-1).

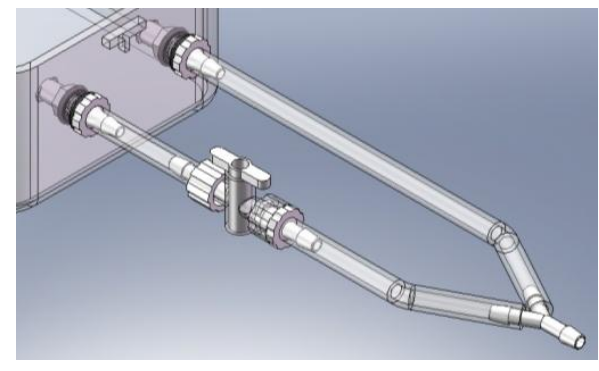

19. Attach a 1" piece of Tygon ${ }^{\circledR}$ silicone tubing, 1/8" ID (Cole-Parmer P/N WU-95702-06) to the barbed fitting of the Y-connector.

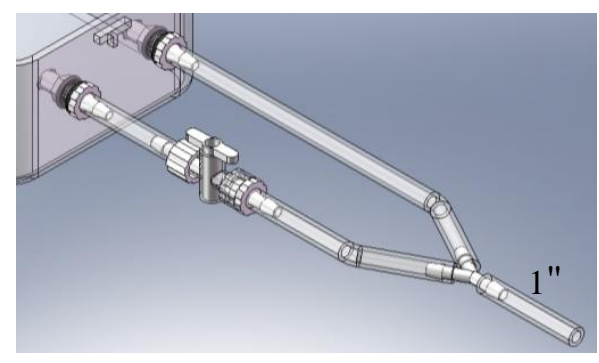

20. Attach a male luer lock-1/8" ID barb (Value Plastics P/N MTLL230-1) to the open end of the silicone tubing attached to the Y-connector.

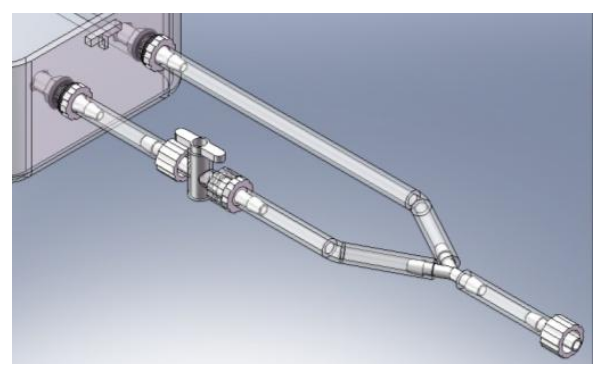

21. Secure the female luer thread end of a 1-way stopcock (Value Plastics P/N VP455980) to the male luer lock attached to the silicone tubing.

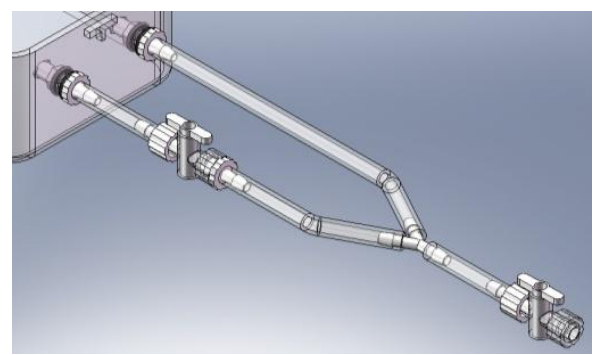


22. Place the lid with built in silicone o-ring on the assembled Ideal BVM chamber and close the four snapping latches.

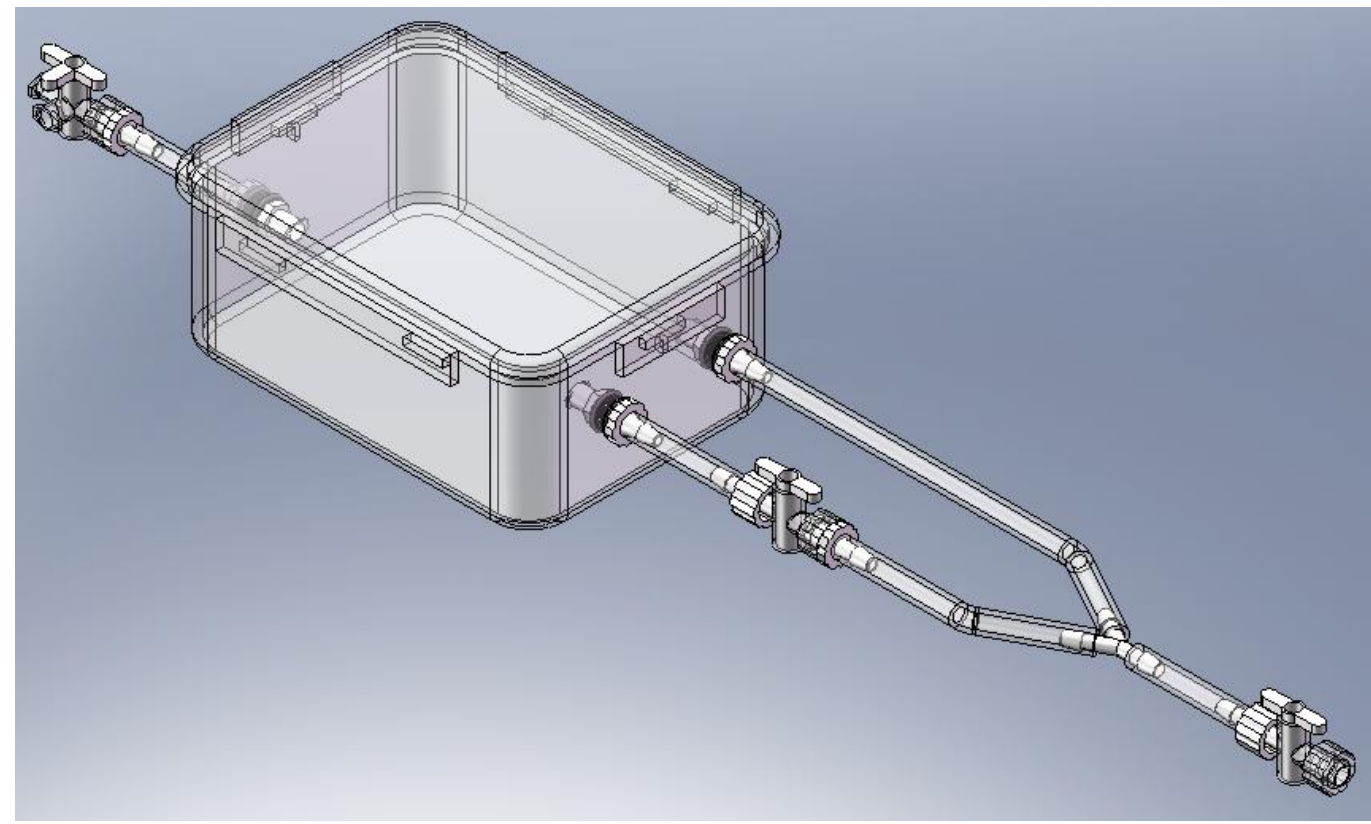


APPENDIX D: TWO-PORT MEDIA RESERVOIR ASSEMBLY

\begin{tabular}{|c|c|c|c|}
\hline Component & Company & Part Number & Quantity \\
\hline BD Falcon ${ }^{\text {TM }} 50 \mathrm{ml}$ Tube & BD Biosciences & 352098 & 1 EA \\
\hline Buna-N O-Ring & McMaster-Carr & $2418 T 112$ & 4 EA \\
\hline Loctite 4011 Adhesive & McMaster-Carr & 1818 A4 & 2 drops \\
\hline Female Luer Lug Panel Mount-1/8" Barb & Value Plastics & FTLB230-1 & 2 EA \\
\hline Lock Nut & Value Plastics & LNS-1 & 2 EA \\
\hline Male Luer Lock-1/8" Barb & Value Plastics & MTLL230-1 & 2 EA \\
\hline Female Luer Thread-1/8" Barb & Value Plastics & FTLL230-1 & 1 EA \\
\hline Tygon® Silicone Tubing, 1/8" ID & Cole-Parmer & WU-95702-06 & 21 inches \\
\hline
\end{tabular}

1. Drill two 15/64" holes into the lid of a BD Falcon ${ }^{\mathrm{TM}} 50$ ml tube (BD Biosciences P/N 352098).

2. Using a razor blade, remove any plastic debris that

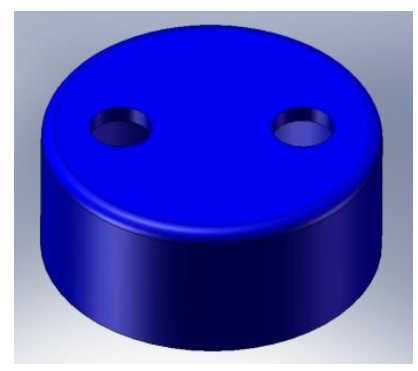
resulted from the use of the drill from the edges of the holes.

3. Slide a Buna-N o-ring (McMaster-Carr P/N 2418T112) onto a female luer lug panel mount-1/8" barb (Value Plastics P/N FTLB230-1).

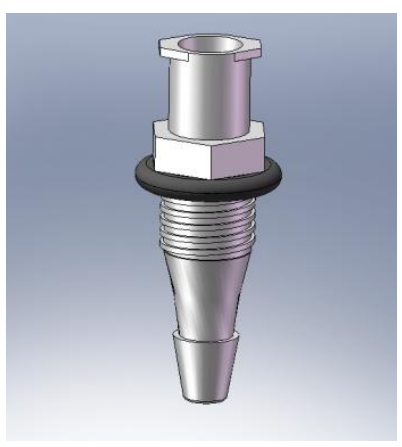


4. Thread the female luer lug panel mount with the Buna$\mathrm{N}$ o-ring through one of the drilled holes with the barbed portion exiting the top of the lid.

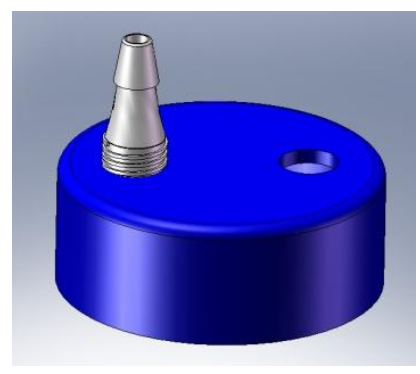

5. Slide a second Buna-N o-ring (McMaster-Carr P/N 2418T112) onto the barbed end of the female luer lug panel mount.

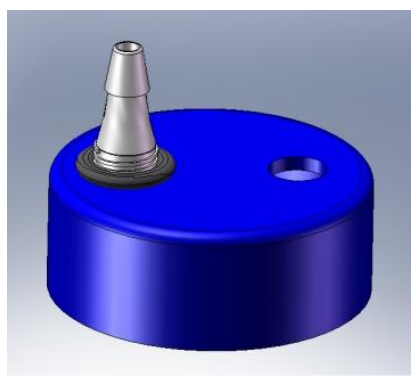

6. Tightly screw a lock nut (Value Plastics P/N LNS-1) onto the female luer lug panel mount, pressing the orings against the top and bottom of the lid.

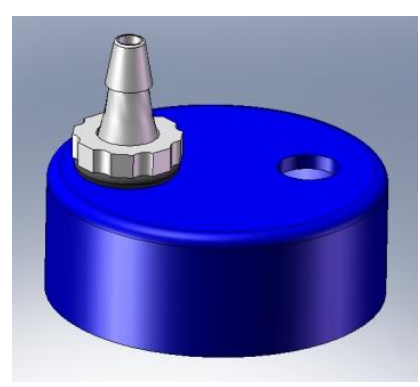

7. Once securely tightened, place a drop of Loctite 4011 adhesive (McMaster-Carr P/N 1818A4) between the lock nut and the threaded portion of the female luer lug panel mount.

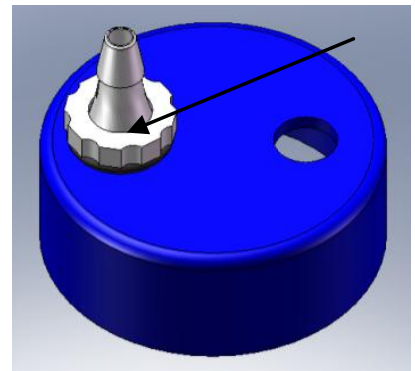

8. Repeat steps 3-7 for assembly of the second hole.

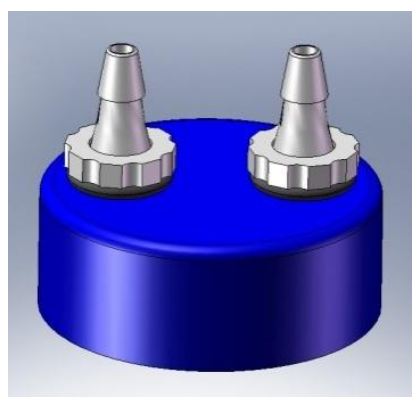


9. Attach a 1" piece of Tygon® silicone tubing, 1/8" ID (Cole-Parmer P/N WU-95702-06) to a male luer lock1/8" ID barb (Value Plastics P/N MTLL230-1).

10. Secure the male luer lock fitting of the silicone tubing assembly to one of the female luer lug panel mounts exiting the bottom of the lid.
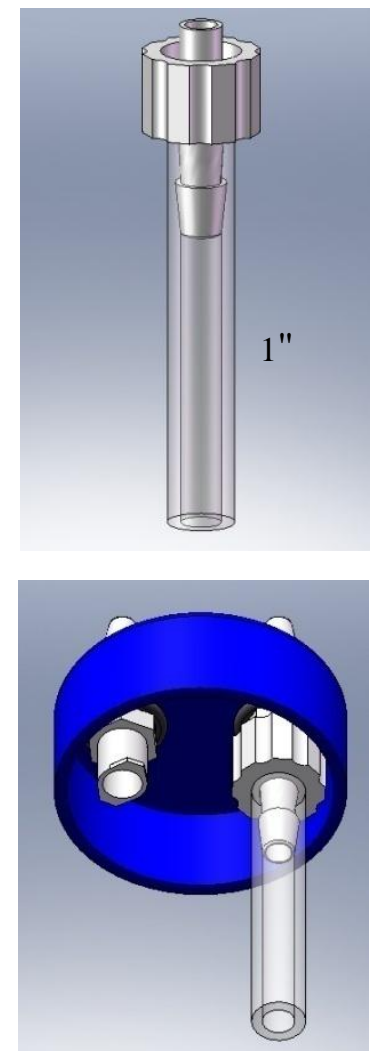

11. Attach two 10" pieces of Tygon ${ }^{\circledR}$ silicone tubing, 1/8" ID (Cole-Parmer P/N WU-95702-06) to the barbed ends of the female luer lug panel mounts exiting the top of the lid.

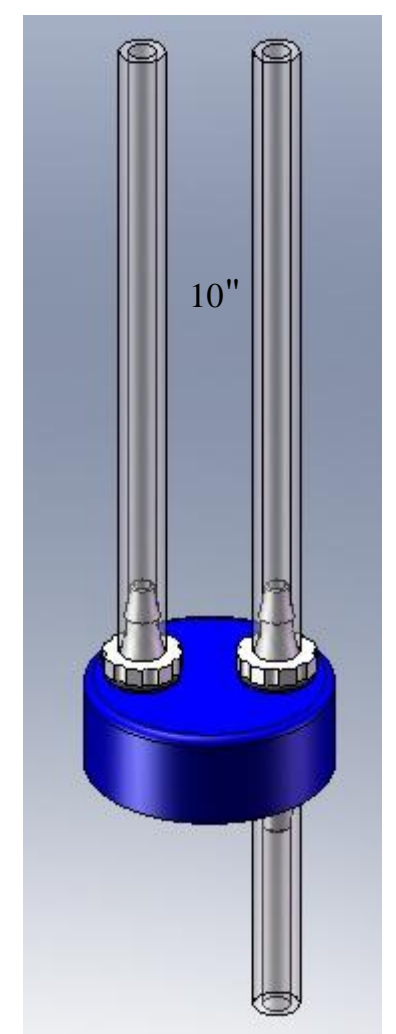


12. Attach a male luer lock-1/8" ID barb (Value Plastics P/N MTLL230-1) to the open end of the silicone tubing attached to the female luer lug panel mount with the $1 "$ silicone tubing assembly.

13. Attach a female luer thread-1/8" ID barb (Value Plastics P/N FTLL230-1) to the open end of the silicone tubing attached to the other female luer lug panel mount.
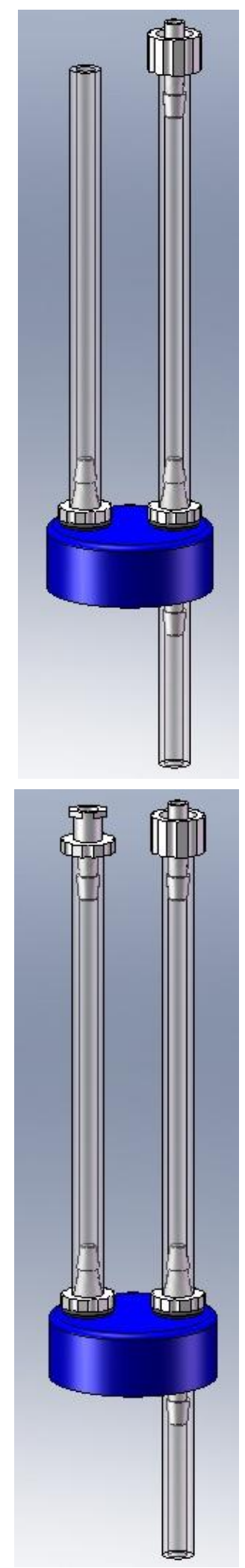
14. Screw the bottom section of the BD Falcon ${ }^{\mathrm{TM}} 50 \mathrm{ml}$ tube to the assembled twoport media reservoir lid.

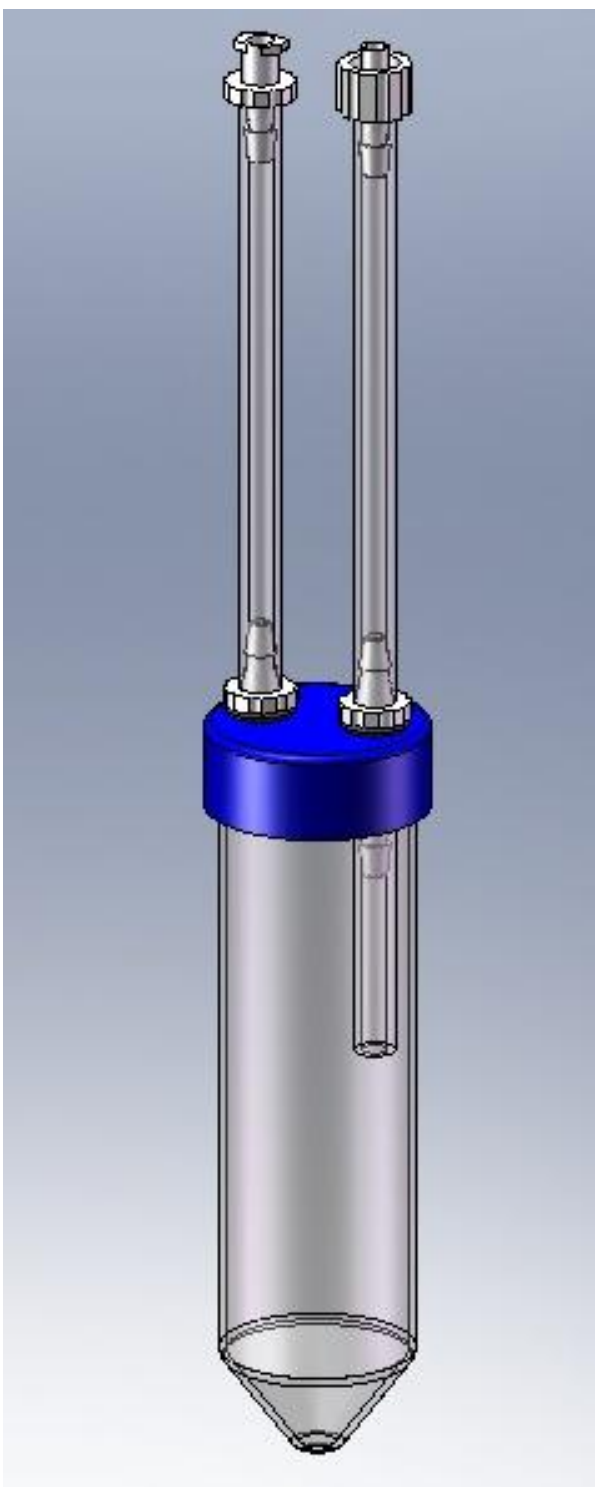


APPENDIX E: SCAFFOLD LENGTH VS NUMBER OF COUPLERS USED

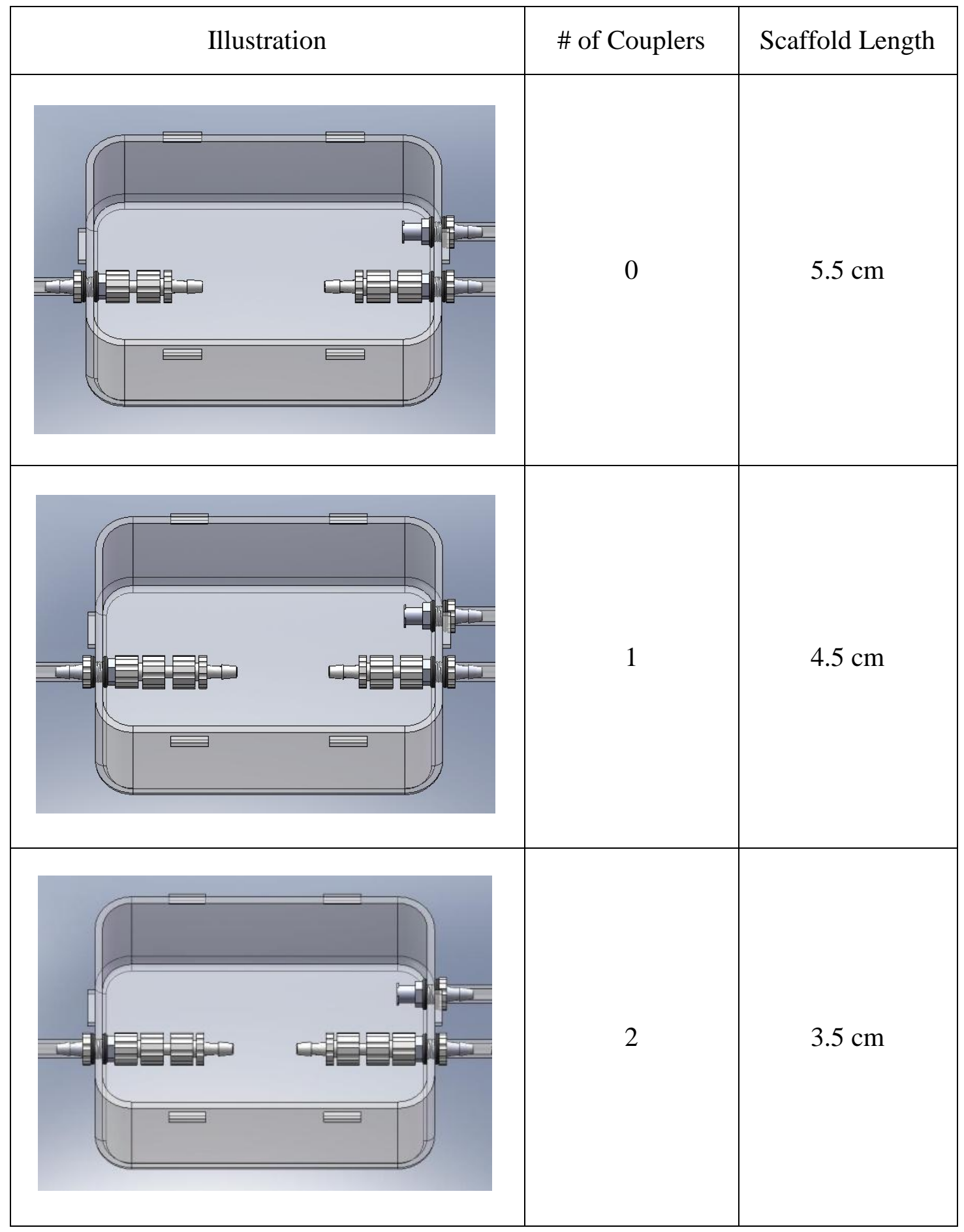




\section{APPENDIX F: BVM SYSTEM USER FEEDBACK SURVEY}

Name:

Date:

1. Overall Usability

Original BVM

Ideal BVM
Difficult

$\begin{array}{lllll}1 & 2 & 3 & 4 & 5\end{array}$

$\begin{array}{lllll}1 & 2 & 3 & 4 & 5\end{array}$

Easy

5

5

Comments:

2. Setup

Original BVM
Ideal BVM

Comments:

\begin{tabular}{rrrrl}
\multicolumn{2}{c}{ Difficult } & & \multicolumn{2}{c}{ Easy } \\
1 & 2 & 3 & 4 & 5 \\
1 & 2 & 3 & 4 & 5
\end{tabular}

Easy

\begin{tabular}{rrrrl}
\multicolumn{2}{r}{ Difficult } & & & Easy \\
1 & 2 & 3 & 4 & 5 \\
1 & 2 & 3 & 4 & 5
\end{tabular}

3. Scaffold Assembly

\begin{tabular}{lllll}
\multicolumn{2}{l}{ Difficult } & & & Easy \\
1 & 2 & 3 & 4 & 5 \\
1 & 2 & 3 & 4 & 5
\end{tabular}

Original BVM

Ideal BVM

Comments: 
4. Scaffold Insertion

Original BVM

Ideal BVM
Difficult

$\begin{array}{lllll}1 & 2 & 3 & 4 & 5\end{array}$

$\begin{array}{lllll}1 & 2 & 3 & 4 & 5\end{array}$

Comments:

5. Media Injection

Original BVM

Ideal BVM
Difficult

$\begin{array}{lllll}1 & 2 & 3 & 4 & 5\end{array}$

$\begin{array}{lllll}1 & 2 & 3 & 4 & 5\end{array}$

Easy

5

5

Comments:

6. Seal-ability

Original BVM

Ideal BVM
Difficult

1

1

Easy

5


7. Aseptic Use

Original BVM

Ideal BVM
Difficult

$\begin{array}{lllll}1 & 2 & 3 & 4 & 5\end{array}$

$\begin{array}{lllll}1 & 2 & 3 & 4 & 5\end{array}$

Comments:

8. Bioreactor Assembly

Original BVM

Ideal BVM
Difficult

$\begin{array}{lllll}1 & 2 & 3 & 4 & 5\end{array}$

$\begin{array}{lllll}1 & 2 & 3 & 4 & 5\end{array}$

Easy

5

5

Comments:

9. Control of Flow

Original BVM

Ideal BVM
Difficult

1

1

Easy

5


10. Adjustability

Original BVM

Ideal BVM
Difficult

$\begin{array}{lllll}1 & 2 & 3 & 4 & 5\end{array}$

$\begin{array}{lllll}1 & 2 & 3 & 4 & 5\end{array}$

Comments:

11. Components and Fittings

Original BVM

Ideal BVM
Awkward

1

12
Easy

5

5

Comments:

12. Component Configuration

Original BVM

Ideal BVM

\begin{tabular}{ccccc} 
Poor & & & \multicolumn{3}{r}{ Excellent } \\
1 & 2 & 3 & 4 & 5 \\
1 & 2 & 3 & 4 & 5
\end{tabular}

Comments: 


\section{BVM SYSTEM USER FEEDBACK SURVEY}

Name: Dimitri Delagrammaticas

Date: $07 / 01 / 08$

1. Overall Usability

Difficult

Easy

Original BVM

$\begin{array}{lllll}1 & 2 & 3 & 4 & 5\end{array}$

Ideal BVM

$\begin{array}{lllll}1 & 2 & 3 & 4 & 5\end{array}$

Comments:

still need a non-sterile partner but fittings and lid make overall use easier

2. Setup

Difficult

Easy

Original BVM

$\begin{array}{lllll}1 & 2 & 3 & 4 & 5\end{array}$

Ideal BVM

$\begin{array}{lllll}1 & 2 & 3 & 4 & 5\end{array}$

Comments:

separation of lid from chamber makes setup easier; male and female fittings ensure correct setup; no air bubbles; no tools needed

3. Scaffold Assembly

Difficult

Easy

Original BVM

$\begin{array}{lllll}1 & 2 & 3 & 4 & 5\end{array}$

Ideal BVM

12

4 5

\section{Comments:} no difference between old and new BVM; scaffold setup is easy 
4. Scaffold Insertion

Original BVM

Ideal BVM
Difficult

12

1

2

3 $4 \quad 5$

$\begin{array}{llll}2 & 3 & 4 & 5\end{array}$

Easy

5

5

\section{Comments:}

lid does not have to be held freeing up an extra hand for use; rotating luer lock fittings make insertion very easy

5. Media Injection

Difficult

Easy

Original BVM

1

2

Ideal BVM

12

34

4

5

5

\section{Comments:}

media injection is the same for both systems through the three-way stopcock; air bubbles are easier to eliminate from new chamber but new chamber requires more media

6. Seal-ability

\begin{tabular}{lllll}
\multicolumn{2}{c}{ Difficult } & & & Easy \\
1 & 2 & 3 & 4 & 5 \\
1 & 2 & 3 & 4 & 5
\end{tabular}

Original BVM

Ideal BVM

\section{Comments:}

built-in o-ring seal is nice; lid is easy to snap to chamber and creates a strong leak-proof seal; no tools needed when snapping on lid 
7. Aseptic Use

Original BVM

Ideal BVM
Difficult

12

1

2

2

2

Comments: separating the lid from the chamber improves aseptic use; lid can be sterilized individually

8. Bioreactor Assembly

Difficult

Easy

Original BVM

Ideal BVM

$\begin{array}{lllll}1 & 2 & 3 & 4 & 5 \\ 1 & 2 & 3 & 4 & 5\end{array}$

Comments: only one way to connect chamber to media reservoir; fast and easy but new chamber is larger

9. Control of Flow

Original BVM

Ideal BVM

\begin{tabular}{lllll}
\multicolumn{2}{c}{ Difficult } & & & Easy \\
1 & 2 & 3 & 4 & 5 \\
1 & 2 & 3 & 4 & 5
\end{tabular}

Comments:

stopcocks are easier to use than side clamps and firmly block flow; placement of one-way stopcocks makes control of flow easy 
10. Adjustability

Original BVM

Ideal BVM
Difficult

$\begin{array}{llll}1 & 2 & 3 & 4\end{array}$

12

Easy

5

5

\section{Comments:}

adjustability is limited to couplers; adjusting with couplers is easy and provides consistent scaffold lengths

11. Components and Fittings

Original BVM

Ideal BVM
Awkward

12

12

Convenient

4 5

45

\section{Comments:}

elimination of UV glue improves component setup; components and fittings are simple to use

12. Component Configuration

Original BVM

Ideal BVM
Poor

Excellent

1

12

1

\section{Comments:}

one-way connections to reservoir eliminates the confusion on which end to connect when assembling the complete bioreactor system; connection piece used in media reservoir sterilization is eliminated 


\section{APPENDIX G: REPRINT PERMISSIONS}

Rightslink Printable License

Page 1 of 5

\section{ELSEVIER LIMITED LICENSE TERMS AND CONDITIONS}

Jul 05, 2008

This is a License Agreement between Sara M Leifer ("You") and Elsevier Limited ("Elsevier Limited"). The license consists of your order details, the terms and conditions provided by Elsevier Limited, and the payment terms and conditions.

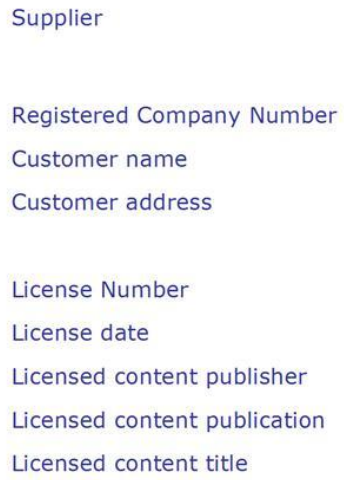

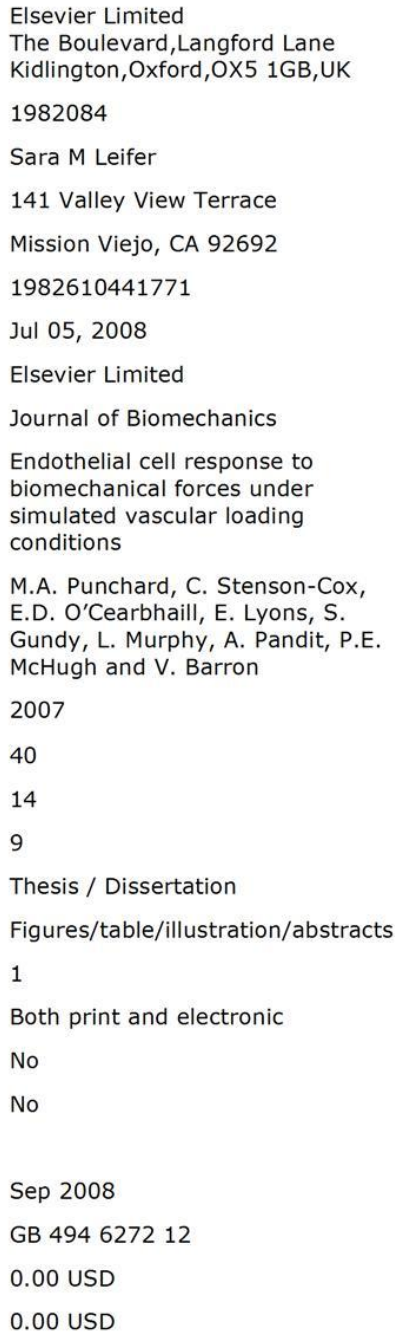


Terms and Conditions

\section{INTRODUCTION}

1. The publisher for this copyrighted material is Elsevier. By clicking "accept" in connection with completing this licensing transaction, you agree that the following terms and conditions apply to this transaction (along with the Billing and Payment terms and conditions established by Copyright Clearance Center, Inc. ("CCC"), at the time that you opened your Rightslink account and that are available at any time at $<\mathrm{http}: / /$ myaccount.copyright.com $>$ ).

\section{GENERAL TERMS}

2. Elsevier hereby grants you permission to reproduce the aforementioned material subject to the terms and conditions indicated.

3. Acknowledgement: If any part of the material to be used (for example, figures) has appeared in our publication with credit or acknowledgement to another source, permission must also be sought from that source. If such permission is not obtained then that material may not be included in your publication/copies. Suitable acknowledgement to the source must be made, either as a footnote or in a reference list at the end of your publication, as follows:

"Reprinted from Publication title, Vol /edition number, Author(s), Title of article / title of chapter, Pages No., Copyright (Year), with permission from Elsevier [OR APPLICABLE SOCIETY COPYRIGHT OWNER]." Also Lancet special credit - "Reprinted from The Lancet, Vol. number, Author(s), Title of article, Pages No., Copyright (Year), with permission from Elsevier."

4. Reproduction of this material is confined to the purpose and/or media for which permission is hereby given.

5. Altering/Modifying Material: Not Permitted. However figures and illustrations may be altered/adapted minimally to serve your work. Any other abbreviations, additions, deletions and/or any other alterations shall be made only with prior written authorization of Elsevier Ltd. (Please contact Elsevier at permissions@elsevier.com)

6. If the permission fee for the requested use of our material is waived in this instance, please be advised that your future requests for Elsevier materials may attract a fee.

7. Reservation of Rights: Publisher reserves all rights not specifically granted in the combination of (i) the license details provided by you and accepted in the course of this licensing transaction, (ii) these terms and conditions and (iii) CCC's Billing and Payment terms and conditions.

8. License Contingent Upon Payment: While you may exercise the rights licensed immediately upon issuance of the license at the end of the licensing process for the transaction, provided that you have disclosed complete and accurate details of your proposed 
use, no license is finally effective unless and until full payment is received from you (either by publisher or by CCC) as provided in CCC's Billing and Payment terms and conditions. If full payment is not received on a timely basis, then any license preliminarily granted shall be deemed automatically revoked and shall be void as if never granted. Further, in the event that you breach any of these terms and conditions or any of CCC's Billing and Payment terms and conditions, the license is automatically revoked and shall be void as if never granted. Use of materials as described in a revoked license, as well as any use of the materials beyond the scope of an unrevoked license, may constitute copyright infringement and publisher reserves the right to take any and all action to protect its copyright in the materials.

9. Warranties: Publisher makes no representations or warranties with respect to the licensed material.

10. Indemnity: You hereby indemnify and agree to hold harmless publisher and CCC, and their respective officers, directors, employees and agents, from and against any and all claims arising out of your use of the licensed material other than as specifically authorized pursuant to this license.

11. No Transfer of License: This license is personal to you and may not be sublicensed, assigned, or transferred by you to any other person without publisher's written permission.

12. No Amendment Except in Writing: This license may not be amended except in a writing signed by both parties (or, in the case of publisher, by CCC on publisher's behalf).

13. Objection to Contrary Terms: Publisher hereby objects to any terms contained in any purchase order, acknowledgment, check endorsement or other writing prepared by you, which terms are inconsistent with these terms and conditions or CCC's Billing and Payment terms and conditions. These terms and conditions, together with CCC's Billing and Payment terms and conditions (which are incorporated herein), comprise the entire agreement between you and publisher (and CCC) concerning this licensing transaction. In the event of any conflict between your obligations established by these terms and conditions and those established by CCC's Billing and Payment terms and conditions, these terms and conditions shall control.

14. Revocation: Elsevier or Copyright Clearance Center may deny the permissions described in this License at their sole discretion, for any reason or no reason, with a full refund payable to you. Notice of such denial will be made using the contact information provided by you. Failure to receive such notice will not alter or invalidate the denial. In no event will Elsevier or Copyright Clearance Center be responsible or liable for any costs, expenses or damage incurred by you as a result of a denial of your permission request, other than a refund of the amount(s) paid by you to Elsevier and/or Copyright Clearance Center for denied permissions.

\section{LIMITED LICENSE}

The following terms and conditions apply to specific license types:

15. Translation: This permission is granted for non-exclusive world English rights only unless your license was granted for translation rights. If you licensed translation rights you

https://s100.copyright.com/App/PrintableLicenseFrame.jsp?publisherID=70\&licenseID=20 $\ldots \quad 7 / 5 / 2008$ 
may only translate this content into the languages you requested. A professional translator must perform all translations and reproduce the content word for word preserving the integrity of the article. If this license is to re-use 1 or 2 figures then permission is granted for non-exclusive world rights in all languages.

16. Website: The following terms and conditions apply to electronic reserve and author websites:

Electronic reserve: If licensed material is to be posted to website, the web site is to be password-protected and made available only to bona fide students registered on a relevant course if:

This license was made in connection with a course,

This permission is granted for 1 year only. You may obtain a license for future website posting,

All content posted to the web site must maintain the copyright information line on the bottom of each image,

A hyper-text must be included to the Homepage of the journal from which you are licensing at http://www.sciencedirect.com/science/journal/xxxxx or the Elsevier homepage for books at http://www.elsevier.com, and

Central Storage: This license does not include permission for a scanned version of the material to be stored in a central repository such as that provided by Heron/XanEdu.

17. Author website for journals with the following additional clauses:

This permission is granted for 1 year only. You may obtain a license for future website posting,

All content posted to the web site must maintain the copyright information line on the bottom of each image, and

The permission granted is limited to the personal version of your paper. You are not allowed to download and post the published electronic version of your article (whether PDF or HTML, proof or final version), nor may you scan the printed edition to create an electronic version,

A hyper-text must be included to the Homepage of the journal from which you are licensing at http://www.sciencedirect.com/science/journal/xxxxx , or the Elsevier homepage for books at http://www.elsevier.com and

Central Storage: This license does not include permission for a scanned version of the material to be stored in a central repository such as that provided by Heron/XanEdu.

18. Author website for books with the following additional clauses:

Authors are permitted to place a brief summary of their work online only.

A hyper-text must be included to the Elsevier homepage at http://www.elsevier.com This permission is granted for 1 year only. You may obtain a license for future website posting,

All content posted to the web site must maintain the copyright information line on the bottom of each image, and

The permission granted is limited to the personal version of your paper. You are not allowed to download and post the published electronic version of your article (whether PDF or HTML, proof or final version), nor may you scan the printed edition to create an electronic version,

A hyper-text must be included to the Homepage of the journal from which you are licensing at http://www.sciencedirect.com/science/journal/xxxxx, or the Elsevier homepage for books at http://www.elsevier.com and 
Central Storage: This license does not include permission for a scanned version of the material to be stored in a central repository such as that provided by Heron/XanEdu.

19. Website (regular and for author): "A hyper-text must be included to the Homepage of the journal from which you are licensing at

http://www.sciencedirect.com/science/journal/xxxxx."

20. Thesis/Dissertation: If your license is for use in a thesis/dissertation your thesis may be submitted to your institution in either print or electronic form. Should your thesis be published commercially, please reapply for permission. These requirements include permission for the Library and Archives of Canada to supply single copies, on demand, of the complete thesis and include permission for UMI to supply single copies, on demand, of the complete thesis. Should your thesis be published commercially, please reapply for permission.

v1.2

\section{Other conditions:}

None

https://s100.copyright.com/App/PrintableLicenseFrame.jsp?publisherID=70\&licenseID=20 $\ldots \quad 7 / 5 / 2008$ 
ELSEVIER LIMITED LICENSE TERMS AND CONDITIONS

Jul 05, 2008

This is a License Agreement between Sara M Leifer ("You") and Elsevier Limited ("Elsevier Limited"). The license consists of your order details, the terms and conditions provided by Elsevier Limited, and the payment terms and conditions.

\begin{tabular}{|c|c|}
\hline Supplier & $\begin{array}{l}\text { Elsevier Limited } \\
\text { The Boulevard,Langford Lane } \\
\text { Kidlington,Oxford,OX5 1GB,UK }\end{array}$ \\
\hline Registered Company Number & 1982084 \\
\hline Customer name & Sara M Leifer \\
\hline \multirow[t]{2}{*}{ Customer address } & 141 Valley View Terrace \\
\hline & Mission Viejo, CA 92692 \\
\hline License Number & 1982630932917 \\
\hline License date & Jul 05, 2008 \\
\hline Licensed content publisher & Elsevier Limited \\
\hline Licensed content publication & Biomaterials \\
\hline Licensed content title & $\begin{array}{l}\text { Mechano-active tissue } \\
\text { engineering of vascular smooth } \\
\text { muscle using pulsatile perfusion } \\
\text { bioreactors and elastic PLCL } \\
\text { scaffolds }\end{array}$ \\
\hline Licensed content author & $\begin{array}{l}\text { Sung In Jeong, Jae Hyun Kwon, } \\
\text { Jin Ik Lim, Seung-Woo Cho, } \\
\text { Youngmee Jung, Won Jun Sung, } \\
\text { Soo Hyun Kim, Young Ha Kim, } \\
\text { Young Moo Lee, Byung-Soo Kim, } \\
\text { Cha Yong Choi and Soo-Ja Kim }\end{array}$ \\
\hline Licensed content date & April 2005 \\
\hline Volume number & 26 \\
\hline Issue number & 12 \\
\hline Pages & 7 \\
\hline Type of Use & Thesis / Dissertation \\
\hline Portion & Figures/table/illustration/abstracts \\
\hline Portion Quantity & 1 \\
\hline Format & Both print and electronic \\
\hline You are an author of the Elsevier article & No \\
\hline Are you translating? & No \\
\hline \multicolumn{2}{|l|}{ Purchase order number } \\
\hline Expected publication date & Sep 2008 \\
\hline Elsevier VAT number & GB 494627212 \\
\hline
\end{tabular}

https://s100.copyright.com/App/PrintableLicenseFrame.jsp?publisherID=70\&licenseID=20 $\ldots \quad 7 / 5 / 2008$ 
Permissions price

Value added $\operatorname{tax} 0.0 \%$

Total

Terms and Conditions
0.00 USD

0.00 USD

0.00 USD

\section{INTRODUCTION}

1. The publisher for this copyrighted material is Elsevier. By clicking "accept" in connection with completing this licensing transaction, you agree that the following terms and conditions apply to this transaction (along with the Billing and Payment terms and conditions established by Copyright Clearance Center, Inc. ("CCC"), at the time that you opened your Rightslink account and that are available at any time at $<\mathrm{http}: / /$ myaccount.copyright.com>).

\section{GENERAL TERMS}

2. Elsevier hereby grants you permission to reproduce the aforementioned material subject to the terms and conditions indicated.

3. Acknowledgement: If any part of the material to be used (for example, figures) has appeared in our publication with credit or acknowledgement to another source, permission must also be sought from that source. If such permission is not obtained then that material may not be included in your publication/copies. Suitable acknowledgement to the source must be made, either as a footnote or in a reference list at the end of your publication, as follows:

"Reprinted from Publication title, Vol /edition number, Author(s), Title of article / title of chapter, Pages No., Copyright (Year), with permission from Elsevier [OR APPLICABLE SOCIETY COPYRIGHT OWNER]." Also Lancet special credit - "Reprinted from The Lancet, Vol. number, Author(s), Title of article, Pages No., Copyright (Year), with permission from Elsevier."

4. Reproduction of this material is confined to the purpose and/or media for which permission is hereby given.

5. Altering/Modifying Material: Not Permitted. However figures and illustrations may be altered/adapted minimally to serve your work. Any other abbreviations, additions, deletions and/or any other alterations shall be made only with prior written authorization of Elsevier Ltd. (Please contact Elsevier at permissions@elsevier.com)

6. If the permission fee for the requested use of our material is waived in this instance, please be advised that your future requests for Elsevier materials may attract a fee.

7. Reservation of Rights: Publisher reserves all rights not specifically granted in the combination of (i) the license details provided by you and accepted in the course of this licensing transaction, (ii) these terms and conditions and (iii) CCC's Billing and Payment terms and conditions. 
8. License Contingent Upon Payment: While you may exercise the rights licensed immediately upon issuance of the license at the end of the licensing process for the transaction, provided that you have disclosed complete and accurate details of your proposed use, no license is finally effective unless and until full payment is received from you (either by publisher or by CCC) as provided in CCC's Billing and Payment terms and conditions. If full payment is not received on a timely basis, then any license preliminarily granted shall be deemed automatically revoked and shall be void as if never granted. Further, in the event that you breach any of these terms and conditions or any of CCC's Billing and Payment terms and conditions, the license is automatically revoked and shall be void as if never granted. Use of materials as described in a revoked license, as well as any use of the materials beyond the scope of an unrevoked license, may constitute copyright infringement and publisher reserves the right to take any and all action to protect its copyright in the materials.

9. Warranties: Publisher makes no representations or warranties with respect to the licensed material.

10. Indemnity: You hereby indemnify and agree to hold harmless publisher and CCC, and their respective officers, directors, employees and agents, from and against any and all claims arising out of your use of the licensed material other than as specifically authorized pursuant to this license.

11. No Transfer of License: This license is personal to you and may not be sublicensed, assigned, or transferred by you to any other person without publisher's written permission.

12. No Amendment Except in Writing: This license may not be amended except in a writing signed by both parties (or, in the case of publisher, by CCC on publisher's behalf).

13. Objection to Contrary Terms: Publisher hereby objects to any terms contained in any purchase order, acknowledgment, check endorsement or other writing prepared by you, which terms are inconsistent with these terms and conditions or CCC's Billing and Payment terms and conditions. These terms and conditions, together with CCC's Billing and Payment terms and conditions (which are incorporated herein), comprise the entire agreement between you and publisher (and CCC) concerning this licensing transaction. In the event of any conflict between your obligations established by these terms and conditions and those established by CCC's Billing and Payment terms and conditions, these terms and conditions shall control.

14. Revocation: Elsevier or Copyright Clearance Center may deny the permissions described in this License at their sole discretion, for any reason or no reason, with a full refund payable to you. Notice of such denial will be made using the contact information provided by you. Failure to receive such notice will not alter or invalidate the denial. In no event will Elsevier or Copyright Clearance Center be responsible or liable for any costs, expenses or damage incurred by you as a result of a denial of your permission request, other than a refund of the amount(s) paid by you to Elsevier and/or Copyright Clearance Center for denied permissions.

\section{LIMITED LICENSE}

The following terms and conditions apply to specific license types: 
15. Translation: This permission is granted for non-exclusive world English rights only unless your license was granted for translation rights. If you licensed translation rights you may only translate this content into the languages you requested. A professional translator must perform all translations and reproduce the content word for word preserving the integrity of the article. If this license is to re-use 1 or 2 figures then permission is granted for non-exclusive world rights in all languages.

16. Website: The following terms and conditions apply to electronic reserve and author websites:

Electronic reserve: If licensed material is to be posted to website, the web site is to be password-protected and made available only to bona fide students registered on a relevant course if:

This license was made in connection with a course,

This permission is granted for 1 year only. You may obtain a license for future website posting,

All content posted to the web site must maintain the copyright information line on the bottom of each image,

A hyper-text must be included to the Homepage of the journal from which you are licensing at http://www.sciencedirect.com/science/journal/xxxxx or the Elsevier homepage for books at http://www.elsevier.com , and

Central Storage: This license does not include permission for a scanned version of the material to be stored in a central repository such as that provided by Heron/XanEdu.

17. Author website for journals with the following additional clauses:

This permission is granted for 1 year only. You may obtain a license for future website posting,

All content posted to the web site must maintain the copyright information line on the bottom of each image, and

The permission granted is limited to the personal version of your paper. You are not allowed to download and post the published electronic version of your article (whether PDF or HTML, proof or final version), nor may you scan the printed edition to create an electronic version,

A hyper-text must be included to the Homepage of the journal from which you are licensing at http://www.sciencedirect.com/science/journal/xxxxx , or the Elsevier homepage for books at http://www.elsevier.com and

Central Storage: This license does not include permission for a scanned version of the material to be stored in a central repository such as that provided by Heron/XanEdu.

18. Author website for books with the following additional clauses:

Authors are permitted to place a brief summary of their work online only.

A hyper-text must be included to the Elsevier homepage at http://www.elsevier.com This permission is granted for 1 year only. You may obtain a license for future website posting,

All content posted to the web site must maintain the copyright information line on the bottom of each image, and

The permission granted is limited to the personal version of your paper. You are not allowed to download and post the published electronic version of your article (whether PDF or HTML, proof or final version), nor may you scan the printed edition to create an electronic version,

A hyper-text must be included to the Homepage of the journal from which you are licensing

https://s100.copyright.com/App/PrintableLicenseFrame.jsp?publisherID=70\&licenseID=20 $\ldots \quad 7 / 5 / 2008$ 
at http://www.sciencedirect.com/science/journal/xxxxx, or the Elsevier homepage for books at http://www.elsevier.com and

Central Storage: This license does not include permission for a scanned version of the material to be stored in a central repository such as that provided by Heron/XanEdu.

19. Website (regular and for author): "A hyper-text must be included to the Homepage of the journal from which you are licensing at

http://www.sciencedirect.com/science/journal/xxxxx."

20. Thesis/Dissertation: If your license is for use in a thesis/dissertation your thesis may be submitted to your institution in either print or electronic form. Should your thesis be published commercially, please reapply for permission. These requirements include permission for the Library and Archives of Canada to supply single copies, on demand, of the complete thesis and include permission for UMI to supply single copies, on demand, of the complete thesis. Should your thesis be published commercially, please reapply for permission.

v1.2

\section{Other conditions:}

None

https://s100.copyright.com/App/PrintableLicenseFrame.jsp?publisherID=70\&licenseID=20 $\ldots \quad 7 / 5 / 2008$ 
ELSEVIER LIMITED LICENSE TERMS AND CONDITIONS

Jul 05, 2008

This is a License Agreement between Sara M Leifer ("You") and Elsevier Limited ("Elsevier Limited"). The license consists of your order details, the terms and conditions provided by Elsevier Limited, and the payment terms and conditions.

\begin{tabular}{|c|c|}
\hline Supplier & $\begin{array}{l}\text { Elsevier Limited } \\
\text { The Boulevard,Langford Lane } \\
\text { Kidlington,Oxford,OX5 1GB,UK }\end{array}$ \\
\hline Registered Company Number & 1982084 \\
\hline Customer name & Sara M Leifer \\
\hline \multirow[t]{2}{*}{ Customer address } & 141 Valley View Terrace \\
\hline & Mission Viejo, CA 92692 \\
\hline License Number & 1982631211629 \\
\hline License date & Jul 05, 2008 \\
\hline Licensed content publisher & Elsevier Limited \\
\hline Licensed content publication & $\begin{array}{l}\text { European Journal of Cardio- } \\
\text { Thoracic Surgery }\end{array}$ \\
\hline Licensed content title & $\begin{array}{l}\text { Tissue engineering of small caliber } \\
\text { vascular grafts }\end{array}$ \\
\hline Licensed content author & $\begin{array}{l}\text { Simon P. Hoerstrup, Gregor Zünd, } \\
\text { Ralf Sodian, Andrea M. Schnell, } \\
\text { Jürg Grünenfelder and Marko I. } \\
\text { Turina }\end{array}$ \\
\hline Licensed content date & July 2001 \\
\hline Volume number & 20 \\
\hline Issue number & 1 \\
\hline Pages & 6 \\
\hline Type of Use & Thesis / Dissertation \\
\hline Portion & Figures/table/illustration/abstracts \\
\hline Portion Quantity & 1 \\
\hline Format & Both print and electronic \\
\hline You are an author of the Elsevier article & No \\
\hline Are you translating? & No \\
\hline \multicolumn{2}{|l|}{ Purchase order number } \\
\hline Expected publication date & Sep 2008 \\
\hline Elsevier VAT number & GB 494627212 \\
\hline Permissions price & 0.00 USD \\
\hline Value added tax $0.0 \%$ & 0.00 USD \\
\hline
\end{tabular}

https://s100.copyright.com/App/PrintableLicenseFrame.jsp?publisherID=70\&licenseID=20 $\ldots \quad 7 / 5 / 2008$ 
Terms and Conditions

\section{INTRODUCTION}

1. The publisher for this copyrighted material is Elsevier. By clicking "accept" in connection with completing this licensing transaction, you agree that the following terms and conditions apply to this transaction (along with the Billing and Payment terms and conditions established by Copyright Clearance Center, Inc. ("CCC"), at the time that you opened your Rightslink account and that are available at any time at $<\mathrm{http}: / /$ myaccount.copyright.com $>$ ).

\section{GENERAL TERMS}

2. Elsevier hereby grants you permission to reproduce the aforementioned material subject to the terms and conditions indicated.

3. Acknowledgement: If any part of the material to be used (for example, figures) has appeared in our publication with credit or acknowledgement to another source, permission must also be sought from that source. If such permission is not obtained then that material may not be included in your publication/copies. Suitable acknowledgement to the source must be made, either as a footnote or in a reference list at the end of your publication, as follows:

"Reprinted from Publication title, Vol /edition number, Author(s), Title of article / title of chapter, Pages No., Copyright (Year), with permission from Elsevier [OR APPLICABLE SOCIETY COPYRIGHT OWNER]." Also Lancet special credit - "Reprinted from The Lancet, Vol. number, Author(s), Title of article, Pages No., Copyright (Year), with permission from Elsevier."

4. Reproduction of this material is confined to the purpose and/or media for which permission is hereby given.

5. Altering/Modifying Material: Not Permitted. However figures and illustrations may be altered/adapted minimally to serve your work. Any other abbreviations, additions, deletions and/or any other alterations shall be made only with prior written authorization of Elsevier Ltd. (Please contact Elsevier at permissions@elsevier.com)

6. If the permission fee for the requested use of our material is waived in this instance, please be advised that your future requests for Elsevier materials may attract a fee.

7. Reservation of Rights: Publisher reserves all rights not specifically granted in the combination of (i) the license details provided by you and accepted in the course of this licensing transaction, (ii) these terms and conditions and (iii) CCC's Billing and Payment terms and conditions.

8. License Contingent Upon Payment: While you may exercise the rights licensed immediately upon issuance of the license at the end of the licensing process for the transaction, provided that you have disclosed complete and accurate details of your proposed 
use, no license is finally effective unless and until full payment is received from you (either by publisher or by CCC) as provided in CCC's Billing and Payment terms and conditions. If full payment is not received on a timely basis, then any license preliminarily granted shall be deemed automatically revoked and shall be void as if never granted. Further, in the event that you breach any of these terms and conditions or any of CCC's Billing and Payment terms and conditions, the license is automatically revoked and shall be void as if never granted. Use of materials as described in a revoked license, as well as any use of the materials beyond the scope of an unrevoked license, may constitute copyright infringement and publisher reserves the right to take any and all action to protect its copyright in the materials.

9. Warranties: Publisher makes no representations or warranties with respect to the licensed material.

10. Indemnity: You hereby indemnify and agree to hold harmless publisher and CCC, and their respective officers, directors, employees and agents, from and against any and all claims arising out of your use of the licensed material other than as specifically authorized pursuant to this license.

11. No Transfer of License: This license is personal to you and may not be sublicensed, assigned, or transferred by you to any other person without publisher's written permission.

12. No Amendment Except in Writing: This license may not be amended except in a writing signed by both parties (or, in the case of publisher, by CCC on publisher's behalf).

13. Objection to Contrary Terms: Publisher hereby objects to any terms contained in any purchase order, acknowledgment, check endorsement or other writing prepared by you, which terms are inconsistent with these terms and conditions or CCC's Billing and Payment terms and conditions. These terms and conditions, together with CCC's Billing and Payment terms and conditions (which are incorporated herein), comprise the entire agreement between you and publisher (and CCC) concerning this licensing transaction. In the event of any conflict between your obligations established by these terms and conditions and those established by CCC's Billing and Payment terms and conditions, these terms and conditions shall control.

14. Revocation: Elsevier or Copyright Clearance Center may deny the permissions described in this License at their sole discretion, for any reason or no reason, with a full refund payable to you. Notice of such denial will be made using the contact information provided by you. Failure to receive such notice will not alter or invalidate the denial. In no event will Elsevier or Copyright Clearance Center be responsible or liable for any costs, expenses or damage incurred by you as a result of a denial of your permission request, other than a refund of the amount(s) paid by you to Elsevier and/or Copyright Clearance Center for denied permissions.

\section{LIMITED LICENSE}

The following terms and conditions apply to specific license types:

15. Translation: This permission is granted for non-exclusive world English rights only unless your license was granted for translation rights. If you licensed translation rights you

https://s100.copyright.com/App/PrintableLicenseFrame.jsp?publisherID=70\&licenseID=20 $\ldots \quad 7 / 5 / 2008$ 
may only translate this content into the languages you requested. A professional translator must perform all translations and reproduce the content word for word preserving the integrity of the article. If this license is to re-use 1 or 2 figures then permission is granted for non-exclusive world rights in all languages.

16. Website: The following terms and conditions apply to electronic reserve and author websites:

Electronic reserve: If licensed material is to be posted to website, the web site is to be password-protected and made available only to bona fide students registered on a relevant course if:

This license was made in connection with a course,

This permission is granted for 1 year only. You may obtain a license for future website posting,

All content posted to the web site must maintain the copyright information line on the bottom of each image,

A hyper-text must be included to the Homepage of the journal from which you are licensing at http://www.sciencedirect.com/science/journal/xxxxx or the Elsevier homepage for books at http://www.elsevier.com, and

Central Storage: This license does not include permission for a scanned version of the material to be stored in a central repository such as that provided by Heron/XanEdu.

17. Author website for journals with the following additional clauses:

This permission is granted for 1 year only. You may obtain a license for future website posting,

All content posted to the web site must maintain the copyright information line on the bottom of each image, and

The permission granted is limited to the personal version of your paper. You are not allowed to download and post the published electronic version of your article (whether PDF or HTML, proof or final version), nor may you scan the printed edition to create an electronic version,

A hyper-text must be included to the Homepage of the journal from which you are licensing at http://www.sciencedirect.com/science/journal/xxxxx , or the Elsevier homepage for books at http://www.elsevier.com and

Central Storage: This license does not include permission for a scanned version of the material to be stored in a central repository such as that provided by Heron/XanEdu.

18. Author website for books with the following additional clauses:

Authors are permitted to place a brief summary of their work online only.

A hyper-text must be included to the Elsevier homepage at http://www.elsevier.com This permission is granted for 1 year only. You may obtain a license for future website posting,

All content posted to the web site must maintain the copyright information line on the bottom of each image, and

The permission granted is limited to the personal version of your paper. You are not allowed to download and post the published electronic version of your article (whether PDF or HTML, proof or final version), nor may you scan the printed edition to create an electronic version,

A hyper-text must be included to the Homepage of the journal from which you are licensing at http://www.sciencedirect.com/science/journal/xxxxx, or the Elsevier homepage for books at http://www.elsevier.com and 
Central Storage: This license does not include permission for a scanned version of the material to be stored in a central repository such as that provided by Heron/XanEdu.

19. Website (regular and for author): "A hyper-text must be included to the Homepage of the journal from which you are licensing at

http://www.sciencedirect.com/science/journal/xxxxx."

20. Thesis/Dissertation: If your license is for use in a thesis/dissertation your thesis may be submitted to your institution in either print or electronic form. Should your thesis be published commercially, please reapply for permission. These requirements include permission for the Library and Archives of Canada to supply single copies, on demand, of the complete thesis and include permission for UMI to supply single copies, on demand, of the complete thesis. Should your thesis be published commercially, please reapply for permission.

v1.2

\section{Other conditions:}

None

https://s100.copyright.com/App/PrintableLicenseFrame.jsp?publisherID=70\&licenseID=20 $\ldots \quad 7 / 5 / 2008$ 
ELSEVIER LIMITED LICENSE TERMS AND CONDITIONS

Jul 05, 2008

This is a License Agreement between Sara M Leifer ("You") and Elsevier Limited ("Elsevier Limited"). The license consists of your order details, the terms and conditions provided by Elsevier Limited, and the payment terms and conditions.

\begin{tabular}{|c|c|}
\hline Supplier & $\begin{array}{l}\text { Elsevier Limited } \\
\text { The Boulevard,Langford Lane } \\
\text { Kidlington,Oxford,OX5 1GB,UK }\end{array}$ \\
\hline Registered Company Number & 1982084 \\
\hline Customer name & Sara M Leifer \\
\hline \multirow[t]{2}{*}{ Customer address } & 141 Valley View Terrace \\
\hline & Mission Viejo, CA 92692 \\
\hline License Number & 1982631438829 \\
\hline License date & Jul 05, 2008 \\
\hline Licensed content publisher & Elsevier Limited \\
\hline Licensed content publication & Biomaterials \\
\hline Licensed content title & $\begin{array}{l}\text { Biological characterisation of } \\
\text { vascular grafts cultured in a } \\
\text { bioreactor }\end{array}$ \\
\hline Licensed content author & $\begin{array}{l}\text { Paula Engbers-Buijtenhuijs, Laura } \\
\text { Buttafoco, Andre A. Poot, Piet J. } \\
\text { Dijkstra, Rob A.I. de Vos, Lotus } \\
\text { M.Th. Sterk, Rob H. Geelkerken, } \\
\text { Istvan Vermes and Jan Feijen }\end{array}$ \\
\hline Licensed content date & April 2006 \\
\hline Volume number & 27 \\
\hline Issue number & 11 \\
\hline Pages & 8 \\
\hline Type of Use & Thesis / Dissertation \\
\hline Portion & Figures/table/illustration/abstracts \\
\hline Portion Quantity & 1 \\
\hline Format & Both print and electronic \\
\hline You are an author of the Elsevier article & No \\
\hline Are you translating? & No \\
\hline \multicolumn{2}{|l|}{ Purchase order number } \\
\hline Expected publication date & Sep 2008 \\
\hline Elsevier VAT number & GB 494627212 \\
\hline Permissions price & 0.00 USD \\
\hline Value added tax $0.0 \%$ & 0.00 USD \\
\hline
\end{tabular}

https://s100.copyright.com/App/PrintableLicenseFrame.jsp?publisherID=70\&licenseID=20 $\ldots \quad 7 / 5 / 2008$ 
Terms and Conditions

\section{INTRODUCTION}

1. The publisher for this copyrighted material is Elsevier. By clicking "accept" in connection with completing this licensing transaction, you agree that the following terms and conditions apply to this transaction (along with the Billing and Payment terms and conditions established by Copyright Clearance Center, Inc. ("CCC"), at the time that you opened your Rightslink account and that are available at any time at $<\mathrm{http}: / /$ myaccount.copyright.com $>$ ).

\section{GENERAL TERMS}

2. Elsevier hereby grants you permission to reproduce the aforementioned material subject to the terms and conditions indicated.

3. Acknowledgement: If any part of the material to be used (for example, figures) has appeared in our publication with credit or acknowledgement to another source, permission must also be sought from that source. If such permission is not obtained then that material may not be included in your publication/copies. Suitable acknowledgement to the source must be made, either as a footnote or in a reference list at the end of your publication, as follows:

"Reprinted from Publication title, Vol /edition number, Author(s), Title of article / title of chapter, Pages No., Copyright (Year), with permission from Elsevier [OR APPLICABLE SOCIETY COPYRIGHT OWNER]." Also Lancet special credit - "Reprinted from The Lancet, Vol. number, Author(s), Title of article, Pages No., Copyright (Year), with permission from Elsevier."

4. Reproduction of this material is confined to the purpose and/or media for which permission is hereby given.

5. Altering/Modifying Material: Not Permitted. However figures and illustrations may be altered/adapted minimally to serve your work. Any other abbreviations, additions, deletions and/or any other alterations shall be made only with prior written authorization of Elsevier Ltd. (Please contact Elsevier at permissions@elsevier.com)

6. If the permission fee for the requested use of our material is waived in this instance, please be advised that your future requests for Elsevier materials may attract a fee.

7. Reservation of Rights: Publisher reserves all rights not specifically granted in the combination of (i) the license details provided by you and accepted in the course of this licensing transaction, (ii) these terms and conditions and (iii) CCC's Billing and Payment terms and conditions.

8. License Contingent Upon Payment: While you may exercise the rights licensed immediately upon issuance of the license at the end of the licensing process for the transaction, provided that you have disclosed complete and accurate details of your proposed 
use, no license is finally effective unless and until full payment is received from you (either by publisher or by CCC) as provided in CCC's Billing and Payment terms and conditions. If full payment is not received on a timely basis, then any license preliminarily granted shall be deemed automatically revoked and shall be void as if never granted. Further, in the event that you breach any of these terms and conditions or any of CCC's Billing and Payment terms and conditions, the license is automatically revoked and shall be void as if never granted. Use of materials as described in a revoked license, as well as any use of the materials beyond the scope of an unrevoked license, may constitute copyright infringement and publisher reserves the right to take any and all action to protect its copyright in the materials.

9. Warranties: Publisher makes no representations or warranties with respect to the licensed material.

10. Indemnity: You hereby indemnify and agree to hold harmless publisher and CCC, and their respective officers, directors, employees and agents, from and against any and all claims arising out of your use of the licensed material other than as specifically authorized pursuant to this license.

11. No Transfer of License: This license is personal to you and may not be sublicensed, assigned, or transferred by you to any other person without publisher's written permission.

12. No Amendment Except in Writing: This license may not be amended except in a writing signed by both parties (or, in the case of publisher, by CCC on publisher's behalf).

13. Objection to Contrary Terms: Publisher hereby objects to any terms contained in any purchase order, acknowledgment, check endorsement or other writing prepared by you, which terms are inconsistent with these terms and conditions or CCC's Billing and Payment terms and conditions. These terms and conditions, together with CCC's Billing and Payment terms and conditions (which are incorporated herein), comprise the entire agreement between you and publisher (and CCC) concerning this licensing transaction. In the event of any conflict between your obligations established by these terms and conditions and those established by CCC's Billing and Payment terms and conditions, these terms and conditions shall control.

14. Revocation: Elsevier or Copyright Clearance Center may deny the permissions described in this License at their sole discretion, for any reason or no reason, with a full refund payable to you. Notice of such denial will be made using the contact information provided by you. Failure to receive such notice will not alter or invalidate the denial. In no event will Elsevier or Copyright Clearance Center be responsible or liable for any costs, expenses or damage incurred by you as a result of a denial of your permission request, other than a refund of the amount(s) paid by you to Elsevier and/or Copyright Clearance Center for denied permissions.

\section{LIMITED LICENSE}

The following terms and conditions apply to specific license types:

15. Translation: This permission is granted for non-exclusive world English rights only unless your license was granted for translation rights. If you licensed translation rights you

https://s100.copyright.com/App/PrintableLicenseFrame.jsp?publisherID=70\&licenseID=20 $\ldots \quad 7 / 5 / 2008$ 
may only translate this content into the languages you requested. A professional translator must perform all translations and reproduce the content word for word preserving the integrity of the article. If this license is to re-use 1 or 2 figures then permission is granted for non-exclusive world rights in all languages.

16. Website: The following terms and conditions apply to electronic reserve and author websites:

Electronic reserve: If licensed material is to be posted to website, the web site is to be password-protected and made available only to bona fide students registered on a relevant course if:

This license was made in connection with a course,

This permission is granted for 1 year only. You may obtain a license for future website posting,

All content posted to the web site must maintain the copyright information line on the bottom of each image,

A hyper-text must be included to the Homepage of the journal from which you are licensing at http://www.sciencedirect.com/science/journal/xxxxx or the Elsevier homepage for books at http://www.elsevier.com, and

Central Storage: This license does not include permission for a scanned version of the material to be stored in a central repository such as that provided by Heron/XanEdu.

17. Author website for journals with the following additional clauses:

This permission is granted for 1 year only. You may obtain a license for future website posting,

All content posted to the web site must maintain the copyright information line on the bottom of each image, and

The permission granted is limited to the personal version of your paper. You are not allowed to download and post the published electronic version of your article (whether PDF or HTML, proof or final version), nor may you scan the printed edition to create an electronic version,

A hyper-text must be included to the Homepage of the journal from which you are licensing at http://www.sciencedirect.com/science/journal/xxxxx , or the Elsevier homepage for books at http://www.elsevier.com and

Central Storage: This license does not include permission for a scanned version of the material to be stored in a central repository such as that provided by Heron/XanEdu.

18. Author website for books with the following additional clauses:

Authors are permitted to place a brief summary of their work online only.

A hyper-text must be included to the Elsevier homepage at http://www.elsevier.com This permission is granted for 1 year only. You may obtain a license for future website posting,

All content posted to the web site must maintain the copyright information line on the bottom of each image, and

The permission granted is limited to the personal version of your paper. You are not allowed to download and post the published electronic version of your article (whether PDF or HTML, proof or final version), nor may you scan the printed edition to create an electronic version,

A hyper-text must be included to the Homepage of the journal from which you are licensing at http://www.sciencedirect.com/science/journal/xxxxx, or the Elsevier homepage for books at http://www.elsevier.com and 
Central Storage: This license does not include permission for a scanned version of the material to be stored in a central repository such as that provided by Heron/XanEdu.

19. Website (regular and for author): "A hyper-text must be included to the Homepage of the journal from which you are licensing at

http://www.sciencedirect.com/science/journal/xxxxx."

20. Thesis/Dissertation: If your license is for use in a thesis/dissertation your thesis may be submitted to your institution in either print or electronic form. Should your thesis be published commercially, please reapply for permission. These requirements include permission for the Library and Archives of Canada to supply single copies, on demand, of the complete thesis and include permission for UMI to supply single copies, on demand, of the complete thesis. Should your thesis be published commercially, please reapply for permission.

v1.2

\section{Other conditions:}

None

https://s100.copyright.com/App/PrintableLicenseFrame.jsp?publisherID=70\&licenseID=20 $\ldots \quad 7 / 5 / 2008$ 
ELSEVIER LIMITED LICENSE TERMS AND CONDITIONS

Jul 19, 2008

This is a License Agreement between Sara M Leifer ("You") and Elsevier Limited ("Elsevier Limited"). The license consists of your order details, the terms and conditions provided by Elsevier Limited, and the payment terms and conditions.

\begin{tabular}{|c|c|}
\hline Supplier & $\begin{array}{l}\text { Elsevier Limited } \\
\text { The Boulevard,Langford Lane } \\
\text { Kidlington,Oxford,OX5 1GB,UK }\end{array}$ \\
\hline Registered Company Number & 1982084 \\
\hline Customer name & Sara M Leifer \\
\hline \multirow[t]{2}{*}{ Customer address } & 141 Valley View Terrace \\
\hline & Mission Viejo, CA 92692 \\
\hline License Number & 1992631289631 \\
\hline License date & Jul 19, 2008 \\
\hline Licensed content publisher & Elsevier Limited \\
\hline Licensed content publication & $\begin{array}{l}\text { Computer Methods and Programs } \\
\text { in Biomedicine }\end{array}$ \\
\hline Licensed content title & $\begin{array}{l}\text { An improved algorithm for vessel } \\
\text { centerline tracking in coronary } \\
\text { angiograms }\end{array}$ \\
\hline Licensed content author & $\begin{array}{l}\text { Yan Xu, Hui Zhang, Hao Li and } \\
\text { Guangshu Hu }\end{array}$ \\
\hline Licensed content date & November 2007 \\
\hline Volume number & 88 \\
\hline Issue number & 2 \\
\hline Pages & 13 \\
\hline Type of Use & Thesis / Dissertation \\
\hline Portion & Figures/table/illustration/abstracts \\
\hline Portion Quantity & 1 \\
\hline Format & Both print and electronic \\
\hline You are an author of the Elsevier article & No \\
\hline Are you translating? & No \\
\hline \multicolumn{2}{|l|}{ Purchase order number } \\
\hline Expected publication date & Sep 2008 \\
\hline Elsevier VAT number & GB 494627212 \\
\hline Permissions price & 0.00 USD \\
\hline Value added tax $0.0 \%$ & 0.00 USD \\
\hline
\end{tabular}

https://s100.copyright.com/App/PrintableLicenseFrame.jsp?publisherID=70\&licenseID=2 $\ldots \quad$ 7/19/2008 
Terms and Conditions

\section{INTRODUCTION}

1. The publisher for this copyrighted material is Elsevier. By clicking "accept" in connection with completing this licensing transaction, you agree that the following terms and conditions apply to this transaction (along with the Billing and Payment terms and conditions established by Copyright Clearance Center, Inc. ("CCC"), at the time that you opened your Rightslink account and that are available at any time at $<\mathrm{http}: / /$ myaccount.copyright.com $>$ ).

\section{GENERAL TERMS}

2. Elsevier hereby grants you permission to reproduce the aforementioned material subject to the terms and conditions indicated.

3. Acknowledgement: If any part of the material to be used (for example, figures) has appeared in our publication with credit or acknowledgement to another source, permission must also be sought from that source. If such permission is not obtained then that material may not be included in your publication/copies. Suitable acknowledgement to the source must be made, either as a footnote or in a reference list at the end of your publication, as follows:

"Reprinted from Publication title, Vol /edition number, Author(s), Title of article / title of chapter, Pages No., Copyright (Year), with permission from Elsevier [OR APPLICABLE SOCIETY COPYRIGHT OWNER]." Also Lancet special credit - "Reprinted from The Lancet, Vol. number, Author(s), Title of article, Pages No., Copyright (Year), with permission from Elsevier."

4. Reproduction of this material is confined to the purpose and/or media for which permission is hereby given.

5. Altering/Modifying Material: Not Permitted. However figures and illustrations may be altered/adapted minimally to serve your work. Any other abbreviations, additions, deletions and/or any other alterations shall be made only with prior written authorization of Elsevier Ltd. (Please contact Elsevier at permissions@elsevier.com)

6. If the permission fee for the requested use of our material is waived in this instance, please be advised that your future requests for Elsevier materials may attract a fee.

7. Reservation of Rights: Publisher reserves all rights not specifically granted in the combination of (i) the license details provided by you and accepted in the course of this licensing transaction, (ii) these terms and conditions and (iii) CCC's Billing and Payment terms and conditions.

8. License Contingent Upon Payment: While you may exercise the rights licensed immediately upon issuance of the license at the end of the licensing process for the transaction, provided that you have disclosed complete and accurate details of your proposed use, no license is finally effective unless and until full payment is received from you (either 
by publisher or by $\mathrm{CCC}$ ) as provided in CCC's Billing and Payment terms and conditions. If full payment is not received on a timely basis, then any license preliminarily granted shall be deemed automatically revoked and shall be void as if never granted. Further, in the event that you breach any of these terms and conditions or any of CCC's Billing and Payment terms and conditions, the license is automatically revoked and shall be void as if never granted. Use of materials as described in a revoked license, as well as any use of the materials beyond the scope of an unrevoked license, may constitute copyright infringement and publisher reserves the right to take any and all action to protect its copyright in the materials.

9. Warranties: Publisher makes no representations or warranties with respect to the licensed material.

10. Indemnity: You hereby indemnify and agree to hold harmless publisher and CCC, and their respective officers, directors, employees and agents, from and against any and all claims arising out of your use of the licensed material other than as specifically authorized pursuant to this license.

11. No Transfer of License: This license is personal to you and may not be sublicensed, assigned, or transferred by you to any other person without publisher's written permission.

12. No Amendment Except in Writing: This license may not be amended except in a writing signed by both parties (or, in the case of publisher, by CCC on publisher's behalf).

13. Objection to Contrary Terms: Publisher hereby objects to any terms contained in any purchase order, acknowledgment, check endorsement or other writing prepared by you, which terms are inconsistent with these terms and conditions or CCC's Billing and Payment terms and conditions. These terms and conditions, together with CCC's Billing and Payment terms and conditions (which are incorporated herein), comprise the entire agreement between you and publisher (and CCC) concerning this licensing transaction. In the event of any conflict between your obligations established by these terms and conditions and those established by CCC's Billing and Payment terms and conditions, these terms and conditions shall control.

14. Revocation: Elsevier or Copyright Clearance Center may deny the permissions described in this License at their sole discretion, for any reason or no reason, with a full refund payable to you. Notice of such denial will be made using the contact information provided by you. Failure to receive such notice will not alter or invalidate the denial. In no event will Elsevier or Copyright Clearance Center be responsible or liable for any costs, expenses or damage incurred by you as a result of a denial of your permission request, other than a refund of the amount(s) paid by you to Elsevier and/or Copyright Clearance Center for denied permissions.

\section{LIMITED LICENSE}

The following terms and conditions apply to specific license types:

15. Translation: This permission is granted for non-exclusive world English rights only unless your license was granted for translation rights. If you licensed translation rights you may only translate this content into the languages you requested. A professional translator 
must perform all translations and reproduce the content word for word preserving the integrity of the article. If this license is to re-use 1 or 2 figures then permission is granted for non-exclusive world rights in all languages.

16. Website: The following terms and conditions apply to electronic reserve and author websites:

Electronic reserve: If licensed material is to be posted to website, the web site is to be password-protected and made available only to bona fide students registered on a relevant course if:

This license was made in connection with a course,

This permission is granted for 1 year only. You may obtain a license for future website posting,

All content posted to the web site must maintain the copyright information line on the bottom of each image,

A hyper-text must be included to the Homepage of the journal from which you are licensing at http://www.sciencedirect.com/science/journal/xxxxx or the Elsevier homepage for books at http://www.elsevier.com , and

Central Storage: This license does not include permission for a scanned version of the material to be stored in a central repository such as that provided by Heron/XanEdu.

17. Author website for journals with the following additional clauses:

This permission is granted for 1 year only. You may obtain a license for future website posting,

All content posted to the web site must maintain the copyright information line on the bottom of each image, and

The permission granted is limited to the personal version of your paper. You are not allowed to download and post the published electronic version of your article (whether PDF or HTML, proof or final version), nor may you scan the printed edition to create an electronic version,

A hyper-text must be included to the Homepage of the journal from which you are licensing at http://www.sciencedirect.com/science/journal/xxxxx, or the Elsevier homepage for books at http://www.elsevier.com and

Central Storage: This license does not include permission for a scanned version of the material to be stored in a central repository such as that provided by Heron/XanEdu.

18. Author website for books with the following additional clauses:

Authors are permitted to place a brief summary of their work online only.

A hyper-text must be included to the Elsevier homepage at http://www.elsevier.com This permission is granted for 1 year only. You may obtain a license for future website posting,

All content posted to the web site must maintain the copyright information line on the bottom of each image, and

The permission granted is limited to the personal version of your paper. You are not allowed to download and post the published electronic version of your article (whether PDF or HTML, proof or final version), nor may you scan the printed edition to create an electronic version,

A hyper-text must be included to the Homepage of the journal from which you are licensing at http://www.sciencedirect.com/science/journal/xxxxx , or the Elsevier homepage for books at http://www.elsevier.com and

Central Storage: This license does not include permission for a scanned version of the 
material to be stored in a central repository such as that provided by Heron/XanEdu.

19. Website (regular and for author): "A hyper-text must be included to the Homepage of the journal from which you are licensing at

http://www.sciencedirect.com/science/journal/xxxxx."

20. Thesis/Dissertation: If your license is for use in a thesis/dissertation your thesis may be submitted to your institution in either print or electronic form. Should your thesis be published commercially, please reapply for permission. These requirements include permission for the Library and Archives of Canada to supply single copies, on demand, of the complete thesis and include permission for UMI to supply single copies, on demand, of the complete thesis. Should your thesis be published commercially, please reapply for permission.

$\mathrm{v} 1.2$

21. Other conditions:

None

https://s100.copyright.com/App/PrintableLicenseFrame.jsp?publisherID=70\&licenseID=2 $\ldots \quad$ 7/19/2008 
ELSEVIER LIMITED LICENSE TERMS AND CONDITIONS

This is a License Agreement between Sara M Leifer ("You") and Elsevier Limited ("Elsevier Limited"). The license consists of your order details, the terms and conditions provided by Elsevier Limited, and the payment terms and conditions.

\begin{tabular}{|c|c|}
\hline Supplier & $\begin{array}{l}\text { Elsevier Limited } \\
\text { The Boulevard,Langford Lane } \\
\text { Kidlington,Oxford,OX5 1GB,UK }\end{array}$ \\
\hline Registered Company Number & 1982084 \\
\hline Customer name & Sara M Leifer \\
\hline \multirow[t]{2}{*}{ Customer address } & 141 Valley View Terrace \\
\hline & Mission Viejo, CA 92692 \\
\hline License Number & 1992651301513 \\
\hline License date & Jul 19, 2008 \\
\hline Licensed content publisher & Elsevier Limited \\
\hline Licensed content publication & $\begin{array}{l}\text { Cardiovascular Revascularization } \\
\text { Medicine }\end{array}$ \\
\hline Licensed content title & $\begin{array}{l}\text { Novel stent and delivery systems } \\
\text { for the treatment of bifurcation } \\
\text { lesions: porcine coronary artery } \\
\text { model }\end{array}$ \\
\hline Licensed content author & $\begin{array}{l}\text { Fumiaki Ikeno, Maurice } \\
\text { Buchbinder and Alan C. Yeung }\end{array}$ \\
\hline Licensed content date & January-March 2007 \\
\hline Volume number & 8 \\
\hline Issue number & 1 \\
\hline Pages & 5 \\
\hline Type of Use & Thesis / Dissertation \\
\hline Portion & Figures/table/illustration/abstracts \\
\hline Portion Quantity & 2 \\
\hline Format & Both print and electronic \\
\hline You are an author of the Elsevier article & No \\
\hline Are you translating? & No \\
\hline \multicolumn{2}{|l|}{ Purchase order number } \\
\hline Expected publication date & Sep 2008 \\
\hline Elsevier VAT number & GB 494627212 \\
\hline Permissions price & 0.00 USD \\
\hline Value added tax $0.0 \%$ & 0.00 USD \\
\hline
\end{tabular}

https://s100.copyright.com/App/PrintableLicenseFrame.jsp?publisherID=70\&licenseID=2 $\ldots \quad$ 7/19/2008 
Terms and Conditions

\section{INTRODUCTION}

1. The publisher for this copyrighted material is Elsevier. By clicking "accept" in connection with completing this licensing transaction, you agree that the following terms and conditions apply to this transaction (along with the Billing and Payment terms and conditions established by Copyright Clearance Center, Inc. ("CCC"), at the time that you opened your Rightslink account and that are available at any time at $<\mathrm{http}: / /$ myaccount.copyright.com $>$ ).

\section{GENERAL TERMS}

2. Elsevier hereby grants you permission to reproduce the aforementioned material subject to the terms and conditions indicated.

3. Acknowledgement: If any part of the material to be used (for example, figures) has appeared in our publication with credit or acknowledgement to another source, permission must also be sought from that source. If such permission is not obtained then that material may not be included in your publication/copies. Suitable acknowledgement to the source must be made, either as a footnote or in a reference list at the end of your publication, as follows:

"Reprinted from Publication title, Vol /edition number, Author(s), Title of article / title of chapter, Pages No., Copyright (Year), with permission from Elsevier [OR APPLICABLE SOCIETY COPYRIGHT OWNER]." Also Lancet special credit - "Reprinted from The Lancet, Vol. number, Author(s), Title of article, Pages No., Copyright (Year), with permission from Elsevier."

4. Reproduction of this material is confined to the purpose and/or media for which permission is hereby given.

5. Altering/Modifying Material: Not Permitted. However figures and illustrations may be altered/adapted minimally to serve your work. Any other abbreviations, additions, deletions and/or any other alterations shall be made only with prior written authorization of Elsevier Ltd. (Please contact Elsevier at permissions@elsevier.com)

6. If the permission fee for the requested use of our material is waived in this instance, please be advised that your future requests for Elsevier materials may attract a fee.

7. Reservation of Rights: Publisher reserves all rights not specifically granted in the combination of (i) the license details provided by you and accepted in the course of this licensing transaction, (ii) these terms and conditions and (iii) CCC's Billing and Payment terms and conditions.

8. License Contingent Upon Payment: While you may exercise the rights licensed immediately upon issuance of the license at the end of the licensing process for the transaction, provided that you have disclosed complete and accurate details of your proposed 
use, no license is finally effective unless and until full payment is received from you (either by publisher or by CCC) as provided in CCC's Billing and Payment terms and conditions. If full payment is not received on a timely basis, then any license preliminarily granted shall be deemed automatically revoked and shall be void as if never granted. Further, in the event that you breach any of these terms and conditions or any of CCC's Billing and Payment terms and conditions, the license is automatically revoked and shall be void as if never granted. Use of materials as described in a revoked license, as well as any use of the materials beyond the scope of an unrevoked license, may constitute copyright infringement and publisher reserves the right to take any and all action to protect its copyright in the materials.

9. Warranties: Publisher makes no representations or warranties with respect to the licensed material.

10. Indemnity: You hereby indemnify and agree to hold harmless publisher and CCC, and their respective officers, directors, employees and agents, from and against any and all claims arising out of your use of the licensed material other than as specifically authorized pursuant to this license.

11. No Transfer of License: This license is personal to you and may not be sublicensed, assigned, or transferred by you to any other person without publisher's written permission.

12. No Amendment Except in Writing: This license may not be amended except in a writing signed by both parties (or, in the case of publisher, by CCC on publisher's behalf).

13. Objection to Contrary Terms: Publisher hereby objects to any terms contained in any purchase order, acknowledgment, check endorsement or other writing prepared by you, which terms are inconsistent with these terms and conditions or CCC's Billing and Payment terms and conditions. These terms and conditions, together with CCC's Billing and Payment terms and conditions (which are incorporated herein), comprise the entire agreement between you and publisher (and CCC) concerning this licensing transaction. In the event of any conflict between your obligations established by these terms and conditions and those established by CCC's Billing and Payment terms and conditions, these terms and conditions shall control.

14. Revocation: Elsevier or Copyright Clearance Center may deny the permissions described in this License at their sole discretion, for any reason or no reason, with a full refund payable to you. Notice of such denial will be made using the contact information provided by you. Failure to receive such notice will not alter or invalidate the denial. In no event will Elsevier or Copyright Clearance Center be responsible or liable for any costs, expenses or damage incurred by you as a result of a denial of your permission request, other than a refund of the amount(s) paid by you to Elsevier and/or Copyright Clearance Center for denied permissions.

\section{LIMITED LICENSE}

The following terms and conditions apply to specific license types:

15. Translation: This permission is granted for non-exclusive world English rights only unless your license was granted for translation rights. If you licensed translation rights you 
may only translate this content into the languages you requested. A professional translator must perform all translations and reproduce the content word for word preserving the integrity of the article. If this license is to re-use 1 or 2 figures then permission is granted for non-exclusive world rights in all languages.

16. Website: The following terms and conditions apply to electronic reserve and author websites:

Electronic reserve: If licensed material is to be posted to website, the web site is to be password-protected and made available only to bona fide students registered on a relevant course if:

This license was made in connection with a course,

This permission is granted for 1 year only. You may obtain a license for future website posting,

All content posted to the web site must maintain the copyright information line on the bottom of each image,

A hyper-text must be included to the Homepage of the journal from which you are licensing at http://www.sciencedirect.com/science/journal/xxxxx or the Elsevier homepage for books at http://www.elsevier.com, and

Central Storage: This license does not include permission for a scanned version of the material to be stored in a central repository such as that provided by Heron/XanEdu.

17. Author website for journals with the following additional clauses:

This permission is granted for 1 year only. You may obtain a license for future website posting,

All content posted to the web site must maintain the copyright information line on the bottom of each image, and

The permission granted is limited to the personal version of your paper. You are not allowed to download and post the published electronic version of your article (whether PDF or HTML, proof or final version), nor may you scan the printed edition to create an electronic version,

A hyper-text must be included to the Homepage of the journal from which you are licensing at http://www.sciencedirect.com/science/journal/xxxxx , or the Elsevier homepage for books at http://www.elsevier.com and

Central Storage: This license does not include permission for a scanned version of the material to be stored in a central repository such as that provided by Heron/XanEdu.

18. Author website for books with the following additional clauses:

Authors are permitted to place a brief summary of their work online only.

A hyper-text must be included to the Elsevier homepage at http://www.elsevier.com This permission is granted for 1 year only. You may obtain a license for future website posting,

All content posted to the web site must maintain the copyright information line on the bottom of each image, and

The permission granted is limited to the personal version of your paper. You are not allowed to download and post the published electronic version of your article (whether PDF or HTML, proof or final version), nor may you scan the printed edition to create an electronic version,

A hyper-text must be included to the Homepage of the journal from which you are licensing at http://www.sciencedirect.com/science/journal/xxxxx, or the Elsevier homepage for books at http://www.elsevier.com and 
Central Storage: This license does not include permission for a scanned version of the material to be stored in a central repository such as that provided by Heron/XanEdu.

19. Website (regular and for author): "A hyper-text must be included to the Homepage of the journal from which you are licensing at

http://www.sciencedirect.com/science/journal/xxxxx."

20. Thesis/Dissertation: If your license is for use in a thesis/dissertation your thesis may be submitted to your institution in either print or electronic form. Should your thesis be published commercially, please reapply for permission. These requirements include permission for the Library and Archives of Canada to supply single copies, on demand, of the complete thesis and include permission for UMI to supply single copies, on demand, of the complete thesis. Should your thesis be published commercially, please reapply for permission.

v1.2

\section{Other conditions:}

None

https://s100.copyright.com/App/PrintableLicenseFrame.jsp?publisherID=70\&licenseID=2 $\ldots \quad$ 7/19/2008 
ELSEVIER LICENSE TERMS AND CONDITIONS

Aug 30, 2008

This is a License Agreement between Sara M Leifer ("You") and Elsevier ("Elsevier"). The license consists of your order details, the terms and conditions provided by Elsevier, and the payment terms and conditions.

\begin{tabular}{|c|c|}
\hline Supplier & $\begin{array}{l}\text { Elsevier Limited } \\
\text { The Boulevard,Langford Lane } \\
\text { Kidlington,Oxford,OX5 1GB,UK }\end{array}$ \\
\hline Registered Company Number & 1982084 \\
\hline Customer name & Sara M Leifer \\
\hline \multirow[t]{2}{*}{ Customer address } & 141 Valley View Terrace \\
\hline & Mission Viejo, CA 92692 \\
\hline License Number & 2019051025246 \\
\hline License date & Aug 30, 2008 \\
\hline Licensed content publisher & Elsevier \\
\hline Licensed content publication & $\begin{array}{l}\text { Journal of the American College of } \\
\text { Cardiology }\end{array}$ \\
\hline Licensed content title & Selection of coronary stents \\
\hline Licensed content author & $\begin{array}{l}\text { Antonio Colombo, Goran } \\
\text { Stankovic and Jeffrey W. Moses }\end{array}$ \\
\hline Licensed content date & 18 September 2002 \\
\hline \multicolumn{2}{|l|}{ Volume number } \\
\hline \multicolumn{2}{|l|}{ Issue number } \\
\hline Pages & 0 \\
\hline Type of Use & Thesis / Dissertation \\
\hline Portion & Figures/table/illustration/abstracts \\
\hline Portion Quantity & 1 \\
\hline Format & Both print and electronic \\
\hline You are an author of the Elsevier article & No \\
\hline Are you translating? & No \\
\hline \multicolumn{2}{|l|}{ Purchase order number } \\
\hline Expected publication date & Sep 2008 \\
\hline Elsevier VAT number & GB 494627212 \\
\hline Permissions price & 0.00 USD \\
\hline Value added tax $0.0 \%$ & 0.00 USD \\
\hline Total & 0.00 USD \\
\hline
\end{tabular}

https://s100.copyright.com/CustomerAdmin/PLF.jsp?IID=2008081_1220142185246 
Terms and Conditions

\section{INTRODUCTION}

1. The publisher for this copyrighted material is Elsevier. By clicking "accept" in connection with completing this licensing transaction, you agree that the following terms and conditions apply to this transaction (along with the Billing and Payment terms and conditions established by Copyright Clearance Center, Inc. ("CCC"), at the time that you opened your Rightslink account and that are available at any time at $<\mathrm{http}: / /$ myaccount.copyright.com>).

\section{GENERAL TERMS}

2. Elsevier hereby grants you permission to reproduce the aforementioned material subject to the terms and conditions indicated.

3. Acknowledgement: If any part of the material to be used (for example, figures) has appeared in our publication with credit or acknowledgement to another source, permission must also be sought from that source. If such permission is not obtained then that material may not be included in your publication/copies. Suitable acknowledgement to the source must be made, either as a footnote or in a reference list at the end of your publication, as follows:

"Reprinted from Publication title, Vol /edition number, Author(s), Title of article / title of chapter, Pages No., Copyright (Year), with permission from Elsevier [OR APPLICABLE SOCIETY COPYRIGHT OWNER]." Also Lancet special credit - "Reprinted from The Lancet, Vol. number, Author(s), Title of article, Pages No., Copyright (Year), with permission from Elsevier."

4. Reproduction of this material is confined to the purpose and/or media for which permission is hereby given.

5. Altering/Modifying Material: Not Permitted. However figures and illustrations may be altered/adapted minimally to serve your work. Any other abbreviations, additions, deletions and/or any other alterations shall be made only with prior written authorization of Elsevier Ltd. (Please contact Elsevier at permissions@elsevier.com)

6. If the permission fee for the requested use of our material is waived in this instance, please be advised that your future requests for Elsevier materials may attract a fee.

7. Reservation of Rights: Publisher reserves all rights not specifically granted in the combination of (i) the license details provided by you and accepted in the course of this licensing transaction, (ii) these terms and conditions and (iii) CCC's Billing and Payment terms and conditions.

8. License Contingent Upon Payment: While you may exercise the rights licensed immediately upon issuance of the license at the end of the licensing process for the transaction, provided that you have disclosed complete and accurate details of your proposed use, no license is finally effective unless and until full payment is received from you (either by publisher or by CCC) as provided in CCC's Billing and Payment terms and conditions. If 
full payment is not received on a timely basis, then any license preliminarily granted shall be deemed automatically revoked and shall be void as if never granted. Further, in the event that you breach any of these terms and conditions or any of CCC's Billing and Payment terms and conditions, the license is automatically revoked and shall be void as if never granted. Use of materials as described in a revoked license, as well as any use of the materials beyond the scope of an unrevoked license, may constitute copyright infringement and publisher reserves the right to take any and all action to protect its copyright in the materials.

9. Warranties: Publisher makes no representations or warranties with respect to the licensed material.

10. Indemnity: You hereby indemnify and agree to hold harmless publisher and CCC, and their respective officers, directors, employees and agents, from and against any and all claims arising out of your use of the licensed material other than as specifically authorized pursuant to this license.

11. No Transfer of License: This license is personal to you and may not be sublicensed, assigned, or transferred by you to any other person without publisher's written permission.

12. No Amendment Except in Writing: This license may not be amended except in a writing signed by both parties (or, in the case of publisher, by CCC on publisher's behalf).

13. Objection to Contrary Terms: Publisher hereby objects to any terms contained in any purchase order, acknowledgment, check endorsement or other writing prepared by you, which terms are inconsistent with these terms and conditions or CCC's Billing and Payment terms and conditions. These terms and conditions, together with CCC's Billing and Payment terms and conditions (which are incorporated herein), comprise the entire agreement between you and publisher (and CCC) concerning this licensing transaction. In the event of any conflict between your obligations established by these terms and conditions and those established by CCC's Billing and Payment terms and conditions, these terms and conditions shall control.

14. Revocation: Elsevier or Copyright Clearance Center may deny the permissions described in this License at their sole discretion, for any reason or no reason, with a full refund payable to you. Notice of such denial will be made using the contact information provided by you. Failure to receive such notice will not alter or invalidate the denial. In no event will Elsevier or Copyright Clearance Center be responsible or liable for any costs, expenses or damage incurred by you as a result of a denial of your permission request, other than a refund of the amount(s) paid by you to Elsevier and/or Copyright Clearance Center for denied permissions.

\section{LIMITED LICENSE}

The following terms and conditions apply to specific license types:

15. Translation: This permission is granted for non-exclusive world English rights only unless your license was granted for translation rights. If you licensed translation rights you may only translate this content into the languages you requested. A professional translator must perform all translations and reproduce the content word for word preserving the 
integrity of the article. If this license is to re-use 1 or 2 figures then permission is granted for non-exclusive world rights in all languages.

16. Website: The following terms and conditions apply to electronic reserve and author websites:

Electronic reserve: If licensed material is to be posted to website, the web site is to be password-protected and made available only to bona fide students registered on a relevant course if:

This license was made in connection with a course,

This permission is granted for 1 year only. You may obtain a license for future website posting,

All content posted to the web site must maintain the copyright information line on the bottom of each image,

A hyper-text must be included to the Homepage of the journal from which you are licensing at http://www.sciencedirect.com/science/journal/xxxxx or the Elsevier homepage for books at http://www.elsevier.com , and

Central Storage: This license does not include permission for a scanned version of the material to be stored in a central repository such as that provided by Heron/XanEdu.

17. Author website for journals with the following additional clauses:

This permission is granted for 1 year only. You may obtain a license for future website posting,

All content posted to the web site must maintain the copyright information line on the bottom of each image, and

The permission granted is limited to the personal version of your paper. You are not allowed to download and post the published electronic version of your article (whether PDF or HTML, proof or final version), nor may you scan the printed edition to create an electronic version,

A hyper-text must be included to the Homepage of the journal from which you are licensing at http://www.sciencedirect.com/science/journal/xxxxx, or the Elsevier homepage for books at http://www.elsevier.com and

Central Storage: This license does not include permission for a scanned version of the material to be stored in a central repository such as that provided by Heron/XanEdu.

18. Author website for books with the following additional clauses:

Authors are permitted to place a brief summary of their work online only.

A hyper-text must be included to the Elsevier homepage at http://www.elsevier.com This permission is granted for 1 year only. You may obtain a license for future website posting,

All content posted to the web site must maintain the copyright information line on the bottom of each image, and

The permission granted is limited to the personal version of your paper. You are not allowed to download and post the published electronic version of your article (whether PDF or HTML, proof or final version), nor may you scan the printed edition to create an electronic version,

A hyper-text must be included to the Homepage of the journal from which you are licensing at http://www.sciencedirect.com/science/journal/xxxxx, or the Elsevier homepage for books at http://www.elsevier.com and

Central Storage: This license does not include permission for a scanned version of the material to be stored in a central repository such as that provided by Heron/XanEdu. 
19. Website (regular and for author): "A hyper-text must be included to the Homepage of the journal from which you are licensing at

http://www.sciencedirect.com/science/journal/xxxxx."

20. Thesis/Dissertation: If your license is for use in a thesis/dissertation your thesis may be submitted to your institution in either print or electronic form. Should your thesis be published commercially, please reapply for permission. These requirements include permission for the Library and Archives of Canada to supply single copies, on demand, of the complete thesis and include permission for UMI to supply single copies, on demand, of the complete thesis. Should your thesis be published commercially, please reapply for permission.

v1.2

21. Other conditions:

None 
ELSEVIER LICENSE TERMS AND CONDITIONS

Aug 31, 2008

This is a License Agreement between Sara M Leifer ("You") and Elsevier ("Elsevier"). The license consists of your order details, the terms and conditions provided by Elsevier, and the payment terms and conditions.

\begin{tabular}{|c|c|}
\hline Supplier & $\begin{array}{l}\text { Elsevier Limited } \\
\text { The Boulevard,Langford Lane } \\
\text { Kidlington,Oxford,OX5 1GB,UK }\end{array}$ \\
\hline Registered Company Number & 1982084 \\
\hline Customer name & Sara M Leifer \\
\hline \multirow[t]{2}{*}{ Customer address } & 141 Valley View Terrace \\
\hline & Mission Viejo, CA 92692 \\
\hline License Number & 2019571043165 \\
\hline License date & Aug 31,2008 \\
\hline Licensed content publisher & Elsevier \\
\hline Licensed content publication & The Asia Pacific Heart Journal \\
\hline Licensed content title & $\begin{array}{l}\text { Intracoronary stenting: From } \\
\text { concept to custom }\end{array}$ \\
\hline Licensed content author & Peter Ruygrok \\
\hline Licensed content date & May 1997 \\
\hline \multicolumn{2}{|l|}{ Volume number } \\
\hline \multicolumn{2}{|l|}{ Issue number } \\
\hline Pages & 0 \\
\hline Type of Use & Thesis / Dissertation \\
\hline Portion & Figures/table/illustration/abstracts \\
\hline Portion Quantity & 2 \\
\hline Format & Both print and electronic \\
\hline You are an author of the Elsevier article & No \\
\hline Are you translating? & No \\
\hline \multicolumn{2}{|l|}{ Purchase order number } \\
\hline Expected publication date & Oct 2008 \\
\hline Elsevier VAT number & GB 494627212 \\
\hline Permissions price & 0.00 USD \\
\hline Value added tax $0.0 \%$ & 0.00 USD \\
\hline Total & 0.00 USD \\
\hline
\end{tabular}

https://s100.copyright.com/App/PrintableLicenseFrame.jsp?publisherID=70\&licenseID=2 $\ldots \quad 8 / 31 / 2008$ 


\section{INTRODUCTION}

1. The publisher for this copyrighted material is Elsevier. By clicking "accept" in connection with completing this licensing transaction, you agree that the following terms and conditions apply to this transaction (along with the Billing and Payment terms and conditions established by Copyright Clearance Center, Inc. ("CCC"), at the time that you opened your Rightslink account and that are available at any time at $<\mathrm{http}: / /$ myaccount.copyright.com $>$ ).

\section{GENERAL TERMS}

2. Elsevier hereby grants you permission to reproduce the aforementioned material subject to the terms and conditions indicated.

3. Acknowledgement: If any part of the material to be used (for example, figures) has appeared in our publication with credit or acknowledgement to another source, permission must also be sought from that source. If such permission is not obtained then that material may not be included in your publication/copies. Suitable acknowledgement to the source must be made, either as a footnote or in a reference list at the end of your publication, as follows:

"Reprinted from Publication title, Vol /edition number, Author(s), Title of article / title of chapter, Pages No., Copyright (Year), with permission from Elsevier [OR APPLICABLE SOCIETY COPYRIGHT OWNER]." Also Lancet special credit - "Reprinted from The Lancet, Vol. number, Author(s), Title of article, Pages No., Copyright (Year), with permission from Elsevier."

4. Reproduction of this material is confined to the purpose and/or media for which permission is hereby given.

5. Altering/Modifying Material: Not Permitted. However figures and illustrations may be altered/adapted minimally to serve your work. Any other abbreviations, additions, deletions and/or any other alterations shall be made only with prior written authorization of Elsevier Ltd. (Please contact Elsevier at permissions@elsevier.com)

6. If the permission fee for the requested use of our material is waived in this instance, please be advised that your future requests for Elsevier materials may attract a fee.

7. Reservation of Rights: Publisher reserves all rights not specifically granted in the combination of (i) the license details provided by you and accepted in the course of this licensing transaction, (ii) these terms and conditions and (iii) CCC's Billing and Payment terms and conditions.

8. License Contingent Upon Payment: While you may exercise the rights licensed immediately upon issuance of the license at the end of the licensing process for the transaction, provided that you have disclosed complete and accurate details of your proposed use, no license is finally effective unless and until full payment is received from you (either by publisher or by CCC) as provided in CCC's Billing and Payment terms and conditions. If full payment is not received on a timely basis, then any license preliminarily granted shall be deemed automatically revoked and shall be void as if never granted. Further, in the event 
that you breach any of these terms and conditions or any of CCC's Billing and Payment terms and conditions, the license is automatically revoked and shall be void as if never granted. Use of materials as described in a revoked license, as well as any use of the materials beyond the scope of an unrevoked license, may constitute copyright infringement and publisher reserves the right to take any and all action to protect its copyright in the materials.

9. Warranties: Publisher makes no representations or warranties with respect to the licensed material.

10. Indemnity: You hereby indemnify and agree to hold harmless publisher and CCC, and their respective officers, directors, employees and agents, from and against any and all claims arising out of your use of the licensed material other than as specifically authorized pursuant to this license.

11. No Transfer of License: This license is personal to you and may not be sublicensed, assigned, or transferred by you to any other person without publisher's written permission.

12. No Amendment Except in Writing: This license may not be amended except in a writing signed by both parties (or, in the case of publisher, by CCC on publisher's behalf).

13. Objection to Contrary Terms: Publisher hereby objects to any terms contained in any purchase order, acknowledgment, check endorsement or other writing prepared by you, which terms are inconsistent with these terms and conditions or CCC's Billing and Payment terms and conditions. These terms and conditions, together with CCC's Billing and Payment terms and conditions (which are incorporated herein), comprise the entire agreement between you and publisher (and CCC) concerning this licensing transaction. In the event of any conflict between your obligations established by these terms and conditions and those established by CCC's Billing and Payment terms and conditions, these terms and conditions shall control.

14. Revocation: Elsevier or Copyright Clearance Center may deny the permissions described in this License at their sole discretion, for any reason or no reason, with a full refund payable to you. Notice of such denial will be made using the contact information provided by you. Failure to receive such notice will not alter or invalidate the denial. In no event will Elsevier or Copyright Clearance Center be responsible or liable for any costs, expenses or damage incurred by you as a result of a denial of your permission request, other than a refund of the amount(s) paid by you to Elsevier and/or Copyright Clearance Center for denied permissions.

\section{LIMITED LICENSE}

The following terms and conditions apply to specific license types:

15. Translation: This permission is granted for non-exclusive world English rights only unless your license was granted for translation rights. If you licensed translation rights you may only translate this content into the languages you requested. A professional translator must perform all translations and reproduce the content word for word preserving the integrity of the article. If this license is to re-use 1 or 2 figures then permission is granted for non-exclusive world rights in all languages. 
16. Website: The following terms and conditions apply to electronic reserve and author websites:

Electronic reserve: If licensed material is to be posted to website, the web site is to be password-protected and made available only to bona fide students registered on a relevant course if:

This license was made in connection with a course,

This permission is granted for 1 year only. You may obtain a license for future website posting,

All content posted to the web site must maintain the copyright information line on the bottom of each image,

A hyper-text must be included to the Homepage of the journal from which you are licensing at http://www.sciencedirect.com/science/journal/xxxxx or the Elsevier homepage for books at http://www.elsevier.com , and

Central Storage: This license does not include permission for a scanned version of the material to be stored in a central repository such as that provided by Heron/XanEdu.

17. Author website for journals with the following additional clauses:

This permission is granted for 1 year only. You may obtain a license for future website posting,

All content posted to the web site must maintain the copyright information line on the bottom of each image, and

The permission granted is limited to the personal version of your paper. You are not allowed to download and post the published electronic version of your article (whether PDF or HTML, proof or final version), nor may you scan the printed edition to create an electronic version,

A hyper-text must be included to the Homepage of the journal from which you are licensing at http://www.sciencedirect.com/science/journal/xxxxx, or the Elsevier homepage for books at http://www.elsevier.com and

Central Storage: This license does not include permission for a scanned version of the material to be stored in a central repository such as that provided by Heron/XanEdu.

18. Author website for books with the following additional clauses:

Authors are permitted to place a brief summary of their work online only. A hyper-text must be included to the Elsevier homepage at http://www.elsevier.com This permission is granted for 1 year only. You may obtain a license for future website posting,

All content posted to the web site must maintain the copyright information line on the bottom of each image, and

The permission granted is limited to the personal version of your paper. You are not allowed to download and post the published electronic version of your article (whether PDF or HTML, proof or final version), nor may you scan the printed edition to create an electronic version,

A hyper-text must be included to the Homepage of the journal from which you are licensing at http://www.sciencedirect.com/science/journal/xxxxx , or the Elsevier homepage for books at http://www.elsevier.com and

Central Storage: This license does not include permission for a scanned version of the material to be stored in a central repository such as that provided by Heron/XanEdu. 
19. Website (regular and for author): "A hyper-text must be included to the Homepage of the journal from which you are licensing at

http://www.sciencedirect.com/science/journal/xxxxx."

20. Thesis/Dissertation: If your license is for use in a thesis/dissertation your thesis may be submitted to your institution in either print or electronic form. Should your thesis be published commercially, please reapply for permission. These requirements include permission for the Library and Archives of Canada to supply single copies, on demand, of the complete thesis and include permission for UMI to supply single copies, on demand, of the complete thesis. Should your thesis be published commercially, please reapply for permission.

v1.2

21. Other conditions:

None

https://s100.copyright.com/App/PrintableLicenseFrame.jsp?publisherID=70\&licenseID $=2 \ldots \quad 8 / 31 / 2008$ 
This is a License Agreement between Sara M Leifer ("You") and Elsevier ("Elsevier"). The license consists of your order details, the terms and conditions provided by Elsevier, and the payment terms and conditions.

Supplier

Registered Company Number

Customer name

Customer address

License Number

License date

Licensed content publisher

Licensed content publication

Licensed content title

Licensed content author

Licensed content date

Volume number

Issue number

Pages

Type of Use

Portion

Portion Quantity

Format

You are an author of the Elsevier article

Are you translating?

Purchase order number

Expected publication date

Elsevier VAT number

Permissions price

Value added tax $0.0 \%$
Elsevier Limited

The Boulevard, Langford Lane

Kidlington,Oxford,OX5 1GB,UK

1982084

Sara M Leifer

141 Valley View Terrace

Mission Viejo, CA 92692

2023470921043

Sep 07, 2008

Elsevier

Journal of the American College of Cardiology

Contemporary Stent Treatment of Coronary Bifurcations

Ioannis Iakovou, Lei $\mathrm{Ge}$ and Antonio Colombo

18 October 2005

Thesis / Dissertation

Figures/table/illustration/abstracts

Both print and electronic

No

No

Oct 2008

GB 494627212

0.00 USD

0.00 USD

https://s100.copyright.com/App/PrintableLicenseFrame.jsp?publisherID=70\&licenseID=20 .. 9/6/2008 
Terms and Conditions

\section{INTRODUCTION}

1. The publisher for this copyrighted material is Elsevier. By clicking "accept" in connection with completing this licensing transaction, you agree that the following terms and conditions apply to this transaction (along with the Billing and Payment terms and conditions established by Copyright Clearance Center, Inc. ("CCC"), at the time that you opened your Rightslink account and that are available at any time at $<$ http://myaccount.copyright.com $>$ ).

\section{GENERAL TERMS}

2. Elsevier hereby grants you permission to reproduce the aforementioned material subject to the terms and conditions indicated.

3. Acknowledgement: If any part of the material to be used (for example, figures) has appeared in our publication with credit or acknowledgement to another source, permission must also be sought from that source. If such permission is not obtained then that material may not be included in your publication/copies. Suitable acknowledgement to the source must be made, either as a footnote or in a reference list at the end of your publication, as follows:

"Reprinted from Publication title, Vol /edition number, Author(s), Title of article / title of chapter, Pages No., Copyright (Year), with permission from Elsevier [OR APPLICABLE SOCIETY COPYRIGHT OWNER]." Also Lancet special credit - "Reprinted from The Lancet, Vol. number, Author(s), Title of article, Pages No., Copyright (Year), with permission from Elsevier."

4. Reproduction of this material is confined to the purpose and/or media for which permission is hereby given.

5. Altering/Modifying Material: Not Permitted. However figures and illustrations may be altered/adapted minimally to serve your work. Any other abbreviations, additions, deletions and/or any other alterations shall be made only with prior written authorization of Elsevier Ltd. (Please contact Elsevier at permissions@elsevier.com)

6. If the permission fee for the requested use of our material is waived in this instance, please be advised that your future requests for Elsevier materials may attract a fee.

7. Reservation of Rights: Publisher reserves all rights not specifically granted in the combination of (i) the license details provided by you and accepted in the course of this licensing transaction, (ii) these terms and conditions and (iii) CCC's Billing and Payment terms and conditions.

8. License Contingent Upon Payment: While you may exercise the rights licensed immediately upon issuance of the license at the end of the licensing process for the transaction, provided that you have disclosed complete and accurate details of your proposed use, no license is finally effective unless and until full payment is received from you (either

https://s100.copyright.com/App/PrintableLicenseFrame.jsp?publisherID=70\&licenseID=20_. 9/6/2008 
by publisher or by $\mathrm{CCC}$ ) as provided in CCC's Billing and Payment terms and conditions. If full payment is not received on a timely basis, then any license preliminarily granted shall be deemed automatically revoked and shall be void as if never granted. Further, in the event that you breach any of these terms and conditions or any of CCC's Billing and Payment terms and conditions, the license is automatically revoked and shall be void as if never granted. Use of materials as described in a revoked license, as well as any use of the materials beyond the scope of an unrevoked license, may constitute copyright infringement and publisher reserves the right to take any and all action to protect its copyright in the materials.

9. Warranties: Publisher makes no representations or warranties with respect to the licensed material.

10. Indemnity: You hereby indemnify and agree to hold harmless publisher and CCC, and their respective officers, directors, employees and agents, from and against any and all claims arising out of your use of the licensed material other than as specifically authorized pursuant to this license.

11. No Transfer of License: This license is personal to you and may not be sublicensed, assigned, or transferred by you to any other person without publisher's written permission.

12. No Amendment Except in Writing: This license may not be amended except in a writing signed by both parties (or, in the case of publisher, by CCC on publisher's behalf).

13. Objection to Contrary Terms: Publisher hereby objects to any terms contained in any purchase order, acknowledgment, check endorsement or other writing prepared by you, which terms are inconsistent with these terms and conditions or CCC's Billing and Payment terms and conditions. These terms and conditions, together with CCC's Billing and Payment terms and conditions (which are incorporated herein), comprise the entire agreement between you and publisher (and CCC) concerning this licensing transaction. In the event of any conflict between your obligations established by these terms and conditions and those established by CCC's Billing and Payment terms and conditions, these terms and conditions shall control.

14. Revocation: Elsevier or Copyright Clearance Center may deny the permissions described in this License at their sole discretion, for any reason or no reason, with a full refund payable to you. Notice of such denial will be made using the contact information provided by you. Failure to receive such notice will not alter or invalidate the denial. In no event will Elsevier or Copyright Clearance Center be responsible or liable for any costs, expenses or damage incurred by you as a result of a denial of your permission request, other than a refund of the amount(s) paid by you to Elsevier and/or Copyright Clearance Center for denied permissions.

\section{LIMITED LICENSE}

The following terms and conditions apply to specific license types:

15. Translation: This permission is granted for non-exclusive world English rights only unless your license was granted for translation rights. If you licensed translation rights you may only translate this content into the languages you requested. A professional translator

https://s100.copyright.com/App/PrintableLicenseFrame.jsp?publisherID=70\&licenseID=20 .. 9/6/2008 
must perform all translations and reproduce the content word for word preserving the integrity of the article. If this license is to re-use 1 or 2 figures then permission is granted for non-exclusive world rights in all languages.

16. Website: The following terms and conditions apply to electronic reserve and author websites:

Electronic reserve: If licensed material is to be posted to website, the web site is to be password-protected and made available only to bona fide students registered on a relevant course if:

This license was made in connection with a course,

This permission is granted for 1 year only. You may obtain a license for future website posting,

All content posted to the web site must maintain the copyright information line on the bottom of each image,

A hyper-text must be included to the Homepage of the journal from which you are licensing at http://www.sciencedirect.com/science/journal/xxxxx or the Elsevier homepage for books at http://www.elsevier.com , and

Central Storage: This license does not include permission for a scanned version of the material to be stored in a central repository such as that provided by Heron/XanEdu.

17. Author website for journals with the following additional clauses:

This permission is granted for 1 year only. You may obtain a license for future website posting,

All content posted to the web site must maintain the copyright information line on the bottom of each image, and

The permission granted is limited to the personal version of your paper. You are not allowed to download and post the published electronic version of your article (whether PDF or HTML, proof or final version), nor may you scan the printed edition to create an electronic version,

A hyper-text must be included to the Homepage of the journal from which you are licensing at http://www.sciencedirect.com/science/journal/xxxxx, or the Elsevier homepage for books at http://www.elsevier.com and

Central Storage: This license does not include permission for a scanned version of the material to be stored in a central repository such as that provided by Heron/XanEdu.

18. Author website for books with the following additional clauses:

Authors are permitted to place a brief summary of their work online only.

A hyper-text must be included to the Elsevier homepage at http://www.elsevier.com This permission is granted for 1 year only. You may obtain a license for future website posting,

All content posted to the web site must maintain the copyright information line on the bottom of each image, and

The permission granted is limited to the personal version of your paper. You are not allowed to download and post the published electronic version of your article (whether PDF or HTML, proof or final version), nor may you scan the printed edition to create an electronic version,

A hyper-text must be included to the Homepage of the journal from which you are licensing at http://www.sciencedirect.com/science/journal/xxxxx , or the Elsevier homepage for books at http://www.elsevier.com and

Central Storage: This license does not include permission for a scanned version of the 
material to be stored in a central repository such as that provided by Heron/XanEdu.

19. Website (regular and for author): "A hyper-text must be included to the Homepage of the journal from which you are licensing at

http://www.sciencedirect.com/science/journal/xxxxx."

20. Thesis/Dissertation: If your license is for use in a thesis/dissertation your thesis may be submitted to your institution in either print or electronic form. Should your thesis be published commercially, please reapply for permission. These requirements include permission for the Library and Archives of Canada to supply single copies, on demand, of the complete thesis and include permission for UMI to supply single copies, on demand, of the complete thesis. Should your thesis be published commercially, please reapply for permission.

$\mathrm{v} 1.2$

21. Other conditions:

None

https://s100.copyright.com/App/PrintableLicenseFrame.jsp?publisherID=70\&licenseID=20_. 9/6/2008 
ELSEVIER LICENSE TERMS AND CONDITIONS

Sep 13, 2008

This is a License Agreement between Sara M Leifer ("You") and Elsevier ("Elsevier"). The license consists of your order details, the terms and conditions provided by Elsevier, and the payment terms and conditions.

\begin{tabular}{|c|c|}
\hline Supplier & $\begin{array}{l}\text { Elsevier Limited } \\
\text { The Boulevard,Langford Lane } \\
\text { Kidlington,Oxford,OX5 1GB,UK }\end{array}$ \\
\hline Registered Company Number & 1982084 \\
\hline Customer name & Sara M Leifer \\
\hline \multirow[t]{2}{*}{ Customer address } & 141 Valley View Terrace \\
\hline & Mission Viejo, CA 92692 \\
\hline License Number & 2027330951654 \\
\hline License date & Sep 13, 2008 \\
\hline Licensed content publisher & Elsevier \\
\hline Licensed content publication & Advanced Drug Delivery Reviews \\
\hline Licensed content title & $\begin{array}{l}\text { Role of stent design and coatings } \\
\text { on restenosis and thrombosis }\end{array}$ \\
\hline Licensed content author & $\begin{array}{l}\text { Hidehiko Hara, Masato Nakamura, } \\
\text { Julio C. Palmaz and Robert S. } \\
\text { Schwartz }\end{array}$ \\
\hline Licensed content date & 3 June 2006 \\
\hline Volume number & 58 \\
\hline Issue number & 3 \\
\hline Pages & 10 \\
\hline Type of Use & Thesis / Dissertation \\
\hline Portion & Figures/table/illustration/abstracts \\
\hline Portion Quantity & 1 \\
\hline Format & Both print and electronic \\
\hline You are an author of the Elsevier article & No \\
\hline Are you translating? & No \\
\hline \multicolumn{2}{|l|}{ Purchase order number } \\
\hline Expected publication date & Oct 2008 \\
\hline Elsevier VAT number & GB 494627212 \\
\hline Permissions price & 0.00 USD \\
\hline Value added tax $0.0 \%$ & 0.00 USD \\
\hline
\end{tabular}

https://s100.copyright.com/App/PrintableLicenseFrame.jsp?publisherID=70\&licenseID=2 $\ldots \quad$ 9/13/2008 
Terms and Conditions

\section{INTRODUCTION}

1. The publisher for this copyrighted material is Elsevier. By clicking "accept" in connection with completing this licensing transaction, you agree that the following terms and conditions apply to this transaction (along with the Billing and Payment terms and conditions established by Copyright Clearance Center, Inc. ("CCC"), at the time that you opened your Rightslink account and that are available at any time at $<\mathrm{http}: / /$ myaccount.copyright.com>).

\section{GENERAL TERMS}

2. Elsevier hereby grants you permission to reproduce the aforementioned material subject to the terms and conditions indicated.

3. Acknowledgement: If any part of the material to be used (for example, figures) has appeared in our publication with credit or acknowledgement to another source, permission must also be sought from that source. If such permission is not obtained then that material may not be included in your publication/copies. Suitable acknowledgement to the source must be made, either as a footnote or in a reference list at the end of your publication, as follows:

"Reprinted from Publication title, Vol /edition number, Author(s), Title of article / title of chapter, Pages No., Copyright (Year), with permission from Elsevier [OR APPLICABLE SOCIETY COPYRIGHT OWNER]." Also Lancet special credit - "Reprinted from The Lancet, Vol. number, Author(s), Title of article, Pages No., Copyright (Year), with permission from Elsevier."

4. Reproduction of this material is confined to the purpose and/or media for which permission is hereby given.

5. Altering/Modifying Material: Not Permitted. However figures and illustrations may be altered/adapted minimally to serve your work. Any other abbreviations, additions, deletions and/or any other alterations shall be made only with prior written authorization of Elsevier Ltd. (Please contact Elsevier at permissions@elsevier.com)

6. If the permission fee for the requested use of our material is waived in this instance, please be advised that your future requests for Elsevier materials may attract a fee.

7. Reservation of Rights: Publisher reserves all rights not specifically granted in the combination of (i) the license details provided by you and accepted in the course of this licensing transaction, (ii) these terms and conditions and (iii) CCC's Billing and Payment terms and conditions.

8. License Contingent Upon Payment: While you may exercise the rights licensed immediately upon issuance of the license at the end of the licensing process for the transaction, provided that you have disclosed complete and accurate details of your proposed use, no license is finally effective unless and until full payment is received from you (either 
by publisher or by $\mathrm{CCC}$ ) as provided in CCC's Billing and Payment terms and conditions. If full payment is not received on a timely basis, then any license preliminarily granted shall be deemed automatically revoked and shall be void as if never granted. Further, in the event that you breach any of these terms and conditions or any of CCC's Billing and Payment terms and conditions, the license is automatically revoked and shall be void as if never granted. Use of materials as described in a revoked license, as well as any use of the materials beyond the scope of an unrevoked license, may constitute copyright infringement and publisher reserves the right to take any and all action to protect its copyright in the materials.

9. Warranties: Publisher makes no representations or warranties with respect to the licensed material.

10. Indemnity: You hereby indemnify and agree to hold harmless publisher and CCC, and their respective officers, directors, employees and agents, from and against any and all claims arising out of your use of the licensed material other than as specifically authorized pursuant to this license.

11. No Transfer of License: This license is personal to you and may not be sublicensed, assigned, or transferred by you to any other person without publisher's written permission.

12. No Amendment Except in Writing: This license may not be amended except in a writing signed by both parties (or, in the case of publisher, by CCC on publisher's behalf).

13. Objection to Contrary Terms: Publisher hereby objects to any terms contained in any purchase order, acknowledgment, check endorsement or other writing prepared by you, which terms are inconsistent with these terms and conditions or CCC's Billing and Payment terms and conditions. These terms and conditions, together with CCC's Billing and Payment terms and conditions (which are incorporated herein), comprise the entire agreement between you and publisher (and CCC) concerning this licensing transaction. In the event of any conflict between your obligations established by these terms and conditions and those established by CCC's Billing and Payment terms and conditions, these terms and conditions shall control.

14. Revocation: Elsevier or Copyright Clearance Center may deny the permissions described in this License at their sole discretion, for any reason or no reason, with a full refund payable to you. Notice of such denial will be made using the contact information provided by you. Failure to receive such notice will not alter or invalidate the denial. In no event will Elsevier or Copyright Clearance Center be responsible or liable for any costs, expenses or damage incurred by you as a result of a denial of your permission request, other than a refund of the amount(s) paid by you to Elsevier and/or Copyright Clearance Center for denied permissions.

\section{LIMITED LICENSE}

The following terms and conditions apply to specific license types:

15. Translation: This permission is granted for non-exclusive world English rights only unless your license was granted for translation rights. If you licensed translation rights you may only translate this content into the languages you requested. A professional translator 
must perform all translations and reproduce the content word for word preserving the integrity of the article. If this license is to re-use 1 or 2 figures then permission is granted for non-exclusive world rights in all languages.

16. Website: The following terms and conditions apply to electronic reserve and author websites:

Electronic reserve: If licensed material is to be posted to website, the web site is to be password-protected and made available only to bona fide students registered on a relevant course if:

This license was made in connection with a course,

This permission is granted for 1 year only. You may obtain a license for future website posting,

All content posted to the web site must maintain the copyright information line on the bottom of each image,

A hyper-text must be included to the Homepage of the journal from which you are licensing at http://www.sciencedirect.com/science/journal/xxxxx or the Elsevier homepage for books at http://www.elsevier.com , and

Central Storage: This license does not include permission for a scanned version of the material to be stored in a central repository such as that provided by Heron/XanEdu.

17. Author website for journals with the following additional clauses:

This permission is granted for 1 year only. You may obtain a license for future website posting,

All content posted to the web site must maintain the copyright information line on the bottom of each image, and

The permission granted is limited to the personal version of your paper. You are not allowed to download and post the published electronic version of your article (whether PDF or HTML, proof or final version), nor may you scan the printed edition to create an electronic version,

A hyper-text must be included to the Homepage of the journal from which you are licensing at http://www.sciencedirect.com/science/journal/xxxxx, or the Elsevier homepage for books at http://www.elsevier.com and

Central Storage: This license does not include permission for a scanned version of the material to be stored in a central repository such as that provided by Heron/XanEdu.

18. Author website for books with the following additional clauses:

Authors are permitted to place a brief summary of their work online only.

A hyper-text must be included to the Elsevier homepage at http://www.elsevier.com This permission is granted for 1 year only. You may obtain a license for future website posting,

All content posted to the web site must maintain the copyright information line on the bottom of each image, and

The permission granted is limited to the personal version of your paper. You are not allowed to download and post the published electronic version of your article (whether PDF or HTML, proof or final version), nor may you scan the printed edition to create an electronic version,

A hyper-text must be included to the Homepage of the journal from which you are licensing at http://www.sciencedirect.com/science/journal/xxxxx , or the Elsevier homepage for books at http://www.elsevier.com and

Central Storage: This license does not include permission for a scanned version of the 
material to be stored in a central repository such as that provided by Heron/XanEdu.

19. Website (regular and for author): "A hyper-text must be included to the Homepage of the journal from which you are licensing at

http://www.sciencedirect.com/science/journal/xxxxx."

20. Thesis/Dissertation: If your license is for use in a thesis/dissertation your thesis may be submitted to your institution in either print or electronic form. Should your thesis be published commercially, please reapply for permission. These requirements include permission for the Library and Archives of Canada to supply single copies, on demand, of the complete thesis and include permission for UMI to supply single copies, on demand, of the complete thesis. Should your thesis be published commercially, please reapply for permission.

$\mathrm{v} 1.2$

21. Other conditions:

None

https://s100.copyright.com/App/PrintableLicenseFrame.jsp?publisherID=70\&licenseID=2 $\ldots \quad$ 9/13/2008 
Chabman of the Romet

Gavy $L$ Elis

Prosident

Deniel W. danes, MD, FAHA

Chairman-Elest

David A Jessertand

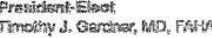

Unmedials Past

mamediake Fast Presidsmi

Reymenat I, Gabons, MD, FALA

Sepretary-Thossurer

Dhane W. Lothentod, CPA

Directors

Danna K. Annat, FhD, FaHA

Shortes Clarabses, WS

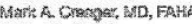

Strewn A. Dennis

Prey Duress

Datrma A. Gaitselse

Thos cornez, Pho

Mark B MoPlstlan, MD, Ph

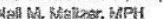

Jucith F, Osson, Es?

Fijph L Saves, MBD, FAHA

Dowien, Spina

Samuet H. Tumer S.

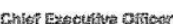

M. Cass Wheoler

Chisf Operating oneer Netional Center

Nancy' A. Strow

Chitof Operating Omear

Field Cpersibons

Gortion L MaCullaugh

thicf Solence Officer

Acse Hacie Hobertson, MPD, FASTA

Erecutius tice frestident

teingen

Exeroúlive Vices Pressikent Curporate Opseratives a Eundar D. Joshi

Fseculive Vico Presidont

Conumusiowitions

Aubrym Lee Landrs, APR

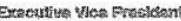
Corporets Swicretary \& Cansenal Crumsel

Davic Wm. Livingsesen, Ean.

Escrutva the Presklent

divariong

Miark A. Schoobarl

Ernersitiva Yica Prasildent

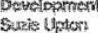

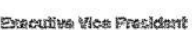

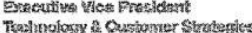
Thalus

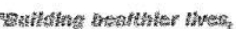

Thes at andthwersta?

ohoetstion and strote
American Heart | American Stroke

Association Association.

Learn and Live.

National Center

7272 Greenville Avenue $\bullet$ Dallas, Texas $75231-459$

Tel 214.373.6300 • americanheartorg

September 8, 2008

\section{ELECTRONIC AND PRINT COPYRIGHT USE AGREEMENT}

Becky Hainz-Baxter

McGraw-Hill

184 North Frances St.

Sunnyvale, CA 94086

Dear Ms. Hainz-Baxter:

Amount Due: $\mathbf{\$ 1 5 0 . 0 0}$ (WAIVED) (This is a fee for service, not a charitable contribution.) Our tax id number is 13-5613797. Please consider this letter an invoice.

Approval of this request is contingent upon receipt of a $\$ 150$ (waived) processing fee and a signed copy of this Agreement (including Exhibit A.) Please send a check payable to the American Heart Association with a copy of this Agreement to PO Box 841750, Dallas, Texas, 75284-1750.

The conditions of this copyright use agreement are listed below and the specifics of the material to be used are set out on Exhibit A to this Agreement.

1. A credit line must be prominently placed on the page in which the American Heart Association materials appear as shown in Exhibit A.

2. Original artwork cannot be supplied, and the requestor agrees to reprint the material exactly as originally published. The American Heart Association (AHA) logo and AHA Service Marks may only be used as they appear on the materials requested. Obvious typographical errors may be corrected and typography may be altered to conform to the proposed publication. No other deletions, alterations, or other changes may be made without the prior written consent.

3. American Heart Association materials are developed for educational nonprofit use only. We do not allow use of our materials to promote commercial products or companies or to imply an endorsement of, or affiliation with any particular organization.

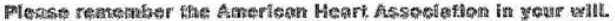


4. This permission is granted on a one-time use basis. Rights granted herein are not exclusive and do not apply to future reproductions, editions, revisions, or other derivative works including foreign language editions and do not apply to any versions via electronic media other than a password protected or subscription website site.

5. The website must require a subscription or password to access the material. Materials specified in Exhibit A must be opened and displayed on a separate page, containing only requested content, without trademark, or logos of the requesting party or any third parties. No additions, deletions or modifications may be made to this page. American Heart Association information must be posted as a "read only" document.

6. This permission is not valid until we receive payment, verifying email if paid electronically and a signed copy of this agreement. If the payment and the signed agreement are not received within 30 days, the request will be considered withdrawn and no longer in effect. No reminder notices will be sent.

Upon receipt of payment and the signed agreement, permission will be granted on your original request. Your cancelled check will be your receipt.

Signature of Requestor Sara Lifes

Printed Name Sara Leifer

Date $09108 / 08$ 


\title{
EXHIBIT A
}

Publication Name-American Heart Association-medmovie.com http://www.medmovie.com/mmdatabase/MediaPlayer.aspx?Client $I D=65 \&$ TopicID $=557$

Credit Line:

\author{
Reprinted with permission \\ American Heart Association/American Stroke Association \\ Figure: Coronary Artery Bypass Grafting (CABG) \\ (C)2004, medmovie.com
}

For Use In:

The CABG figure will be used in the introduction of Sara Leifer's Master Thesis in order to supplement the written portions with illustrations. The thesis is to be printed and a hard copy placed in the library at California Polytechnic State University - San Luis Obispo. Additional copies/pdf files will be sent to Sara Leifer's advisors via the Internet/Email. The thesis will not be published on the general Internet. 


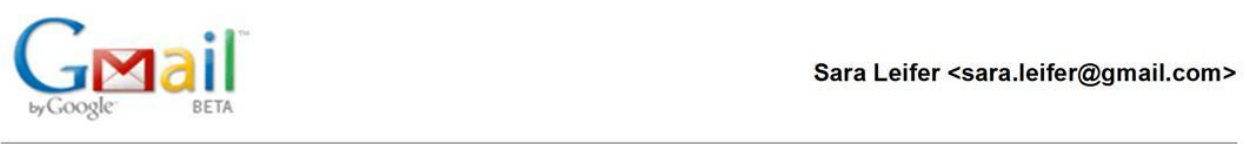

\section{Reprint Request CRM:00140835}

NHLBI Health Information Center <NHLBIInfo@nhlbi.nih.gov> To: sara.leifer@gmail.com

Mon, Sep 8, 2008 at 12:20 PM

Dear Ms. Leifer:

On behalf of the National Heart, Lung, and Blood Institute (NHLBI) Health Information Center, we are responding to your request for information on the NHLBI copyright policy.

The information contained in materials published by the NHLBI is in the public domain. No further permission is required to reproduce or reprint the information in whole or in part. However, organizations that reproduce NHLBI publications should cite the National Heart, Lung, and Blood Institute as a part of the National Institutes of Health and the U.S. Department of Health and Human Services as the source. This applies to printed publications as well as documents from the NHLBI Web site. Organizations may add their own logo or name. We further ask that no changes be made in the content of the material, and that the material as well as any NHLBI Internet links should not be used in any direct or indirect product endorsement or advertising.

Sincerely,

NHLBI Health Information Center

PO Box 30105

Bethesda, MD 20824

Phone: 301-592-8573

Fax: 301-592-8563

E-mail: nhlbiinfo@nhlbi.nih.gov

Web site: http://www.nhlbi.nih.gov

Achieve heart health goals easier than ever in 2009 with the vivid Keep the Beat wall calendar; discover creative healthy living tips and track heart disease risks with ease. Order your copies today at http://emall.nhlbihin.net/ktbcalendar. Customization and large quantity orders available. Call the NHLBI Health Information Center at 301-592-8573.

Have you let your voice be heard yet? The NHLBI would like to improve health information services for HIN members and is gathering feedback on the usefulness of current HIN information and preferences for future communications. If you have not already taken the brief 10-question survey, please go to either http://websurveyor.airws.org/ ss/wsb.dll/9/nhlbi_hin_3.htm or the NHLBI HIN Home page at http://hp2010.nhlbihin.net/ joinhin/index.asp, where the survey will pop up automatically. Thank you to all who have already responded!

http://mail.google.com/mail/?ui=2\&ik=6f25917662\&view=pt\&search=inbox\&msg=11c436 . 9/8/2008 